\title{
Development of a fiber optic sensor for hydrogen monitoring in transformers
}

by

Maximilian Fisser

\author{
A thesis \\ submitted to the Victoria University of Wellington \\ in fulfilment of the \\ requirements for the degree of \\ Doctor of Philosophy \\ in Engineering. \\ Victoria University of Wellington \\ 2018
}





\begin{abstract}
Electric vehicles and photovoltaic power generation are two of factors that are increasing the demands on the electrical grid. To cope with these challenges and to improve grid stability, the development of a transformer monitoring system as a fundamental part of the smart grid is necessary. Dissolved hydrogen in the transformer oil can serve as a primary indicator of the transformer health. Depending on the hydrogen concentration and rate of increase the transformer can be diagnosed.

The goal of this thesis was to develop a highly sensitive hydrogen sensor for online health monitoring of transformers.

The developed sensors are based on palladium and fiber Bragg gratings (FBG). Palladium expands with hydrogen absorption and this expansion is measured with an FBG. Detailed guidance for optimizing the sensor design is given. First, the selection of the working temperature is discussed. Second, the influence of the palladium geometry on the sensitivity is elaborated: by varying the cross-sectional area ratio of palladium to fiber the sensitivity can be tuned. Two different options to attach palladium are discussed: vapour deposition of palladium and adhesive bonding of palladium foils. The sensitivity of the palladium foil sensors was improved by improved manufacturing processes. The foil sensor have a sensitivity of up to $295 \mathrm{pm} / \%$ hydrogen and a resolution of $0.006 \%$ hydrogen in gas atmosphere at $90^{\circ} \mathrm{C}$ and $1060 \mathrm{mbar}$.

To further increase the hydrogen sensitivity two concepts for amplification of the signal are presented. One relies on palladium silver foils, which have an increased hydrogen solubility and therefore expansion compared to pure palladium, which achieved an increase in sensitivity of a factor of 17 . This leads to sensitivity of over $4500 \mathrm{pm} / \%$ hydrogen, which is the most sensitive hydrogen sensor reported so far. The other concept relies on a novel concept for strain concentration using a pre-strained palladium foil and FBG, which achieved an amplification of a factor of 2.5.

The sensors were characterised in gas and oil environment in a newly developed setup which is stable in pressure, temperature and gas concentration. In gas the sensors were tested at $60,75,90,105$, and $120^{\circ} \mathrm{C}$ and for a hydrogen concentration range of $0.01(100 \mathrm{ppm})$ to $5 \%$. In oil the sensor was tested at $90^{\circ} \mathrm{C}$ and for a hydrogen concentration range of 5- 4000 ppm dissolved hydro-
\end{abstract}


gen. Furthermore, the influence of carbon monoxide $(\mathrm{CO})$ on the hydrogen sensitivity was examined. A slowed response could be observed, but $\mathrm{CO}$ had no impact on the precision of the sensor.

Finally, the hydrogen calibration of the sensor is discussed by investigating the strain transfer between expanding palladium and fiber. Three different methods are elaborated to determine the coefficient of strain transfer: (a) via hydrogen measurement, (b) via temperature measurement, and (c) via strain measurement. Methods (a) and (b) were applied directly on the hydrogen sensor, and gave similar results. Method (c) was applied on a reference structure and used to verify method (b).

A hydrogen sensor suitable for transformer health monitoring has been developed and characterised, and is currently being implemented in a transformer in the New Zealand network. 


\section{Acknowledgements}

The last three years at the Robinson Research Institute have been a pleasant experience. Without doubt I would not have been able to finish this thesis in time without the help and support of the following people.

I was fortunate to have great supervisors with Paul Teal and Arvid Hunze, who guided me well through course of my PhD studies. Weekly meetings helped to structure my research from the beginning and to finish my thesis in time. I would not want to miss the valuable discussions and feedback I received, which helped me to grow as a scientist.

Without question, the biggest impact on my work has to be accredited to Rod Badcock. Giving me a profound introduction into fiber optic sensing, we had many discussion leading to new sensor designs and manufacturing processes. As a co-author of all my published work from this thesis, he influenced the quantity and quality of my publications.

I would like to thank the entire team at Robinson Research Institute. Special thanks goes to Mike Davies for continuous discussions, engineering assistance, and last but not least for sharing an office with me for most of the last three years. I also want to thank Gennady Sidorov for engineering assistance, Andres "Olly" Pantoja for help with LabView and measurements in general, Guy Dubuis for valuable discussion, Chris Bumby for the sharing his experience with strain measurements, Sarah Spencer for preparing the SEM images, and finally Jeannie Redman and Ursula Muavae for all the administrative work, that always frees up our time to do research.

I also want to thank the advanced materials group at Callaghan Innovation. Special thanks goes to Stefaan Janssens for teaching me about the handling of and measurements with fiber optic sensors. Furthermore, I want to thank Adam Swanson and Diego Del Puerto for setting up the second test chamber. I also want to acknowledge the workshop at Callaghan Innovation which manufactured several parts. Especially Robert Jakobsson, who at his time at Callaghan Innovation and afterwards at Llama Engineering, manufactured some high precision tools for us.

At this point, I want to thank the Ministry of Business, Innovation and 
Employment and ETEL Ltd. for funding the project. At ETEL especially Bhaba Das is to mention for his input and valuable discussions.

Finally, I want express my deepest gratitude towards my parents, family, friends, and especially my partner Bridget for creating the surroundings for a fast and successful completion of this thesis. 


\section{Contents}

1 Introduction 3

1.1 Motivation ..................... 3

1.2 Problem statement ...................... 3

1.3 Approach ....................... 5

1.4 Contribution of the thesis ............. 6

1.5 Structure of the thesis ............... 8

2 Literature review $\quad 11$

2.1 Dissolved gas analysis . . . . . . . . . . . . . . . . . 11

2.1.1 Transformer basics . . . . . . . . . . . . . . . 11

2.1.2 Faults in transformers and gas generation . . . . . . . . 12

2.1.3 Hydrogen as the main fault gas . . . . . . . . . . . 12

2.1.4 Hydrogen concentration and rate of increase . . . . . . 13

2.1 .5 DGA precision ................. 13

2.1.6 Online dissolved gas analysis and commercial solutions 14

2.2 The palladium hydrogen system . . . . . . . . . . . . . 14

2.2.1 Hydrogen in metals . . . . . . . . . . . . . . . 16

2.2.2 Hydrogen in palladium . . . . . . . . . . . 16

2.2.3 Hydrogen absorption . . . . . . . . . . . . 16

2.2.4 The phase diagram . . . . . . . . . . . . . 18

2.2.5 Hydrogen solubility in the $\alpha$-phase . . . . . . . . . 20

2.2.6 Changes in material properties . . . . . . . . . . 22

2.2 .7 Inhibiting gases . . . . . . . . . . . . . . . 23

2.3 Review of hydrogen sensors . . . . . . . . . . . . . . 23

2.3.1 Working principles . . . . . . . . . . . . . . 24

2.3.2 Fiber optic based hydrogen sensors . . . . . . . . . 25

2.4 The fiber Bragg grating . . . . . . . . . . . . . . . 26

2.4 .1 Application of FBG sensors . . . . . . . . . . . . . 29

3 Materials and Methods $\quad 31$

3.1 Material and Methods for Sensor Manufacturing . . . . . . . . . 31 
3.1.1 Fiber Bragg gratings . . . . . . . . . . . . . 31

3.1 .2 Fiber stripping $\ldots \ldots \ldots \ldots \ldots \ldots \ldots \ldots$

3.1 .3 Fiber etching .................... 32

3.1.4 Coating process - Sputter deposition . . . . . . . . 33

3.1 .5 Adhesives . . . . . . . . . . . . . . . 34

3.1.6 Palladium foils . . . . . . . . . . . . 35

3.2 Optical measurement equipment . . . . . . . . . . . . 35

3.2.1 Optical Spectrum Analyser - Anritsu MS9740A . . . . . . 36

3.2.2 FBG Interrogator - Micronoptics si155 . . . . . . . . . 36

3.2.3 Light Sources - Thorlabs S5FC1005S . . . . . . . . . . 36

3.2.4 Distributed feedback laser - Thorlabs S3FC1550 . . . . . 36

3.2.5 Fiber optic switch - DiCon GP700 . . . . . . . . . . . 36

3.3 Data processing .................... 36

3.3 .1 Peak detection . . . . . . . . . . . . . . . 37

3.3 .2 Compensation . . . . . . . . . . . 37

3.4 Test setups . . . . . . . . . . . . . . . . . . . . . 38

3.4.1 Setup I for measurements in gas . . . . . . . . . . 40

3.4.2 Setup II for measurements in gas and oil . . . . . . . . 43

3.4 .3 Gas chromatograph . . . . . . . . . . . . . 46

3.5 Tensile test . . . . . . . . . . . . . . . . . 46

3.5.1 Tensile test machine - Tinius Olsen H10KT . . . . . . . 47

3.5 .2 Tensile test bar . . . . . . . . . . . . . 47

4 Sensor design 49

4.1 Sensor parameter optimization . . . . . . . . . . . . . . 49

4.1.1 Selecting the working temperature to avoid phase change 49

4.1.2 Selecting the palladium geometry to achieve the required sensitivity .................... 52

4.1.3 Amplification of the signal to increase the sensitivity . . 53

4.2 Sensor design and manufacturing . . . . . . . . . . . . . . 55

4.2 .1 Coated sensor . . . . . . . . . . . . . . . 55

4.2.2 Wrapped $20 \mu \mathrm{m}$ palladium foil sensor . . . . . . . . 56

$4.2 .3100 \mu \mathrm{m}$ palladium foil sensor . . . . . . . . . . . . 58

4.2.4 $20 \mu \mathrm{m}$ sandwiched palladium foil sensors . . . . . . . . . 62

4.2.5 $10 \mu \mathrm{m}$ sandwiched palladium silver foil sensor . . . . . 63

4.2.6 Pre-strained $100 \mu \mathrm{m}$ palladium foil sensor . . . . . . 63

4.3 Sensor calibration . . . . . . . . . . . . . . 65 
5 Coated and first generation foil sensors $\quad 67$

5.1 Coated sensors . . . . . . . . . . . . . . 67

5.1 .1 Results ..................... 68

5.1 .2 Discussion . . . . . . . . . . . . . 69

5.2 Wrapped $20 \mu \mathrm{m}$ and $100 \mu$ mfoil sensors . . . . . . . . . 71

5.2 .1 Results .................... 72

5.2 .2 Discussion . . . . . . . . . . . . . 74

5.3 Comparison of the performance . . . . . . . . . . 75

5.4 Conclusion . . . . . . . . . . . . . . . . 77

$6 \quad$ Second generation foil sensors $\quad 79$

6.1 Wrapped $20 \mu \mathrm{m}$ palladium foil sensor . . . . . . . . . . 80

6.1 .1 Results .................. 80

6.1 .2 Discussion .................. 80

$6.2100 \mu$ m palladium foil sensor . . . . . . . . . . . 82

6.2 .1 Results .................... 83

6.2 .2 Discussion ................... 83

6.3 Sandwiched $20 \mu \mathrm{m}$ palladium foil sensor . . . . . . . . . 84

6.3 .1 Results ................... 84

6.3 .2 Discussion . . . . . . . . . . . . . 85

6.4 Comparison of the performance . . . . . . . . . . . . . 87

6.5 Summary and conclusion . . . . . . . . . . . . . 88

7 Amplification concepts $\quad 89$

7.1 Sandwiched $10 \mu \mathrm{m}$ palladium silver foil . . . . . . . . . . 89

7.1 .1 Results . . . . . . . . . . . . . . . 90

7.1 .2 Discussion . . . . . . . . . . . . . . 90

7.2 Pre-strained sensor . . . . . . . . . . . . . . . . 92

7.2 .1 Results . . . . . . . . . . . . . . 92

7.2 .2 Discussion ..................... 93

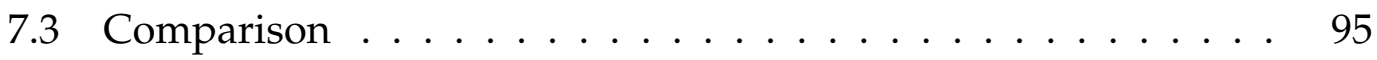

7.4 Summary and conclusion . . . . . . . . . . . . 97

8 Characterisation in gas and oil $\quad 99$

8.1 Characterisation in gas . . . . . . . . . . . . . . . . 99

8.1 .1 Response time . . . . . . . . . . . . . 101

8.1.2 Carbon monoxide cross-sensitivity . . . . . . . . . . . . 101

8.1 .3 Discussion . . . . . . . . . . . . . 105

8.2 Characterisation in oil . . . . . . . . . . . . . . 108

8.2 .1 Discussion ................... 112 
8.3 Conclusion . . . . . . . . . . . . . . . . . 113

9 Coefficient of strain transfer and sensor calibration $\mathbf{1 1 5}$

9.1 Determined via a hydrogen measurement . . . . . . . . . . . 115

9.1.1 Influence on the sensor calibration . . . . . . . . . . . 116

9.2 Determined via a temperature measurement . . . . . . . . . . 121

9.2.1 Influence on the sensor calibration . . . . . . . . . . . 123

9.3 Determined via a strain measurement . . . . . . . . . . . . 126

9.3.1 Experimental method . . . . . . . . . . . . . 127

9.3 .2 Results . . . . . . . . . . . . . . . 128

9.4 Summary and conclusion . . . . . . . . . . . . . 131

$\begin{array}{ll}10 \text { Summary and future work } & 133\end{array}$ 


\section{Abbreviations}

CSA............ Cross-sectional area

DFB............ Distributed feedback laser

DGA............ Dissolved gas analysis

FBG............. Fiber Bragg grating

H.................. Hydrogen

K................. Sieverts' constant laser

KOH............ Potassium hydroxide

n................. Ratio of hydrogen to palladium atoms

Ormocer..... Organically modified ceramic

OFS............ Optical fiber sensor

OSA........... Optical spectrum analyser

Pd............... Palladium

PdAg........... Palladium-silver-alloy

PTFE........... Polytetrafluoroethylene

SEM........... Scanning electron microscope

SLD............ Super luminescent diode

$\mu \varepsilon$................ Microstrain, $10^{-6} \Delta l / l$

$\lambda$................. Center wavelength of FBG peak 


\section{Chapter 1}

\section{Introduction}

Active asset management is of increasing importance for electrical grid companies because of growing demands of users. e.g., more photovoltaic and electric vehicles [56]. Some of the main - and most costly - components of the grid are distribution and power transformers. The health status of a transformer can be assessed by dissolved gas analysis (DGA) [68]. Gases are generated in the transformer during faults and subsequently dissolve into the oil. Hence, dissolved gas concentration can be correlated to the transformer health status. Conventionally, oil samples are taken at given time intervals e.g., annually and analysed in a laboratory for their dissolved gas concentration. A more recent approach is to monitor the gases online. DGA is mainly performed on large and expensive power transformers, and rarely on less expensive and smaller distribution transformers [106].

\subsection{Motivation}

Several manufacturers provide devices for online DGA. However, due to their cost, those devices are exclusively installed on larger, expensive power transformers. Inexpensive solutions which could satisfy the market as a monitoring systems for smaller and less costly distribution transformer are not available commercially. In a project with our industry partner ETEL Limited, Auckland we focussed on the development of a cost effective, fiber optic hydrogen sensor for the health monitoring of transformers.

\subsection{Problem statement}

Hydrogen is the most significant fault gas in a transformer and is generated during both normal operation, and especially during thermal and electrical 


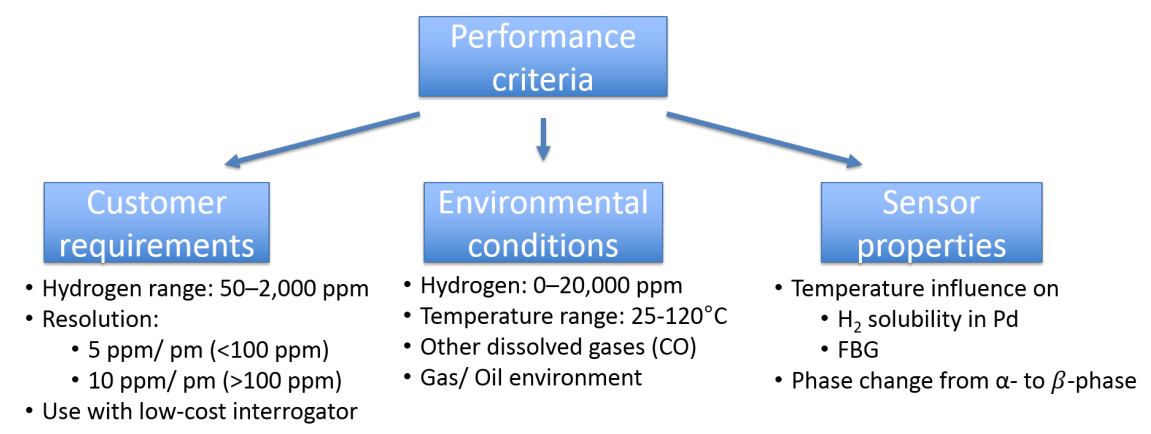

Figure 1.1: Performance criteria of the research and development process

faults (e.g., partial discharge, overheating) and thus can serve as a primary health indicator [68].

Different concepts of hydrogen sensors have been reported [65]. Optical fiber sensor (OFS) have the advantage of being intrinsically safe in combustible gases, not breaking the electrical insulation and being insensitive to electromagnetic noise [65], all aspects which are crucial for an implementation in a transformer. Palladium is often used as a sensing element. Palladium dissociates the molecular hydrogen on its surface, before atomic hydrogen is absorbed in the bulk. The absorption leads then to the expansion of the lattice [82]. One concept of OFS relies on measuring this expansion of palladium with a fiber Bragg grating (FBG).

Recent reports of some palladium based FBG hydrogen sensors for DGA use thin palladium coatings to deliver a fast response [71,91] . However, DGA usually does not require fast response time due to the relatively slow production of hydrogen in the transformer. A response time of hours or even days would be acceptable as standard sampling intervals are rarely shorter than weekly [49]. More important is an accurate reading of the hydrogen concentration and the rate of increase to determine the health status of a transformer [68]. Therefore, for this application the sensitivity of the sensor is our main optimization parameter. A highly sensitive sensor (large wavelength changes), would also allow the use of a low-cost FBG interrogator with a lower wavelength stability compared to expensive, high-end laboratory equipment.

Early on in the project, performance criteria were defined to help guide through the sensor development process. Three classes of performance criteria were identified (see Figure 1.1): customer requirements, environmental conditions, and sensor properties.

The customer requirements were defined and agreed with ETEL Limited: Following the IEEE [68] and IEC [67] standard a dissolved hydrogen sensing range of $50-2000 \mathrm{ppm}$ is desirable. The resolution of the sensor should be 
$5 \mathrm{ppm} / \mathrm{pm}$ for concentrations below $100 \mathrm{ppm}$ hydrogen and $10 \mathrm{ppm} / \mathrm{pm}$ for concentrations above. This resolution would allow the use of a low-cost interrogator.

The environmental conditions are give by the transformer environment: The hydrogen concentration in small distribution transformers is reported to be as high as 20,000 ppm [106], the temperature can range from $25-120^{\circ} \mathrm{C}$ and other dissolved gases (e.g., carbon monoxide) are present. The developed sensor should withstand these conditions. Additionally, the sensor should be able to measure dissolved hydrogen in oil, and gaseous hydrogen in the transformer head-space.

Palladium and FBG, both have characteristic properties which can impact the sensor's hydrogen sensitivity, temperature cross-sensitivity, and durability. Temperature influences the hydrogen solubility in palladium and thus the expansion. Additionally, palladium undergoes a phase change (depending on temperature and hydrogen partial pressure) which has to be avoided to guarantee sensor stability. Finally, FBGs are not only excellent strain sensors but also highly sensitive to temperature changes.

In conclusion, the sensor requirements and specification can be summarised as follows: the sensor should be highly sensitive in gas and oil in a range from $50-2,000$ ppm dissolved hydrogen and stable in concentrations up to 20,000 ppm, it should be selective and have a low cross-sensitivity to other gases and temperature. Of course, the sensor should be fully reversible in response, durable, and cheap [115].

\subsection{Approach}

The approach for the sensor development was as follows. First, different sensor designs were developed and tested in gas. Then, the design with the best performance was selected and fully characterised (using several hydrogen concentrations and at different temperatures) in gas and oil environment. The main test conditions were defined together with our industry partner ETEL: $90^{\circ} \mathrm{C}$, which is over the average working temperature of a transformer and just below the temperature of increased oil degradation [102], and 1060 mbar which can be assumed to be the average pressure in a transformer.

Initially, a conventional approach was followed for the sensor design: coating a fiber with palladium. An increase in sensitivity was attempted by decreasing the fiber diameter with etching (down to $85 \mu \mathrm{m}$ ) and increasing the coating thickness (up to $1600 \mathrm{~nm}$ ). However, the desired sensitivity was not reached. These results were consistent with our theoretical model. Our cal- 
culation predicted that a coating thickness of tens of micrometers would be required to achieve the desired sensitivity. Since the coating process for such thickness would take days and the outcome (layer stability, delamination) was questionable, alternative solutions were needed. These were found in palladium foils that are adhesively bonded to a fiber. After initial testing and proofing of the principle, several sensor concepts with different foil thickness were designed and corresponding manufacturing processes were developed. The most suitable sensor for the integration in a transformer proved to be based on a $100 \mu \mathrm{m}$ palladium foil, which was fully characterised in both gas and oil environments.

Over the course of this thesis different factors that influence the hydrogen sensitivity were identified. These factors are shown in a cause and effect diagram in Figure 1.2 and can be categorised as follows. The sensing Environment influences the sensitivity via the hydrogen partial pressure and the temperature, but also via whether the hydrogen is dissolved in a liquid, or in gas (see Sections 2.1.5, 2.2.4, 2.2.5, and 4.1.1). The palladium Geometry affects the sensitivity via the cross-sectional area ratio of palladium to fiber, and possible strain concentration variation (see Section 4.1.2, and 4.1.3). The Transducer (strain sensor) inherently limits the hydrogen sensitivity via its strain sensitivity (see Section 2.4). The sensor Material (Palladium, or palladium alloy) affects the sensitivity via its volumetric expansion, which depends on the hydrogen solubility and thus on alloying materials (see Sections 2.2.5, and 4.1.3). The Strain transfer between expanding palladium and fiber depends on the adhesion between the palladium, fiber, the adhesive (mechanical) properties and the bond line thickness (see Section 4.2, and 4.3). The Signal to Noise ratio influences the sensitivity directly via the temperature cross-sensitivity of the sensor and the wavelength stability of the interrogating equipment (see Section 3.3.2).

\subsection{Contribution of the thesis}

The research contribution of this work can be divided into three categories: sensor parameter optimization, sensor design and manufacturing, and sensor calibration.

The sensor parameter optimization include the selection of the working temperature of the sensor to avoid the phase change region, and the selection of the palladium geometry to achieve a desired sensitivity.

The sensor design and manufacturing include the sensor designs that have a palladium foil attached to the fiber, a manufacturing process for wrapping 


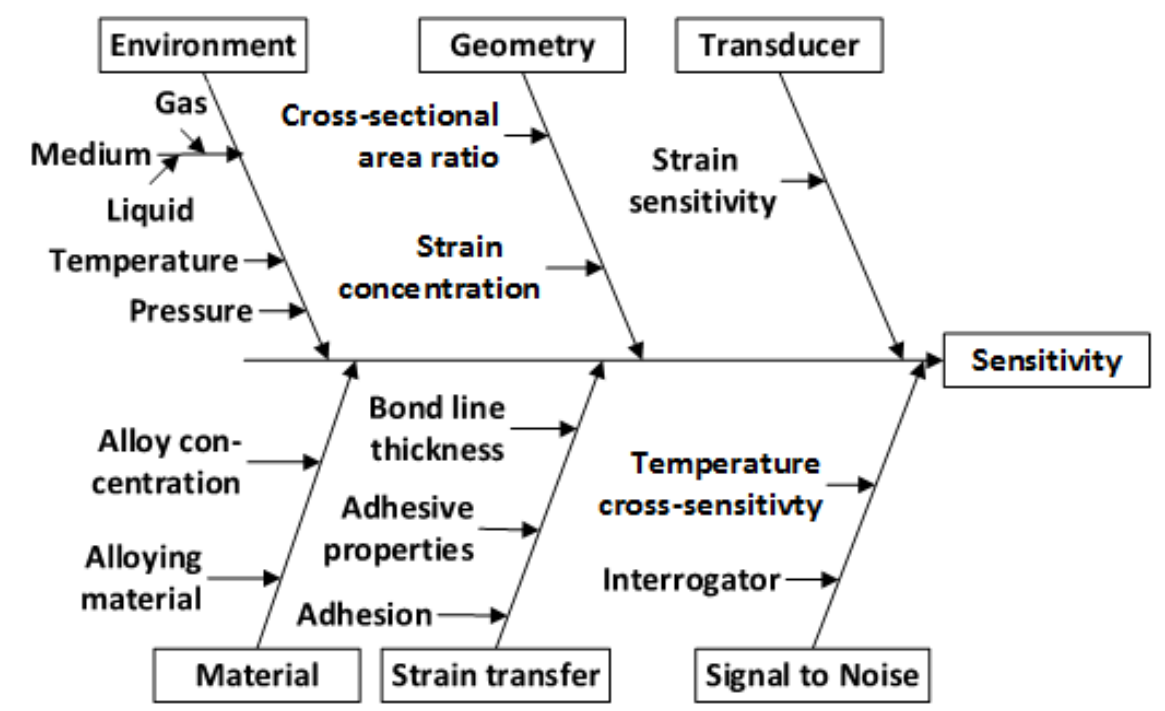

Figure 1.2: Cause and effect diagram of the factors that influence the hydrogen sensitivity of palladium based sensors.

palladium foil around a fiber, and using vacuum bagging as a method to attach a foil to a fiber. Furthermore, a novel sensor concept with a pre-strained FBG and palladium foil is presented.

The sensor calibration includes the calibration of the sensor via a coefficient of strain transfer. This coefficient can be determined via three methods: hydrogen measurement, temperature measurement and strain measurement. The findings allow the hydrogen calibration of the sensors via a single hydrogen measurement, or via a temperature sensitivity calibration. In addition, a concept for in situ strain calibration of surface bonded FBGs via temperature calibration is shown.

During the course of this thesis several publications have been prepared: one patent application, three accepted conference papers, and three accepted journal papers (two further journal papers are in preparation). A list of the conference and journal publication is given below. Several passages and figures, that were published, or are currently in preparation for publication have been directly reused or were reproduced for this thesis.

The journal papers are:

1. Maximilian Fisser, Rodney A. Badcock, Paul D. Teal, Stefaan Janssens, Arvid Hunze, "Palladium based hydrogen sensors using fiber Bragg gratings", Journal of Lightwave Technology, 2017.

DOI: $10.1109 /$ JLT.2017.2713038

2. Maximilian Fisser, Rodney A. Badcock, Paul D. Teal, Arvid Hunze, "Optimizing the sensitivity of palladium based hydrogen sensors", Sensors 
and Actuators B: Chemical 259, 2018, 10-19.

DOI: $10.1016 /$ j.snb.2017.11.180

3. Maximilian Fisser, Rodney A. Badcock, Paul D. Teal, Arvid Hunze, "Improving the sensitivity of palladium based fiber optic hydrogen sensors", Journal of Lightwave Technology, 2018. (Accepted for publication)

4. Maximilian Fisser, Rodney A. Badcock, Chris W Bumby, Paul D. Teal, Arvid Hunze, "In situ strain calibration of fiberoptic sensors via temperature calibration", Journal of Lightwave Technology, 2018. (In preparation)

5. Maximilian Fisser, Rodney A. Badcock, Paul D. Teal, Arvid Hunze, "Highly sensitive fiber optic hydrogen sensor for health monitoring of transformers", Sensors and Actuators B: Chemical, 2018. (In preparation)

The conference papers are:

1. Maximilian Fisser, Rodney A. Badcock, Paul D. Teal, Arvid Hunze, "Palladium based hydrogen sensors using fiber Bragg gratings", Proc. SPIE 10323, 25th International Conference on Optical Fiber Sensors, 103231X, 2017. DOI: $10.1117 / 12.2262383$

2. Maximilian Fisser, Rodney A. Badcock, Paul D. Teal, Adam Swanson, Arvid Hunze, "Development of hydrogen sensors based on fiber Bragg grating with a palladium foil for online dissolved gas analysis in transformers", Proc. SPIE 10329, Optical Measurement Systems for Industrial Inspection X, 103292P, 2017. DOI: 10.1117/12.2267091

3. Maximilian Fisser, Rodney A. Badcock, Paul D. Teal, Arvid Hunze, "Optimizing the sensitivity of palladium based fiber optic hydrogen sensors", 3rd Australian and New Zealand Conference on optics and photonics, 2017. (Proceedings not published yet)

The patent application was filed on the 30th of September 2016, but was not published before submission. It can be found under New Zealand patent number 734975.

\subsection{Structure of the thesis}

The thesis is organized in ten chapters as follows: 
Chapter 2 gives a literature review and presents background knowledge: Existing literature regarding hydrogen sensors in transformers, the fundamentals of the palladium hydrogen system, different concepts of hydrogen sensors, and the basics of fiber Bragg gratings are presented. This enables the reader to better understand the work in the context of the current literature.

Chapter 3 introduces the materials and methods used in this thesis. All relevant materials and processes that were used to manufacture the sensors are described. The equipment to interrogate the sensors is presented and the data processing approach is explained. Finally, the test setups are described.

Chapter 4 discusses the sensor design. First, the sensor optimization parameters are discussed: the selection of the working temperature to avoid phase change, the selection of the palladium geometry to achieve a desired sensitivity, and concepts to amplify the signal to increase the sensitivity. Second, the sensor design and manufacturing of thin film (coated) sensors, newly developed foil sensors, and a new sensor concept with a pre-strained FBG are described. Finally, the calibration of the sensor via the coefficient of strain transfer is explained.

Chapter 5 presents the results of a coated sensor and the newly developed foils sensors. The sensor designs are compared in terms of response time and sensitivity. Furthermore, the theoretical and actual sensitivity are compared.

Chapter 6 presents the results of the second generation of foil sensors. The results show that the improved manufacturing methods increase the sensitivity of the sensors.

Chapter 7 presents the results of sensor concepts that amplify the signal to increase the hydrogen sensitivity. The evaluated sensors concepts are a palladium silver foil sensor and an original sensor concept using a pre-strained FBG and palladium foil.

Chapter 8 presents the results of the characterisation of the $100 \mu \mathrm{m}$ palladium foil sensor. The sensor is characterised in hydrogen mixtures of 0 to $5 \%$ and temperatures from $60-120^{\circ} \mathrm{C}$. Furthermore, the cross-sensitivity to carbon monoxide is analysed. Finally the sensor is characterised in transformer oil at $90^{\circ} \mathrm{C}$ for dissolved hydrogen concentrations from 0 to around $4500 \mathrm{ppm}$.

Chapter 9 investigates the coefficient of strain transfer and its influence on the hydrogen calibration of the sensor. Three different ways to determine the coefficient are given: via hydrogen, via temperature, and via strain measurement. Furthermore, the influence of the coefficient of strain transfer on the accuracy is discussed.

Chapter 10 summarizes the work presented in this thesis, draws a conclusion and discusses potential, future work. 


\section{Chapter 2}

\section{Literature review}

In this chapter, the necessary background knowledge is explained. Firstly, dissolved gas analysis is elaborated, by discussing voltage transformers, faults that occur during their use, gases that are generated as a consequence of these faults, and how they dissolve into the oil. Secondly, the palladium hydrogen system is elaborated, by discussing hydrogen in metals in general, hydrogen absorption in palladium, the resulting material property changes, and gases that inhibit the hydrogen absorption. Thirdly, different concepts of hydrogen sensors are discussed. Finally, fiber Bragg gratings (FBG) are elaborated.

\subsection{Dissolved gas analysis}

Dissolved gas analysis (DGA) is a method that electrical grid companies have used to maintain transformers and determine their health status since the 1970s [46]. A fitting analogy is given by Duval [46] who compares DGA to a blood test: an oil (blood sample) is taken and sent of to a laboratory. With the returning results the operator (doctor) can determine the health status of the transformer (patient) and initiate maintenance (treatment). DGA can be beneficial because, if a fault is detected early, the transformer can be examined and possible future faults and failure may be prevented [66].

This section covers the transformer basics, faults that occur in a transformer, and the generation of gases due to these faults.

\subsubsection{Transformer basics}

Transformers are an essential part of the electric grid; transforming the voltage up or down for low loss transmission (power transformer) and transforming the voltage down for distribution (distribution transformer) to the end-user. The two transformer types also have different power ratings. Distribution 
transformer are in the range of $50-1600 \mathrm{kVA}$ whereas power transformers have a rating greater 2 MVA [57]. Transformers change the voltage by generating a magnetic field with a coil that subsequently induces a voltage in another coil. Thus, the coils need to be electrically insulated. Conventionally the insulation is achieved by mineral oil and paper [63]. The oil also serves the purpose of a cooling liquid transferring the heat generated by the coils through convection. Conditional on usage, power transformers operate under higher loads, are larger in size, and have a larger oil volume, than distribution transformers.

\subsubsection{Faults in transformers and gas generation}

Faults frequently occur in transformers. A fault is an unplanned, undesirable event that finally could cause failure. These faults can be categorized as electrical faults (partial discharge, arcing) and thermal faults (excessive temperature rise) [66].

Faults cause decomposition of the oil. Mineral oil is composed of different hydrocarbon molecules, and their break down causes the formation of gases [66]. The type of gases formed depends on the fault. Low-energy faults (e.g., partial discharge) mainly cause the formation of hydrogen by breaking down the carbon-hydrogen bond. High energy faults (e.g. arcing) cause the formation of new hydrocarbons (e.g., methane, ethylene, acetylene, and ethane) by breaking down the carbon-carbon bond. Furthermore, most high energy faults also generate hydrogen [47].

Faults can also cause the decomposition of the insulating paper [66]. The gases formed due to the decomposition of the paper are mostly carbon monoxide and carbon dioxide. $[66,68]$.

\subsubsection{Hydrogen as the main fault gas}

Of all the generated and initially present gases, nine are analysed in a DGA [66, 68]: Hydrogen, methane, acetylene, ethylene, ethane, carbon monoxide, carbon dioxide, nitrogen, and oxygen.

Hydrogen can serve as primary indicator of the overall transformer health, since it is formed during most faults rather than being limited to a specific fault. In addition, usually low energy faults, which generate mainly hydrogen, exist over a period of time before higher energy faults occur, which can possibly lead to failure of the transformer [66]. 


\subsubsection{Hydrogen concentration and rate of increase}

DGA values are given in $\mu \mathrm{L} / \mathrm{L}$ or ppm of dissolved gas. The status of a power transformer can be divided into 4 conditions depending on the hydrogen concentration. Normal hydrogen concentration in a power transformer is around $100 \mathrm{ppm}$ and failure can be expected for values higher than $1800 \mathrm{ppm}[66,68]$.

Apart from the absolute hydrogen concentration the rate of increase is of importance, since the rate can give an indicaton of whether the fault has stoped, or is still occuring, or even growing [66]. In power transformers normal rates of increase are $88 \mathrm{ppm} /$ year and can go up to $725 \mathrm{ppm} /$ year just before failure [49].

The afformentioned values are for large power transformers. In smaller distribution transformers a higher concentration can be expected due to a smaller oil volume [68] (see Section 2.1.1). Though not many studies regarding the dissolved gas concentration in distribution transformers can be found, a large scale investigation of DGA values of distribution transformers in the USA showed that hydrogen levels up to 2000 ppm are normal in distribution transformer with levels reaching up to 27000 ppm before failure [106].

The hydrogen concentration and rate of increase also determines how often a DGA is performed. Suggested sampling intervals are in the range of annual for a normally operating power transformer (low concentration and rate of increase) to daily for abnormally operating (very high concentration and rate of increase) [68]. Only for abnormally operating power transformers in the transmission grid or at nuclear power plants are hourly sampling intervals suggested [49].

\subsubsection{DGA precision}

The precision of DGA measurements can be critical and the uncertainty of the results can be high. This is because, firstly, there are different methods to sample and to extract the gases from the oil $[48,67]$. Secondly, the time between sampling and analysing can have an impact on the accuracy, as gases can diffuse out of the oil. Thirdly, the accuracy of the gas chromatograph has an influence. Fourthly, the measured concentration in the gas phase is correlated to the dissolved gas concentration in the liquid phase via the Ostwald coefficient $k_{\text {Ost. }}$ which depends on the temperature and type of oil [66].

$$
k_{\text {Ost. }}=\frac{\text { concentration of gas in liquid phase }}{\text { concentration of gas in gas phase }}
$$

Ostwald coefficients for different temperatures and different oils found in 
the literature are 0.043 [68], 0.05 [100], and 0.056 [66] at $25^{\circ} \mathrm{C}$ and $0.92-0.97$ at $70^{\circ} \mathrm{C}[100]$.

All these factors increase the uncertainty of the measurement and lead to a precision of $5 \%$ for DGA values larger than 10 times the analytical detection limit and $20 \%$ for values below that [67]. Lelekakis et al. compared five laboratories and found deviation of up to $45 \%$ for an oil sample with $10 \mathrm{ppm}$ dissolved hydrogen [81] .

\subsubsection{Online dissolved gas analysis and commercial solutions}

Online DGA devices are directly connected to the transformer and measure the dissolved gases continuously. Several commercial solutions are available (see Table 2.1). All systems analyse hydrogen, with some analysing all nine fault gases. For hydrogen detection either gas chromatography, photo acoustic spectrocopy, resistance based sensors, thermal conductivity detectors, or electro chemical sensors are used. [31,50]

The maximum range of a sensor for hydrogen is 0 to $50000 \mathrm{ppm}$ with most sensors in the range of 20 to 5000 ppm (compare Table 2.1). The gas chromatograph has the highest accuracy with 2 ppm, or 5\% (whichever is higher). Most sensors have an accuracy of 25 ppm, or $20 \%$ (whichever is higher)

The sampling interval of the gas chromatograph type monitors is between 1 and 12 hours [101,122] with a 95\% response to a step increase after 1 hour [101].

The response time for the resistance based sensors is faster than 60 minutes [123] to 17 hours [136] for a $90 \%$ response to a step increase. The thermal conductivity detector is advertised as significantly faster with 10 minutes for a $90 \%$ response [53].

The cost of the listed systems is high, and thus they are mainly used for power transformers. A cost-effective solution for distribution transformers is desirable and its development is the aim of this thesis.

\subsection{The palladium hydrogen system}

Hydrogen, the smallest atom, is absorbed in several metals. The most reactive metal, however, is Palladium. Palladium not only serves as a catalyst and dissociates molecular hydrogen into atomic hydrogen, but also absorbs large quantities of the atomic hydrogen. The absorbance of hydrogen in palladium was first reported by Graham in 1869 [60]. A first review of the palladiumhydrogen system was given by Lewis in 1967 [82]. A more extensive summary of metal-hydrogen systems, including palladium and its alloys was given by 
Table 2.1: Commercial online DGA solutions. Sensor type: Gas chromatograph (GC), photo acoustic spectroscopy (PAS), resistance based sensor (RS), thermal conductivity detector (TCD), or electro chemical sensor (ECS). Range in ppm accuracy in ppm/\% (whichever is higher).

\begin{tabular}{llrr}
\hline Model & Sensor type & Range (ppm) & Acc.(ppm/ \%) \\
\hline Calisto 5 / Calisto 9 [50,101] & GC & $0.5-20000$ & $-/ 5$ \\
SERVERON TM8 [121] & GC & $3-3000$ & $3 / 5$ \\
Siemens GAS-Guard [122] & GC & $2-3000$ & $2 / 5$ \\
GE kelman minitrans [50,55] & PAS & $5-5000$ & $5 / 10$ \\
ABB coresense [1,2] & SSS (Pd alloy) & $25-5000$ & $25 / 20$ \\
Calisto, Calisto 2 [31,50,101] & TCD, membrane & $2-50000$ & $5 / 5$ \\
GE Hydran M2 [54] & ECS, membrane & $0-2000$ & $25 / 10$ \\
GE Hydran 201Ti [53] & ECS, membrane & $0-2000$ & $25 / 10$ \\
QUALITROL DGA 150 [50,113] & RS (Pd/Ni) & $50-5000$ & $25 / 20$ \\
SERVERON TM1 [50,120] & RS (Pd/Ni) & $20-10000$ & $20 / 15$ \\
Siemens Sitram H2Guard [123] & RS (Pd/Ni) & $25-5000$ & $25 / 20$ \\
Vaisala MHT410 [136] & RS (Pd/Ni) & $20-5000$ & $25 / 20$ \\
Weidmann InsuLogix H [142] & RS (Pd/Ni) & $20-5000$ & $25 / 20$ \\
\hline
\end{tabular}


Alefeld et al. in 1978 [6,7]. In this section we explain the effect of hydrogen absorption in metals and in detail in palladium, the material property changes that come along with the absorption, and the effect other gases have on the hydrogen absorption.

\subsubsection{Hydrogen in metals}

Hydrogen is absorbed in several metals (e.g.: iron, steel, niobium, tantalum, vanadium, nickel, and palladium and several of their alloys [6]). The absorptions always causes an expansion of the lattice. Palladium is often chosen as a sensing material, because, unlike to most other metals, the palladium hydride is formed at ambient conditions (low pressure and temperature) [3].

\subsubsection{Hydrogen in palladium}

Molecular hydrogen is dissociated into its atoms on the palladium surface, before diffusing into the palladium lattice. There, the hydrogen atoms randomly occupy octahedral sites, until an equilibrium with the surrounding medium is reached. The amount of hydrogen in the palladium lattice at equilibrium is described with the ratio $n$ of dissolved hydrogen atoms to palladium atoms. $n$ increases with increasing pressure (hydrogen partial pressure) and decreasing temperature. [7]

\subsubsection{Hydrogen absorption}

The absorption can be divided into three sub processes: the adsorption of molecular hydrogen on the palladium surface (2.2), the dissociation of adsorbed, molecular hydrogen into atoms (2.3), and the absorption of atomic hydrogen into the palladium lattice (2.4) [7].

$$
\begin{gathered}
\mathrm{H}_{2, \text { gas }} \rightleftharpoons \mathrm{H}_{2, \mathrm{ad}} \\
\mathrm{H}_{2, \mathrm{ad}} \rightleftharpoons 2 \mathrm{H}_{\mathrm{ad}} \\
\mathrm{H}_{\mathrm{ad}} \rightleftharpoons \mathrm{H}_{\mathrm{ab}}
\end{gathered}
$$

Of the 3 steps, step 2 ,the dissociation of adsorbed, molecular hydrogen is the rate determining step of the absorption [7]. 
The diffusion rate by itself can be calculated with the differential diffusion equation:

$$
\frac{d n}{d t}=D \frac{d^{2} n}{d x^{2}}
$$

where $n=\mathrm{H} / \mathrm{Pd}$ (mole ratio), $D$ is the diffusion coefficient $\left(\mathrm{m}^{2} / \mathrm{s}\right)$ and $x$ is the location in the palladium relative to the surface. The diffusion coefficient follows the Arrhenius behaviour and can be calculated as $[6,20,85]$

$$
D=D_{0} e^{-\frac{E_{\mathrm{a}}}{R T}}
$$

where $D_{0}$ is the diffusion coefficient of hydrogen in $\mathrm{m}^{2} / \mathrm{s}, E_{\mathrm{a}}$ is the activation Energy $\mathrm{J} / \mathrm{mol}, \mathrm{R}$ is the general gas constant in $\mathrm{J} / \mathrm{molK}$ and $\mathrm{T}$ is the temperature in K. Reported values for $D_{0}$ range from $3.9 \times 10^{-9} \mathrm{~m}^{2} / \mathrm{s}$ for thin films [85] to $5.7 \times 10^{-7} \mathrm{~m}^{2} / \mathrm{s}$ for bulk material [23]. Reported values for $E_{\mathrm{a}}$ range from $37.4 \mathrm{~kJ} / \mathrm{mol}$ for thin films to $22.2 \mathrm{~kJ} / \mathrm{mol}$ for bulk material [85]. Alefeld et al. compared 25 different studies and found $2.9 \times 10^{-7} \mathrm{~m}^{2} / \mathrm{s}$ for $D_{0}$ and $22.2 \mathrm{~J} / \mathrm{mol}$ for $E_{\mathrm{a}}$ to be the best fit [6].

Equation 2.5 can be solved with the initial condition $n=0$, Dirichlet boundary condition $c=1$ (constant hydrogen concentration on the palladium surface at all times) and separation of variables to obtain [112]:

$$
n(x, t)=c_{o}\left(1-\frac{2}{\pi} \sum_{z=1}^{\infty} \frac{1-(-1)^{z}}{z} \sin \left(\frac{z \pi x}{\delta}\right) \exp \left(-\mathrm{D}\left(\frac{z \pi}{\delta}\right)^{2} t\right)\right)
$$

where $\delta$ is the thickness of the sample, $x$ is the position and $t$ is the time in seconds.

Using equation (2.6) the hydrogen diffusion in a $100 \mu \mathrm{m}$ palladium foil is simulated and shown in Figure 2.1. Hydrogen is fully diffused into the center $(50 \mu \mathrm{m})$ of the foil in less than 30 seconds.

However, as mentioned, the dissociation and not the diffusion is the rate determining step of the hydrogen absorption. The absorption rate (taking the dissociation as the rate determining step into account) for small $n$ - where Sieverts's law is applicable - can be calculated according to Auer and Grabke [13]:

$$
\nu=\frac{\delta}{2 \cdot V_{\mathrm{Pd}}} \cdot \frac{d n}{d t}
$$

where $\nu$ is the reaction rate $\left(\mathrm{molH} / \mathrm{cm}^{2} \mathrm{sec}\right), \delta$ is the thickness of the sample and $V_{\mathrm{Pd}}$ is the molar volume. $\nu$ is given by

$$
\nu=k \cdot p_{H 2} \frac{1}{1+K \cdot n}-k^{\prime} \frac{K \cdot n^{2}}{1+K \cdot n}
$$




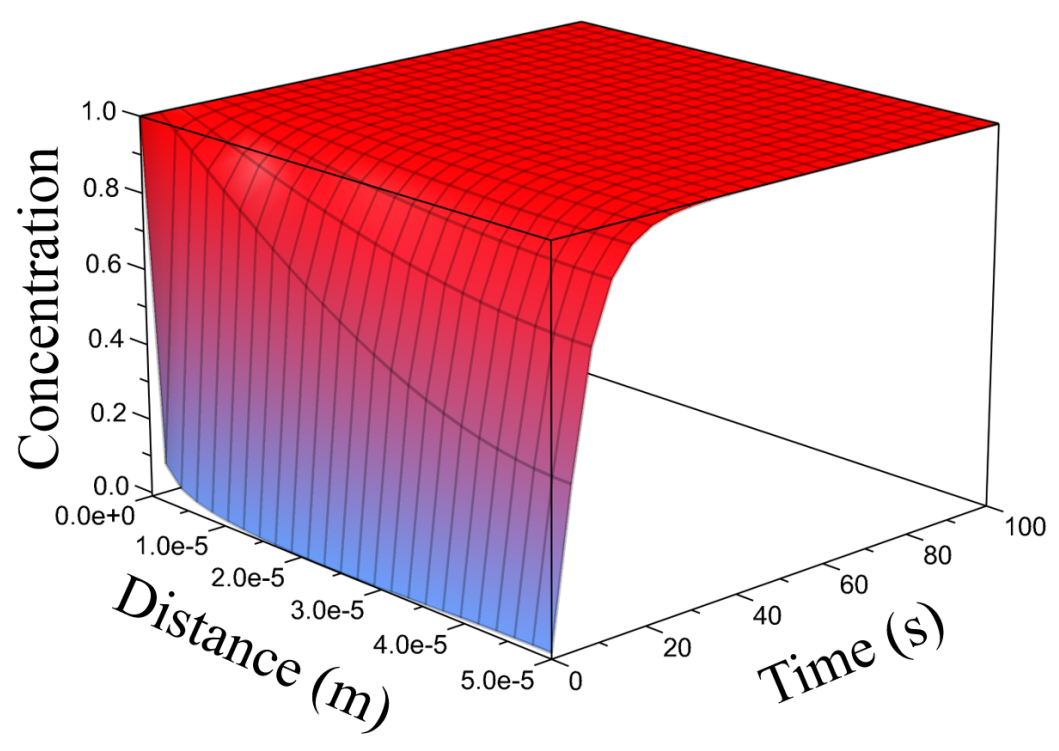

Figure 2.1: Solution to the diffusion equation for a $100 \mu \mathrm{m}$ palladium foil with $\mathrm{D}=1.85 \times 10^{-10} \mathrm{~m}^{2} / \mathrm{s}$ at $90^{\circ} \mathrm{C}$.

where $K$ is Sieverts's constant and $k$ and $k^{\prime}$ are the rate constants for absorption and desorption. The first term is representative for the absorption process and the second term for the desorption process. When both terms are equal the palladium hydride is in a state of equilibrium.

The solution of equation (2.8) is the absorption time given by:

$$
t=\frac{\delta}{2 \cdot V_{\mathrm{Pd}}}\left(\frac{\tanh ^{-1}\left(\frac{\sqrt{K \cdot k^{\prime}} n}{\sqrt{k \cdot p}}\right)}{\sqrt{k \cdot k^{\prime} \cdot K \cdot p_{H 2}}}-\frac{\log \left(k \cdot p_{H 2}-k^{\prime} \cdot K \cdot n^{2}\right)}{2 \cdot k^{\prime}}\right)+I_{C}
$$

where $I_{C}$ is the constant of integration.

With equation (2.9) the absorption time can be calculated depending on temperature, hydrogen partial pressure and sample thickness. The absorption time at $90^{\circ} \mathrm{C}$, and 50 mbar hydrogen partial pressure for a $100 \mu \mathrm{m}$ palladium foil is around 20 minutes and given in Figure 2.2. The results of equation (2.9) can be well approximated with an exponential decay function in the pressure and temperature range relevant for this thesis $\left(R^{2}>0.999\right)$.

\subsubsection{The phase diagram}

The palladium-hydride occurs in two different phases below the critical temperature of $293^{\circ} \mathrm{C}$ [98]: the $\alpha$ - and $\beta$-phase. The formation of the phases depend 


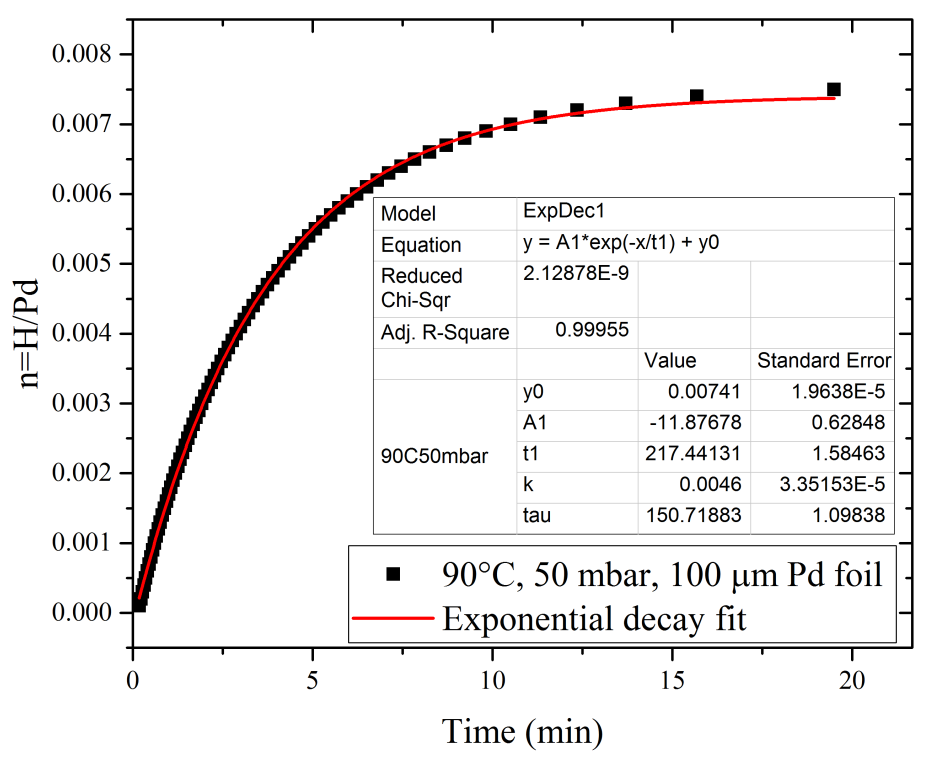

Figure 2.2: Absorption time calculated with equation (2.9) at $90^{\circ} \mathrm{C}$, and $50 \mathrm{mbar}$ hydrogen partial pressure for a $100 \mu \mathrm{m}$ palladium foil.

on the hydrogen partial pressure, the temperature and the mole ratio of hydrogen to palladium $n=\mathrm{H} / \mathrm{Pd}$. The phase diagram with several isotherms is given in Figure 2.3.

The $\alpha$-phase forms at low $n$ and exists exclusively up to $n=\alpha_{\max } \cdot \alpha_{\max }$ increases with increasing temperature. Above $\alpha_{\max }$, the $\beta$-phase starts to form and coexist. In this so called miscibility gap, $n$ increases at constant hydrogen partial pressure and temperature up to $n=\beta_{\min }$. At $\beta_{\min }$ the $\beta$-phase is exclusively present and $n$ increases with increasing hydrogen partial pressure at constant temperature. [7]

Both, the $\alpha$ - and $\beta$-phase, are solid phases. However, they can be distinguished by the lattice constant. The lattice constant for palladium is $3.890 \AA$ $(n=0)$, in the $\alpha$-phase it is $3.894 \AA\left(n=\alpha_{\max }\right)$, and in the $\beta$-phase it is 4.025 $\AA\left(n=\beta_{\text {min }}\right)$. The increased lattice constant causes an increased hydrogen solubility, which explains the increase of $n$ at constant hydrogen partial pressure and temperature in the miscibility gap. [7]

Above the critical temperature, there is no distinction between the two phases and the hydride exists in a homogeneous solid solution $[7,98]$. Below the critical temperature hysteresis can be observed, when $n=\alpha_{\max }$ is exceeded. The hysteresis is an effect from the extensive deformation due to the large volume changes during the phase change [98]. This deformation can be severe and a significant, irreversible plastic deformation of palladium foil specimen 


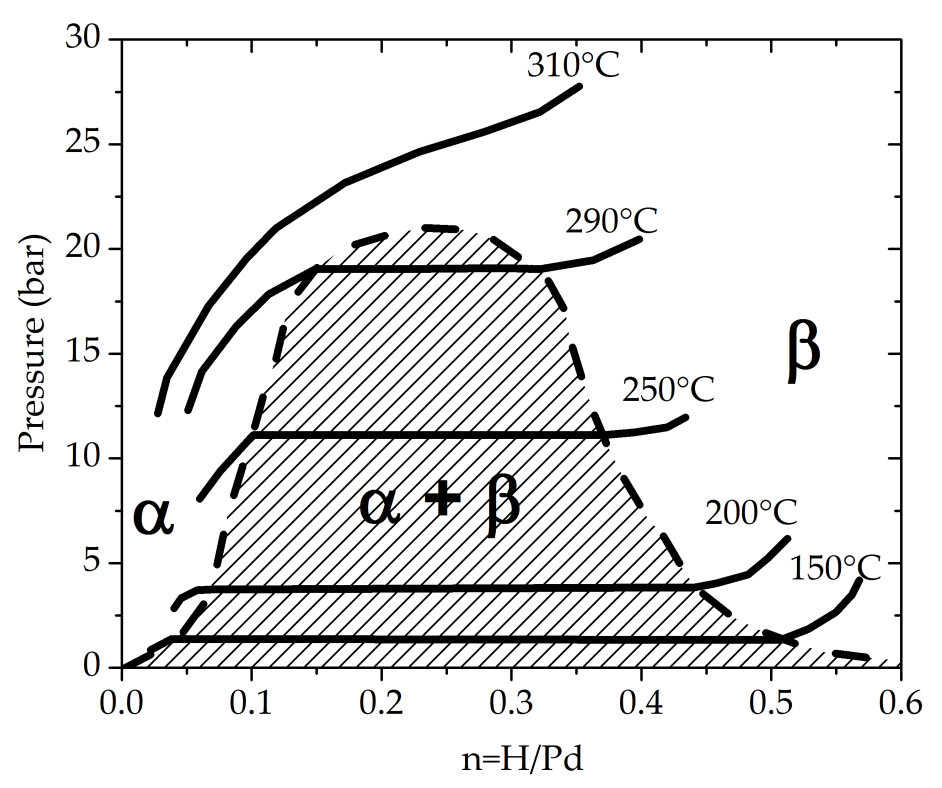

Figure 2.3: Phase diagram. Adapted from [82].

after several cycles between the phases has been observed [82]. If the phase change is avoided, the absorption process is fully reversible [82].

The phase change and the irreversible deformation can be avoided by alloying certain metals [82], e.g., for palladium silver with an silver content larger than $30 \%$ the phase change is avoided at normal temperatures. The critical temperature is $144^{\circ} \mathrm{C}, 63^{\circ} \mathrm{C}$, and $-91^{\circ} \mathrm{C}$ for 10,20 and $30 \%$ alloyed silver respectively. However, hysteresis can still be observed at higher silver contents [25].

\subsubsection{Hydrogen solubility in the $\alpha$-phase}

A large body of work has been done on experimentally determining the correlation between pressure, temperature and the ratio $n$ predominantly for high pressures and temperatures $[6,7,82,98]$. Two main studies can be cited describing the $\alpha$-phase at temperatures below $100^{\circ} \mathrm{C}[126,144]$. Selected results are shown in Figure 2.4.

One approach to calculate $n$ is via Sieverts's law, which describes the gas solubility in metals [24] and is

$$
\sqrt{P_{\mathrm{H}_{2}}}=K \cdot n
$$

where $p_{\mathrm{H}_{2}}$ is the pressure in torr and $K$ is the Sieverts's constant. $K$ at $30^{\circ}, 60^{\circ}$, and $90^{\circ}$ derived from experimental data is 353,500 , and 669 torr $^{0.5}$ respectivly [25]. However, Sieverts's law is only valid at very low ratios $n[25,126,144]$. 


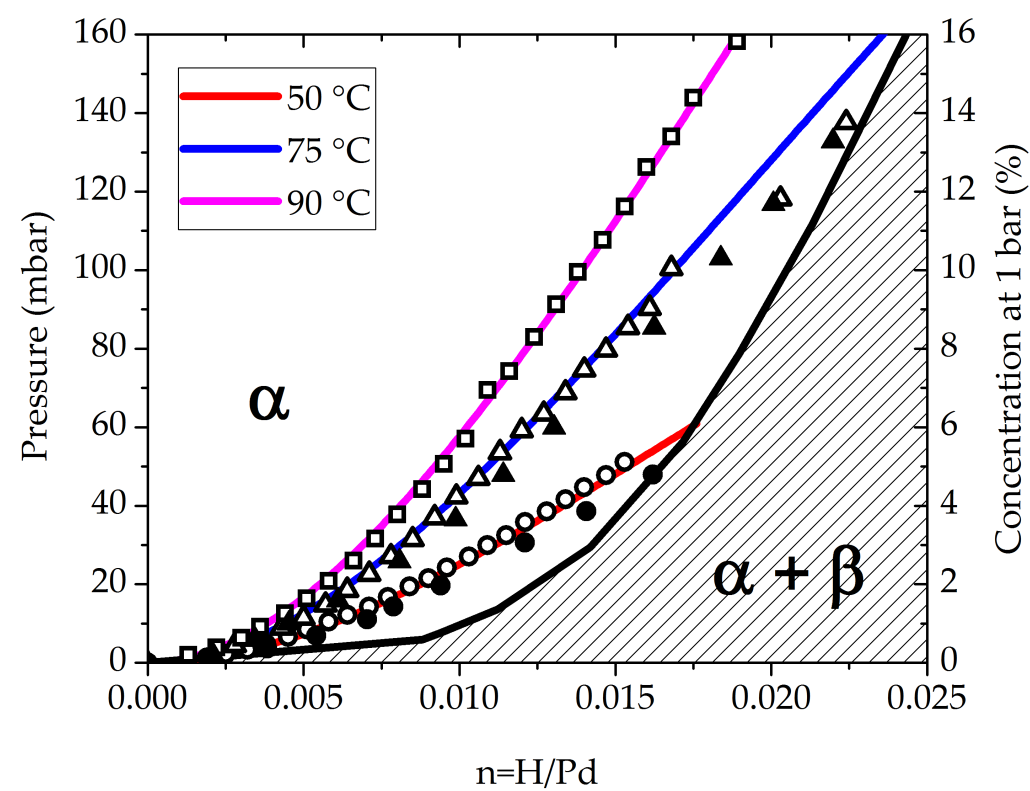

Figure 2.4: Phase diagram at low temperatures and low pressures. With experimental data from Wicke and Nernst at $50^{\circ} \mathrm{C}(\bullet)$, and $75^{\circ} \mathrm{C}(\boldsymbol{\Delta})$, and from Simons and Flanagan at $50{ }^{\circ} \mathrm{C}(\circ), 75^{\circ} \mathrm{C}(\triangle)$, and $90^{\circ} \mathrm{C}(\square)[126,144]$. Also displayed as lines are calculated results using (2.11).

Larcher developed a more complex thermodynamic model for the hydrogen solubility [78]. Simons and Flanagan adapted the model to fit their experimental results. The equation obtained is

$$
\ln (p)=13.04-\frac{2327}{T}-\frac{11110 \cdot n}{T}+2 \cdot \ln \left(\frac{n}{1-n}\right)
$$

where $p$ is the pressure in atmosphere, and $T$ is the temperature in Kelvin. The results of the calculation are shown in Figure 2.4 and describe the experimental results well. Furthermore, to compare (2.10) and (2.11) the results of both equations for different temperatures are shown in Figure 2.5. The results of both equations clearly diverge for $n>0.010$. Nevertheless, Sieverts's law seems to be an appropriate approximation solution up to $n=0.010$ as the deviation is small, however it is inaccurate for $n>0.010$.

Certain palladium alloys have a higher hydrogen solubility (higher $n$ at constant temperature and hydrogen partial pressure) than pure palladium [82]. Palladium silver for example, has an increased hydrogen solubility at low $n$ $\left(n<0.25\right.$, silver $<30 \%, 50^{\circ} \mathrm{C}$ ) [25]. The increase in solubility can be quantified with (2.10) and Sieverts's constant for different alloys. In Table 2.2 Sieverts's constants for palladium silver alloys with different silver content are listed. Sieverts's constant decreases with increasing silver content which means $n$ and 


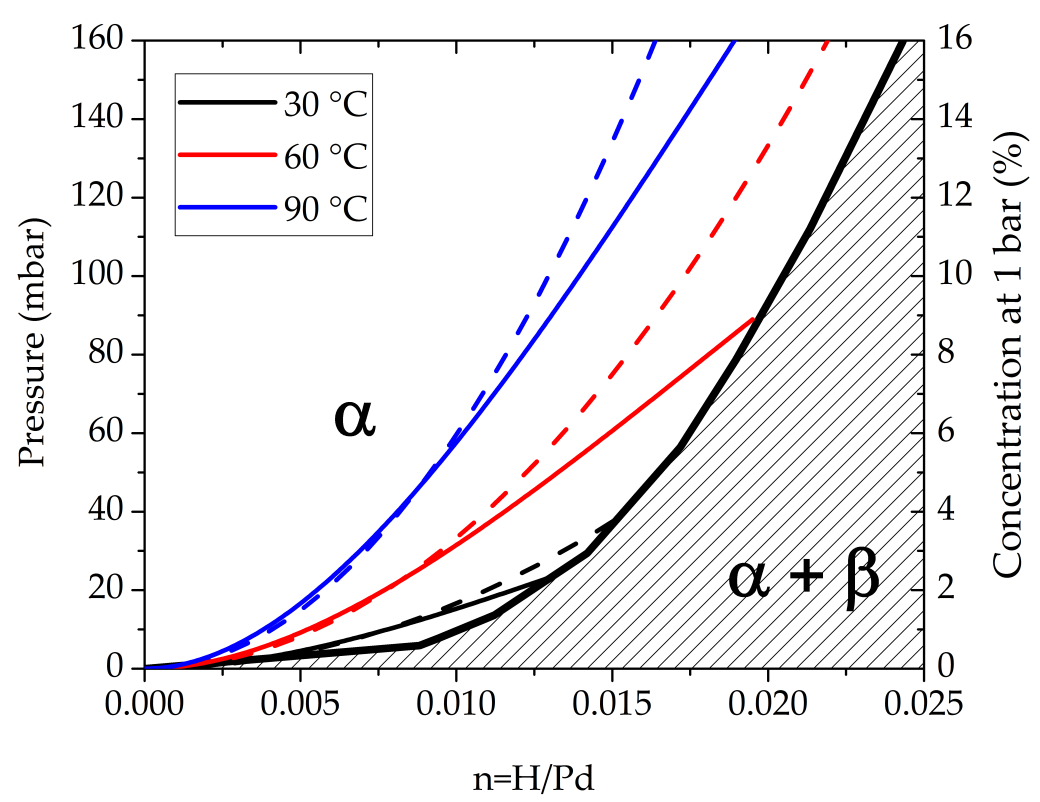

Figure 2.5: Phase diagram. Calculated values according to (2.10) (dashed lines) and (2.11) (solid lines) at $30^{\circ} \mathrm{C}, 60^{\circ} \mathrm{C}$, and $90^{\circ} \mathrm{C}$.

Table 2.2: Sieverts's constant of palladium with different silver content at $30^{\circ} \mathrm{C}$, $60^{\circ} \mathrm{C}, 90^{\circ} \mathrm{C}$ derived from [25].

\begin{tabular}{lrrr}
\hline Temperature & $30^{\circ} \mathrm{C}$ & $60^{\circ} \mathrm{C}$ & $90^{\circ} \mathrm{C}$ \\
\hline Pd & 353.2 & 500.3 & 668.6 \\
Pd90/Ag10 & 49.0 & 93.5 & 160.5 \\
Pd80/Ag20 & 20.5 & 41.3 & 74.2 \\
Pd70/Ag30 & 8.5 & 19.4 & 39.0 \\
\hline
\end{tabular}

the solubility increase with increasing silver content.

\subsubsection{Changes in material properties}

With the absorption of hydrogen, the palladium lattice expands and the material properties are changed.

The volumetric expansion is linear over a large ratio $n(n<0.7)$ and relatively independent of alloying materials (silver, platinum, copper, irridium, gold) [16]. Alefeld compares several studies and finds that the relative volumetric expansion is well described by

$$
\frac{\Delta V}{V}=n(0.19 \pm 0.01)
$$


Furthermore, with hydrogen absorption the optical, mechanical, and electrical properties of palladium are changed, e.g., the reflectance decreases, whereas the transmission and refractive index increase with increasing hydrogen absorption [14]. The Young's modulus decreases and the thermal expansion coefficient increases with increasing hydrogen absorption [61,82]. And finally, the electric resistivity increases with increasing hydrogen absorption [82].

\subsubsection{Inhibiting gases}

Palladium selectively absorbs hydrogen [3,4]. However, several other gases can have an influence on the adsorption of hydrogen on the palladium surface and therefore inhibit the absorption. The following gases have been reported to have an effect on the hydrogen absorption: $\mathrm{O}_{2}, \mathrm{CO}, \mathrm{CO}_{2}, \mathrm{H}_{2} \mathrm{O}, \mathrm{H}_{2} \mathrm{~S}$, and $\mathrm{SO}_{2}[5,9,10,33,39,58,69,105]$. Of those $\mathrm{CO}$ and $\mathrm{H}_{2} \mathrm{~S}$ have the most significant impact, but normally only $\mathrm{CO}$ is present in a transformer.

Surface investigations at ultra high vacuum (UHV) and low temperatures have shown that $\mathrm{CO}$ adsorbs to the palladium surface occupying palladium atoms and blocking hydrogen dissociation sites [76]. In addition, the sticking coefficient (ratio of adsorbed to impinged molecules [52]) of $\mathrm{CO}$ is close to 1 whereas that of hydrogen is 0.5 to 0.7 at temperatures below $125 \mathrm{~K}$ [35]. Therefore, the surface coverage of $\mathrm{CO}$ is increased compared to hydrogen. The adsorption of $\mathrm{CO}$ has been shown to increase with increasing pressure and decreasing temperature $[38,128,148]$. This correlates with a decreasing sticking coefficient with increasing temperature [17].

In general, the extrapolation from UHV and low temperature experiments to high pressure and room temperature is difficult [128]. However, for sensing applications the impact of $\mathrm{CO}$ can still be observed at temperatures above $25^{\circ} \mathrm{C}[51,69]$. Jayaraman showed that the sensor response is slowed down but the overall response/ signal is not attenuated and this effect could be further reduced with increasing the temperature [69]. On the other hand, Eriksson reported that $\mathrm{CO}$ completely blocks the absorption of hydrogen in palladium leading to an unresponsive sensor [51].

\subsection{Review of hydrogen sensors}

In laboratories hydrogen is usually detected using gas chromatographs and mass spectrometers. These are large, expensive instruments, and need regular maintenance [65]. Therefore, they are not suitable for implementation in distribution transformers and are not elaborated further. Smaller hydrogen sensors 
can be grouped into eight categories according to their working principle [65]: Catalytic, thermal conductivity, electrochemical, resistance based, work function based, mechanical, optical and acoustic. In this chapter we explain the working principles of the different categories and then concentrate on the approaches which are used in combination with fiber optic sensors.

\subsubsection{Working principles}

The eight different working principles are as follows [65]:

1. Catalytic sensors work on the principle that an exothermic reaction on the catalytic material of the sensor releases heat. This heat causes a change in temperature which is detected. The chemical reaction is the oxidation of hydrogen to water.

2. Thermal conductivity sensors work on the principle of a hot body's heat loss to its surroundings. The heat loss depends on the thermal conductivity of the surrounding gas which again depends on its composition. A selective membrane or column and a reference sensor are necessary for accurate detection of hydrogen.

3. Electrochemical sensors work on the principle of using a catalytic electrode to break molecular hydrogen into atomic hydrogen and free electrons. The atomic hydrogen forms water with oxygen and the free electrons cause a current flow.

4. Resistance based sensors can be divided into semiconducting metal-oxide sensors and metallic resistor sensors:

- Semiconducting metal-oxide sensors work on the principle that their electrical properties change when exposed to hydrogen. Molecular oxygen and hydrogen are adsorbed at the metal-oxide surface and form water. Free electrons are released and donated to the metaloxides surface leading to a decrease in resistivity.

- Metallic resistor sensors work on the principle that the electrical resistivity of palladium increases due to hydrogen absorption.

5. Work function based sensors work on the principle that diffused hydrogen adsorbs on a metal-oxide layer and changes the work function. This change can be detected by a voltage change. 
6. Mechanical sensors work on the principle of the expansion of certain metals due to hydrogen absorption. The expansion is transduced into a strain sensor.

7. Optical sensors work on the principle of the optical property change of certain materials when interacting with hydrogen

8. Acoustic sensors work on the principle of changes in the acoustic properties of a material due to the adsorption of hydrogen on the surface or absorption of hydrogen in the material.

All of the presented sensors detect hydrogen indirectly. Palladium, platinum and a variety of oxidized metals are a suitable sensing material. The sensing material interacts with hydrogen and causes a change in temperature or mechanical, optical or electrical properties. Those changes are detected using a transducer [65].

Commercial DGA solutions use thermal conductivity, electro chemical, or resistance based sensors (see Table 2.1). Of these only the resistance based sensors can detect hydrogen directly in the oil. The other two can detect hydrogen only in the gas phase and therefore need an additional hydrogen selective membrane.

\subsubsection{Fiber optic based hydrogen sensors}

The first fiber optic hydrogen sensor was reported by Butler in 1984 [29]. Since then a vast number of sensors has been presented. Several review papers give a good overview of the field $[45,125,157]$. Fiber optic hydrogen sensors generally have the advantage of being insensitive to electro magnetic interference, intrinsically safe in explosive gas mixtures, and to some degree, offering the potential of distributed sensing [65]. Fiber optic hydrogen sensors can be allocated to three of the aforementioned working principles: Catalytic, mechanical and optical sensors.

Catalytic sensors use a temperature sensitive optical fiber sensor (OFS) coated with a reactive surface (e.g., tungesten oxide). The surface causes an exothermic reaction of hydrogen and oxygen. The generated heat, causes a temperature rise, which is measured with an OFS $[32,42,150]$.

Optical sensors measure the optical property changes of a material, commonly palladium with an OFS. The fiber is usually coated on a tapered section or on the fiber tip allowing the light to interact with a hydrogen sensitive material. The measurement principle is intensity based. $[18,19,30,34,37,87-89,97$, $107,110,118,119,129,135,137-139,143,149,153,154,156]$. 
Mechanical sensors measure the expansion of palladium (alloys) due to hydrogen absorption with a strain sensitive OFS. In literature mainly FBG based sensors are reported but also Fabry-Pérot type sensors have been evaluated $[8,26,27,29,36,37,40,41,43,44,70,71,75,79,80,90-95,109,114,116,124,127$, $131,133,141,146,151,158]$.

Table 2.3 lists the majority of reported hydrogen sensor types that rely on a FBG measuring the volumetric expansion of palladium alloys. For the mechanical sensors two basic principles can be distinguished: coating the fiber with a thin palladium layer, and adhesively bonding a large palladium body (wire, tube, foil) to the fiber. Most of the early reported sensors rely on the latter (see Table 2.3). The high sensitivity of these sensor concepts is accompanied by a relatively slow response. In recent years researchers have focused on sensor concepts with thin films. To still achieve a high hydrogen sensitivity with thin films, the fiber diameter is often altered by etching or side polishing. This increases the strain sensitivity of the FBG, but has the disadvantage of strain concentrations, which results in increased fragility [44].

The first application of a fiber optic based hydrogen sensor in transformer oil was performed by Butler in 1996 [28]. In recent years, several different fiber optic hydrogen sensors have been reported for this application, most of which rely on a FBG and palladium $[70,71,90-93,97,116]$. Here as well, reducing the fiber diameter is succeffully deployed to increas the hydrogen sensitivity [70, 90]. However, the structural integrity and long term stability of these fragile sensors is questionable. Using an adhesively bonded palladium body, instead of a thin palladium coating, has not been examined for dissolved hydrogen sensing in transformer oil.

\subsection{The fiber Bragg grating}

Fiber Bragg gratings (FBG) are OFS that are sensitive to strain and temperature. FBGs, have the advantage of being small, lightweight, intrinsically safe in combustible gases, insensitive to electromagnetic noise, able to tolerate harsh environments, and having the capability of quasi distributed sensing, meaning that several sensors can be implemented in a single optical fiber $[64,108]$. FBG sensors and FBG interrogating systems were commercialized decades ago which makes their application economically viable and the sensing concept proved to be robust.

A grating is written into the core of an optical fiber mainly via interferometric, phase-mask or point-by-point techniques. The grating is a section of periodic modulation of the refractive index (Figure 2.6). At the sections of 
Table 2.3: Summary of palladium based hydrogen sensors using FBG. Temperature in ${ }^{\circ} \mathrm{C}$, Sensitivity in $\mathrm{pm} / \%$, Response time. Values with $\left.{ }^{*}\right)$ are estimated using equations describe in Chapter 4.

\begin{tabular}{|c|c|c|c|c|c|c|}
\hline Concept & Material \& geometry & Temp. & Sens. & Res. & Year & Ref. \\
\hline Etched FBG $(30 \mu \mathrm{m})$ & $560 \mathrm{~nm}$ Pd coating & RT & 20 & - & '99 & [127] \\
\hline FBG & $15 \mu \mathrm{m} \mathrm{Pd}$ coating & 95 & 200 & $10 \mathrm{~m}$ & '99 & [109] \\
\hline FBG & $\begin{array}{l}\text { D- shaped Pd wire } \\
(\oslash 1 \mathrm{~mm})\end{array}$ & RT & - & - & '99 & [80] \\
\hline FBG & $\begin{array}{l}\text { Pd Tube } \\
\text { (33 } \mu \mathrm{m} \text { thickness) }\end{array}$ & 95 & 300 & $1 \mathrm{~h}$ & '99 & [131] \\
\hline Side polished FBG & $\begin{array}{l}\text { Pd foil } \\
\text { (2 } \mu \mathrm{m} \text { thickness) }\end{array}$ & 80 & 290 & $2 \mathrm{~m}$ & '04 & [79] \\
\hline FBG & $350 \mathrm{~nm}$ Pd coating & 20 & 25 & $10 \mathrm{~s}$ & '07 & [26] \\
\hline $\begin{array}{l}\text { FBG with mechanical } \\
\text { amplification }\end{array}$ & $\begin{array}{l}\text { Pd Tube } \\
(300 \mu \mathrm{m} \text { thickness) }\end{array}$ & 85 & $1300^{*}$ & $3 d$ & '07 & [94] \\
\hline Side polished FBG & $\begin{array}{l}110 \mathrm{~nm} \mathrm{WO}_{3} \mathrm{Pd} \text { com- } \\
\text { posit coating }\end{array}$ & RT & 5 & $10 \mathrm{~m}$ & '11 & [40] \\
\hline Side polished FBG & $\begin{array}{l}110 \mathrm{~nm} \mathrm{WO}_{3} \mathrm{Pd} \text { com- } \\
\text { posit coating }\end{array}$ & RT & 5 & $10 \mathrm{~m}$ & '11 & [40] \\
\hline Etched FBG $(17 \mu \mathrm{m})$ & $\begin{array}{l}110 \mathrm{~nm} \mathrm{Pd} / \mathrm{Ni} \text { com- } \\
\text { posit coating }\end{array}$ & 23 & 15 & $5 \mathrm{~m}$ & '12 & [43] \\
\hline FBG & $560 \mathrm{~nm}$ Pd coating & RT & 60 & $5 \mathrm{~m}$ & '12 & [92] \\
\hline Etched FBG $(38 \mu \mathrm{m})$ & $\begin{array}{l}100 \mathrm{~nm} \mathrm{Pd} / \mathrm{Ag} \text { com- } \\
\text { posit coating }\end{array}$ & 15 & 6 & $5 \mathrm{~m}$ & '13 & [44] \\
\hline Tapered FBG $(50 \mu \mathrm{m})$ & 150 nm Pd coating & RT & 80 & $2 \mathrm{~m}$ & '13 & [124] \\
\hline Etched FBG $(63 \mu \mathrm{m})$ & $500 \mathrm{~nm}$ Pd coating & 25 & 60 & $6 s$ & '13 & [114] \\
\hline Etched FBG $(21 \mu \mathrm{m})$ & $\begin{array}{l}130 \mathrm{~nm} \mathrm{Pd} / \mathrm{Ni} \text { com- } \\
\text { posit coating }\end{array}$ & 25 & 30 & $15 \mathrm{~m}$ & '14 & [41] \\
\hline FBG & 400 nm PdAg coating & 80 & $30^{*}$ & $20 \mathrm{~m}$ & '15 & [91] \\
\hline Microstructured FBG & 520 nm PdAg coating & 25 & 5 & $2 \mathrm{~m}$ & '15 & [75] \\
\hline Side polished FBG & 400 nm PdAg coating & RT & $275^{*}$ & $4 \mathrm{~h}$ & '15 & [71] \\
\hline Etched FBG $(50 \mu \mathrm{m})$ & 150 nm Pd coating & $\mathrm{RT}$ & 40 & $30 \mathrm{~m}$ & '15 & [36] \\
\hline Microstructured FBG & 520 nm PdAg coating & RT & 38 & $3 \mathrm{~m}$ & '16 & [158] \\
\hline FBG & 675 nm PdCr coating & $\mathrm{RT}$ & $12^{*}$ & $10 \mathrm{~m}$ & '16 & [116] \\
\hline Side polished FBG & 400 nm PdAg coating & RT & $279^{*}$ & $5 \mathrm{~h}$ & '16 & [90] \\
\hline Microstructured FBG & 500 nm PdAg coating & RT & 16 & $2 \mathrm{~m}$ & '16 & [160] \\
\hline Etched FBG $(16 \mu \mathrm{m})$ & 800 nm PdNi coating & RT & 125 & $5 \mathrm{~m}$ & '17 & [36] \\
\hline
\end{tabular}




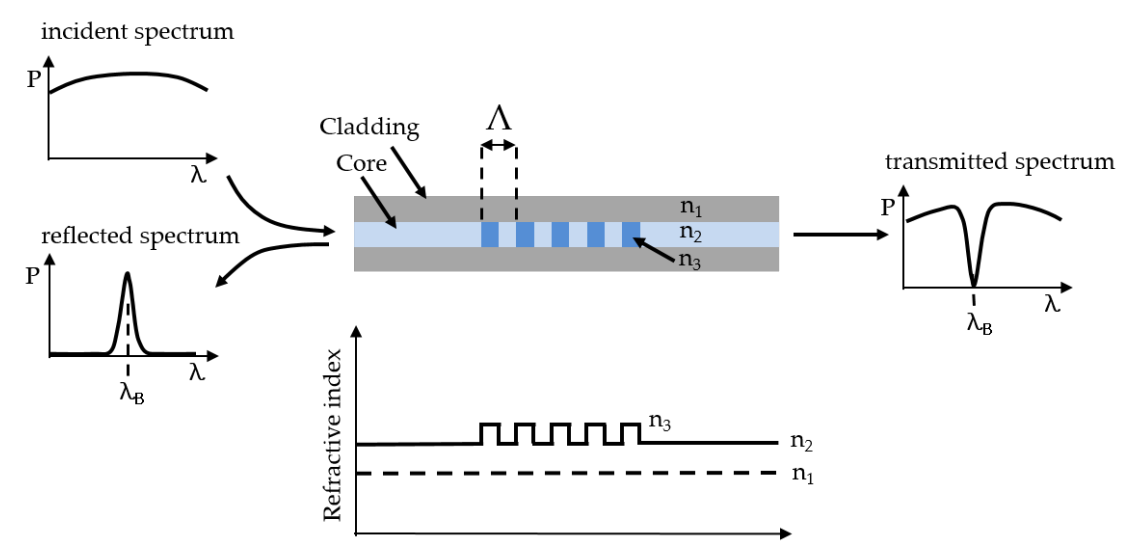

Figure 2.6: Fiber Bragg grating.

changed refractive index, light is successively refracted and reflected. Light fulfilling the Bragg condition (2.13) has the strongest reflection as each reflection is in phase.

$$
\lambda_{B}=2 n_{\mathrm{eff}} \Lambda
$$

where $\lambda_{B}$ is the peak wavelength of the back reflected spectrum, $n_{\mathrm{eff}}$ is the effective refractive index and $\Lambda$ is the period of the grating.

The peak wavelength of the reflected light is influenced by changes of the fiber properties, e.g. temperature and strain. Temperature changes impact the effective refractive index of the fiber, but also the grating period due to thermal expansion. Strain mainly affects the grating period, but also changes the effective refractive index. The impact on the peak wavelength due to temperature and strain changes is

$$
\Delta \lambda_{B}=2\left(\Lambda \frac{\delta n_{\mathrm{eff}}}{\delta \varepsilon}+n_{\mathrm{eff}} \frac{\delta \Lambda}{\delta \varepsilon}\right) \Delta \varepsilon+2\left(\Lambda \frac{\delta n_{\mathrm{eff}}}{\delta T}+n_{\mathrm{eff}} \frac{\delta \Lambda}{\delta T}\right) \Delta T
$$

(2.14) can be simplified to

$$
\Delta \lambda_{B}=k_{\varepsilon} \Delta \varepsilon+k_{T} \Delta T
$$

where $k_{\varepsilon}$ is the strain sensitivity, $k_{T}$ is the temperature sensitivity, and $\Delta \varepsilon$ and $\Delta T$ are the change in strain or temperature of the fiber. The values for $k_{\varepsilon}$ and $k_{T}$ can be approximated by (2.16) and (2.17) [64]:

$$
\begin{aligned}
& k_{\varepsilon} \approx \lambda_{B} 0.78 \cdot 10^{-6} \frac{1}{\mu \varepsilon} \\
& k_{T} \approx \lambda_{B} 6.7 \cdot 10^{-6} \frac{1}{{ }^{\circ} C}
\end{aligned}
$$

where $\mu \varepsilon$ is microstrain $\left(\mu \varepsilon=10^{-6} \Delta l / l\right)$.

For a peak wavelength of $1550 \mathrm{~nm}$ the equations lead to $k_{\varepsilon} \approx 1.21 \mathrm{pm} / \mu \varepsilon$ and $k_{\mathrm{T}} \approx 10.4 \mathrm{pm} /{ }^{\circ} \mathrm{C}$. 


\subsubsection{Application of FBG sensors}

In addition to the application as hydrogen sensors, FBGs have been used as strain and temeprature sensors for different applications for decades [108].

The primary application of FBG lies in structural monitoring of large civil structures (e.g., bridges, tunnels) $[96,108]$. Another application is in monitoring the load in wind turbine blades [117]. However, FBGs are also popular in the aerospace industry since they are insensitive to electro magnetic interference, light weight (multiplexable), and intrinsically safe in combustible gases. They are also used in composite structures to monitor stress and strain, which allows active damping, profiling of heat shields, and the impact detection of debrids $[21,74,108]$. Furthermore, FBGs are used in marine applications. Here they are used for strain monitoring of fiber composite lock gates and composite hulls, and temperature monitoring in bore-holes [108]. There is also ongoing research in FBG applications in railways and transmission lines, as well as in the medical and nuclear sectors [99, 108, 130,132]. Furthermore, FBGs show promising results as moisture sensors and in their application at low temperatures for the quench detection in superconductors $[86,152]$. 


\section{Chapter 3}

\section{Materials and Methods}

This chapter introduces the materials and methods used in this thesis. First, the relevant materials and methods for the sensor manufacture are described. Second, the optical measurement equipment is presented. Then, the signal processing approach is described. In this chapter also the test setups for hydrogen measurements in gas and oil are presented, as well as the equipment used for tensile tests.

\subsection{Material and Methods for Sensor Manufactur- ing}

This section describes the materials and methods used for the sensor manufacturing: (a) the commercial FBGs are discussed. (b) the etching process of the fiber, to achieve a reduced fiber diameter, is described. (c) the sputter deposition system to coat the fibers with a palladium film and palladium foils with silica films is described. (d) the adhesives used to attach palladium foils to the fibers are presented. (e) the properties of the commercial palladium foils are described.

\subsubsection{Fiber Bragg gratings}

Three different types of commercial FBG (silica-based, single-mode) sensors were used. The center wavelength was $1550 \pm 10 \mathrm{~nm}$. The grating length was $10 \mathrm{~mm}$. All fibers had a $125 \mu \mathrm{m}$ cladding diameter, but different coating materials: acrylate, polyimide, and organically modified ceramic (Ormocer). The acrylate and polyimide coated fiber were supplied by Beijing Samyon Instruments, Beijing. They have an apodized grating written in a section of a stripped fiber. The acrylate and polyimide fibers both were re-coated with acrylate (the 
re-coating increases the stability during shipping and handling), however, the re-coating was removed for sensor manufacture. The Ormocer fibers were supplied by FBGS, Jena. The draw tower gratings have improved mechanical stability and strain transfer from the coating into the fiber due to the Ormocer coating $[72,145]$. Draw tower gratings are written into the fiber core during the manufacturing (drawing) of the fiber itself. Therefore, no re-coating of the fiber is necessary.

\subsubsection{Fiber stripping}

To remove the the coating from the fiber, the fiber has to be stripped. Generally, three different methods are used [103]: mechanical, vaporization, and chemical stripping.

For the mechanical stripping a stripping tool, similar to a wire stripping tool, is used. This stripping process is fast, but the fiber can be easily damaged during the process $[103,147]$. For thermo-mechanical stripping a thermal stripping tool is used. The tool is similar to the non-thermal stripping tool, but additionally contains a heater that heats the coating until it can be easily removed. The mechanical stripping always degrades the fiber surface, which impacts the mechanical stability negatively.

For vaporization stripping a combustion process is used to remove the coating from the fiber. The vaporization is fast and safe, but the burning of the coating decreases the mechanical stability even more than the mechanical stripping [147].

For chemical stripping a solvent (dichloromethane) or an acid (concentrated sulfuric acid) is used [147]. This process is slower and needs longer setup time, but the fiber itself is unlikely to be damaged during the process. A further concern with chemical stripping is the health hazard of the acids and solvents used. Precautions have to be taken to minimize the risk when handling these chemicals.

In this thesis mechanical or chemical stripping were used to remove the coating material.

\subsubsection{Fiber etching}

To decrease the diameter of the fiber and therefore increase its strain sensitivity, the fiber can be chemically etched. Commonly, silica based optical fiber is etched with hydrofluoric acid or potassium hydroxide [15,111,155]. Since hydrofluoric acid poses severe health risks it was not used. The etching process with potassium hydroxide was as follows: The stripped fiber was immersed 

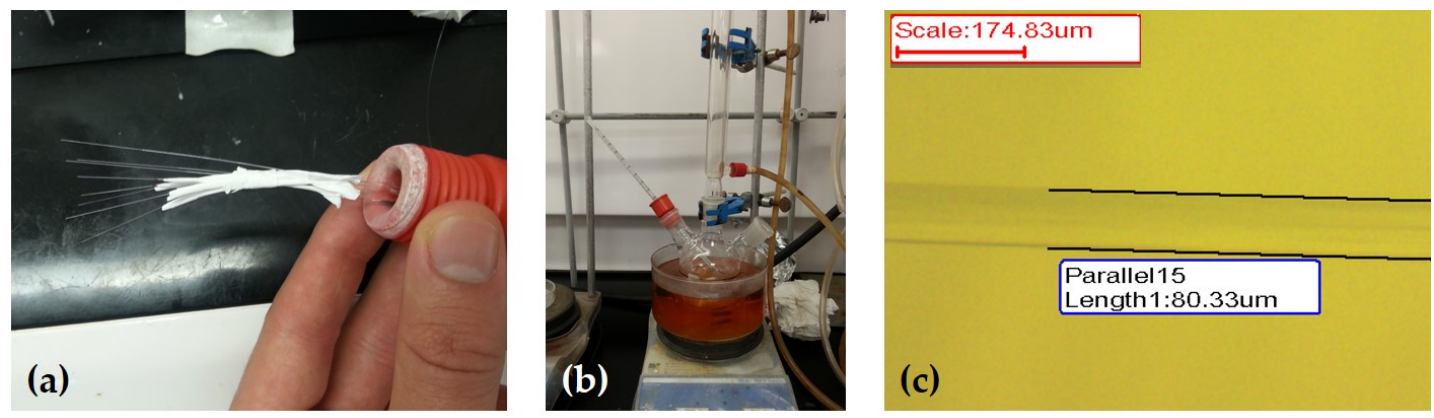

Figure 3.1: (a) Fiber before etching with PTFE protection.(b) Flask with potassium hydroxide sitting in heated oil bath.(c) Microscopic image of etched fiber.

Table 3.1: Parameters of the sputter deposition process for palladium, titanium, and silicon oxide.

\begin{tabular}{lrrrrr}
\hline Material & Power (W) & Voltage (V) & Current (A) & Pressure & Base pressure \\
\hline $\mathrm{Pd}$ & $175(\mathrm{DC})$ & $328-375$ & $0.467-0.534$ & $8 \cdot 10^{-3}$ Torr & $4.9 \cdot 10^{-8}$ Torr \\
$\mathrm{Ti}$ & $200(\mathrm{DC})$ & $355-377$ & $0.555-0.561$ & $3 \cdot 10^{-3}$ Torr & $4.9 \cdot 10^{-8}$ Torr \\
$\mathrm{SiO}_{2}$ & $100(\mathrm{RF})$ & $127-136$ & - & $3 \cdot 10^{-3}$ Torr & $1.2 \cdot 10^{-7}$ Torr \\
\hline
\end{tabular}

in potassium hydroxide ( $35 \% \mathrm{wt}$. $\mathrm{KOH}$ ) at $130^{\circ} \mathrm{C}$ (Figure $3.1(\mathrm{~b})$ ). The etching was monitored by repeated measurement of the diameter of a reference fiber under a microscope (Figure 3.1 (c)). The vapour also attacked parts of the fiber that were not immersed in the liquid. To protect these parts, the fiber were wrapped in PTFE sealant tape (Figure 3.1 (a)).

\subsubsection{Coating process - Sputter deposition}

Sputter deposition was used to coat palladium on the stripped fiber. The thin films were grown in an argon environment in a Kurt J Lesker CMS-18 UHV system. Three different materials were grown: Palladium, titanium, and silicon oxide. Titanium was used as an adhesion layer between fiber and palladium and silicon oxide was used to coat palladium foils with a membrane (see Chapter 4). The films were grown with the parameters given in Table 3.1.

Two holders were designed and manufactured to hold fibers and palladium foils in place during sputtering, and to enable coating from both sides (see Figure 3.2). Both holders have the outer dimension of a 4 inch silicon waver, with a cut out in the center. After coating one side, the holder can be easily turned around to coat the other side.

The fiber holder is made from two stainless steel sheets, which are con- 

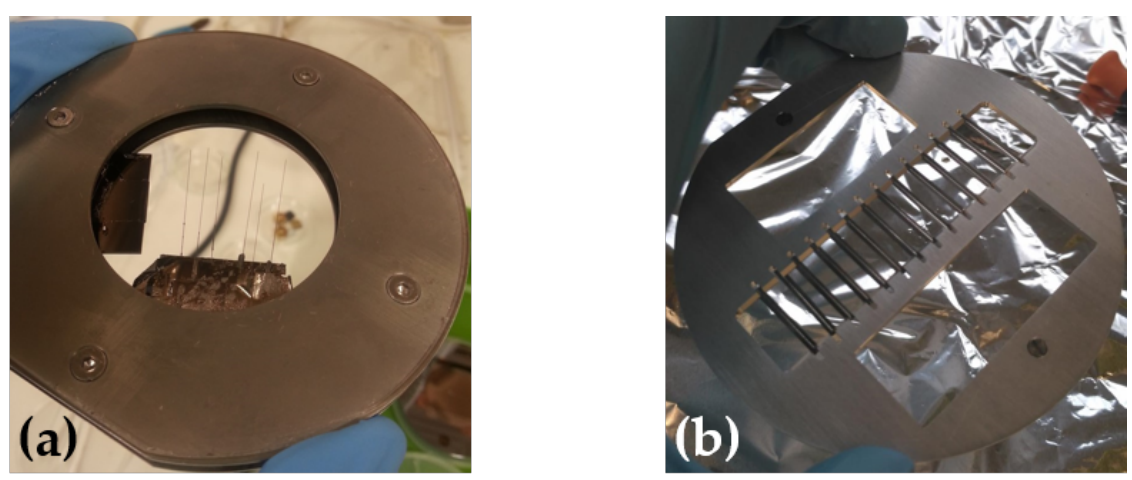

Figure 3.2: (a) Sputter holder with mounted fibers.(b) Sputter holder with mounted foils.

nected but separated at a defined distance of $5 \mathrm{~mm}$ via 4 screws and spacers (see Figure 3.2(a)). The etched fibers were first mounted on a glass slide with kapton tape. Then the glass slide was mounted on the first part of the holder with kapton tape. The excess fiber was also attached before the second part of the holder was connected.

The foil holder is made from aluminium and can hold up to fourteen 100 $\mu \mathrm{m}$ palladium foils ( $2 \mathrm{~mm} \times 20 \mathrm{~mm}$ ) in defined grooves (see Figure 3.2(b)). The two parts are connected via two screws and clamp the foils in between.

\subsubsection{Adhesives}

For bonding purposes four adhesives were used: $\mathrm{NOA} 83 \mathrm{H}$, and NOA86H (Norland Products Inc.), ADR 243 (Adhesive Technologies NZ Ltd.), and Loctite 401 .

The NOA83H is a single compound, mercapto-ester based adhesive and can be cured with ultraviolet light and heat. The adhesive starts curing at $60^{\circ} \mathrm{C}$ and can be fully cured in 10 minutes at $125^{\circ} \mathrm{C}$. After curing, the adhesive withstands temperatures up to $150^{\circ} \mathrm{C}$. However it has a glass transition temperature of below $45^{\circ} \mathrm{C}$.

The NOA86H is a single compound, acrylic based adhesive and has the same curing requirements as the NOA83H. After curing, the adhesive withstands temperatures up to $125^{\circ} \mathrm{C}$. The glass transition temperature is also below $45^{\circ} \mathrm{C}$.

The ADR 243 is a bisphenol A resin and was used with ADH 182, a high temperature hardener. The epoxy starts curing at room temperature and has a pot life of 6 hours after preparation. After curing for 3 hour at $100^{\circ} \mathrm{C}$ the heat deflection temperature is $135^{\circ} \mathrm{C}$.

The Loctite 401 is a single compound, cyanoacrylate based adhesive. The 
adhesive cures in seconds at room temperature and obtains full strength after a 24 hour cure at room temperature. The glass transition temperature is also below $121^{\circ} \mathrm{C}$.

Initially, the $\mathrm{NOA} 83 \mathrm{H}$ and $\mathrm{NOA} 86 \mathrm{H}$ were tested for the manufacturing of the hydrogen sensors (see Chapter 4). Both adhesives showed uncontrolled post-curing behaviour which resulted in inconsistent results (see Chapter 5). Furthermore, the adhesives have a glass transition temperature below $45^{\circ} \mathrm{C}$, which makes them unsuitable for applications above this temperature. Therefore, they were replaced with the ADR 243, which has a heat deflection temperature above the maximum working temperature $\left(120^{\circ} \mathrm{C}\right)$ of the sensor. Furthermore, no post-curing was observed with the ADR 243. For a different experiment in which FBGs are bonded to a tensile test bar the Loctite 401 is used since it cures quickly at room temperature and it was not exposed to high temperatures afterwards.

\subsubsection{Palladium foils}

The palladium foils used to manufacture the sensors were supplied by Goodfellow. The palladium has a $99.95 \%$ purity and foils in two thickness were used: 20 and $100 \mu \mathrm{m}$. The $20 \mu \mathrm{m}$ foil was supplied in a sheet $(100 \mathrm{~mm} \times 100 \mathrm{~mm})$ and the $100 \mu \mathrm{m}$ foil on a coil $(2 \mathrm{~mm} \times 1 \mathrm{~m})$. The palladium silver foil has a $75 \%$ palladium to $25 \%$ silver ratio, a thickness of $10 \mu \mathrm{m}$ and is supplied in a sheet ( $50 \mathrm{~mm} \times 50 \mathrm{~mm}$ ). The thickness of the foils was chosen depending on the desired sensor response (see Chapter 4 ) and the availability of commercial foils.

\subsection{Optical measurement equipment}

In this section the equipment used to interrogate the fibers is presented. The equipment includes an optical spectrum analyser (MS9740A, Anritsu), FBG interrogator (si155, Micronoptics), a SLD source (1550 nm, $22 \mathrm{~mW}, 50 \mathrm{~nm}$ bandwidth; S5FC1005S, Thorlabs), a DFB laser (1550 nm, $1.5 \mathrm{~mW}$; S3FC1550, Thorlabs), and fiber optic switches (GP700, DiCon).

The selection of the equipment for the individual experiments depended on availability. However, with the data processing approach described in Section 3.3 the measurements were largely independent of the equipment used. 


\subsubsection{Optical Spectrum Analyser - Anritsu MS9740A}

The optical spectrum analyser (OSA) model is a MS9740A by Anritsu. The OSA has a wavelength measurement range of 600 to $1700 \mathrm{~nm}$, and a wavelength accuracy of $\pm 20 \mathrm{pm}$. The OSA has an inbuilt light source which was only used for calibration purposes and not for interrogation of the FBG. Three different units have been used in the course of this thesis. The initial unit was found to be faulty and had a high wavelength inaccuracy. The wavelength inaccuracy could be correlated to room temperature fluctuations. During its repair two other loan units were used. However, using the DFB laser as a wavelength reference, reliable measurements could be achieved with all units (see Section 3.3.2).

\subsubsection{FBG Interrogator - Micronoptics si155}

The interrogator model is a si155 by Micronoptics. The interrogator has a wavelength measurement range of 1520 to $1580 \mathrm{~nm}$, and a wavelength accuracy of $\pm 1 \mathrm{pm}$. The interrogator has an integrated light source.

\subsubsection{Light Sources - Thorlabs S5FC1005S}

The light source is a superluminescent diode (SLD) model is a S5FC1005S by Thorlabs. The light source is power and temperature controlled, has a center wavelength at $1550 \mathrm{~nm}$, and a bandwidth of $50 \mathrm{~nm}$. The current was set to $500 \mathrm{~mA}$ and the temperature to $25.0^{\circ} \mathrm{C}$.

\subsubsection{Distributed feedback laser - Thorlabs S3FC1550}

The DFB laser model is a S3FC1550 by Thorlabs. The DFB laser is power and temperature controlled, and has a center wavelength at $1550 \mathrm{~nm}$.

\subsubsection{Fiber optic switch - DiCon GP700}

Two fiber optic switches model GP700 by DiCon were used. One switch has a module with 8 input channels, the other switch has two modules with 9 input channels.

\subsection{Data processing}

The inbuilt peak detection of the OSA and FBG interrogator can have difficulty detecting peaks that are separated by only hundreds of picometers and also 
peaks that are distorted (e.g.:unsymmetrical, peak split). The latter case occurs frequently with surface bonded FBGs. Furthermore, wavelength instability of the optical equipment due to temperature and pressure dependencies and the temperature cross-sensitivity of the FBG itself requires compensation. Therefore, a robust data processing approach was developed. Our data processing approach can be divided into peak detection and compensation.

\subsubsection{Peak detection}

For the OSA, a normalized spectrum is obtained through division of the reflected spectrum by the light source spectrum within the same wavelength range. This reduces the effect of the wavelength dependent intensity variations of the light source and is not necessary for the FBG interrogator. The resulting spectrum is then normalized to a range from 0 to 1 . Peak detection was performed using the LabVIEW peak detection algorithm with a quadratic fit $[22,104]$. The quadratic fit was performed over a spectral width of 200-300 pm, depending on the peak width.

\subsubsection{Compensation}

Wavelength inaccuracy of the optical measurement equipment can be compensated with the following three methods: (a) a wavelength stable laser (e.g., DFB laser), (b) a temperature controlled reference FBG, or (c) a reference FBG in combination with a non fiber optic temperature measurement (e.g., thermocouple, RTD). For (a) the peak wavelength of the laser is directly subtracted from the FBG peak wavelength. For (b) the peak wavelength of the temperature controlled reference FBG is directly subtracted from the peak wavelength of the hydrogen sensor. For (c) the measured temperature is multiplied by the temperature sensitivity of the reference FBG sensor (e.g., bare FBG) and the product is subtracted from the peak wavelength of the hydrogen sensor.

The temperature sensitivity of FBGs can be compensated for by: (a) a reference FBG $[62,159]$, or (b) a non-fiber optic temperature measurement. For (a) the peak wavelength of a reference FBG is divided by its temperature sensitivity (to calculate the temperature) and multiplied by the temperature sensitivity of the hydrogen sensor FBG. For (b) the measured temperature is multiplied by the temperature sensitivity of the hydrogen sensor FBG and the product is subtracted from the peak wavelength of the hydrogen sensor FBG.

In this thesis the following data processing approach is used (Figure 3.3a): The temperature in the chamber (measured with a thermocouple) was multiplied by the temperature sensitivity $k_{T}$ Sensor of each hydrogen sensor FBG and 


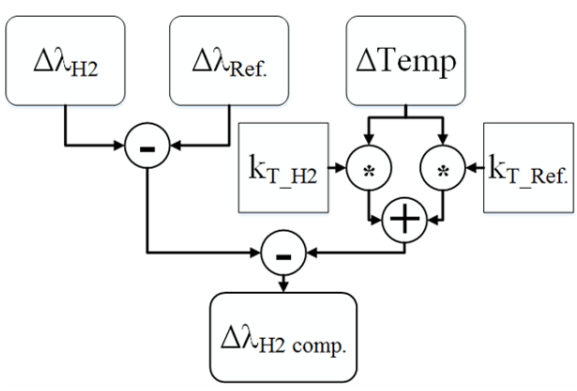

(a) For hydrogen measurement.

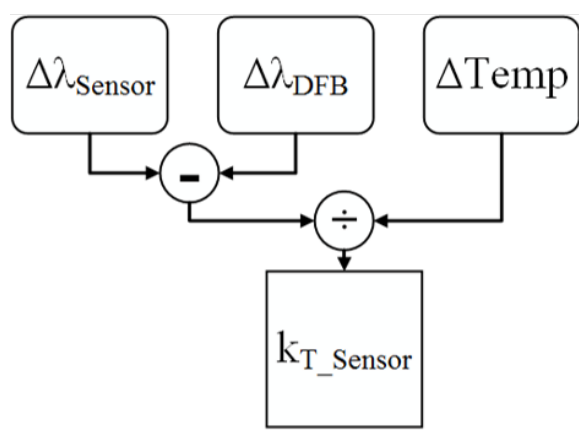

(b) For temperature measurement.

Figure 3.3: Data processing.

then subtracted from the corresponding peak wavelength. This compensates for fluctuations of the temperature in the test chamber. The peak wavelength of the reference FBG is also temperature compensated and then subtracted from the hydrogen sensor. This compensates for fluctuations of the optical components. The result is the compensated peak wavelength of the $\mathrm{H}_{2}$ sensors $\Delta \lambda_{\mathrm{H} 2 \text { comp. }}$

The temperature sensitivity of the FBGs was determined in the test setup during cool down from elevated to room temperature. If the OSA was subject to strong wavelength inaccuracy, the following approach was used(Figure 3.3b): The peak wavelength of a DFB laser was subtracted from that of the FBG sensors. This compensates for fluctuations of the optical components and the temperature sensitivity $k_{T}$ sensor of each sensor can be calculated.

With the data processing approach a wavelength accuracy of \pm 3 pm was achieved. This value was determined over a period of 12 hour during which a sensor at stable conditions was interrogated.

\subsection{Test setups}

Two test setups for sensor characterization were developed that are stable for gas mixture, temperature, and pressure.

In previous works OFS gas sensors have been measured in open systems in which the gas concentration is controlled by mass flow controllers (MFC) and the gas is not recycled [27,127]. An open system offers the advantage of a constant gas concentration. Disadvantages of open systems are a high gas consumption, difficulty in mixing the gases, and running under, or over pressure. Maier et al. [95] used a closed system, in which the gas concentration was controlled by volume fractions and a fan caused movement of the gas in the chamber. Advantages of these system are: minimal gas consumption, 
Table 3.2: List of pre-mixed gas cylinder used.

\begin{tabular}{clr}
\hline Cylinder & Gas & Concentration \\
\hline 1 & Hydrogen & $2510 \pm 30 \mathrm{ppm} \mathrm{H}_{2}$ in $\mathrm{N}_{2}$ \\
2 & Hydrogen & $5.00 \pm 0.03 \% \mathrm{H}_{2}$ in $\mathrm{N}_{2}$ \\
3 & Hydrogen & $5.0 \pm 0.5 \% \mathrm{H}_{2}$ in $\mathrm{N}_{2}$ \\
4 & Nitrogen & $99.999 \% \mathrm{~N}_{2}$ \\
5 & Nitrogen & $99.99 \% \mathrm{~N}_{2}$ \\
6 & Dry Air & $21.0 \pm 1.0 \% \mathrm{O}_{2}$ in $\mathrm{N}_{2}$ \\
7 & Carbon monoxide & $10.00 \pm 0.06 \%{\mathrm{CO} \text { in } \mathrm{N}_{2}}$ \\
\hline
\end{tabular}

good mixing, and the possibility of running at under, or over pressure. Closed systems have the disadvantages of decreasing hydrogen concentration due to hydrogen absorption into the palladium and diffusion out of the system, as well as heating of the gas.

The setups developed in this thesis are closed systems in which the gas mixture was circulated between a large mixing chamber (15 liter) and a smaller, temperature controlled test chamber (0.5 -3 litre) (see Figure 3.4). The mixing chamber serves the purpose of preparing gas mixtures by pressure fraction and also of compensating for hydrogen concentration decrease due to absorption into the palladium. The setup can be evacuated to a pressure below 10 mbar and can operate between that value and an over pressure of 1.37 bar. For the experiments reported in this thesis, the pressure was set to $1060 \mathrm{mbar}$ in the test chamber (the expected average pressure in a distribution transformer). The chambers were filled with a pre-determined gas concentration that is circulated using a diaphragm pump. The gas mixtures are prepared from different cylinders listed in Table 3.2.

The newly developed systems combine the advantages of both open and closed systems: a constant gas concentration due to the large volume of the mixing chamber, good mixing of the gases, good temperature stability, low gas consumption, low safety risk and the possibility to run at over and under pressure.

The first setup is for measurement in gas only. The second setup is for measurement in gas and oil. The second setup is placed in a fume hood for safety consideration. Therefore, experiments to investigate the cross-sensitivity to carbon monoxide can be conducted in this setup. 


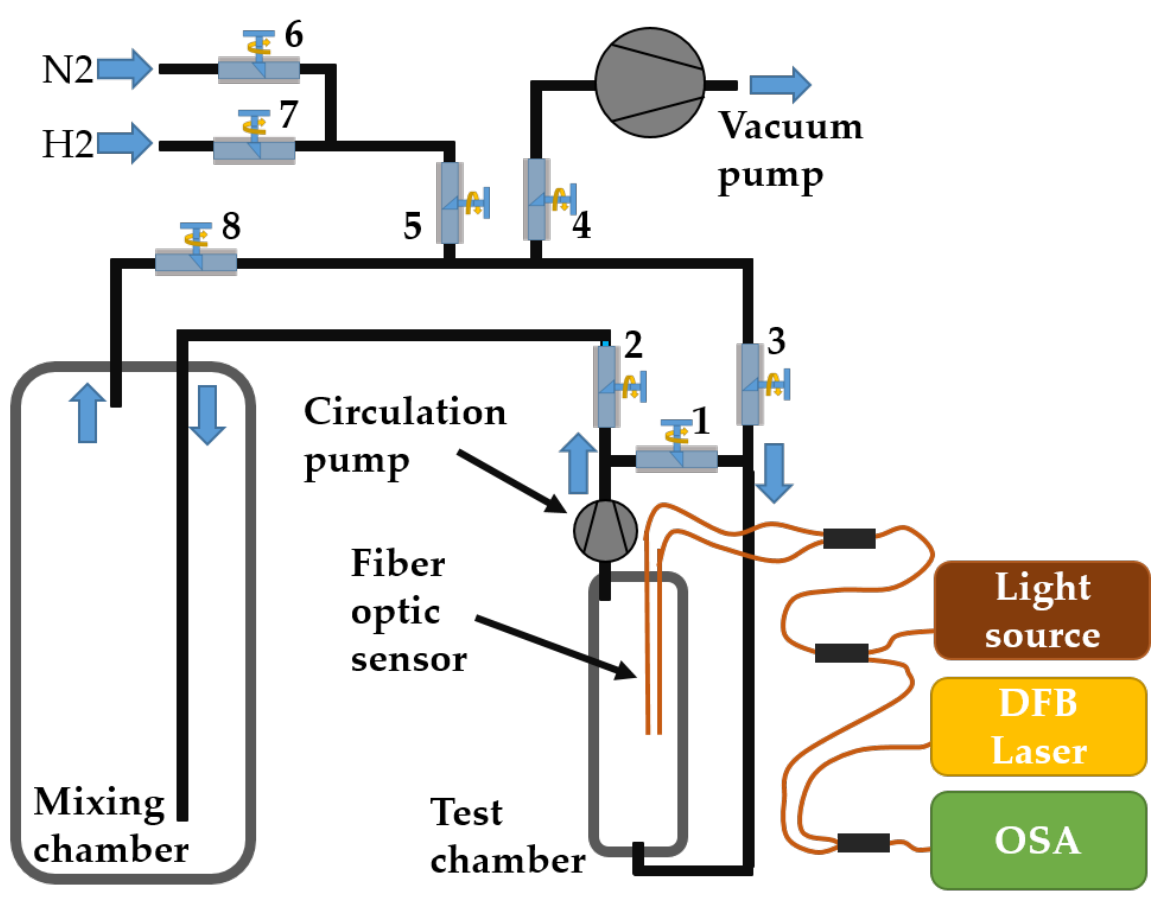

Figure 3.4: Schematic of setup I for testing sensors in gas.

\subsubsection{Setup I for measurements in gas}

The layout of setup I is shown in Figure 3.4. Two test chambers of different sizes were manufactured (see Figure 3.5). Initially, a test chamber with 0.5 litre volume was used. However it was found to be difficult to mount several sensors in the confined space (especially the pre-strained sensors) for simultaneous characterisation. For that reason, an additional, larger test chamber with 3 liter volume was designed.

The operation of the setup is as follows (subscripts $m$ and $t$ indicate the mixing chamber and test chamber respectively). To change the gases valve 1 was opened and then valve 2 and 3 were closed simultaneously. This enabled continuous testing in the test chamber while the gas was changed in the mixing chamber. Valve 4 was opened and the mixing chamber was evacuated $\left(p_{\mathrm{m}_{-} \mathrm{v}}<\right.$ 15 mbar). Valve 4 was closed and the mixing chamber was filled with a gas mixture up to $p_{\mathrm{m} \max }=1125 \mathrm{mbar}$ (this value is for the setup with the 0.5 litre test chamber) using valves 5, 6 and 7 . Afterwards, valve 5 and 8 are closed. Valve 3 and 4 were then opened to evacuate the test chamber $\left(p_{\mathrm{t}_{\mathrm{v}}}<15 \mathrm{mbar}\right.$ ). Finally, valve 4 was closed, valves 2 and 8 were opened and valve 1 was closed.

The final pressure in the mixing chamber is $1090 \pm 5$ mbar and in the test chamber $1060 \pm 5$ mbar (the pressure gradient is generated by the circulation pump). The gas mixtures are prepared by pressure fraction using the pressure gauges as an indicator. e.g., to achieve a $1 \%$ mix, the mixing chamber is first 


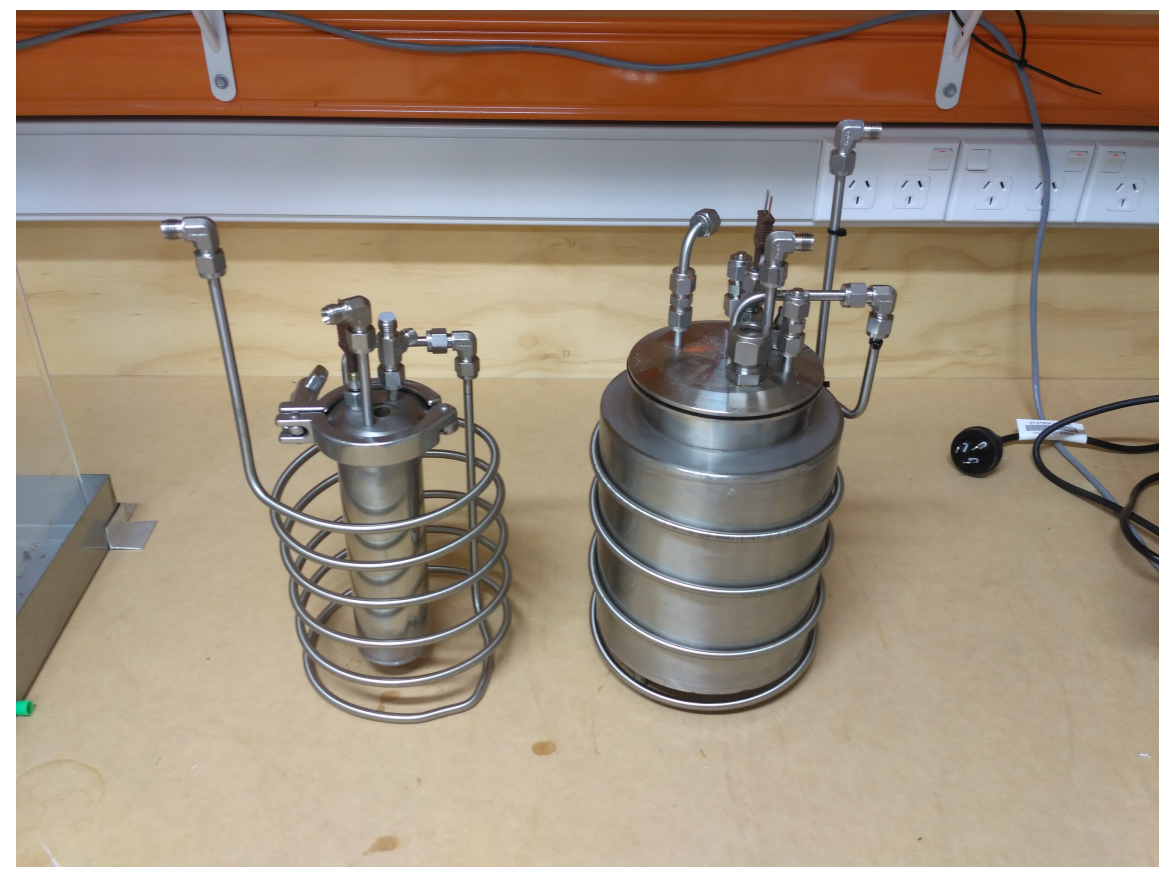

Figure 3.5: The 0.5 litre (left) and the 3 litre (right) test chambers.

filled with nitrogen up to $p_{\mathrm{m} \_}=900 \mathrm{mbar}$ and then filled from a $5 \%$ hydrogen cylinder up to $p_{\mathrm{m} \_\max }=1125$ mbar creating a 1:5 (1\% hydrogen) mixture.

The hydrogen concentration $x_{\mathrm{H} 2}$ in the system after connecting the mixing chamber with the evacuated test chamber can be calculated using basic thermodynamics. The resulting equation is

$$
x_{\mathrm{H} 2}=\frac{\left.\left(p_{\mathrm{m} \_\mathrm{v}} V_{\mathrm{m}} x_{\mathrm{H} 2^{\prime}}+\left(p_{\mathrm{m} \_ \text {max }}-p_{\mathrm{m} \_n}-p_{\mathrm{m}_{-} \mathrm{v}}\right)\right) V_{\mathrm{m}} x_{\mathrm{c}}\right) T_{\mathrm{t}}+\left(p_{\mathrm{t}_{\mathrm{v}} \mathrm{v}} V_{\mathrm{t}} x_{\mathrm{H} 2^{\prime}}\right) T_{\mathrm{m}}}{\left(p_{\mathrm{m} \_ \text {max }} V_{\mathrm{m}} T_{\mathrm{t}}+p_{\mathrm{t} \mathrm{v}} V_{\mathrm{t}} T_{\mathrm{m}}\right)}
$$

where $x_{\mathrm{H} 2^{\prime}}$ is the hydrogen concentration of the residual gas in the system before the change of gases, $x_{\mathrm{c}}$ is the hydrogen concentration of the cylinder, $V$ and $T$ are the volume and temperature of the mixing chamber $(\mathrm{m})$ and of the test chamber $(\mathrm{t})$.

With equation (3.1) the concentration inaccuracy can be calculated as well. The average achieved concentrations and possible uncertainty for a step wise increase starting from $100 \mathrm{ppm}$ to 50,000 ppm are given in Table 3.3. The uncertainty is derived from the assumption that each pressure $p_{\mathrm{m}_{-\mathrm{v}}}, p_{\mathrm{m} \_\max }, p_{\mathrm{m} \_\mathrm{n}}$, and $p_{\mathrm{t}_{-\mathrm{v}}}$ itself has a \pm 2 mbar uncertainty. The values were calculated with cylinder 1 for concentrations of 2500 ppm and below and with cylinder 2 for the remaining concentration (compare Table 3.2). In general, the relative deviation from the desired hydrogen concentration is lower than $5 \%$ for concentrations up to 5 times lower than that of the original cylinder.

The temperature and pressure inside the test chamber were recorded using a T-type thermocouple and a pressure transducer (PX2EN1XX050PAAAX, 


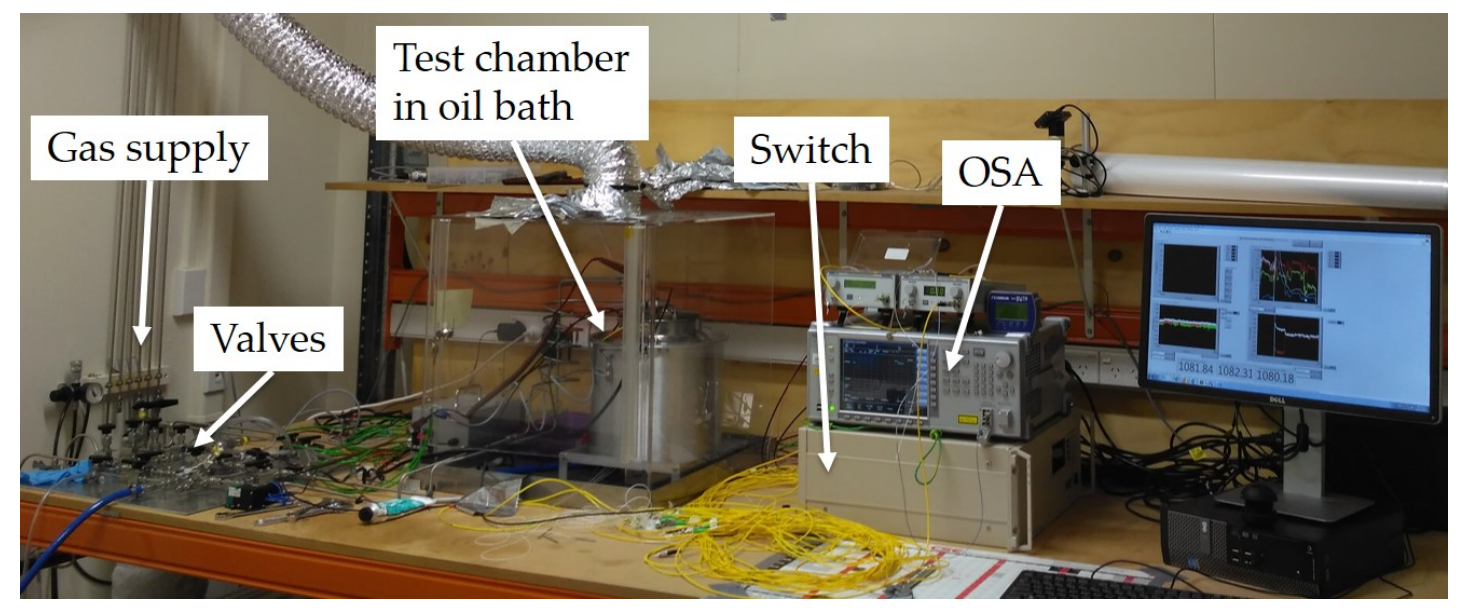

Figure 3.6: Test setup I with optical spectrum analyzer, optical switch, and computer.

Table 3.3: Accuracy of gas mixing. Expected (ppm) hydrogen concentration, Average (ppm) hydrogen concentration, Uncertainty ( \pm ppm) due to mixing inaccuracy

\begin{tabular}{rrrrrrrrrrr}
\hline Exp & 100 & 500 & 1,000 & 2,500 & 5,000 & 10,000 & 20,000 & 30,000 & 40,000 & 50,000 \\
\hline Ave & 100 & 501 & 1,005 & 2,467 & 4,994 & 10,048 & 20,096 & 30,198 & 40,299 & 49,355 \\
Unc & 17 & 21 & 25 & 36 & 329 & 350 & 387 & 416 & 445 & 392 \\
\hline
\end{tabular}




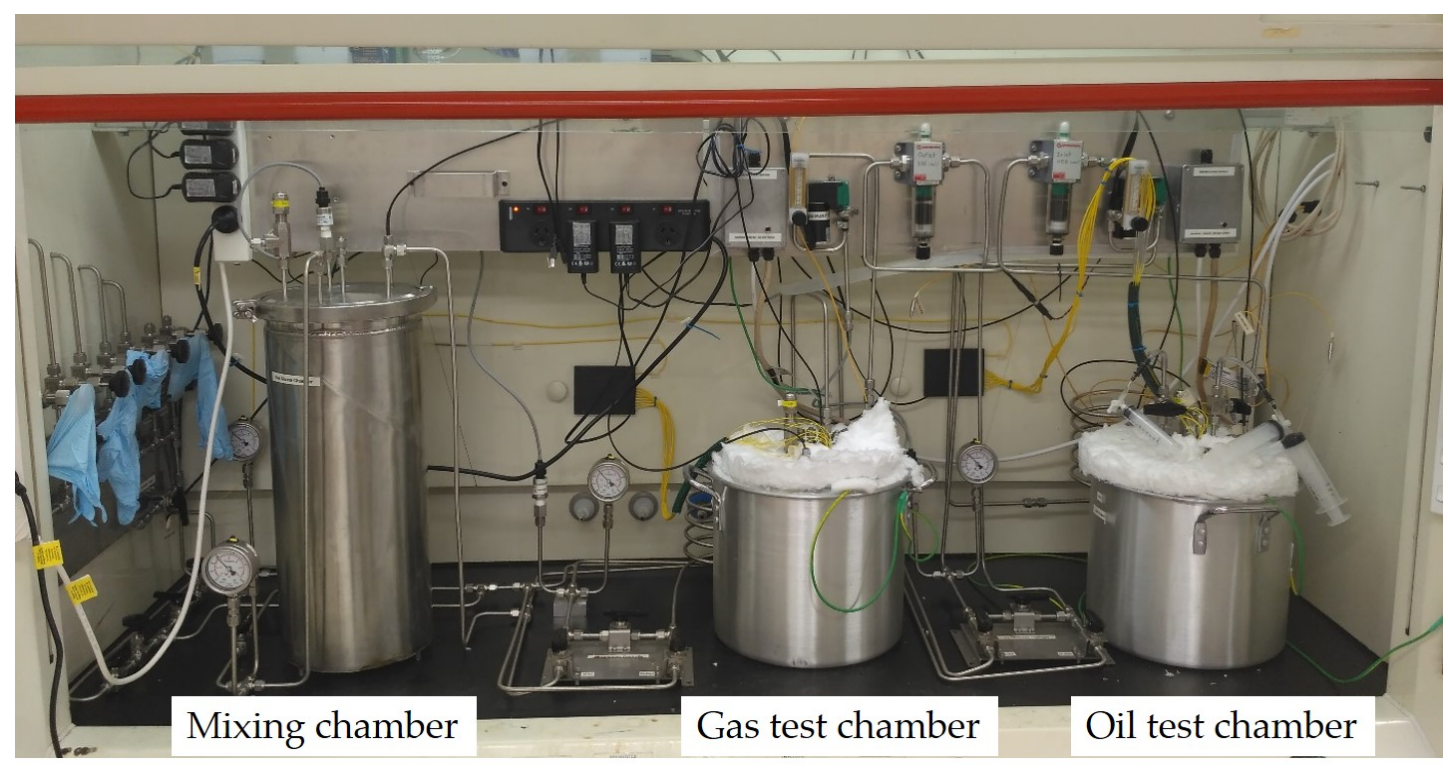

Figure 3.7: Test setup II with mixing chamber, gas test chamber and oil test chamber.

Honeywell; calibrated against a reference pressure transducer VD85, Thyracont), NI CompactRIO data acquisition and LabVIEW.

\subsubsection{Setup II for measurements in gas and oil}

The setup is based on the design of setup I. However, the setup has one mixing chamber (15 liter), one test chamber for gas and one for oil (each 3 litre)(see Figure 3.7). It can be either used for measurements in gas, or in oil. But it was not designed for simultaneous measuring in both test chambers. The operation of the setup is similar to setup I.

The oil test chamber has two ports to transfer oil: the oil sampling port, and the oil level port (see Figure 3.8 (a)). From the oil sampling port, oil can be extracted with syringes for dissolved gas analysis measurements. The inlet of the sampling port is located on the same level and close to the sensors. Over the oil level port, oil can be filled into and extracted from the test chamber with a syringe. This enables control of the oil level in the test chamber: If gas is sampled, the level is too low. If oil is sampled, the level is too high. If gas bubbles (in oil) are sampled the level is right.

A stainless steel pneumatic silencer $(\mathrm{G} 1 / 4$ thread) is used as a diffuser. The metal mesh creates small gas bubbles. Care was taken that the generated bubbles do not move to the sensor and influence the measurements, since the aim is to measure dissolved hydrogen and not hydrogen bubbles. To ensure that bubbles do not move to the surface an experiment was conducted: Oil 

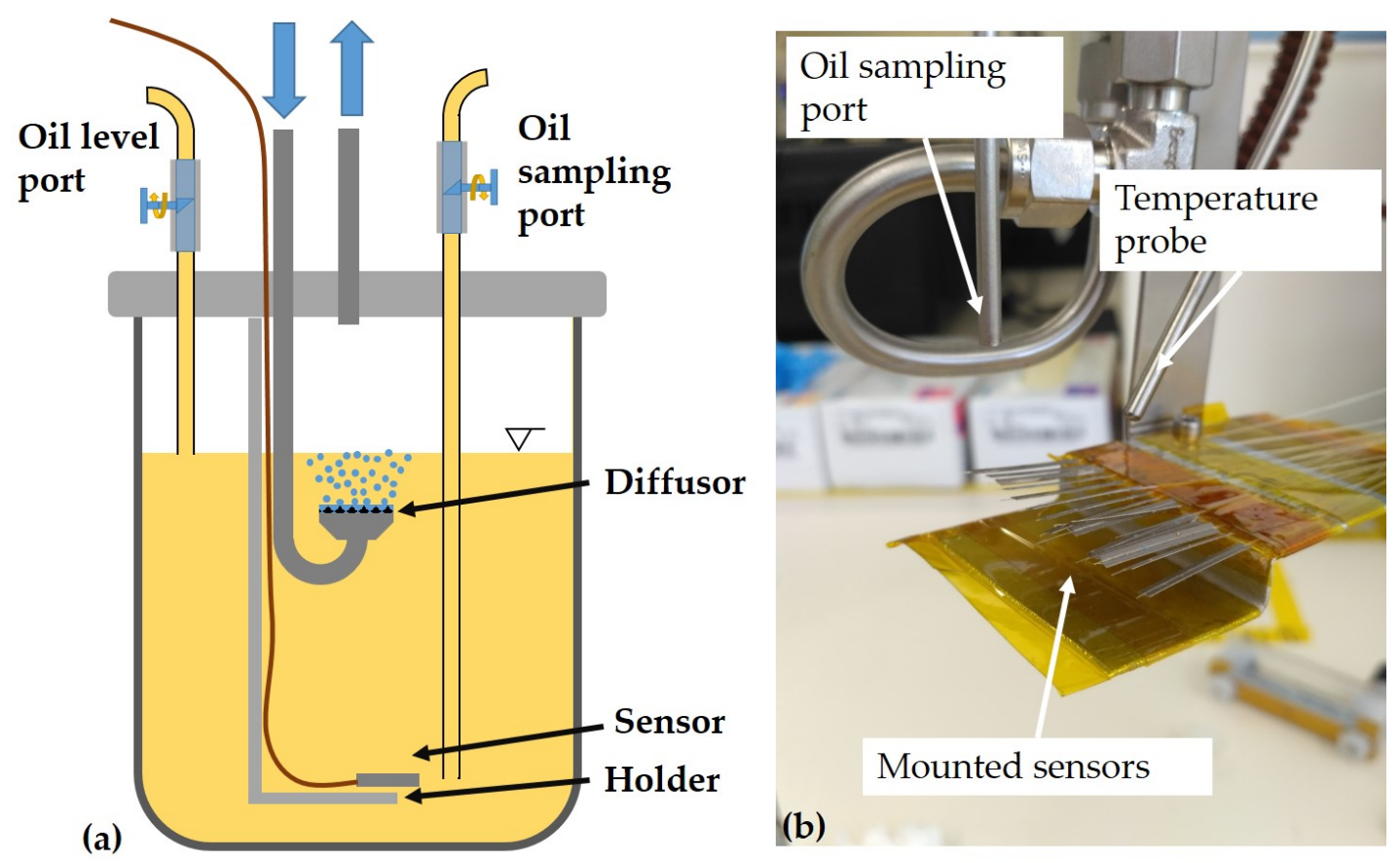

Figure 3.8: (a) schematic drawing of oil test chamber and (b) detail view of the mounted sensors, oil sampling port and temperature probe.

was placed in a glass beaker of similar dimensions to the test chamber. The diffuser was immersed in oil at varying depths and air bubbles were generated at different flow rates. For flow rates of $2-41 / \mathrm{min}$ and locations $40-60 \mathrm{~mm}$ underneath the oil level, no bubbles were observed to move below the diffuser level (Figure 3.9 (a)). Therefore, the diffuser is located $60 \mathrm{~mm}$ underneath he inlet of the oil level port and the flow rate was set between 2- $41 / \mathrm{min}$ for the experiments. Furthermore, no oil bubbles were observed in the samples during the experiments.

The hydrogen uptake in oil was determined by bubbling $5 \%$ hydrogen in nitrogen at $90^{\circ} \mathrm{C}$ and 1060 mbar. The results are shown in Figure 3.10. An exponential decay fit is applied to the data, which is the solution of a simplified 1-Dimensional diffusion model [112]. Using the parameter of the fit, the time to reach a certain value can be estimated. It takes 4.7 hours to reach $99 \%$ and 7.1 hours to reach $99.9 \%$ of the value of the final concentration. The final concentration was measured at 4322 ppm which leads to an Ostwald coefficient (solubility, see Section 2.1.5) of hydrogen of 0.086 for this particular mineral oil at $90^{\circ} \mathrm{C}$ and 1060 mbar.

The temperature and pressure inside the test chambers were recorded using a Pt100 (in gas), or a T-type thermocouple (in oil) and pressure transducers (PX2EN1XX050PAAAX, Honeywell) calibrated against a reference pressure transducer (VD85, Thyracont), Pico technology, or NI CompactRIO data acqui- 


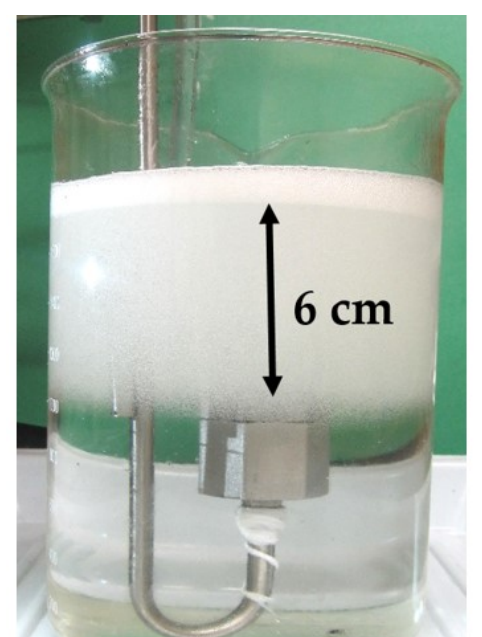

(a)

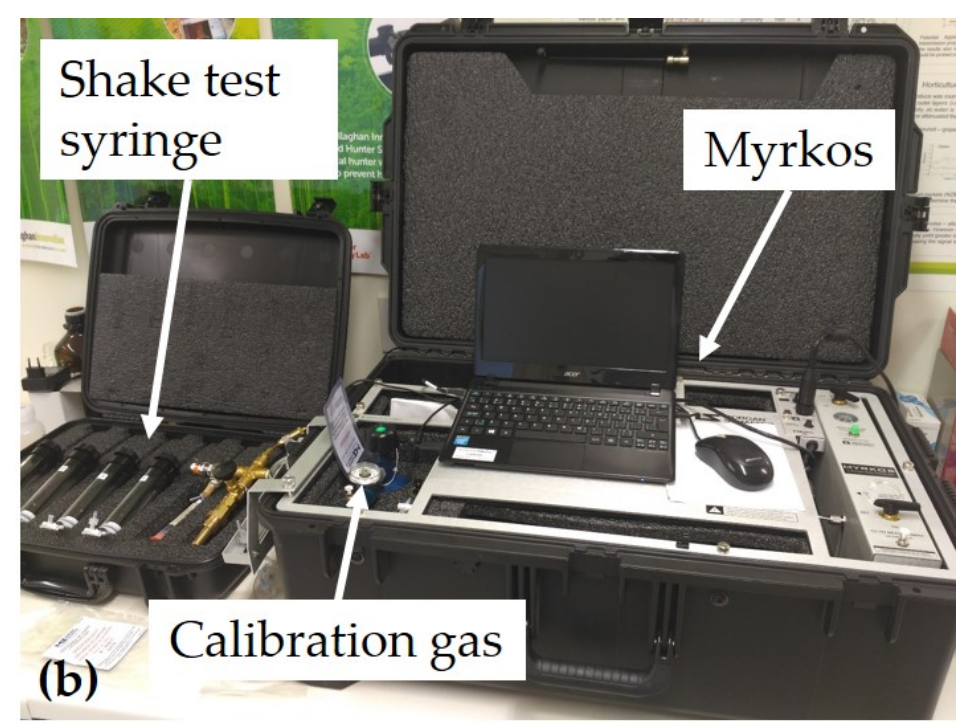

Figure 3.9: (a) Bubbling experiment of air in oil at room temperature. (b) Myrkos field package.

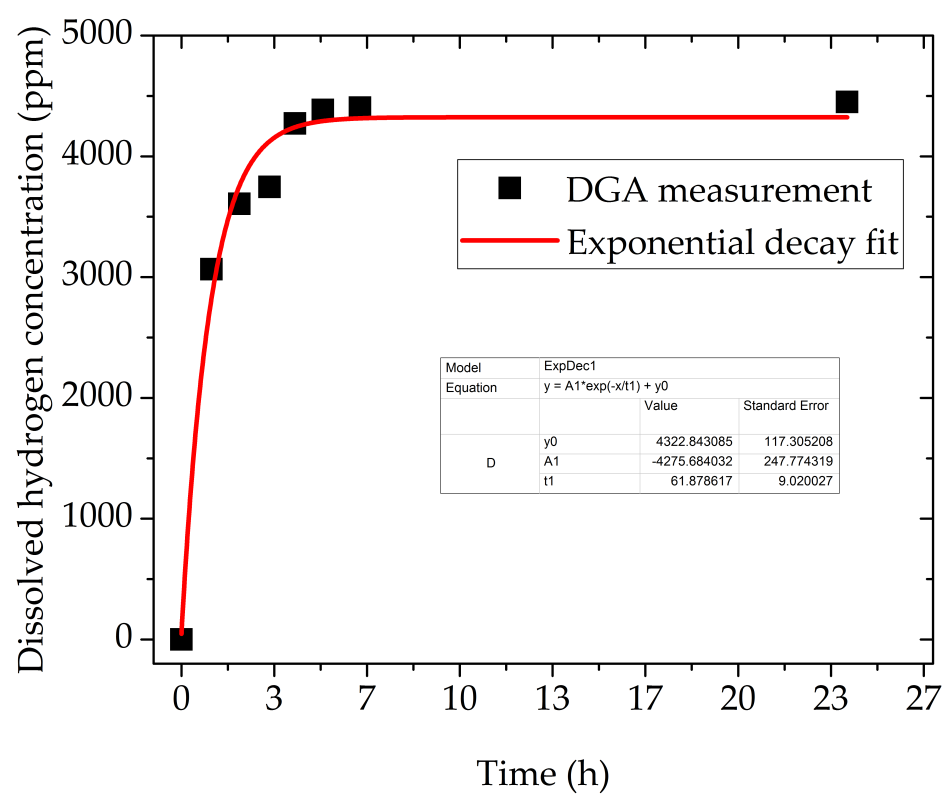

Figure 3.10: Hydrogen uptake in transformer oil. 
sition and LabVIEW.

\subsubsection{Gas chromatograph}

To measure the dissolved gases in the transformer oil, a special gas chromatograph is used (see Figure 3.9 (b)). The commercial DGA system (Myrkos Version: Field package, Morgan Schaffer) is a mobile gas chromatograph that uses the Morgan Shaffer Shake Test to extract the gases from the oil. Helium is used as a carrier gas and needs to be refilled regularly. The gas chromatograph is calibrated each day of use with calibration gas according to a standard procedure. After calibration, oil samples can be analysed.

The oil samples are taken with Shake Test syringes over the sampling port. When sampling, the oil is at $90^{\circ} \mathrm{C}$. It has been found to be useful (Not handling hot oil, improved repeatability) to let the oil cool down in the syringes before proceeding with the analysis. The Shake Test and the analysis is performed according to the Morgan Schaffer instructions. A software based instruction guides the user through all steps of the process, from sampling the oil, extracting the gas with the Shake Test, to analysing it in the Myrkos. This makes the process highly repeatable. The Myrkos has a linear measurement range for dissolved hydrogen concentration from 5- 700 ppm. To analyse higher concentrations, the oil samples need to be diluted according to the instructions. We found that there is a systematic error in how the dissolved hydrogen concentrations are determined in the Myrkos depending on the dilution of the sample (see Section 8.2.1.

\subsection{Tensile test}

To verify the hypothesis of correlating the strain transfer coefficient obtained by strain calibration to the strain transfer coefficient obtained by temperature calibration, a tensile test machine and tensile test bars were used. Both are described in this section.

A Micron Optics si155 unit and a DiCon GP700 optical switch was used to interrogate the fiber. The temperature was measured using a Pt100 probe. The Pt100 and the resistive strain gauges were read-out using a National Instruments CompactDAQ with an NI9219 module. All measurements were synchronized using LabVIEW. 

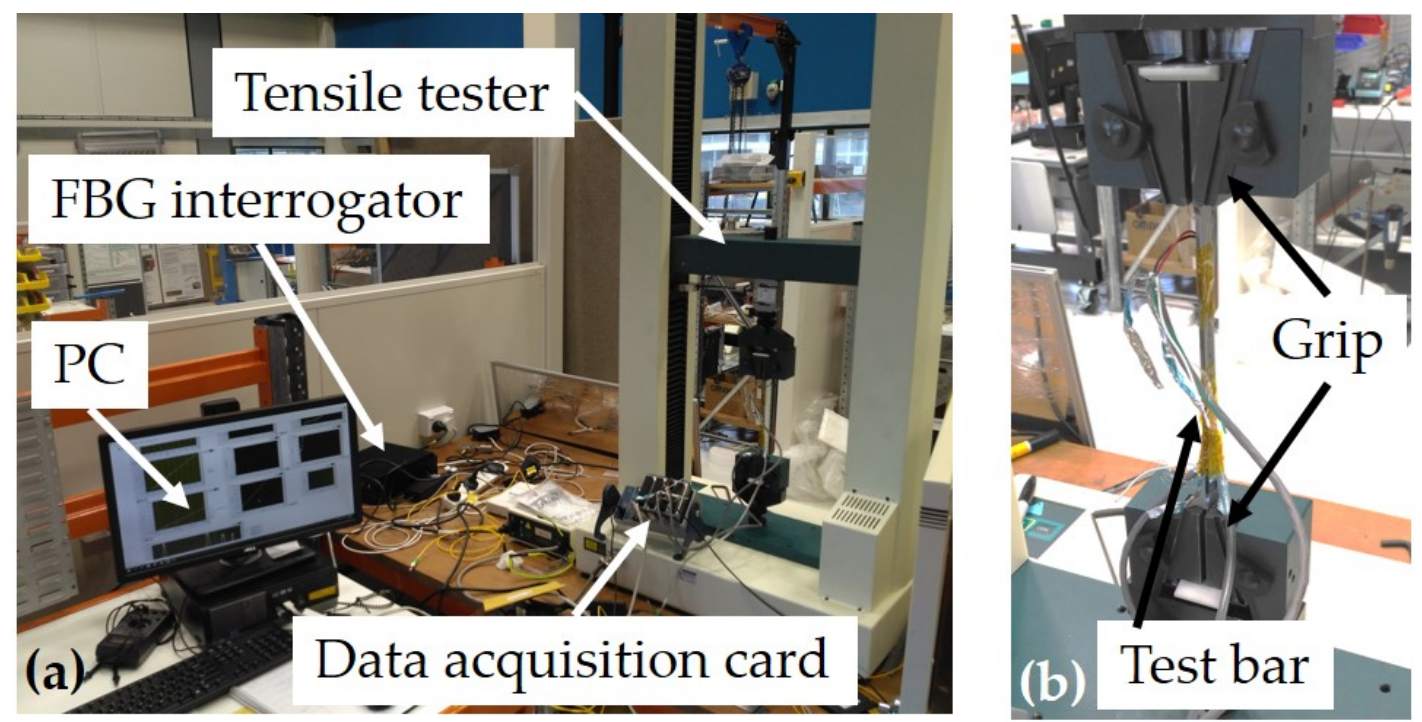

Figure 3.11: (a) The tensile test setup and (b) close-up of the wedge grips with loaded test bar.

\subsubsection{Tensile test machine - Tinius Olsen H10KT}

The Tinius Olsen H10KT is a uni-axial tensile test machine that allows loads up to $10 \mathrm{kN}$ (see Figure 3.11 (a)). The machine can be controlled via a computer and LabVIEW. The tensile test bars are clamped in wedge grips (see Figure $3.11(b))$.

\subsubsection{Tensile test bar}

Six tensile test bars of mild steel were manufactured according to ASTM E8 (see Figure 3.12 (a)) [12]. On the front of each bar, two resistive strain gauges, one uni-axial gauge (BX120-3AA) and one biaxial gauge (BX120-2BB) were adhesively bonded. The uni-axial gauge was used to determine the Young's modulus of the material, to allow calibration of the biaxial gauge. The inherently temperature compensated biaxial gauges were used to determine the strain of the test bar. On the back of each bar, three FBGs were adhesively bonded. Each of the six bars had a different arrangement of the FBGs. To vary the coefficient of strain transfer of the FBGs, a set of different coating materials and different bond lengths were used (see Figure 3.12 (b)). The commercial FBGs have either an acrylate recoating, a Ormocer $\mathrm{T}$ coating (draw tower grating), or are bare (stripped) FBGs. The bond length of the Ormocer T coated FBGs and the bare FBGs was $20 \mathrm{~mm}$. Bond lengths of 20,40,60, and $80 \mathrm{~mm}$ were employed for the acrylate coated FBGs. The bond length was defined by masking the test bar with two Kapton tape pieces (see Figure 3.12 (a)). A cyanoacrylate adhe- 


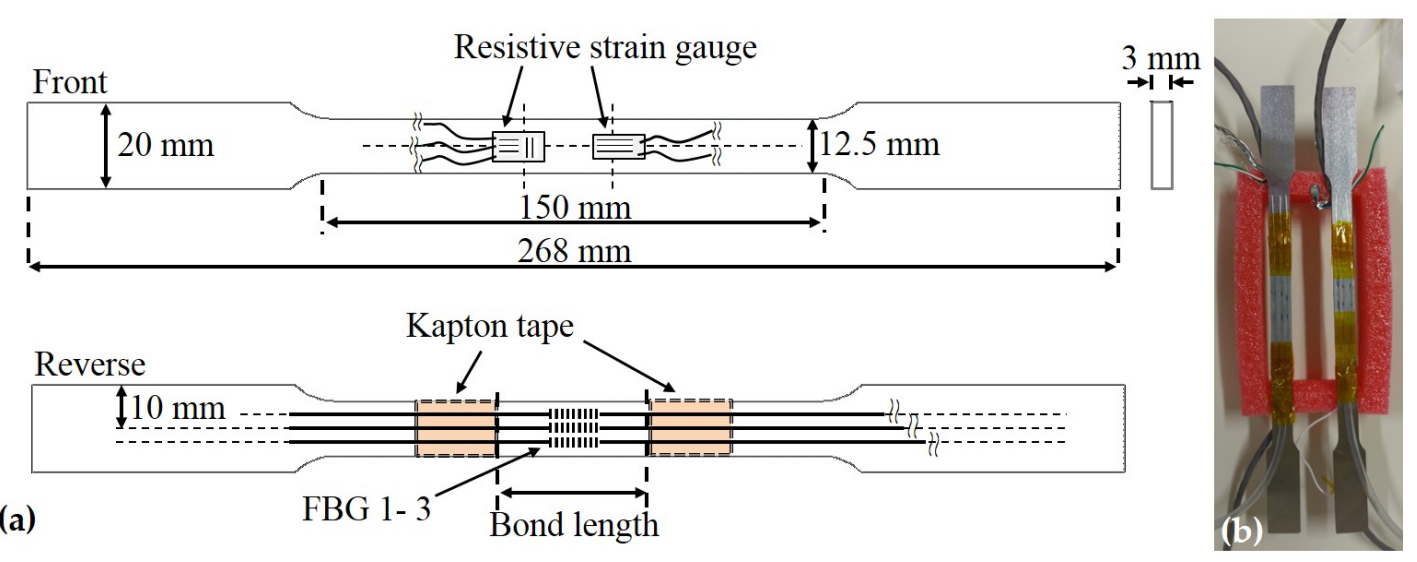

Figure 3.12: (a) schematic of tensile test bar front and reverse. (b) photograph of two tensile test bars with different bond length.

sive (Loctite 401) was used for bonding both the resistive strain gauges and FBGs. The adhesive was cured for 24 hours at room temperature. 


\section{Chapter 4}

\section{Sensor design}

This chapter describes the main criteria and methodology to design an optimized palladium based hydrogen sensor. First, the sensor optimization parameters (a) selection of the working temperature of the sensor to avoid a phase change, (b) selection of the palladium geometry to achieve the required sensitivity, and (c) amplification of the signal to increase the sensitivity are explained. Second, the manufacturing processes of the different sensor designs are described. The processes described are in chronological order. First coated sensors were manufactured. To improve the sensitivity a wrapped $20 \mu \mathrm{m}$ and a $100 \mu \mathrm{m}$ palladium foil sensors were designed and manufactured. For both the initial manufacturing processes were improved. The improvement in manufacturing also led to another new design of a sandwiched $20 \mu \mathrm{m}$ palladium foil sensor. In addition, two concepts with increased sensitivity through amplification have been developed. One is a sandwiched $10 \mu \mathrm{m}$ palladium silver foil sensor with increased hydrogen solubility and the other one relies on a new, original pre-strained sensor concept. Finally, different hydrogen calibration methods for the sensors are outlined.

\subsection{Sensor parameter optimization}

In this section we discuss the different parameters that can be optimized when designing a hydrogen sensor. First, the selection of the working temperature is described, than the influence of the palladium geometry is discussed, and finally two amplification techniques are described.

\subsubsection{Selecting the working temperature to avoid phase change}

In this section the selection of the working temperature of the sensor is described. This is crucial to avoid the phase change region which can have sev- 


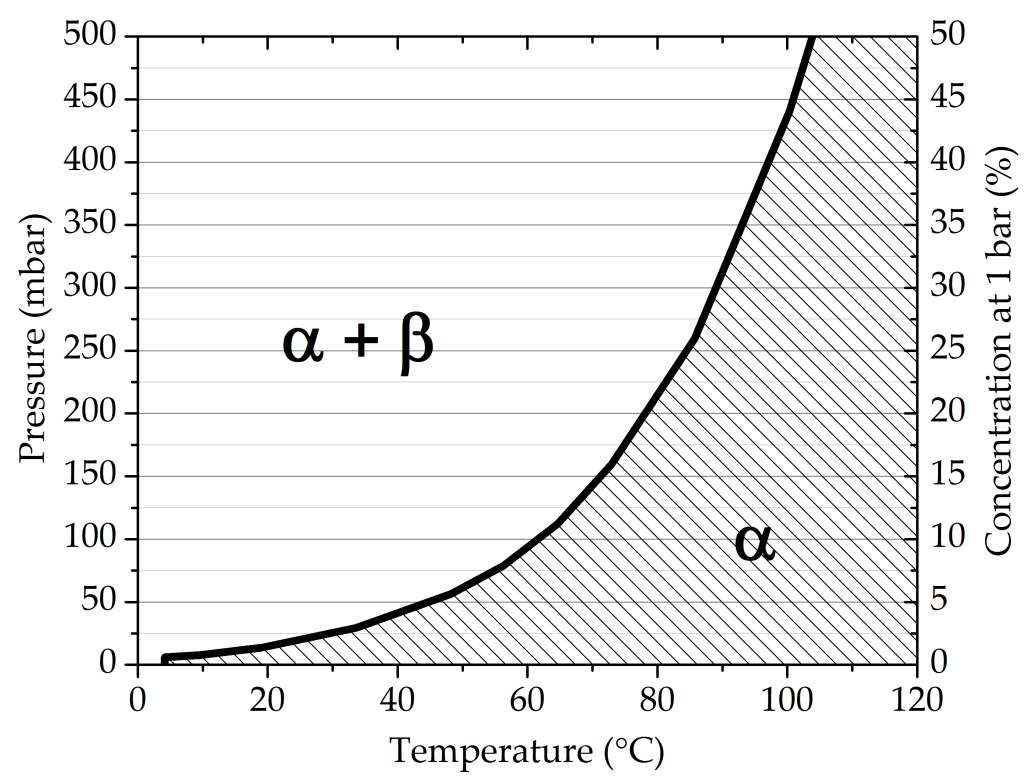

Figure 4.1: Modified phase diagram of palladium hydride with hydrogen partial pressure/ concentration vs. temperature (derived from [21]).

eral negative effects on the sensor (see Section 2.2.4). Predominantly, the extensive expansion of the palladium due to the $\beta$-phase can cause an irreversible, plastic deformation of the palladium, and the resulting hysteresis decreases the accuracy of the sensor.

Therefore, when designing a reliable palladium based hydrogen sensor the phase change region must be avoided. This can be achieved by selecting an appropriate working temperature so the expected hydrogen concentration does not lead to a phase change, i.e., $n$ does not exceed $\alpha_{\max }$. Simons and Flanagan [126] present $\alpha_{\max }$ as a spinodal curve showing $n$ versus temperature. For sensor design purposes, this diagram can be improved by plotting the spinodal curve with hydrogen partial pressure (or concentration calculated as $\left.c=p_{\text {partial }} / p_{\text {total }}\right)$, versus temperature. The partial pressure is calculated with equation 2.11 for given $n$ and temperature. This leads to a new diagram with extended information content and improved usability (see Figure 4.1). Now, temperature and hydrogen partial pressure, or concentration can be directly correlated to the phase change, without an additional step of correlating $n$ to the hydrogen partial pressure, or concentration.

Figure 4.1 can be utilised as follows: In the region below the spinodal curve the palladium hydride is in the $\alpha$-phase; in the region above the curve the miscibility gap (both phases are present see Section 2.2.4) is entered. Selecting the working temperature is now easy and intuitive: The minimum working 


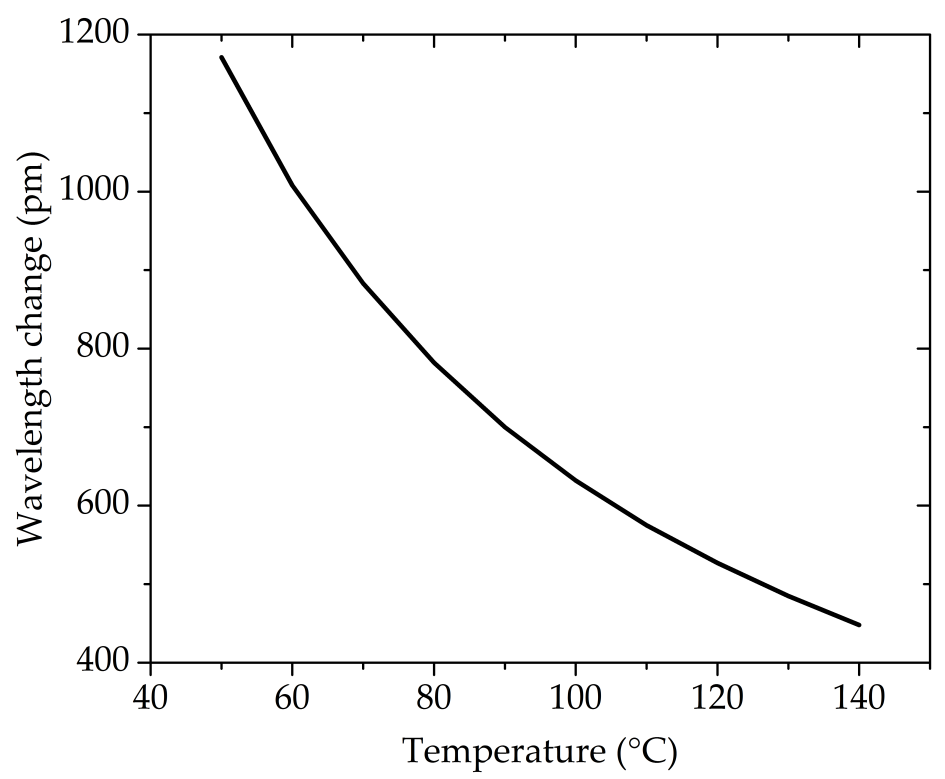

Figure 4.2: Temperature dependency of hydrogen sensitivity. Calculated maximum response (wavelength change) for 5\% hydrogen at 1 bar and various temperatures.

temperature is the abscissa corresponding to the intercept of the maximum expected hydrogen partial pressure, or concentration with the spinodal curve. For example, if we want to sense up to $5 \%$ hydrogen at 1 bar (50 mbar partial pressure) we have to set the working temperature of the sensor to be at least $50^{\circ} \mathrm{C}$ to avoid a phase change. Conversely, the selection of a working temperature can be used to determine the maximum hydrogen concentration to which the sensor can be exposed before entering the phase change region. For the purposes of the investigation reported here, the measurements were conducted at $30^{\circ} \mathrm{C}, 60^{\circ} \mathrm{C}, 90^{\circ} \mathrm{C}$, and $120^{\circ} \mathrm{C}$ leading to a maximum hydrogen partial pressure of 25, 100,300, and 800 mbar respectively before entering the phase change region. Therefore, the hydrogen partial pressure for each experiment was chosen below those values.

The working temperature should be selected to avoid the phase change region, but not significantly higher, since increasing the working temperature decreases the sensitivity (see Figure 4.2). 


\subsubsection{Selecting the palladium geometry to achieve the required sensitivity}

Apart from the working temperature, the geometry of the palladium itself has a large impact on the hydrogen sensitivity of the sensor. In this section, the selection of a palladium geometry to achieve a certain sensitivity is described. First, the maximum possible sensitivity of the sensor is calculated, neglecting the influence of (a) the strain sensor itself limiting the expansion of the palladium, and (b) imperfect strain transfer between palladium and sensor. Both of these factors influence the sensitivity negatively. Using (2.11) we can calculate $n$ for a given hydrogen partial pressure and temperature. Using (4.1) we can calculate the expansion $\varepsilon_{\mathrm{Pd} \_\mathrm{H} 2}$ of palladium due to absorbed hydrogen [6].

$$
\varepsilon_{\mathrm{Pd} \_\mathrm{H} 2}=\frac{\Delta l}{l}=C n
$$

where $C$ is calculated using (4.2) and (2.12) to be $0.063 \pm 0.003$ [6]:

$$
\frac{\Delta l}{l}=\frac{1}{3} \frac{\Delta V}{V}=\frac{1}{3}(0.19 \pm 0.01) n
$$

The expansion of the palladium is transferred to a strain sensor. Therefore, the sensitivity of the hydrogen sensor depends on the sensitivity of the strain sensor used. In this thesis, FBGs were used as strain sensors. The strain sensitivity of an FBG (silica-based, single-mode, 1550nm center wavelength) is 1.21 $\mathrm{pm} / \mu \varepsilon\left(\mu \varepsilon=10^{-6} \Delta l / l\right)[64]$.

We can now calculate the maximum response of an FBG for a given hydrogen partial pressure using (2.11),(4.1) and (2.15) and the assumption that $\Delta \varepsilon=\varepsilon_{\text {Pd_H2 }}$ (where $\Delta \varepsilon$ is the strain of the FBG itself). The result at a working temperature of $90^{\circ} \mathrm{C}$ is displayed in Figure 4.3.

However, $\Delta \varepsilon \neq \varepsilon_{\mathrm{Pd} \mathrm{H}_{2}}$ due to (a) the influence of the fiber itself forming a composite with the expanding palladium [27] and (b) the imperfect strain transfer between palladium and fiber. If we assume perfect strain transfer between palladium and fiber, where the strain of the FBG $\Delta \varepsilon^{\prime}$ just depends on $\varepsilon_{\mathrm{Pd} H 2}$ and the cross sectional area and Young's modulus of fiber and palladium, then the first (a) of these effects can be expressed as [27]:

$$
\Delta \varepsilon^{\prime}=\varepsilon_{\text {Pd_H2 }} \frac{Y}{1+Y}
$$

where, $Y=\left(A_{\mathrm{Pd}} E_{\mathrm{Pd}}\right) /\left(A_{\mathrm{f}} E_{\mathrm{f}}\right)$ and $A_{\mathrm{Pd}}$ and $A_{\mathrm{f}}$ are the cross sectional area (CSA) of the palladium $(\mathrm{Pd})$ and the fiber (f) respectivly, and $E_{\mathrm{Pd}}$ and $E_{\mathrm{f}}$ are the corresponding Young's modulus. The Youngs modulus of the bare fiber is $E_{\mathrm{f}}=69$ GPa [11] and of palladium is $E_{\mathrm{Pd}}=121 \mathrm{GPa}$ [59]. We can adjust $Y$, and thus the 


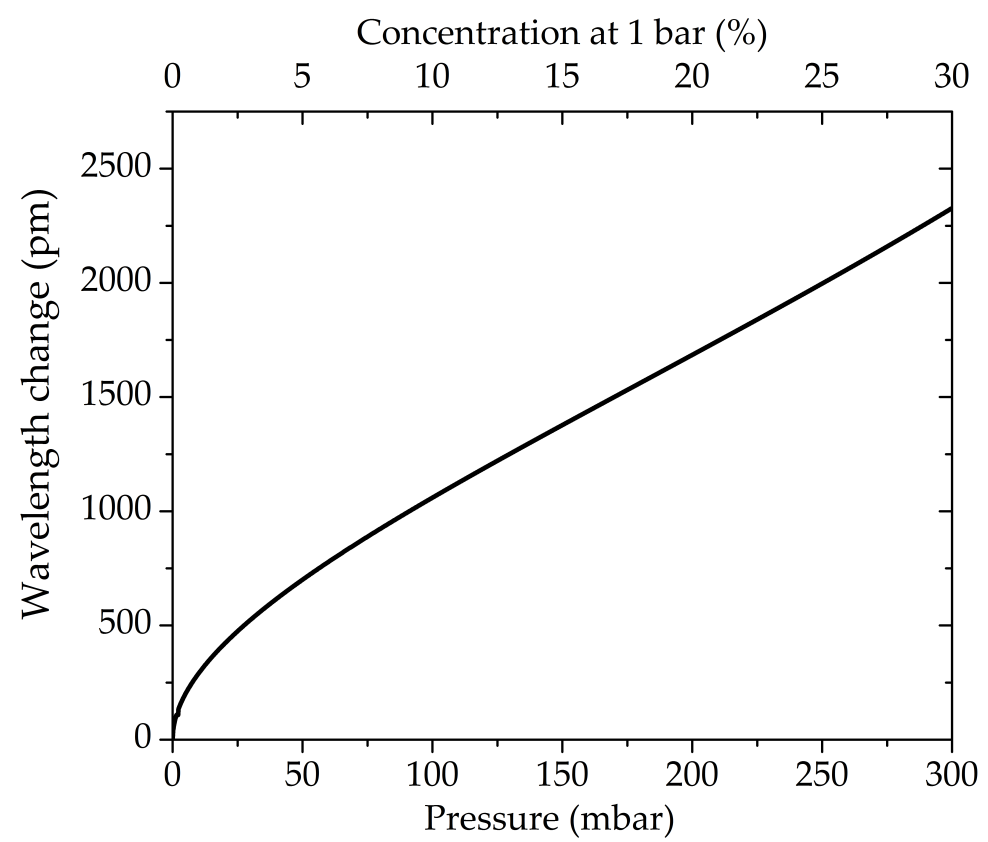

Figure 4.3: Maximum response (wavelength change) of a palladium based hydrogen sensor using an $\mathrm{FBG}$ at $90^{\circ} \mathrm{C}$.

hydrogen sensitivity of the sensor, by altering the ratio of the CSAs of palladium and fiber $\left(\mathrm{CSA}\right.$ ratio $\left.=\mathrm{CSA}_{\mathrm{Pd}} / \mathrm{CSA}_{\mathrm{f}}\right)$. The normalized wavelength change can be calculated using $Y /(1+Y)$ for different CSA ratios and is shown in Figure 4.4 .

The hydrogen response of the sensor can now be calculated by multiplying the calculated maximum wavelength change at a certain temperature (Figure 4.3) with the normalized wavelength change of a sensor having a certain CSA ratio (Figure 4.4).

\subsubsection{Amplification of the signal to increase the sensitivity}

If the required sensitivity cannot be achieved by selecting the right working temperature and geometry, two different options for amplifying the sensitivity of palladium are possible: choosing a sensor design that mechanically amplifies the expansion for the palladium, or choosing a palladium alloy with increased hydrogen solubility.

Mechanical amplification is acheived by concentrating the expansion of a large palladium element on a short FBG. To our knowledge, only one example of the mechanical amplification has been reported for hydrogen sensing so far [95]. Figure 4.5 shows the working principle in a schematic drawing. The expansion of the palladium body with hydrogen absorption is transduced only in the short length of the FBG, since the sensor element itself is significantly 


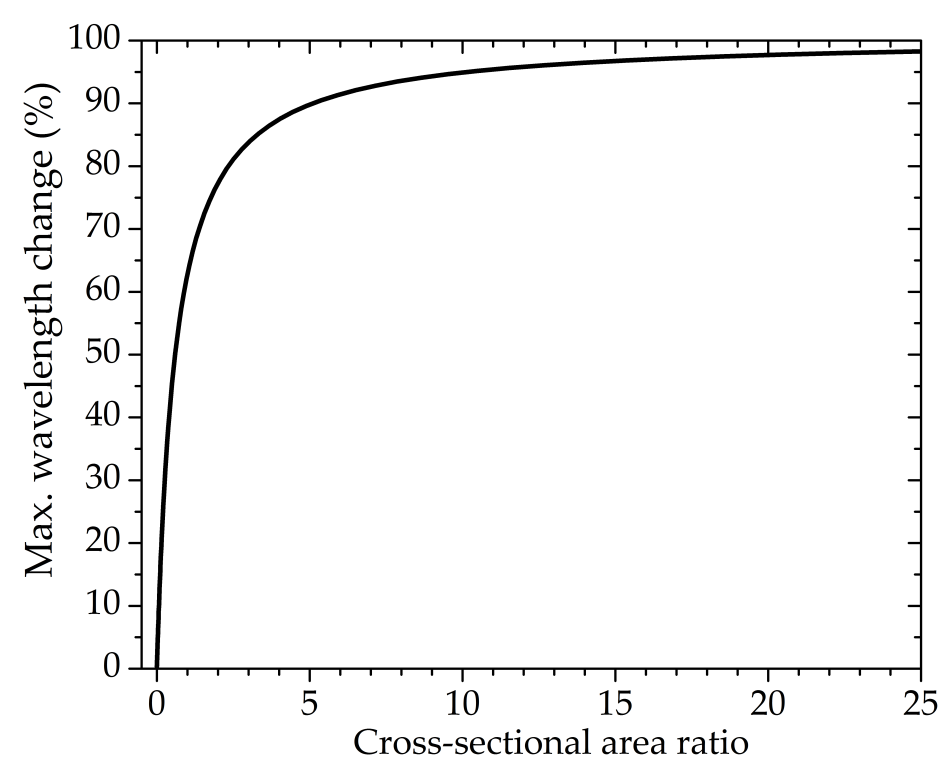

Figure 4.4: Influence of the ratio of cross-sectional area of palladium and fiber on the response of the sensor.

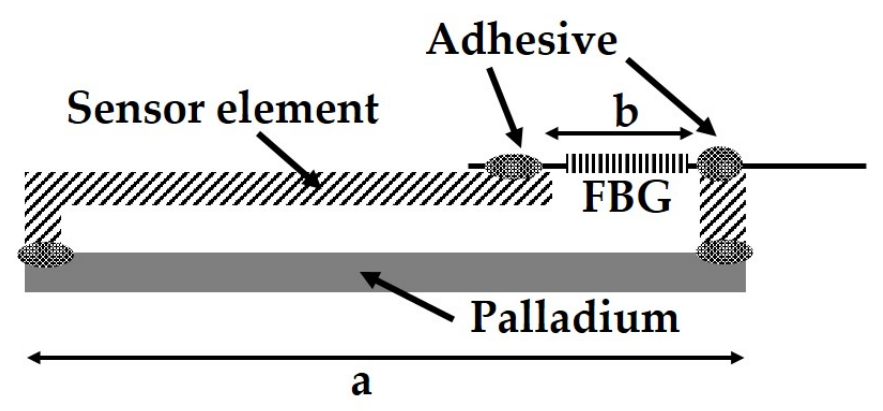

Figure 4.5: Strain concentration concept (adapted from [95]).

stiffer and does not expand. Here, the amplification is given by the ratio of the length of the palladium element $(a)$ to the length of the FBG $(b)$. For structural integrity of this sensor a relativly large and thick palladium tube was used (5 mm diameter, $300 \mu \mathrm{m}$ wall thickness) [95]. For temperature measurements with FBG several similar concepts have been reported [73, 83, 84, 84]

An example for amplification via increased hydrogen solubility is the use of palladium silver. Palladium silver has an increased hydrogen solubility at low $n\left(n<0.25, \mathrm{Ag}<30 \%, 50^{\circ} \mathrm{C}\right)$ [25]. This means higher $n$ at constant temperature and hydrogen partial pressure than pure palladium [82]. The Sieverts's constant (see Table 2.2) can be used to estimate the increase of hydrogen solubility and therefore amplification of the hydrogen sensitivity of palladium silver compared to pure palladium. Using Sieverts's law (2.10) the amplifica- 
tion is can be expressed as

$$
\varphi=\frac{\varepsilon_{\mathrm{PdAg}}}{\varepsilon_{\mathrm{Pd}}}=\frac{n_{\mathrm{PdAg}}}{n_{\mathrm{Pd}}}=\frac{K_{\mathrm{PdAg}}}{K_{\mathrm{Pd}}}
$$

where $\varepsilon$ is the strain of the metal due to hydrogen absorption, $n=\mathrm{H} / \mathrm{Pd}$ is hydrogen concentration in the palladium and $K$ is the Sievertss constant. By alloying 10, 20, and 30\% silver an amplification of 4.2, 9.0, and 17.2 can be expected at $90^{\circ} \mathrm{C}$. Several coated FBG sensors relying on this amplification concept have been reported (see Table 2.3).

\subsection{Sensor design and manufacturing}

After explaining the main design principles for an optimized hydrogen sensor, they are applied.In this section the design and manufacturing processes of the different hydrogen sensors developed for this thesis are described. Several different sensor designs have been developed and manufactured: coated sensors and foil sensors. The coated sensors are all-around coated with palladium. The foil sensors have either a 20 or $100 \mu \mathrm{m}$ palladium foil attached to the fiber. Furthermore, two sensors with amplified sensitivity were developed. One uses palladium silver foil with a higher hydrogen solubility and the other one uses a new concept based on a pre-strained sensor holder. For the wrapped $20 \mu \mathrm{m}$ (see Section 4.2.2) and $100 \mu \mathrm{m}$ (see Section 4.2.3) palladium foil sensors two manufacturing processes are described. Each have an initial and improved manufacturing process. Furthermore, for the initial manufacturing process two adhesives $\mathrm{NOA} 83 \mathrm{H}$ and $\mathrm{NOA} 86 \mathrm{H}$ were used. As both have a glass transition temperature below $45^{\circ} \mathrm{C}$, the adhesive was changed to ADR 243 for the improved and the other manufacturing processes.

\subsubsection{Coated sensor}

To increase the sensitivity of the coated sensor the CSA ratio of the sensor is increased by decreasing the diameter of the FBG. The diameter was reduced from 125 to $85 \mu \mathrm{m}$ by etching the FBG with potassium hydroxide $35 \mathrm{wt} \% \mathrm{KOH}$ at $130^{\circ} \mathrm{C}$. Then the fiber was mounted on a fixture (see Figure $3.2(\mathrm{a})$ ), allowing sputter coating from two sides. A thin layer of titanium, chrome, nickel between palladium and fiber is often used to increase the adhesion and prevent the palladium from peeling off the fiber $[36,41,44,114,158]$. Therefore, a 30 $\mathrm{nm}$ titanium adhesion layer and $1600 \mathrm{~nm}$ palladium layer were sputter coated consecutively. Afterwards, the fixture was turned upside down and the other side of the fiber was coated (schematic cross-sectional view of the sensor in 

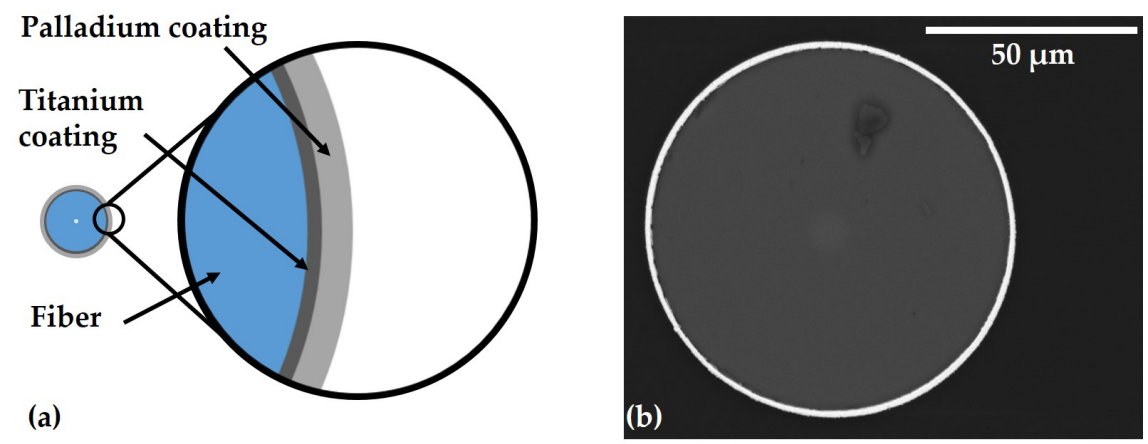

Figure 4.6: Cross-sectional view of coated sensor. (a) schematic, (b) SEM image.

Figure 4.6 (a)). SEM analysis of the sensors shows that sputtering creates an elliptically shaped coating around the etched fiber (Figure 4.6 (b)). The thickness on the thick side is around $1600 \mathrm{~nm}$ and at the thin side around $1200 \mathrm{~nm}$.

\subsubsection{Wrapped $20 \mu \mathrm{m}$ palladium foil sensor}

The first foil sensor produced was a $20 \mu \mathrm{m}$ palladium foil adhesively bonded to the FBG. The palladium foil creates a significantly larger CSA ratio compared to the coated sensor resulting in an increased hydrogen sensitivity of the sensor. Since the $20 \mu \mathrm{m}$ palladium foil can be easily shaped, a process was developed to create a thin bond line by wrapping the foil around the fiber. For this sensor two different manufacturing processes are described. The initial manufacturing process was developed for a proof of principle, is fully manual and therefore, the reproducibility is strongly dependent on the human factor. The improved manufacturing process was developed to increase the reproducibility of the sensor, included a manufacturing fixture and reduced the influence of the operator.

The initial manufacturing process is shown in Figure 4.7 (a). The process is as follows: Firstly, the fiber was coated with an adhesive (NOA83H). Secondly, the foil $(20 \mathrm{~mm} \times 10 \mathrm{~mm}$, the dimension was chosen to cover the whole length of the FBG and to allow wrapping, and tightening) was wrapped around the fiber. Then, with the help of two straight-edge tools, the foil was tightened around the fiber while laying on a hotplate. The hotplate was set to a surface temperature of $125^{\circ} \mathrm{C}$. The fiber-foil-assembly (still under tension) was cured on the hotplate for 10 minutes. Finally, the sensors were post cured in the experimental setup at $90^{\circ} \mathrm{C}$ for 12 hours. Figure 4.7 (b) shows that wrapping the $20 \mu \mathrm{m}$ palladium foil around the FBG creates a thin bond line on two opposing parts of the fiber, and two adhesive filled voids.

The initial manufacturing process described has the advantage that only 

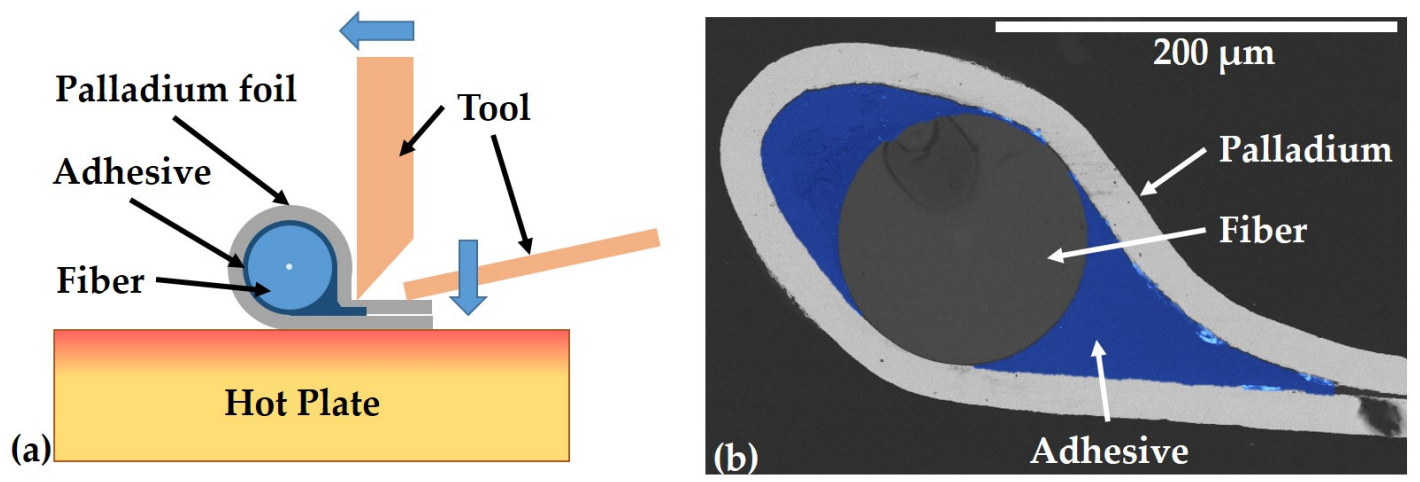

Figure 4.7: Cross-sectional view of $20 \mu \mathrm{m}$ foil sensor. (a) schematic manufacturing process, (b) SEM image (adhesive in false colour).

simple tools are necessary. However, the disadvantage is, that tightening the foil around a bare,fragile fiber and keeping the pressure on the foil-fiber-assembly constant during the 10 minute cure is challenging task for the operator.

The improved manufacturing process was developed to reduce the human factor and improve the reproducibility of the sensor. Thus, a manufacturing fixture was made that tightens thin palladium foils around a bare fiber through the use of a spring loaded stage (see Figure 4.8(a)). The fixture enables a repeatable force to be applied during manufacturing. The fixture consist of a clamp holding the palladium foil, a spring-loaded stage to strain the palladium foil, and two sheets to create a slot through which the palladium foil is pulled. All of these components are mounted on a metal angle. The manufacturing process is as follows (see Figure 4.8 (a)): Firstly, the $20 \mu \mathrm{m}$ palladium foil $(20 \times 20 \mathrm{~mm}$, the dimension was chosen to cover the whole length of the FBG and to allow clamping) is folded along the sides, leaving a loop to later insert the fiber. Secondly, the free ends are clamped down and the clamp is mounted on the stage. Then, the loop is guided through the slot, before the sheets are tightened leaving a narrow slot. The fiber is coated with adhesive (ADR243), inserted and placed within the loop. Afterwards, the stage is slowly released and the force of the spring pulls back the stage, and with it the foil. The size of the loop decreases until it sits firmly around the fiber. Finally, the whole assembly is placed on a hotplate with the foil-fiber facing the hotplate. The surface temperature of the hotplate is set to $100^{\circ} \mathrm{C}$ and the adhesive is cured for 3 hours. An SEM analysis showed similar results to the initial manual manufacturing method (Compare Figure 4.7(b) and Figure 4.8(b)). However, the loop created by the wrapping tool is larger, which leads to more palladium in the circumference of the fiber and is expected to have an increased hydrogen response. 


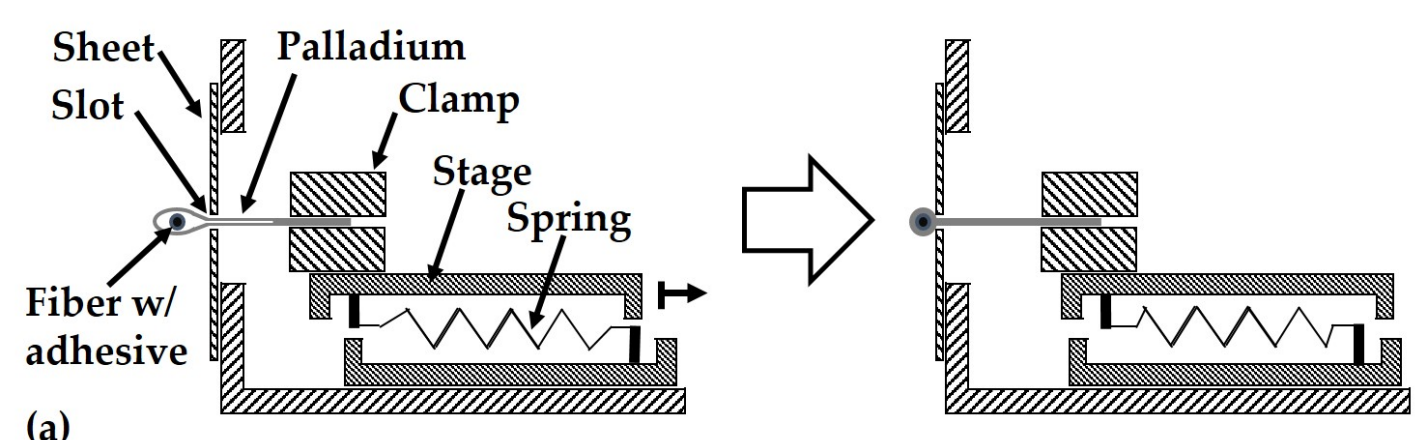

(b)

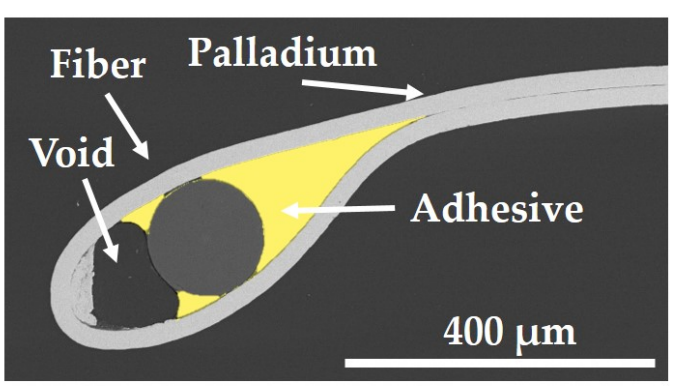

Figure 4.8: (a)Cross-sectional view of the foil wrapping fixture during the wrapping process. (b) SEM image of the $20 \mu \mathrm{m}$ palladium foil sensor manufactured with the foil wrapping tool (adhesive in false colour).

The advantage of the improved manufacturing process is that with the manufacturing fixture a constant force is applied on the foil-fiber-assembly during curing and reducing the human factor and increasing the repeatability.

\subsection{3 $100 \mu \mathrm{m}$ palladium foil sensor}

The second foil sensor has a $100 \mu \mathrm{m}$ palladium foil adhesively bonded to the FBG. The increased foil thickness compared to the $20 \mu \mathrm{m}$ palladium foil results in further improvement in sensitivity of the sensor. The $100 \mu \mathrm{m}$ foil cannot be easily shaped compared to the $20 \mu \mathrm{m}$ foil. Therefore, a foil forming tool was manufactured to imprint a groove (75 $\mu \mathrm{m}$ radius) into the palladium foil (20 $\mathrm{mm} \times 2 \mathrm{~mm}$, the length was chosen to cover the whole length of the FBG and the width is a given product parameter) to increase the effective adhesive bond area between the foil and the FBG.

The foil forming tool is made of tool steel and has a negative and positive mold which are aligned by four pins. Two springs automatically open the tool after the forming process. The negative mould is a rectangular groove $(2 \mathrm{~mm}$ width) with a spark eroded circular groove ( $350 \mu \mathrm{m}$ diameter) in its center. The 

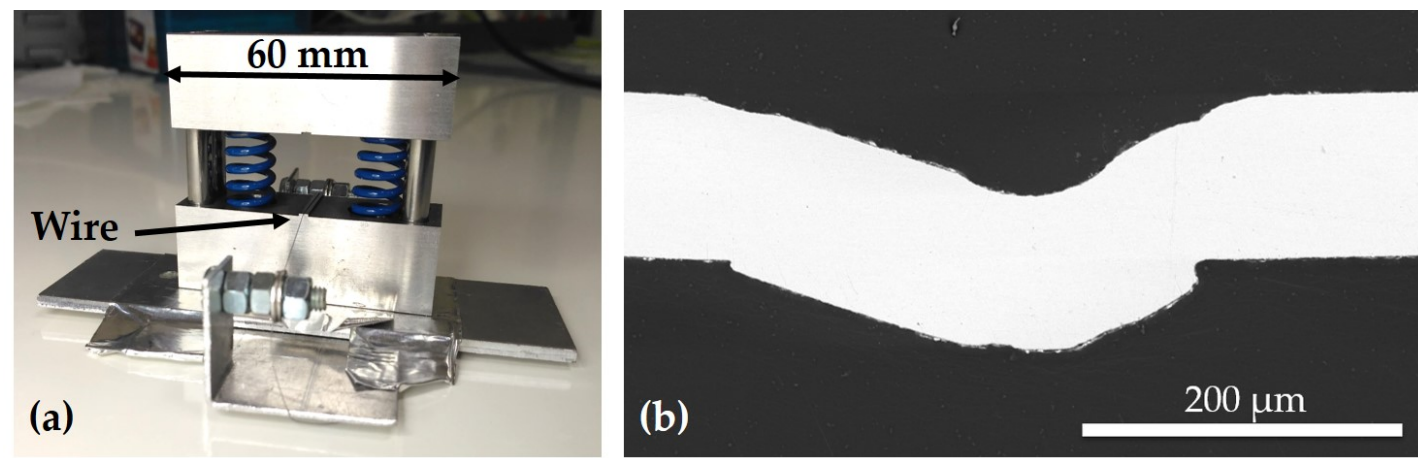

Figure 4.9: Foil forming tool (a) and SEM image of cross-sectional view of formed foil (b).

positive mould is a $150 \mu \mathrm{m}$ tungsten wire aligned in a V-groove. The wire is tightened and held in place with a tensioning device (see Figure 4.9 (a)). The tool was closed with a pneumatic press. With $1250 \mathrm{~N}$ closing force satisfactory results were achieved (see Figure 4.9 (b)).

For the $100 \mu \mathrm{m}$ palladium foil sensor also two different manufacturing processes are described. Similar to the $20 \mu \mathrm{m}$ palladium foil sensor presented in the previous subsection, the initial manufacturing process was developed for a proof of principle. It is also fully manual and therefore, the reproducibility can be strongly dependent on the human factor. For the improved manufacturing process the vacuum bagging method was adapted for our application. A manufacturing tool was developed, allowing the simultaneous manufacturing of up to 16 sensors.

The initial manufacturing process was as follows (see Figure 4.10 (a) and (b)). Firstly, the fiber is attached to the aluminium fixture in a way that the FBG touches the bottom under slight pressure, due to the bending of the fiber. Secondly, a small amount of adhesive is applied in the groove of the formed palladium foil and the fiber is coated with a thin adhesive layer $(\mathrm{NOA} 83 \mathrm{H}$, or $\mathrm{NOA} 86 \mathrm{H})$. Then, the fiber was positioned in the groove. A hotplate is used to cure the adhesive. The surface temperature of the hotplate is set to $125^{\circ} \mathrm{C}$ and the adhesive is cured for 10 minutes. Finally, the sensors are post cured in the experimental setup at $90^{\circ} \mathrm{C}$ for 12 hours. The initial manufacturing process has the advantage that only a few simple tools are necessary for manufacturing. The disadvantage is that the thickness of the adhesive layer is not well controlled. Analysing several sensors under the SEM showed that the thickness of the adhesive layer between fiber and palladium could range from around $d=1$ $\mu \mathrm{m}$ to $20 \mu \mathrm{m}$ (see 4.10 (c)and (d)).

The improved manufacturing process includes a purpose built tool that allows vacuum bagging of a palladium(foil)-fiber-composite (see Figure 4.11 (a)). 

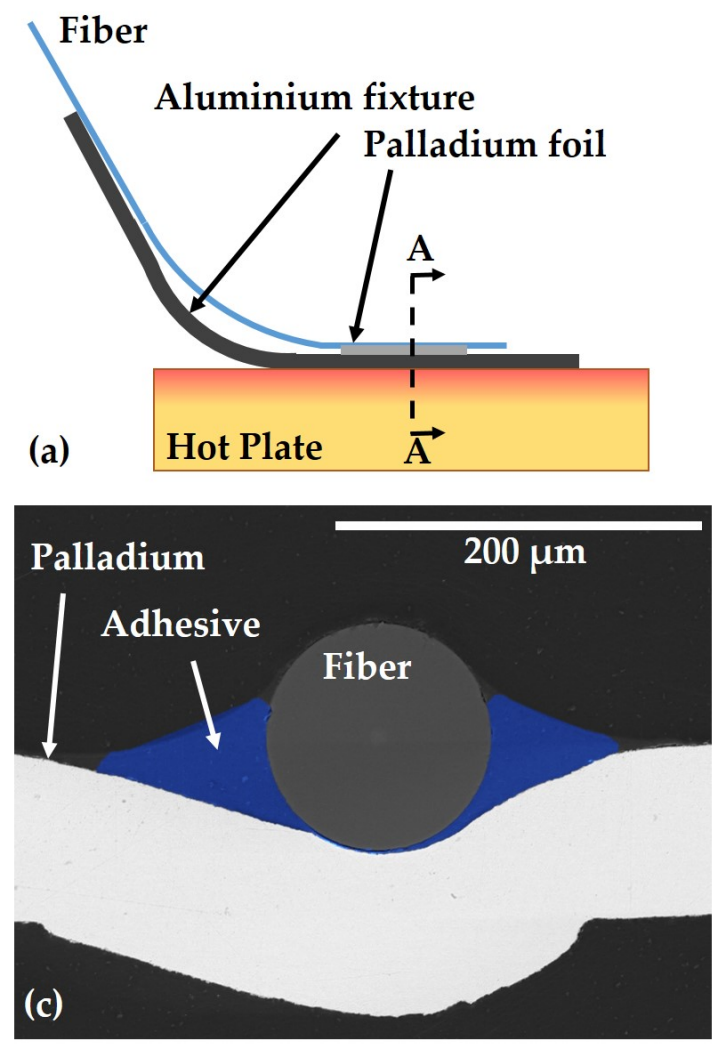

\section{Section A-A}

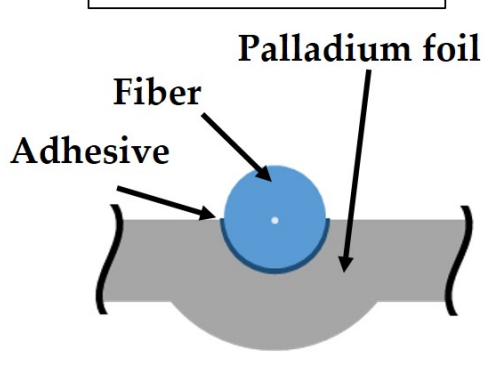

(b)

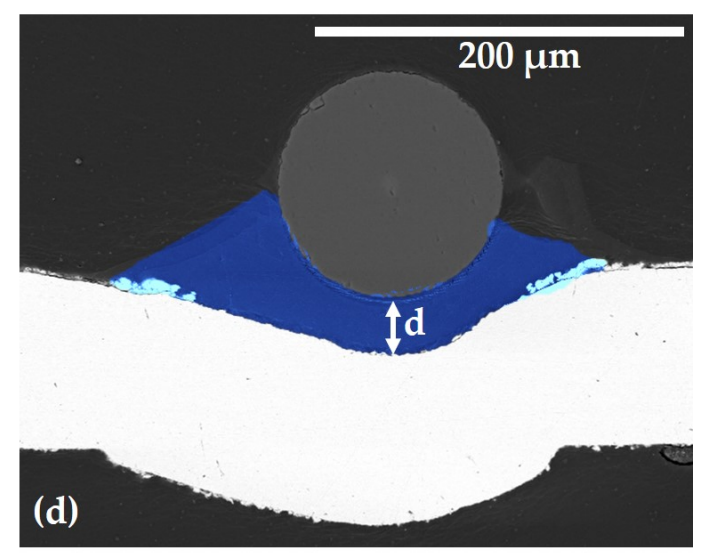

Figure 4.10: Images of the $100 \mu \mathrm{m}$ foil sensor. (a) schematic manufacturing process, (b) schematic cross-sectional view, (c) cross-sectional SEM image with thin bond line, and (d) with thick bond line (adhesive in false colour). 
During vacuum bagging a vacuum is generated between a mould and an air tight film. The air tight film transduces the (atmospheric) pressure difference in a force which is applied on the palladium(foil)-fiber-composite between the mould and the air tight foil. The tool has rectangular grooves enabling the alignment of the foil on the tool (see Figure 4.11 (a)). A V-groove in the center of each rectangular groove enables the alignment of the fiber in the center of the foil. A non-stick PTFE release fabric is applied on the aluminium mould.

The manufacturing process is as follows: Firstly, the formed palladium foil (20 $\mathrm{mm} \times 2 \mathrm{~mm}$ ) is placed in the rectangular groove of the vacuum bagging tool. Secondly, the fiber is aligned in the groove of the foil and a small amount of adhesive is applied (ADR243). Then, the vacuum bagging layers are placed on top. A schematic of this manufacturing process is shown in Figure 4.11 (a). With the vacuum bagging an even force is applied pushing the fiber firmly into the groove and creating a controlled, thin bond line. The vacuum bagging follows common commercial practice and consists of the following layers (see Figure 4.11 (a)). The foil and fiber are covered with a non-stick release film and peel ply. The release film and peel ply are perforated and allow the adhesive to flow to the breather/ bleeder material. The breather/ bleeder material absorbs excessive adhesive and allows full evacuation over the whole area. The vacuum bagging film is the top layer. The vacuum bagging film is sealed to the aluminium mould with vacuum sealant tape. The system can be evacuated causing the atmospheric pressure to apply an even force over the vacuum bagging film on to the fiber. To cure the adhesive the vacuum bagging tool is placed on a hotplate. The surface temperature at the sensor is set to $100^{\circ} \mathrm{C}$ and the adhesive is cured for 3 hours. Vacuum bagging proved to be an excellent method for sensor manufacturing. An SEM analysis demonstrated good fiber groove alignment for the $100 \mu \mathrm{m}$ palladium foil with vacuum bagging (see Figure Figure 4.11 (b)). In addition the bond line thickness is decreased down to below $1 \mu \mathrm{m}$. Furthermore, up to 16 sensor can be manufactured simultaneously with the tool. The repeatability for 15 simultaneously manufactured sensors was in the range of $2 \%$ concerning the sensor response.

With a silicon oxide membrane the cross-sensitivity to carbon monoxide was tried to be improved [69]. After forming the $100 \mu \mathrm{m}$ palladium foils, they were mounted in the holder for sputter coating (see Figure 3.2 (b)). A 100 $\mathrm{nm}$ silicon dioxide layer was sputter coated on both sides of the foil. Afterwards, the sensor manufacturing proceeded with the improved manufacturing method using vacuum bagging. The hydrogen response and cross-sensitivity to carbon monoxide of the sensors with silicon dioxide membrane was tested and compared to sensors without membrane (see chapter 8). 


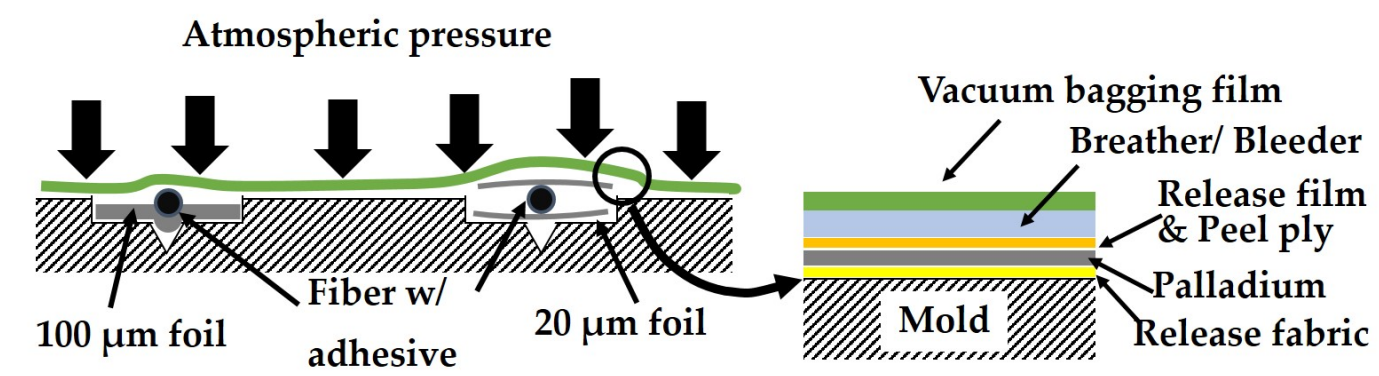

(a)
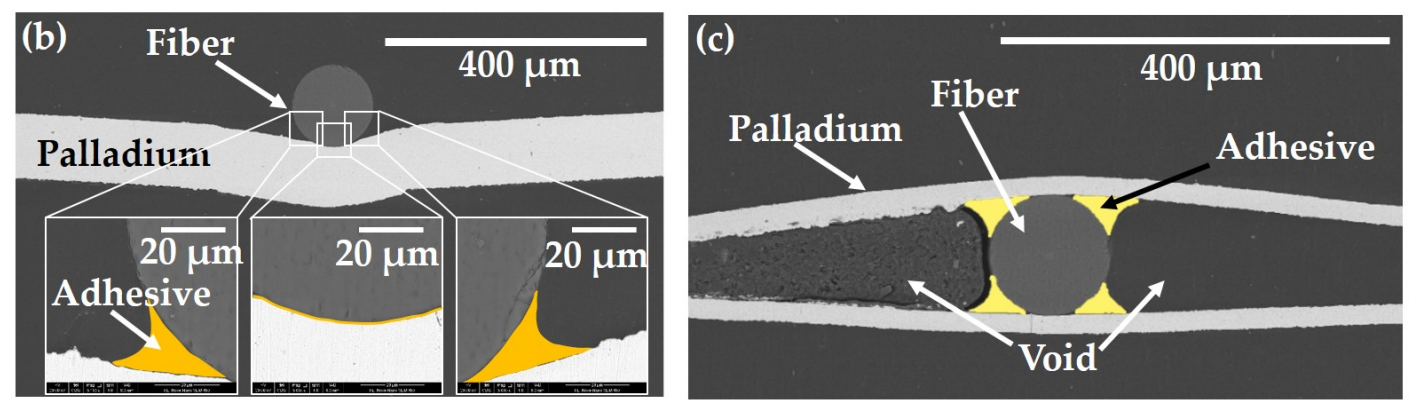

Figure 4.11: Images of the $100 \mu \mathrm{m}$ palladium foil sensor. (a) schematic manufacturing process of the $100 \mu \mathrm{m}$ and sandwiched $20 \mu \mathrm{m}$ palladium foil sensor, (b) SEM cross-sectional view of the $100 \mu \mathrm{m}$ palladium foil sensor (adhesive in false colour), (c) SEM cross-sectional view of the sandwiched $20 \mu \mathrm{m}$ palladium foil sensor (adhesive in false colour).

\subsection{4 $20 \mu \mathrm{m}$ sandwiched palladium foil sensors}

With the development of the vacuum bagging tool, further new sensor designs became technically feasible. A third type of foil sensor that has a fiber sandwiched between two thin, flexible palladium foils is manufactured via the vacuum bagging method. The pressure applied by vacuum bagging bends the foil around the fiber and creates a thin bond line without pre-imprinting the foil. The manufacturing process is as follows: Firstly, one $20 \mu \mathrm{m}$ palladium foil (20 $\mathrm{mm} \times 2 \mathrm{~mm}$, the dimension was chosen to cover the whole length of the FBG and to fit into the slots of the vacuum bagging tool) is placed in the rectangular groove of the tool and the fiber is aligned in the center. Secondly, a small amount of adhesive is applied (ADR243), before the second $20 \mu \mathrm{m}$ palladium foil $(20 \mathrm{~mm} \times 2 \mathrm{~mm}$ ) is placed on top. Then, the vacuum bagging layers are placed on top. The schematic manufacturing process of the sandwiched design is also shown in Figure 4.11 (a). With the vacuum bagging an even force is applied on the palladium foil, forming it around the fiber, and creating a thin bond line (see Figure 4.11 (c)). 


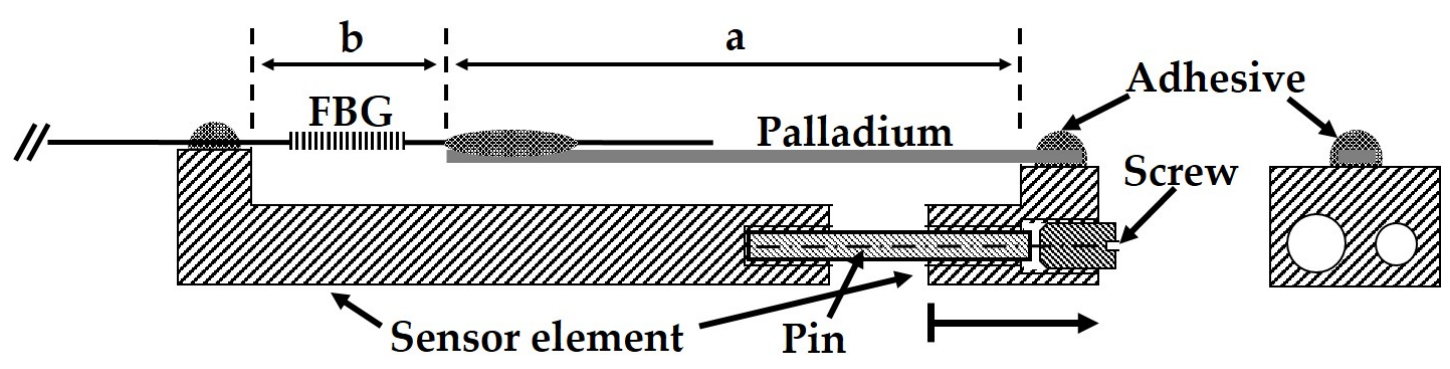

Figure 4.12: Schematic of pre-strained sensor design.

\subsection{5 $10 \mu \mathrm{m}$ sandwiched palladium silver foil sensor}

Apart from the sandwiched $20 \mu \mathrm{m}$ palladium foil sensor a sandwiched sensor with palladium silver foil was developed. This sensor design uses a palladium silver foil with increased hydrogen solubility to improve the sensitivity of the sensor. Palladium silver has an higher hydrogen solubility compared to pure palladium which leads to a increased expansion of the palladium alloy and therefore increased sensitivity of the sensor (see Section 2.2.5 and 4.1.2).

The palladium silver foil is $10 \mu \mathrm{m}$ thick and is made of $75 \%$ palladium and $25 \%$ silver. These flexible palladium silver foils ( $20 \mathrm{~mm} \times 2 \mathrm{~mm}$, the dimension was chosen to cover the whole length of the FBG and to fit into the slots of the vacuum bagging tool) are bonded to the FBG (sandwiched) with the vacuum bagging method as described in Section 4.2.4. With a 25\% silver concentration we would expect an amplification of around 9-17 (see Section 4.1.3).

\subsubsection{Pre-strained $100 \mu \mathrm{m}$ palladium foil sensor}

Another approach to amplify the sensitivity of the sensor is with a new design for mechanical amplification of the expanding palladium. This design concept is novel and original. To our knowledge a sensor based on a pre-strained FBG that measures the expansion of a sensing element with a negative wavelength change has not been reported before. A new approach for strain concentration and to mechanically amplify the expansion of the palladium is to bond a palladium foil to the end of an FBG and bond the assembly to a sensor holder. The FBG is isolated from the palladium and the palladium foil is significantly longer than the isolated length of the FBG. Therefore, a strain concentration of the expanding palladium on the FBG can be achieved. The sensor holder consist of two sensor elements, two pins and an adjustable screw (Figure 4.12). With the screw (M4, 1 whole turn causes $0.7 \mathrm{~mm}$ linear displacement) the distance between the two sensor elements can be altered and the FBG prestrained. 
When the sensor is exposed to hydrogen, the palladium expands due to hydrogen absorption. As a consequence the strain of the FBG is reduced and a negative wavelength change can be detected. In the absence of hydrogen, the palladium desorbs hydrogen, contracts and therefore, again increases the stress on the FBG. The amplification of the sensor design is given by the ratio of palladium foil length $a$ to fiber length $b$. Therefore, the amplification is given by

$$
\varphi=\frac{a}{b}
$$

The length $b$ needs to be at least the length of the FBG and care must be taken that the FBG is not located on the sensor element or foil/bond interface. The length $a$ is chosen to achieve the desired amplification. The amplification, and therefore sensitivity, of the sensor is only limited by the pre-strain of the sensor. When the palladium expansion is equal to the pre-strain, the FBG cannot sense further expansion of the palladium. Therefore, care has to be taken when designing the pre-strained sensor that the expected maximum hydrogen concentration does not lead to a palladium expansion that exceeds the pre-strain. In order to minimize the temperature sensitivity of the sensor the sensor holder elements and pins shown in Figure 4.12 are machined from RoyAlloy (C: 0,05; Si: 0,4:Mn: 1,2; Cr: 12,6; S: 0,12) . RoyAlloy has a similar coefficient of thermal expansion to that of palladium (RoyAlloy: $11 \cdot 10^{-} 6 \mathrm{~m} /(\mathrm{m} \mathrm{K}), \mathrm{Pd}: 11.8 \cdot 10^{-} 6$ $\mathrm{m} /(\mathrm{m} \mathrm{K}))$, creating only a small temperature dependency. The manufacturing of this sensor assembly is as follows: Firstly a $150 \mu \mathrm{m}$ diameter groove (20 $\mathrm{mm}$ length) is imprinted in one end of a $100 \mu \mathrm{m}$ palladium foil ( $2 \mathrm{~mm} \times 100$ $\mathrm{mm}$ ) using the foil forming tool. Secondly, the fiber is aligned in the groove and adhesive (ADR243) is applied on the interface. The adhesive is cured on a hotplate at $100^{\circ} \mathrm{C}$ for 3 hours. Then, the fiber is aligned on the one sensor element, and the foil is aligned on the other sensor element. The fiber and foil are adhesively bonded to the holder as in the previous step. Finally, the fiber is pre-strained by turning the screw and therefore, increasing the gap between the two elements of the sensor holder. The pre-straining is monitored by the peak wavelength shift of the FBG. The pre-strain is set to around $5 \mathrm{~nm}$. After pre-straining, relaxation can be observed by monitoring the peak wavelength. At room temperature the relaxation process took around 3 hours and had a 200 pm wavelength shift. 


\subsection{Sensor calibration}

After manufacturing, each individual sensor needs to be calibrated before it can be used to reliably measure hydrogen concentrations. The straight forward, but also extensive method is a multi-point calibration. A faster method is a single-point calibration, however, for this a precise knowledge of the system is necessary. The hydrogen response of the manufactured sensors can be theoretically predicted by the equations introduced in previous sections. We assume that deviation from the theoretical value (besides uncertainties of constants $k_{\varepsilon}, C$, and the geometrical and material properties $\left.A_{\mathrm{Pd}}, A_{\mathrm{f}}, E_{\mathrm{Pd}}, E_{\mathrm{f}}\right)$ are mainly due to the strain transfer between expanding palladium and fiber. The strain transfer can be addressed and quantified by introducing a coefficient of strain transfer $k_{\mathrm{ST}}$ [140]. The strain of the FBG can be expressed as

$$
\Delta \varepsilon=k_{\mathrm{ST}} \Delta \varepsilon^{\prime}
$$

Inserting this expression into (2.15) leads to

$$
\Delta \lambda=k_{\varepsilon} k_{\mathrm{ST}} \Delta \varepsilon^{\prime}+k_{T} \Delta T
$$

If the strain of the expanding palladium can be fully transduced into the fiber, the coefficient is 1 . If the strain is not fully transduced into the fiber (e.g., due to elasticity of the bonding material, partial delamination of the bonding surface) the coefficient has a value between 0 and 1 . If the palladium does not transduce strain into the fiber (e.g., full delamination of the bonding surface) the coefficient is 0 .

For some sensors investigated in experiments and presented in the following chapters $k_{\mathrm{ST}}$ proved to be larger than 1 , which by definition should not be possible. However, several constants and parameters in the equations presented in Chapter 2 and Chapter 4 have high uncertainties, which can explain this phenomenon (e.g., the scaling factor $C$ has a uncertainty of around $5 \%$ (see Equation (4.1)).

In Chapter 9 three different methods are discussed to determine the coefficient of strain transfer. The most obvious method to determine the coefficient is via a hydrogen measurement. In addition, a new method via temperature measurement is presented that relies on the thermal expansion of palladium. Finally, a third method via strain measurement is described. This conventional method relies on comparing the strain measured with an FBG to the strain measured with a resistive, foil strain gauge. 
Now the hydrogen concentration in gas can be determined with

$$
c_{\mathrm{H} 2 \text { gas }}=\frac{1.013}{p_{\text {total }}} \exp \left(13.04-\frac{2327}{T}-\frac{11110 n}{T}+2 \ln \left(\frac{n}{1-n}\right)\right)
$$

and the dissolved hydrogen concentration in oil with

$$
c_{\mathrm{H} 2 \text { dissolved }}=k_{\text {Ost. }} \frac{1.013}{p_{\text {total }}} \exp \left(13.04-\frac{2327}{T}-\frac{11110 n}{T}+2 \ln \left(\frac{n}{1-n}\right)\right)
$$

where $p_{\text {total }}$ is the ambient pressure in bar, $T$ is the temperature in Kelvin, $k_{\text {Ost. }}$ is the Ostwald coefficient and $n$ is

$$
n=\frac{\Delta \lambda}{C k_{\varepsilon} k_{\mathrm{ST}}} \frac{1+Y}{Y} .
$$




\section{Chapter 5}

\section{Coated and first generation foil sensors}

In this chapter the results of the coated sensors and the first generation wrapped $20 \mu \mathrm{m}$ and $100 \mu \mathrm{m}$ foil sensors using the initial manufacturing methods (see Sections 4.2.2 and 4.2.3) are presented and discussed. In this chapter we show the sensitivity limitations of the coated sensors, and the sensitivity increase using the palladium foil sensors. Furthermore, we highlight the deviation of the measured from the calculated sensitivity, which implies that potential exists for further increase in sensitivity.

\subsection{The coated sensor}

Two coated sensors (C1 and C2) were manufactured according to Section 4.2.1 and tested over a period of 3 months in changing hydrogen-nitrogen-mixtures and at different temperatures. On day 1 testing was performed at $30.1 \pm 0.1^{\circ} \mathrm{C}$ with a gas mixture of 2500 ppm hydrogen. On day 2 testing was performed at $90.2 \pm 0.23^{\circ} \mathrm{C}$ for a concentration range of $1-5 \%$ hydrogen. Afterwards the sensors were kept in pure nitrogen atmosphere at $90^{\circ} \mathrm{C}$. On day 32 testing was performed at $89.6 \pm 0.23^{\circ} \mathrm{C}$ and $5 \%$ hydrogen. Afterwards, the sensors were again kept in nitrogen atmosphere at $90^{\circ} \mathrm{C}$. On day 82 testing was performed at $90.2 \pm 0.3^{\circ} \mathrm{C}$ for $1-5 \%$ hydrogen. This was followed by testing at $60.1 \pm 0.3^{\circ} \mathrm{C}$ and $120.4 \pm 0.3^{\circ} \mathrm{C}$ for $1-5 \%$ hydrogen. This testing sequence was chosen, because on day two the response of the two sensors differed significantly. We assumed that a relaxation, or annealing process at $90^{\circ} \mathrm{C}$ over several days would have an positive impact. On day 32 the hydrogen response was found to be similar for the two sensors. No further improvement was observed at day 82 . Therefore, the characterisation of the sensor was performed following day 82. During the 


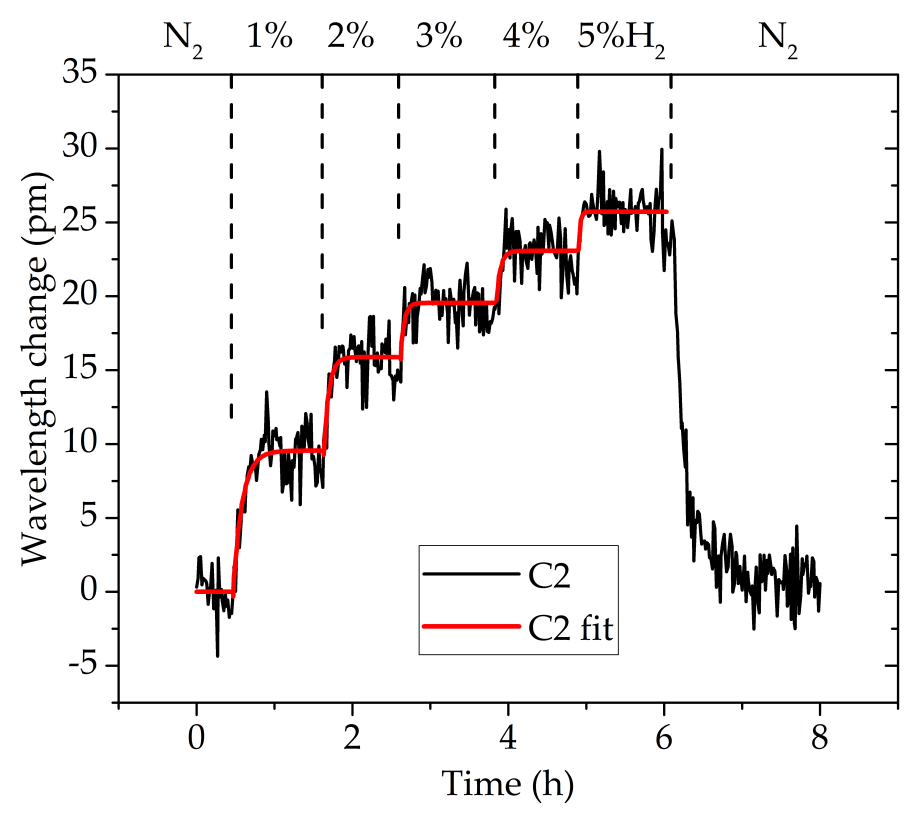

Figure 5.1: Sensor signal of the coated sensor C2 on day 82 at $90^{\circ} \mathrm{C}$ for a concentration range of $1-5 \%$ hydrogen and the corresponding fit.

hydrogen testing the pressure in the chamber was $1065 \pm 5$ mbar. To determine the final wavelength change of each hydrogen measurement and the response time a function following an exponential decay $\left(y=a^{*} \exp \left(b^{*} x\right)+c\right)$ was fitted to the data. This fitting function was selected since the equations (2.9) given in Section 2.2.3 can be well approximated by an exponential decay function [112].

\subsubsection{Results}

Figure 5.1 shows a sensor signal of the coated sensor $\mathrm{C} 2$ on day 82 at $90^{\circ} \mathrm{C}$ for a concentration range of $1-5 \%$ hydrogen and the corresponding fit (Sensor C1 behaves similarly).

Figure 5.2 shows the initial (day 2) and final (day 82) response of both sensors $\mathrm{C} 1$ and $\mathrm{C} 2$ to $1-5 \%$ hydrogen at $90^{\circ} \mathrm{C}$. Initially, $\mathrm{C} 2$ had a significantly lower response than $\mathrm{C} 1$. After 82 days the response of both sensors is similar. The $5 \%$ hydrogen testing on day 2, 32 and 82 indicates that equalisation occurred before day 32 , since there is no further increase in response after day 32.

Figure 5.3 shows the hydrogen response of sensor $C 2$ to $1-5 \%$ hydrogen at different temperatures. Also displayed is the calculated hydrogen response of the sensor C2 (dashed line), as described in Section 9.1 with a coefficient of strain transfer $k_{\mathrm{ST}}$ of 0.34 . Here, the value for $k_{\mathrm{ST}}$ of the sensor C2 was obtained by averaging $k_{\mathrm{ST}}$ calculated for each hydrogen concentration and temperature. At $30^{\circ} \mathrm{C}$ only $2500 \mathrm{ppm}$ hydrogen were tested to avoid the phase change re- 


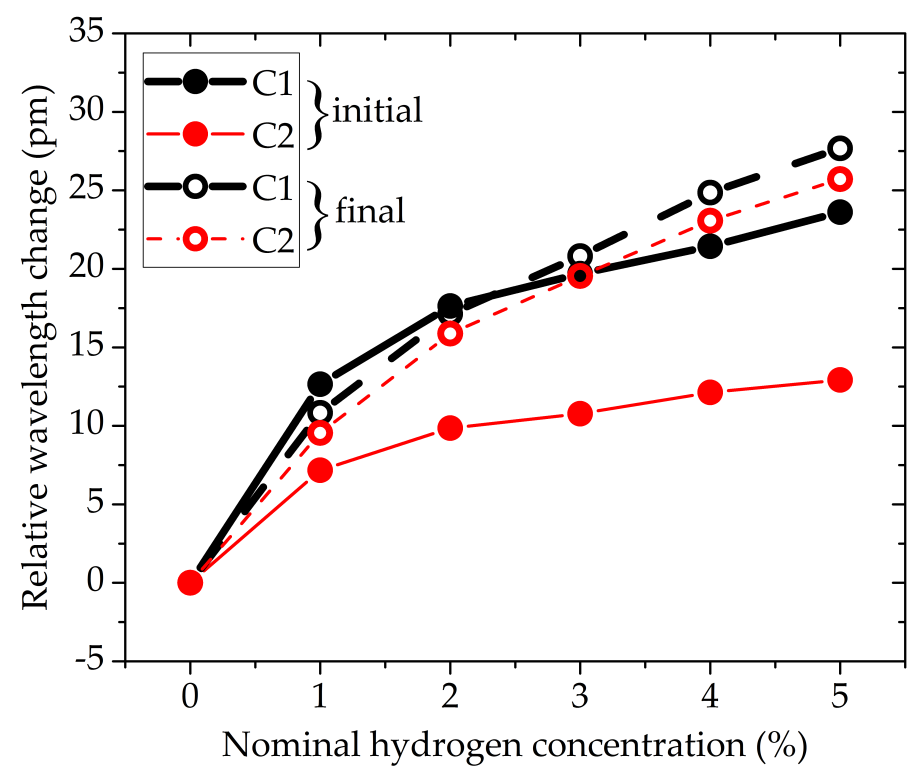

Figure 5.2: Response to $1-5 \%$ hydrogen at $90^{\circ} \mathrm{C}$ of the coated sensor $\mathrm{C} 1$ (black) and C2 (red) after 2 (solid) and 82 (dashed) days.

gion. The $99 \%$ response time (99\% of the final response is reached, derived from the fitting function), of both sensors is similar and decreases with increasing temperature. It is around $40 \mathrm{~min}$ for a 0 to $5 \%$ hydrogen step at $90^{\circ} \mathrm{C}$.

Figure 5.4 shows the temperature sensitivity of a bare reference fiber (10.95 $\left.\mathrm{pm} /{ }^{\circ} \mathrm{C}\right)$, the coated sensors $\mathrm{C} 1\left(11.61 \mathrm{pm} /{ }^{\circ} \mathrm{C}\right)$, and $\mathrm{C} 2\left(11.52 \mathrm{pm} /{ }^{\circ} \mathrm{C}\right)$, and the calculated maximum sensitivity of the coated sensors $\left(12.38 \mathrm{pm} /{ }^{\circ} \mathrm{C}\right)$. The value $k_{\mathrm{ST}}=1$ was used to calculate the maximum temperature sensitivity (see Section 9.2). The deviation between measured and calculated sensitivity is due to $k_{\mathrm{ST}} \neq 1$ for $\mathrm{C} 1$ and $\mathrm{C} 2$.

\subsubsection{Discussion}

The coated sensor C1 was stable over 82 days. The coated sensor C2 improved its sensitivity over time. Eventually both coated sensors showed a similar response to hydrogen (compare Figure 5.2). Possible reasons for the improvement in sensitivity of sensor $\mathrm{C} 2$ are that (a) the adhesion between the fiber and the titanium layer, and/ or between the titanium layer and the palladium layer improved and therefore, more strain was transduced into the fiber, or alternatively, (b) annealing the palladium itself improved the hydrogen absorption and therefore increases the expansion and response. However, even after this improvement the $k_{\mathrm{ST}}$ for $\mathrm{C} 1$ and $\mathrm{C} 2$ are around 0.38 and 0.34 , which implies that only around a third of the expansion of the palladium is transfered to the 


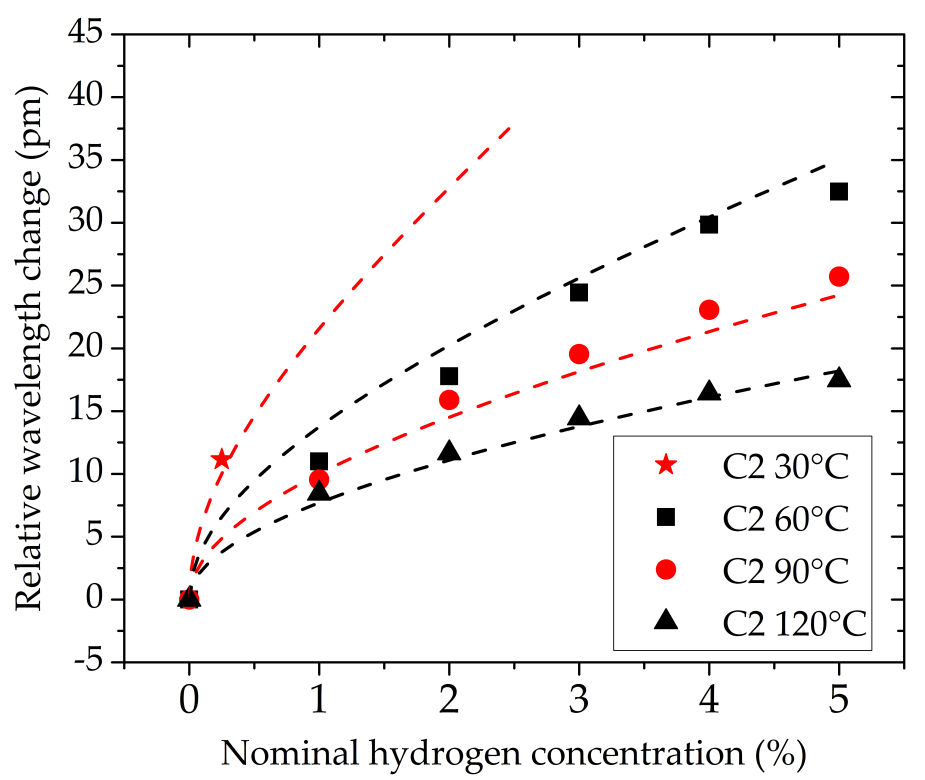

Figure 5.3: Calculated response (dashed line) with $k_{\mathrm{ST}}=0.34$ and experimental data (scatter) of the coated sensor C2 at various hydrogen concentrations and temperatures.

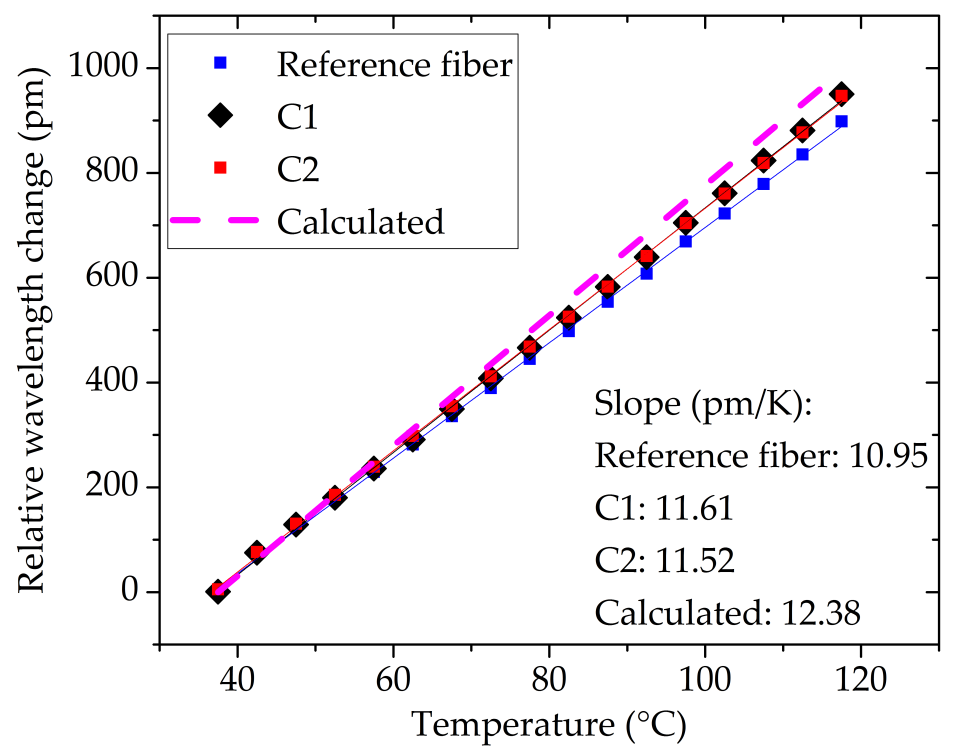

Figure 5.4: Temperature sensitivity of the coated sensors $C 1$ and $C 2$ and the uncoated reference fiber. Also shown the maximum, calculated temperature sensitivity of the coated fiber. 
FBG. This is unexpected, as we use a $30 \mathrm{~nm}$ titanium layer to improve the adhesion and strain transfer between palladium and fiber. Unfortunately, comparing the adhesion to other in literature reported sensors is not possible, since the coefficient of strain transfer has not been determined for other hydrogen sensors. The adhesion might be improved, by a surface treatment of the fiber (e.g., micro-structuring $[75,158]$ or by changing the adhesion layer parameters (e.g., thickness, and material $[43,116])$.

The exponential fit describes the hydrogen response of the coated sensors well (see 5.1). Examining Figure 5.1 also shows a moderate signal to noise ratio of around 4, which is influenced by the wavelength accuracy of the optical equipment, the data processing approach and the relatively low signal of the coated sensor.

The coated sensors showed the expected temperature dependency of the hydrogen sensitivity. Lower temperatures result in a higher hydrogen sensitivity. The wavelength change of sensor $\mathrm{C} 2$ due to $1 \%$ hydrogen is equivalent to the wavelength change due to $1^{\circ} \mathrm{C}$ at $60^{\circ} \mathrm{C}, 90^{\circ} \mathrm{C}$ and $120^{\circ} \mathrm{C}$. The wavelength change due to $5 \%$ is equivalent to wavelength change due to $3^{\circ} \mathrm{C}$ at $60^{\circ} \mathrm{C}, 2.5^{\circ} \mathrm{C}$ at $90^{\circ} \mathrm{C}$ and $1.5^{\circ} \mathrm{C}$ at $120^{\circ} \mathrm{C}$. This implies a high temperature cross-sensitivity of the coated sensors.

\subsection{The wrapped $20 \mu \mathrm{m}$ and $100 \mu \mathrm{m}$ foil sensors with the initial manufacturing methods}

The foil sensors were manufactured according to Sections 4.2.2 and 4.2.3. For these sensors, two different adhesives were tested: The NOA83H and the $\mathrm{NOA} 86 \mathrm{H}$ (see Section 3.1.5). One objective of this experiment was a proof of concept of the two sensor designs. The other objective was to determine an appropriate adhesive to bond the foils to the fiber. Both adhesives used are advertised to withstand temperatures $>125^{\circ} \mathrm{C}$. However, as we later found out, their glass transition temperature is $<125^{\circ} \mathrm{C}$. The manufactured sensors with corresponding adhesives are listed in Table 5.1.

The foil sensors were tested over a period of 2 months $\left(90.5 \pm 0.5^{\circ} \mathrm{C}, \mathrm{c} 1065 \pm 5\right.$ mbar), firstly by cycling hydrogen and nitrogen (after each hydrogen concentration the sensors were unloaded in nitrogen) with changing hydrogen concentration $(5,1,2,3,4,5 \%$ hydrogen), and then by increasing the hydrogen concentration from 0 to $5 \%$ in $1 \%$ steps. Finally, after 30 days under hydrogen the sensors were unloaded in nitrogen and then again tested in two 5\% hydrogennitrogen-cycles. The test sequence was chosen, to (a) characterise the sensors 
Table 5.1: List of sensors for experiment: $20 \mu \mathrm{m}$ (wrapped) and $100 \mu \mathrm{m}$ palladium foil sensors with initial manufacturing methods

\begin{tabular}{lll}
\hline Name & Foil & Adhesive \\
\hline F1 & $20 \mu \mathrm{m}$ Pd foil & NOA83H \\
F2 & $20 \mu \mathrm{m}$ Pd foil & NOA83H \\
T1 & $100 \mu \mathrm{m}$ Pd foil & NOA83H \\
T2 & $100 \mu \mathrm{m}$ Pd foil & NOA83H \\
T3 & $100 \mu \mathrm{m}$ Pd foil & NOA86H \\
T4 & $100 \mu \mathrm{m}$ Pd foil & NOA86H \\
\hline
\end{tabular}

over a hydrogen range from 0 to $5 \%$ and to $(\mathrm{b})$ determine the unloading behaviour of the sensors after long term hydrogen exposure. Since a change in sensitivity was observed after the unloading of the sensors, two additional $5 \%$ hydrogen-nitrogen-cycles were conducted.

\subsubsection{Results}

Figure 5.5 shows the signal of sensor T4 on days $8-11$ at $90^{\circ} \mathrm{C}$ for the step increases from 0 to $5 \%$ hydrogen. Also displayed is the corresponding exponential fit. The other sensors show similar behaviour.

Figure 5.6 shows the response to $5 \%$ at $90^{\circ} \mathrm{C}$ of the different foil sensors at different days during the test period. It appeared that sensors F1, T1, and T2 were not stable over the whole experiment. In the further discussion we focus on the sensors F2, T3, and T4 which were stable during the examination.

Figure 5.7 shows the hydrogen response of sensor F2, T3 and $\mathrm{T} 4$ at $90^{\circ} \mathrm{C}$. The experimental values from hydrogen cycling (unloading with nitrogen after each loading cycle) and hydrogen step increase are displayed as unfilled and filled symbols respectively. The experimental data only shows minor deviation from the calculated hydrogen response (dashed lines). $k_{\mathrm{ST}}$ for each sensor was obtained by averaging the calculated value of $k_{\mathrm{ST}}$ of each hydrogen step. The response time, when $99 \%$ of the final hydrogen response is reached (derived from the exponential fit), of the foil sensors is around 4 hours for the $20 \mu \mathrm{m}$ and around 8 hours for the $100 \mu \mathrm{m} \mathrm{Pd}$ foil sensor for a 0 to $5 \%$ hydrogen step at $90^{\circ} \mathrm{C}$.

Figure 5.8 shows the temperature sensitivity of the reference fiber (10.86 $\left.\mathrm{pm} /{ }^{\circ} \mathrm{C}\right)$, the $20 \mu \mathrm{m}$ foil sensor F2 $\left(20.87 \mathrm{pm} /{ }^{\circ} \mathrm{C}\right)$, the $100 \mu \mathrm{m}$ foil sensors T3 $\left(16.12 \mathrm{pm} /{ }^{\circ} \mathrm{C}\right)$ and $\mathrm{T} 4\left(14.71 \mathrm{pm} /{ }^{\circ} \mathrm{C}\right)$, and the calculated maximum temper- 


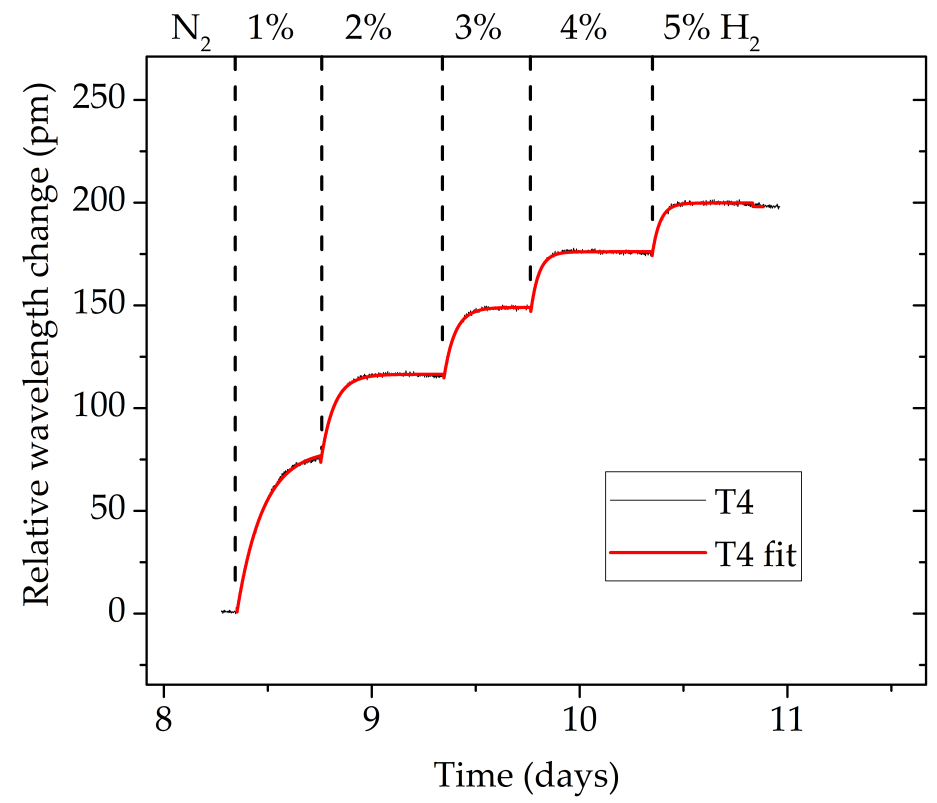

Figure 5.5: Sensor signal of foil sensor $\mathrm{T} 4$ and fit at $90{ }^{\circ} \mathrm{C}$. The black line is the measures signal (wavelength stability $\pm 2 \mathrm{pm}$ ), the red line is the fit (exponential decay).

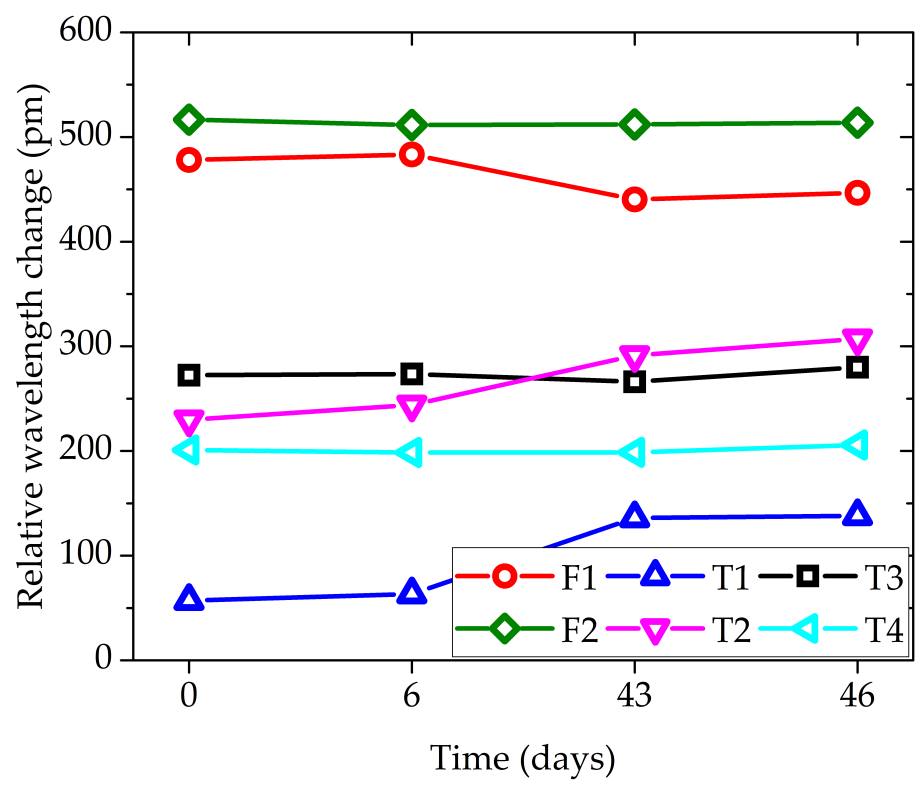

Figure 5.6: Response of the foil sensors to $5 \%$ hydrogen at $90^{\circ} \mathrm{C}$ on days 2,6 , 43 and 48. 


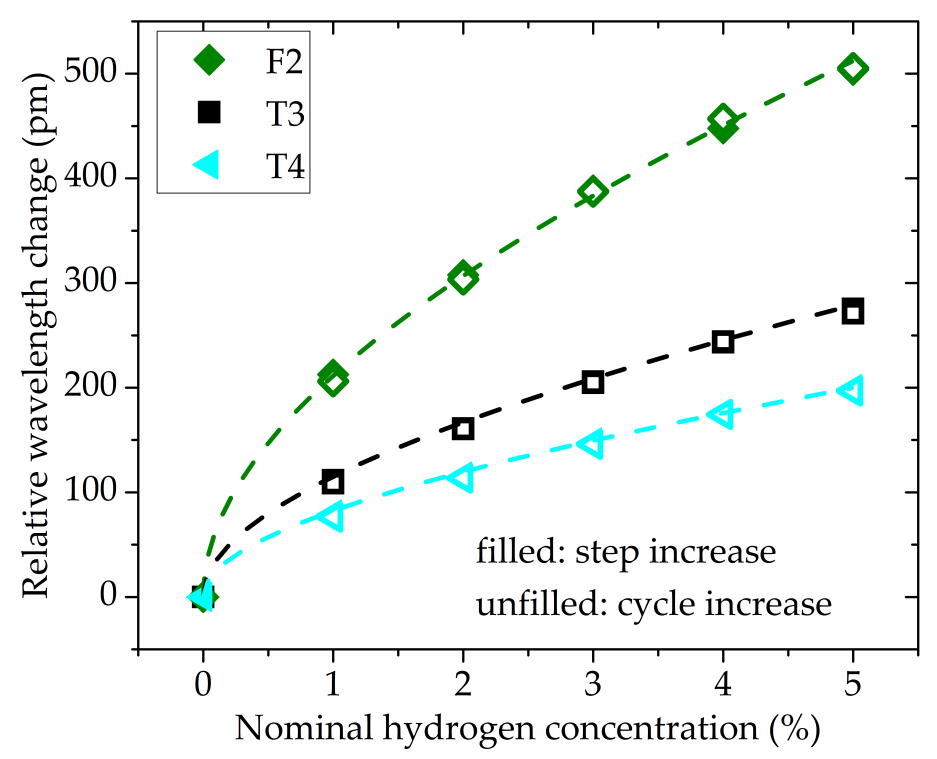

Figure 5.7: The calculated response (dashed line) and experimental determined response (points) of the foil sensors F2, T3 and T4 to 1-5\% hydrogen at $90^{\circ} \mathrm{C}$.

ature sensitivity with $k_{\mathrm{ST}}=1\left(21.27 \mathrm{pm} /{ }^{\circ} \mathrm{C}\right.$ for the $20 \mu \mathrm{m}$ foil sensor and 24.62 $\mathrm{pm} /{ }^{\circ} \mathrm{C}$ for the $100 \mu \mathrm{m}$ foil sensor). The deviation between measured and calculated maximum sensitivity is again due to $k_{\mathrm{ST}} \neq 1$ for the sensors F2, T3, and T4.

\subsubsection{Discussion}

The foil sensors F2, T3 and T4 were stable over the period of the experiment. The sensors F1, T1 and T2 were not stable over time (Figure 5.6). A possible reason is a post curing of the adhesive. This implies that the initial curing was not long enough and/or the curing temperature was to low. The foil sensors of each type were manufactured in the same way as described in Chapter 4 . However, since the processes are not automated, small differences in curing time, temperature and positioning of the sensor on the hotplate are likely and can explain the different post curing behaviour. For sensors T1 and T2 the adhesive NOA83H was used and for the sensors T3 and T4 the adhesive NOA86H. The sensors T1 and T2 showed post curing behaviour whereas the sensors T3 and T4 did not. This implies a correlation between choice of adhesive and post curing, however post curing was not observed on sensor F2 which also uses $\mathrm{NOA} 83 \mathrm{H}$ as an adhesive.

For the foil sensors, the exponential fit describes the hydrogen response 


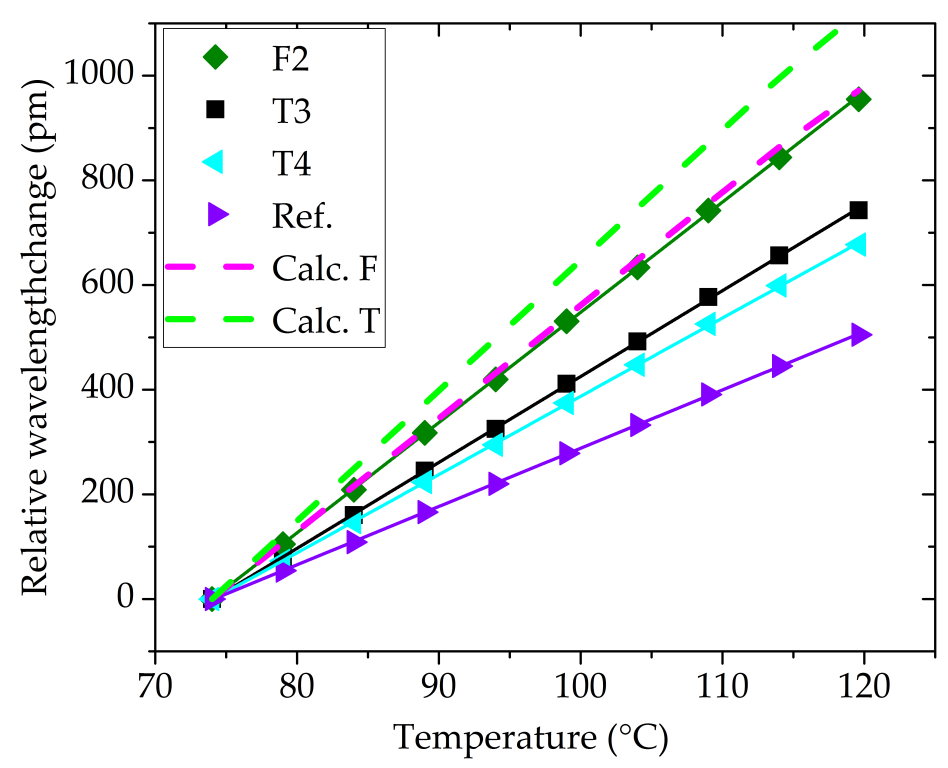

Figure 5.8: Temperature response of the foil sensors F2, T3 and T4 and the uncoated reference fiber. Also shown are the calculated temperature response of the foil sensors $\mathrm{F}$ and $\mathrm{T}$.

well (see Figure 5.5). The signal to noise ratio is $>50$ for all foil sensors.

The wavelength change of sensor F2, T3, and T4 due to $1 \%$ hydrogen at $90^{\circ} \mathrm{C}$ is equivalent to the wavelength change due to $10^{\circ} \mathrm{C}, 7^{\circ} \mathrm{C}$, and $5^{\circ} \mathrm{C}$ respectively. The wavelength change of sensor F2, T3, and T4 due to $5 \%$ hydrogen at $90^{\circ} \mathrm{C}$ is equivalent to the wavelength change due to $24^{\circ} \mathrm{C}, 17^{\circ} \mathrm{C}$, and $13^{\circ} \mathrm{C}$ respectively. The hydrogen-nitrogen cycles and hydrogen step increase show similar results (Figure 5.7). This suggests high consistency of the individual foil sensors. The $k_{\mathrm{ST}}$ for the foil sensors are $0.97,0.39$, and 0.28 for the sensors F2, T3, and T4 respectively. This implies that especially for the $100 \mu \mathrm{m}$ foil sensors only around a third or less of the expansion of the palladium is straining the FBG.

\subsection{Comparison of the performance of the coated versus the foil sensors}

In this section we compare the results of the coated and the foil sensors. The sensitivity to hydrogen and temperature of all the sensors is listed in Table 5.2. The hydrogen sensitivity was obtained for a $1 \%$ hydrogen step at $90^{\circ} \mathrm{C}$. The coated sensors have a sensitivity of around $10 \mathrm{pm} / \%$, which is up to 21 times 
Table 5.2: Hydrogen and temperature sensitivity, and resolution and accuracy of the coated and foil sensors. The hydrogen sensitivity was obtained for a $1 \%$ hydrogen step at $90{ }^{\circ} \mathrm{C}$. The resolution and accuracy was obtained for $1 \%$ hydrogen step at $90^{\circ} \mathrm{C}$

\begin{tabular}{lrrrrr}
\hline Sensitivity & $\mathrm{C} 1$ & $\mathrm{C} 2$ & $\mathrm{~F} 2$ & $\mathrm{~T} 3$ & $\mathrm{~T} 4$ \\
\hline Hydrogen $\left(\mathrm{pm} / \% \mathrm{H}_{2}\right)$ & 10.9 & 9.6 & 212.6 & 111.5 & 78.7 \\
Temperature $\left(\mathrm{pm} /{ }^{\circ} \mathrm{C}\right)$ & 11.6 & 11.5 & 20.9 & 16.1 & 14.7 \\
\hline Resolution $\left(\% \mathrm{H}_{2}\right)$ & 0.17 & 0.19 & 0.009 & 0.016 & 0.023 \\
Accuracy $\left( \pm \% \mathrm{H}_{2}\right)$ & 0.51 & 0.57 & 0.03 & 0.05 & 0.07 \\
\hline
\end{tabular}

lower than the foil sensors. Of the foil sensors the $20 \mu \mathrm{m}$ Pd foil sensor has the highest sensitivity with $212.6 \mathrm{pm} / \% \mathrm{H}_{2}$ followed by the $100 \mu \mathrm{m}$ foil sensors with 78.8 and $111.5 \mathrm{pm} / \%$. The temperature sensitivity of the sensors varies from $11.5-20.9 \mathrm{pm} /{ }^{\circ} \mathrm{C}$.

The resolution of the sensor is calculated by dividing the wavelength resolution of the optical spectrum analyzer (around $1 \mathrm{pm}$ ) by the slope of the sensor response of Figure 5.3 and Figure 5.7. At $1 \%$ hydrogen and $90^{\circ} \mathrm{C}$ the resolution is around $0.17 \%$ hydrogen for C1, $0.19 \%$ hydrogen for C2, $0.009 \%$ hydrogen for F2, $0.016 \%$ hydrogen for T3 and $0.023 \%$ hydrogen for T4. However, the sensitivity, and therefore resolution decreases with increasing hydrogen concentration. This is due to the fact that the mole ratio $n=\mathrm{H} / \mathrm{Pd}$, and therefore, the hydrogen response does not increase linearly with increasing hydrogen concentration (see Chapter 4). The accuracy of the sensor can be calculated by dividing the accuracy of the optical measurement and data processing ( $\pm 3 \mathrm{pm})$ by the slope of the sensor response. At $1 \%$ hydrogen and $90^{\circ} \mathrm{C}$ the resolution is around $\pm 0.51 \%$ hydrogen for $\mathrm{C} 1, \pm 0.57 \%$ hydrogen for $\mathrm{C} 2, \pm 0.03 \%$ hydrogen for $\mathrm{F} 2, \pm 0.05 \%$ hydrogen for $\mathrm{T} 3$ and $\pm 0.07 \%$ hydrogen for $\mathrm{T} 4$.

Overall, the hydrogen sensitivity of the foil sensors is significantly higher than that of the coated sensors. This can be explained with the increased CSA ratio of the foil sensors compared to the coated sensors (compare Figure 4.4). Generally, the following rule applies: the thicker the Pd layer the more sensitive is the sensor. However, the thicker the Pd layer is chosen the slower the response time becomes due to the longer diffusion time within the Pd. The sensitivity of the $20 \mu \mathrm{m} \mathrm{Pd}$ foil sensor is higher than the sensitivity of the 100 $\mu \mathrm{m}$ palladium foil sensors. This is in contradiction of the calculations, but this can be explained by the imperfect strain transfer. $k_{\mathrm{ST}}$ is larger for the $20 \mu \mathrm{m}$ pal- 
ladium foil sensors which implies the strain transfer of the $20 \mu \mathrm{m}$ palladium foil into the fiber is considerably higher than that of the $100 \mu \mathrm{m}$ palladium foil. The fact that the $20 \mu \mathrm{m}$ palladium foil sensor has a higher $k_{\mathrm{ST}}$ might be due to higher pressure applied during its manufacturing process creating a thinner bond line compared to the $100 \mu \mathrm{m}$ palladium foil sensors (compare Figure 4.10 and Figure 4.7)

Comparing the presented sensors to other reported hydrogen sensor, the foil sensors have similar measuring range, and accuracy [65]. Literature values for (pure) palladium coated FBG are mainly obtained at room temperature and range from $20-80 \mathrm{pm} / \% \mathrm{H}_{2}[124,127]$. Predicting the response to $1 \%$ hydrogen according to Figure 5.3 the sensitivity of the presented coated sensor is around $20 \mathrm{pm} / \% \mathrm{H}_{2}$ at $30{ }^{\circ} \mathrm{C}$. Literature values for sensors based on FBG with adhesively bonded palladium bodies are $300 \mathrm{pm} / \% \mathrm{H}_{2}$ at $95^{\circ} \mathrm{C}$ for a palladium tube (wall thickness $33 \mu \mathrm{m}$ ) [131] and $290 \mathrm{pm} / \% \mathrm{H}_{2}$ at $80^{\circ} \mathrm{C}$ for a side polished FBG with a $2 \mu \mathrm{m}$ palladium foil [79].

\subsection{Conclusion}

All sensors showed a hydrogen response. Compared to the foil sensors, the sensitivity of the coated sensors is low and the cross sensitivity to temperature is high. Furthermore, even in the lab environment the inaccuracy is larger than $\pm 0.5 \%$ hydrogen which makes them unsuitable for the practical application for sensing hydrogen in a transformer. An accuracy of $\pm 0.5 \%$ hydrogen in gas is equivalent to an accuracy of \pm 250 ppm dissolved hydrogen in oil (Ostwald coefficient 0.05 ), which makes a classification as described in Section 2.1.4 challenging. Even with possible improvement in the strain transfer between palladium and fiber, the accuracy would be too low for this application.

The foil sensors seem to be a promising solution for hydrogen sensing at low concentration. The sensitivity is significantly higher compared to the coated sensors and the cross-sensitivity to temperature is lower. The accuracy of all presented foil sensors would be high enough for the practical application for sensing hydrogen in a transformer. There is also potential for improvement of the the foil sensors by: (a) Choosing an adhesive with better curing behaviour to eliminate the chance of accidental post-curing. In addition, the adhesive should be chosen so that the glass transition temperature is above the maximum expected working temperature (both adhesive used in this chapter have a glass transition $<45^{\circ} \mathrm{C}$ ). (b) Improving the manufacturing method, to improve the curing of the adhesive and control the bond line thickness (compare Figure $4.10(\mathrm{c}),(\mathrm{d}))$. 
In conclusion, the foil sensors seemed to be a promising solution for the hydrogen monitoring in transformers, and were thus further developed. 


\section{Chapter 6}

\section{Second generation foil sensors}

In this chapter the results of three sensors are presented, discussed and compared to the first generation foil sensors. The improved wrapped $20 \mu \mathrm{m}$ palladium foil sensor with the new manufacturing method using a foil wrapping tool (see Section 4.2.2). The improved $100 \mu \mathrm{m}$ palladium foil sensor with the new manufacturing method using vacuum bagging (see Section 4.2.3). The new sandwiched $20 \mu \mathrm{m}$ palladium foil sensor with the new manufacturing method also using vacuum bagging (see 4.2.4).

All sensors were tested simultaneously in the same test chamber over a period of around 68 days. During that period the test conditions were stable at $90.2 \pm 0.7^{\circ} \mathrm{C}$ and $1062 \pm 10$ mbar. Between hour 330 and 380 the circulation pump stopped causing a slight temperature $\left(91.5 \pm 0.5^{\circ} \mathrm{C}\right)$ and pressure (1090 \pm 5 mbar) increase. The test sequence was as follows; starting in nitrogen, hydrogen concentration steps to $0.25 \%, 1 \%$ and $5 \%$ hydrogen in nitrogen were performed, and finally, the sensors were unloaded in air. Following this test the sensors were cycled three time in 5\% hydrogen in nitrogen and air to determine the repeatability and response time. The test sequence was chosen to characterise the sensors in a range of $0.25 \%$ to $5 \%$ hydrogen. Each step was held for several days, since the sensors were tested simultaneously with the sensors presented in Chapter 7, of which the palladium silver sensor had a very slow response. The following three $5 \%$ hydrogen cycles were performed to test the repeatability of the measurement.

To improve the peak detection a depolarizer was used during this set of experiments. The depolarizer serves the purpose of depolarizing the incident light of the polarized light source which was thought to reduce the effect of birefringence that is common for surface bonded FBGs. However, adding the depolarizer caused an increase in noise due to its temperature sensitivity and daily temperature fluctuations. This effect can be seen in Figure 6.1 and becomes obvious when comparing it to Figure 5.5. In conclusion, we refrained 
from using the depolarizer after this experiment, since the influence of birefringence on the peak detection is minor compared to the effect of the temperature sensitivity of the depolarizer.

\subsection{The wrapped $20 \mu \mathrm{m}$ palladium foil sensor with improved manufacturing process}

Three wrapped $20 \mu \mathrm{m}$ palladium foil sensors were manufactured according to Section 4.2.2 using a wrapping tool and ADR 243 as an adhesive. ADR 243 has a heat deflection temperature of $135^{\circ} \mathrm{C}$ which indicates a better thermal stability compared to the adhesive used for the first generation foil sensors. One sensor broke during installation in the test chamber, but the remaining two sensors behaved similarly.

\subsubsection{Results}

The peak wavelength shift of one wrapped $20 \mu \mathrm{m}$ Pd foil sensor due to $0.25 \%$, $1 \%$ and $5 \%$ hydrogen in nitrogen is shown in Figure 6.1. The final response to the hydrogen steps is 126, 252, and 606 pm respectively. After reaching the maximum at the $5 \%$ step, the peak wavelength decreased by $53 \mathrm{pm}$ to 553 pm. After unloading, the offset from the starting wavelength was $-39 \mathrm{pm}$. In the following three $5 \%$ hydrogen cycles the wavelength change was repeatable with $589 \pm 3$ ppm and the response time was around 30 minutes. The calculated peak wavelength shifts of the sensor due to $0.25 \%, 1 \%$ and $5 \%$ hydrogen are 118,242 , and 587 pm respectively. The deviation of the calculated response from the actual response is due to $k_{\mathrm{ST}} \neq 1$.

The sensitivity of both sensors is displayed in Figure 6.2. Also displayed is the calculated sensitivity with $k_{\mathrm{ST}}=0.99$. In the final three $5 \%$ hydrogen cycles the response of the two sensors was repeatable with $575 \pm 2 \mathrm{ppm}$ and $589 \pm 3$ ppm. This leads to $k_{\mathrm{ST}}=0.98$ and $k_{\mathrm{ST}}=1.00$ The temperature sensitivity of the sensors was $20.8 \mathrm{pm} /{ }^{\circ} \mathrm{C}$ and $21.6 \mathrm{pm} /{ }^{\circ} \mathrm{C}$.

\subsubsection{Discussion}

The wavelength change of the sensor due to $1 \%$ hydrogen at $90^{\circ} \mathrm{C}$ is equivalent to the wavelength change caused by a temperature change of over $12^{\circ} \mathrm{C}$. This implies low temperature cross sensitivity and is an improvement of $17 \%$ compared to the sensor with the initial manufacturing process. 


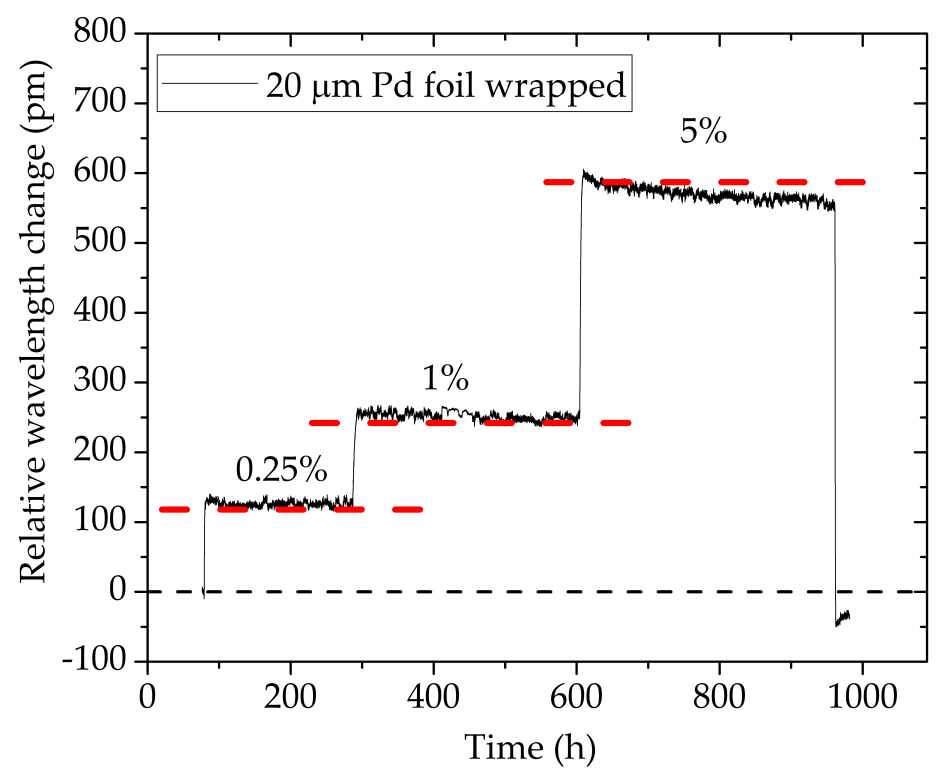

Figure 6.1: Sensor signal of a wrapped $20 \mu \mathrm{m}$ Pd foil sensor at $90^{\circ} \mathrm{C}$ for three different hydrogen concentrations. Also displayed is the calculated response for each concentration.

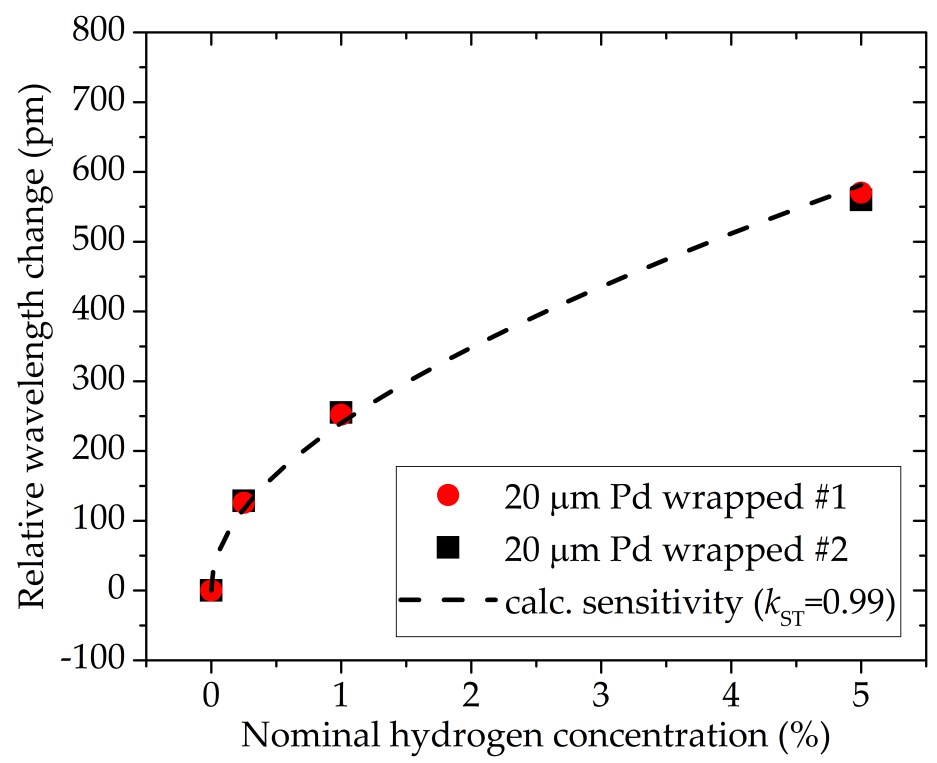

Figure 6.2: Sensitivity of the wrapped $20 \mu \mathrm{m} \mathrm{Pd}$ foil sensors at $90^{\circ} \mathrm{C}$. Also displayed is the calculated sensitivity with the given $k_{\mathrm{ST}}$. 


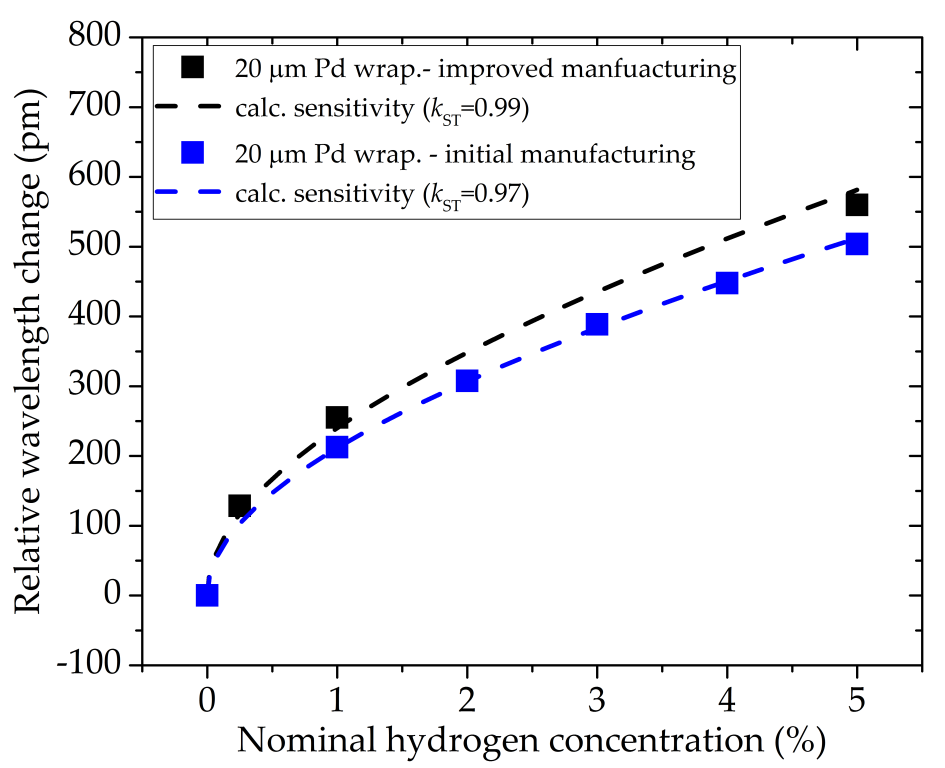

Figure 6.3: Comparison of the sensitivity with initial and improved manufacturing methods of the wrapped $20 \mu \mathrm{m}$ Pd foil sensors at $90^{\circ} \mathrm{C}$. Also displayed is the calculated sensitivity with the given $k_{\mathrm{ST}}$.

The hydrogen response of the two manufactured sensors is similar and within $3 \%$ of each other. This implies a high repeatability of the manufacturing process.

Compared to the initial manufacturing process the improved process with the manufacturing fixture increases the sensitivity by around 19\% (see Figure 6.3). The increase is not only due to the increased strain transfer coefficient but also due to the slightly different geometry of the sensor with more palladium in the circumference of the fiber (compare Figure 4.8 and Figure 4.7).

\subsection{The $100 \mu \mathrm{m}$ palladium foil sensor with improved manufacturing process}

Three $100 \mu \mathrm{m}$ palladium foil sensors were manufactured according to Section 4.2.3 using vacuum bagging and ADR 243 as an adhesive. One sensor broke during installation in the test chamber, and the remaining two sensors behaved similarly. 


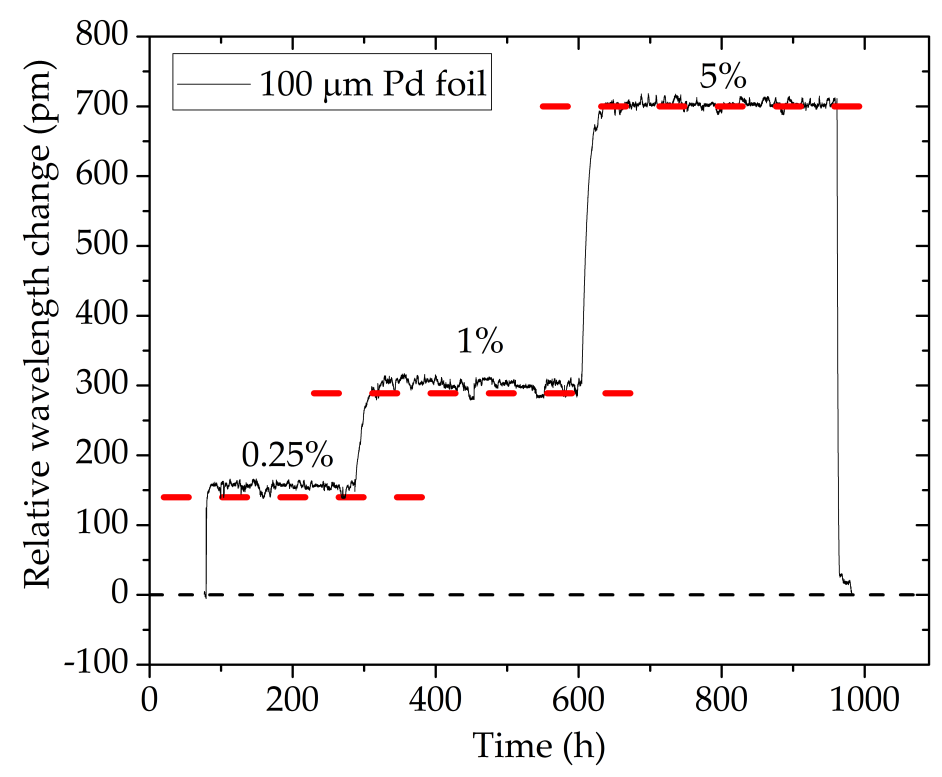

Figure 6.4: Sensor signal of a $100 \mu \mathrm{m} \mathrm{Pd}$ foil sensor at $90^{\circ} \mathrm{C}$ for three different hydrogen concentrations. Also displayed the calculated response for each concentration.

\subsubsection{Results}

The peak wavelength shifts of the sensor due to $0.25 \%, 1 \%$ and $5 \%$ hydrogen in nitrogen are shown in Figure 6.4. The final response to the steps is 155, 302, and $703 \mathrm{pm}$ respectively. After unloading, the offset from the starting wavelength was $+16 \mathrm{pm}$. In the following three $5 \%$ hydrogen cycles the wavelength change was repeatable with $687 \pm 3 \mathrm{ppm}$ and the response time was around 3 hours. The calculated peak wavelength shifts of the sensor due to $0.25 \%, 1 \%$ and $5 \%$ hydrogen were 140,289 , and 700 pm respectively. The deviation of the calculated response from the actual response is due to $k_{\mathrm{ST}} \neq 1$.

The sensitivity of both sensors is displayed in Figure 6.5. Also displayed is the calculated sensitivity with $k_{\mathrm{ST}}=0.99$. In the final three $5 \%$ hydrogen cycles the two sensors had repeatable response of $687 \pm 3 \mathrm{ppm}$ and $706 \pm 4 \mathrm{ppm}$. This leads to $k_{\mathrm{ST}}=0.98$ and $k_{\mathrm{ST}}=1.01$ respectively. The temperature sensitivity of the sensors was $22.3 \mathrm{pm} /{ }^{\circ} \mathrm{C}$ and $22.0 \mathrm{pm} /{ }^{\circ} \mathrm{C}$.

\subsubsection{Discussion}

The wavelength change of the sensor due to $1 \%$ hydrogen at $90^{\circ} \mathrm{C}$ is equivalent to the wavelength change caused by a temperature change of over $13^{\circ} \mathrm{C}$. This implies low temperature cross sensitivity and is an improvement of approxi- 


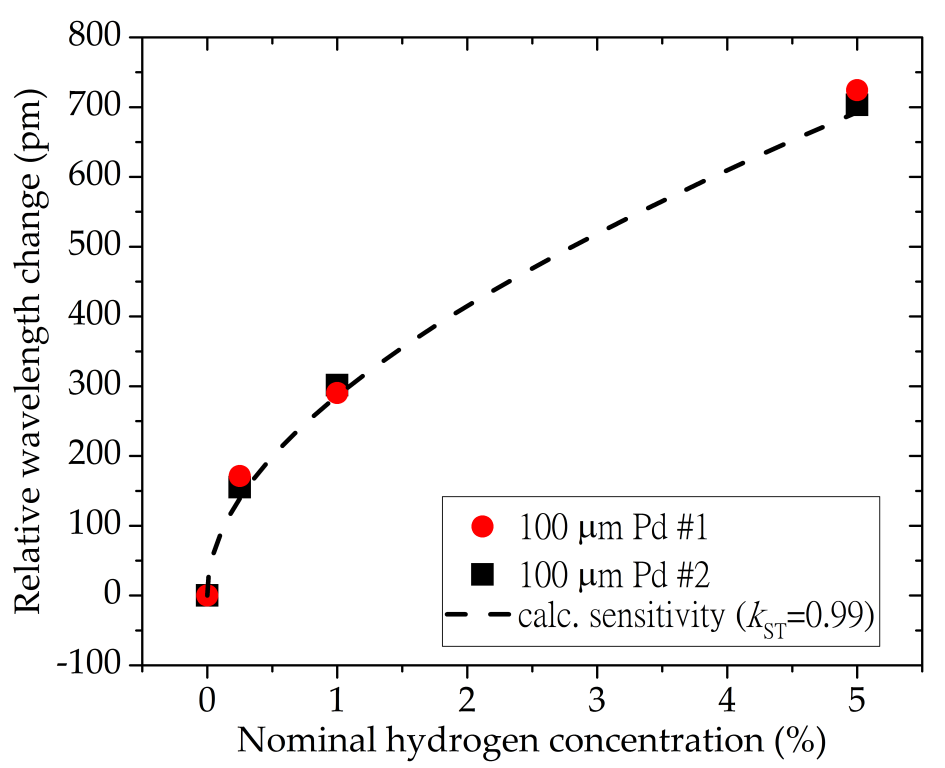

Figure 6.5: Sensitivity of the $100 \mu \mathrm{m}$ Pd foil sensors at $90^{\circ} \mathrm{C}$. Also displayed is the calculated sensitivity with the given $k_{\mathrm{ST}}$.

mately $96 \%$ over the sensor from the initial manufacturing process.

The hydrogen response of the two manufactured sensors is similar and within $3 \%$ of each other. This implies a high repeatability of the manufacturing process.

Compared to the initial manufacturing process the improved process with the vacuum bagging process increases the sensitivity by around $160 \%$ (see Figure 6.5). This significant increase might be partly due to a higher stiffness of the adhesive, but mainly due to the reduced bond line thickness that was achieved with the vacuum bagging method.

\subsection{The sandwiched $20 \mu \mathrm{m}$ palladium foil sensor}

Three sandwiched $20 \mu \mathrm{m}$ palladium foil sensors were manufactured according to Section 4.2.4 using vacuum bagging and ADR 243 as an adhesive. In this sensor design, a bare fiber is sandwiched in-between two $20 \mu \mathrm{m}$ palladium foils. All three sensors behaved similarly.

\subsubsection{Results}

The peak wavelength shifts of the sensor due to $0.25 \%, 1 \%$ and $5 \%$ hydrogen in nitrogen are shown in Figure 6.7. The final response to the steps is 145, 286, 


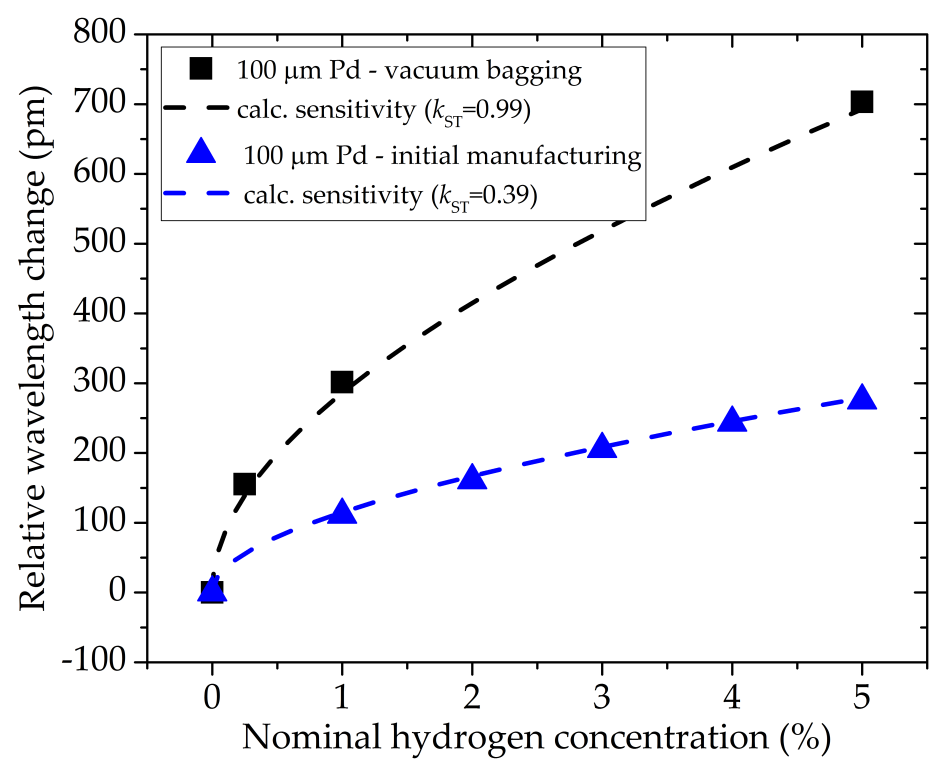

Figure 6.6: Comparison of the sensitivity with initial and improved manufacturing methods of the wrapped $100 \mu \mathrm{m}$ Pd foil sensors at $90^{\circ} \mathrm{C}$. Also displayed is the calculated sensitivity with the given $k_{\mathrm{ST}}$.

and 689 pm respectively. After reaching the maximum at the $5 \%$ step, the peak wavelength decreased by $28 \mathrm{pm}$ to $661 \mathrm{pm}$. After unloading, the offset from the starting wavelength was $-26 \mathrm{pm}$. In the following three $5 \%$ hydrogen cycles the wavelength change was repeatable with $689 \pm 2 \mathrm{ppm}$ and the response time was around 30 minutes. The calculated peak wavelength shift of the sensor due to $0.25 \%, 1 \%$ and $5 \%$ hydrogen is 134,275 , and 666 pm respectively. The deviation of the calculated response from the actual response is due to $k_{\mathrm{ST}} \neq 1$.

The sensitivity of the three sensors is displayed in Figure 6.8. Also displayed is the calculated sensitivity with a $k_{\mathrm{ST}}=1.06$. In the final three $5 \%$ hydrogen cycles the response of the two sensors was repeatable with $689 \pm 2$ ppm, $700 \pm 1$ and $714 \pm 2 \mathrm{ppm}$. This leads to $k_{\mathrm{ST}}=1.03, k_{\mathrm{ST}}=1.05$ and $k_{\mathrm{ST}}=1.07$ respectively(which is discussed in Section 6.4). The temperature sensitivity of the sensors was $23.2 \mathrm{pm} /{ }^{\circ} \mathrm{C}, 22.9 \mathrm{pm} /{ }^{\circ} \mathrm{C}$ and $23.3 \mathrm{pm} /{ }^{\circ} \mathrm{C}$.

\subsubsection{Discussion}

The wavelength change of the sensor due to $1 \%$ hydrogen at $90^{\circ} \mathrm{C}$ is equivalent to the wavelength change caused by a temperature change of over $12^{\circ} \mathrm{C}$. This implies low temperature cross sensitivity.

The hydrogen response of the three manufactured sensors is similar and within $4 \%$ of each other. This implies a high repeatability of the manufacturing 


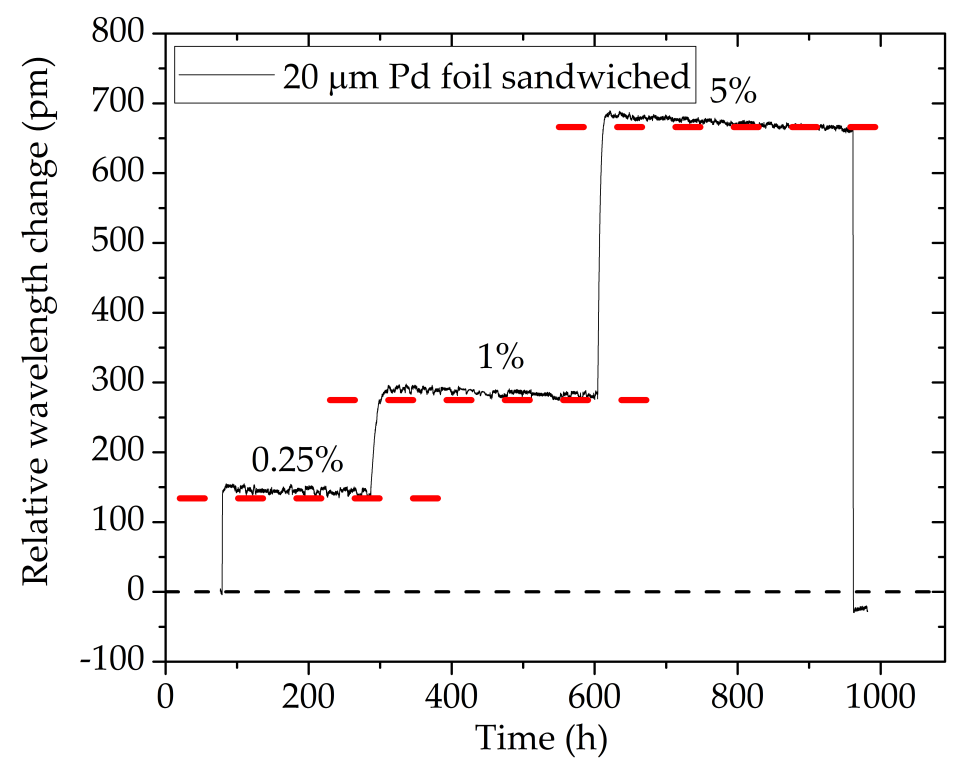

Figure 6.7: Sensor signal of a sandwiched $20 \mu \mathrm{m} \mathrm{Pd}$ foil sensor at $90^{\circ} \mathrm{C}$ for three different hydrogen concentrations. Also displayed is the calculated response for each concentration.

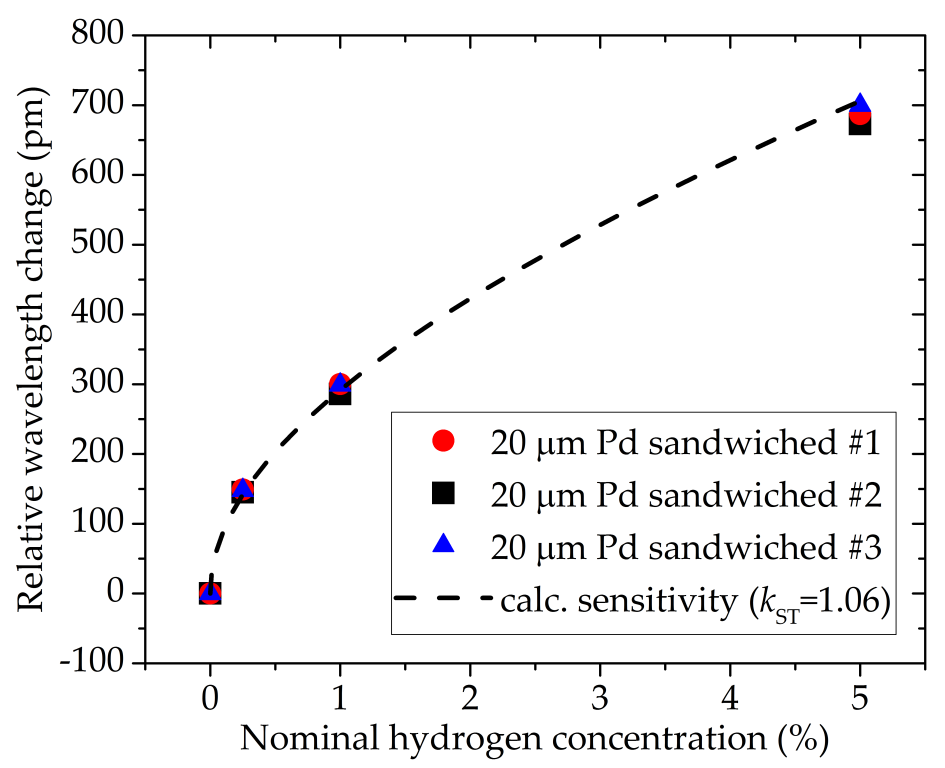

Figure 6.8: Sensitivity of the sandwiched $20 \mu \mathrm{m} \mathrm{Pd}$ foil sensors at $90^{\circ} \mathrm{C}$. Also displayed the calculated sensitivity with the given $k_{\mathrm{ST}}$. 
Table 6.1: Hydrogen and temperature sensitivity, resolution and accuracy of the foil sensors presented in this chapter. The hydrogen sensitivity, resolution, and accuracy was obtained for $1 \%$ hydrogen at $90{ }^{\circ} \mathrm{C}$. The temperature and hydrogen sensitivity are averaged over each group of sensors. The resolution and accuracy is obtained using the slope of the calculated sensitivity given in Figure 6.2, Figure 6.5, and Figure 6.8.

\begin{tabular}{lrrr}
\hline Sensitivity & wrapped $20 \mu \mathrm{m} \mathrm{Pd}$ & sandwiched $20 \mu \mathrm{m} \mathrm{Pd}$ & $100 \mu \mathrm{m} \mathrm{Pd}$ \\
\hline Hydrogen $\left(\mathrm{pm} / \% \mathrm{H}_{2}\right)$ & $253.5 \pm 1.5$ & $295.0 \pm 7.1$ & $295.9 \pm 5.7$ \\
Temperature $\left(\mathrm{pm} /{ }^{\circ} \mathrm{C}\right)$ & $21.2 \pm 0.4$ & $23.1 \pm 0.2$ & $22.2 \pm 0.1$ \\
\hline Resolution $\left(\mathrm{ppm} \mathrm{H}_{2}\right)$ & 81.0 & 67.0 & 64.4 \\
Accuracy $\left( \pm \mathrm{ppm} \mathrm{H}_{2}\right)$ & 243.0 & 200.9 & 193.1 \\
\hline
\end{tabular}

process.

\subsection{Comparison of the performance of the second generation foil sensors}

In this section we compare the results of the three different foil sensors presented in this chapter.

The sensitivity to hydrogen and temperature of all sensors (the values were averaged for each sensor type) is listed in Table 6.1. The hydrogen sensitivity was obtained for a $1 \%$ hydrogen step at $90^{\circ} \mathrm{C}$. All sensors showed a good hydrogen response with $253-295 \mathrm{pm} / \% \mathrm{H}_{2}$ and the sandwiched $\mu \mathrm{m}$ and the 100 $\mu \mathrm{m}$ palladium foil sensor particularly gave overall similar results. The temperature sensitivity of the sensors is similar and in the range of $21-23 \mathrm{pm} /{ }^{\circ} \mathrm{C}$.

The resolution and accuracy were calculated as described in Chapter 5. At $1 \%$ hydrogen and $90^{\circ} \mathrm{C}$ the resolution is around $81 \mathrm{ppm}$ hydrogen for the wrapped, and $67 \mathrm{ppm}$ hydrogen for the sandwiched $20 \mu \mathrm{m}$ palladium foil sensors. The $100 \mu \mathrm{m}$ palladium foil sensor has a resolution of around $64 \mathrm{ppm}$. At $1 \%$ hydrogen and $90^{\circ} \mathrm{C}$ the accuracy is around $\pm 243 \mathrm{ppm}$ hydrogen for the wrapped, and \pm 201 ppm hydrogen for sandwiched $20 \mu \mathrm{m}$ palladium foil sensors. The $100 \mu \mathrm{m}$ palladium foil sensor has a resolution of around $\pm 193 \mathrm{ppm}$.

All sensors presented in this chapter show excellent strain transfer between palladium foil and fiber with $k_{\mathrm{ST}} \geq 0.98$. Several sensors show an $k_{\mathrm{ST}}>1$, which by definition is not possible $\left(k_{\mathrm{ST}}\right.$ is defined as a value between 0 and 
1: at 0 no strain is transferred and at 1 all strain is transferred). However, several constants and parameters in the equations presented in Chapter 2 and Chapter 4 have uncertainties which can explain this phenomenon (e.g., the scaling factor $C$ alone has an uncertainty of around 5\%: see Section 4.1.2)

\subsection{Summary and conclusion}

All sensors presented in this chapter show excellent hydrogen response. With the new manufacturing tool the sensitivity of the wrapped $20 \mu \mathrm{m}$ palladium foil sensor could be increased by $19 \%$ and the manufacturing process proved to be reproducible. The vacuum bagging allowed even higher increase in sensitivity of around $160 \%$ for the $100 \mu \mathrm{m}$ palladium foil sensor. This manufacturing process also permitted reproducible manufacturing of sensors. With the vacuum bagging, a new sensor design, the sandwiched $20 \mu \mathrm{m}$ palladium foil sensors was manufactured and tested. These sensors also showed excellent hydrogen sensing behaviour.

An advantage of the $20 \mu \mathrm{m}$ compared to the $100 \mu \mathrm{m}$ palladium foil is the faster response time due to the shorter diffusion time in the bulk palladium. Another advantage is economic in nature as the decrease in thickness of the foil decreases the amount of palladium and therefore cost of the sensor.

The disadvantage of the $20 \mu \mathrm{m}$ palladium foil is the handling during manufacturing. The manufacturing of the wrapped sensor design requires a high level of finesse and is hard to scale with the current manufacturing fixture. The vacuum bagging of the $20 \mu \mathrm{m}$ palladium foil is more scalable. However, the alignment of the fiber and foil is not trivial, and significantly more difficult than it is for the $100 \mu \mathrm{m}$ palladium foil with the imprinted groove.

Since response time is not the main optimization parameter of the sensor, the $100 \mu \mathrm{m}$ palladium foil sensor manufactured with vacuum bagging is a promising solution for further development. The proven scalable manufacturing process with the vacuum bagging method is another beneficial and important factor for the commercial integration in a transformer. In Chapter 8 the $100 \mu \mathrm{m}$ palladium foil sensor is characterised for various hydrogen concentration at different temperatures in gas and oil environment. 


\section{Chapter 7}

\section{Amplification concepts for hydrogen sensors}

In this chapter the results of two sensing concepts for amplifying the sensitivity of palladium based hydrogen sensors are presented. The first sensor concept uses palladium silver alloy foil with increased hydrogen solubility compared to pure palladium. The second sensor concept is a novel design using a prestrained FBG and palladium foil, that concentrates the expansion of a longer palladium body on a shorter FBG.

The sensors in this section were tested simultaneously with the sensors in Chapter 6. During the experiment the test conditions were stable at $90.2 \pm 0.7^{\circ} \mathrm{C}$ and $1062 \pm 10$ mbar.

\subsection{The sandwiched $10 \mu \mathrm{m}$ palladium silver foil sen- sor}

Three sandwiched $10 \mu \mathrm{m}$ palladium silver foil sensors were manufactured according to Section 4.2 .5 using vacuum bagging and ADR 243 as an adhesive. A foil with $10 \mu \mathrm{m}$ thickness was chosen because of (a) the commercial availability and (b) the expected faster response time compared to thicker foils. This should decrease the effect of slower response time when using palladium silver instead of pure palladium for hydrogen sensing. The sandwiched design was chosen, because the manufacturing process proved to be repeatable and robust. One sensor broke during installation in the test chamber, and the remaining two sensors behaved similarly. 


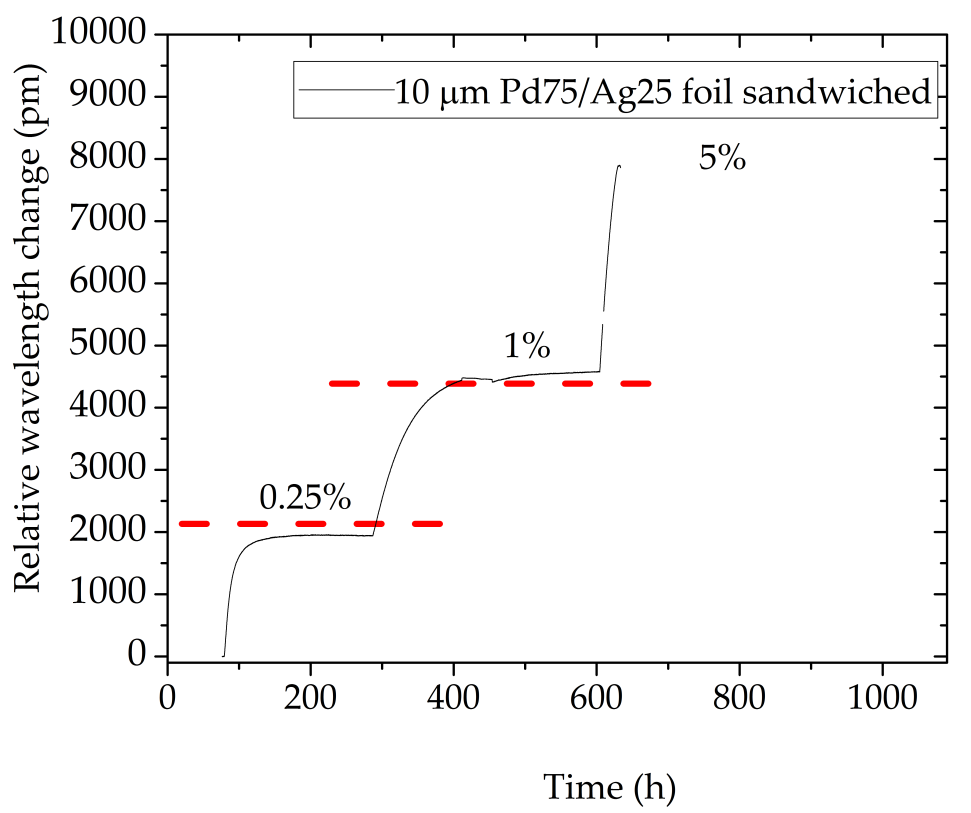

Figure 7.1: Sensor signal of a sandwiched $10 \mu \mathrm{m}$ palladium silver foil sensor at $90^{\circ} \mathrm{C}$ for three different hydrogen concentrations. Also displayed is the calculated response for each concentration.

\subsubsection{Results}

The peak wavelength shift of one sandwiched $\mu \mathrm{m}$ palladium silver foil sensor due to $0.25 \%, 1 \%$ and $5 \%$ hydrogen in nitrogen is shown in Figure 7.1. The final response to the steps is 1934, and $4553 \mathrm{pm}$ respectively. The response time was around 100 hours. During the increase from $1 \%$ to $5 \%$ the sensor was destroyed when the peak wavelength change was over $7.9 \mathrm{~nm}$. The extensive expansion of the palladium silver foil caused a partial delamination of the same from the FBG and as a consequence a peak split. The splitting of the peak is displayed in a spectral view over time in Figure 7.2. The calculated peak wavelength shift of the sensor due to $0.25 \%$, and $1 \%$ hydrogen is 2133 , and $4386 \mathrm{pm}$ respectively. The temperature sensitivity of the sensors was $22.6 \mathrm{pm} /{ }^{\circ} \mathrm{C}$, and $23.1 \mathrm{pm} /{ }^{\circ} \mathrm{C}$.

The sensitivity of both sensors is displayed in Figure 7.3. Also displayed is the calculated sensitivity with an amplification of $\varphi=17.6$.

\subsubsection{Discussion}

The wavelength change of the sensor due to $1 \%$ hydrogen at $90^{\circ} \mathrm{C}$ is equivalent to the wavelength change caused by a temperature change of over $83^{\circ} \mathrm{C}$. This implies an extremely low temperature cross-sensitivity.

The hydrogen response of the two manufactured sensors is similar and 


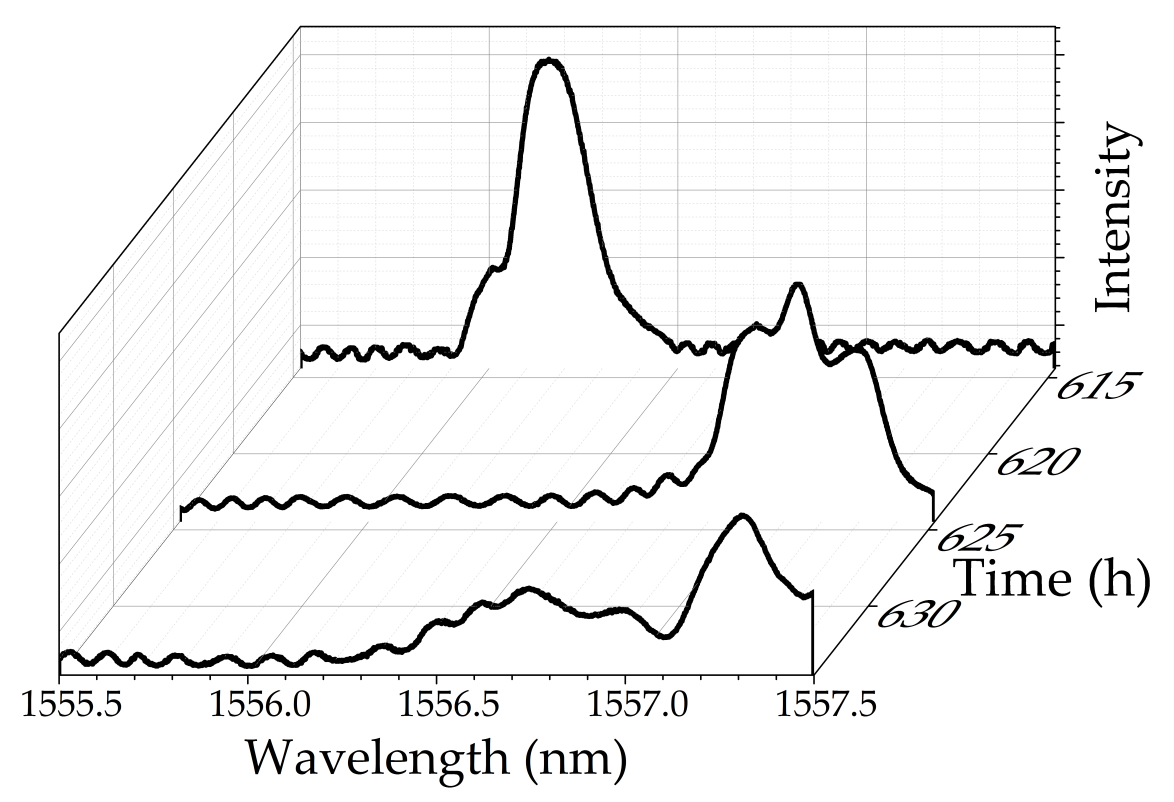

Figure 7.2: Peak splitting of the FBG as a sign of degradation of the bonding interface between palladium silver foil and fiber.

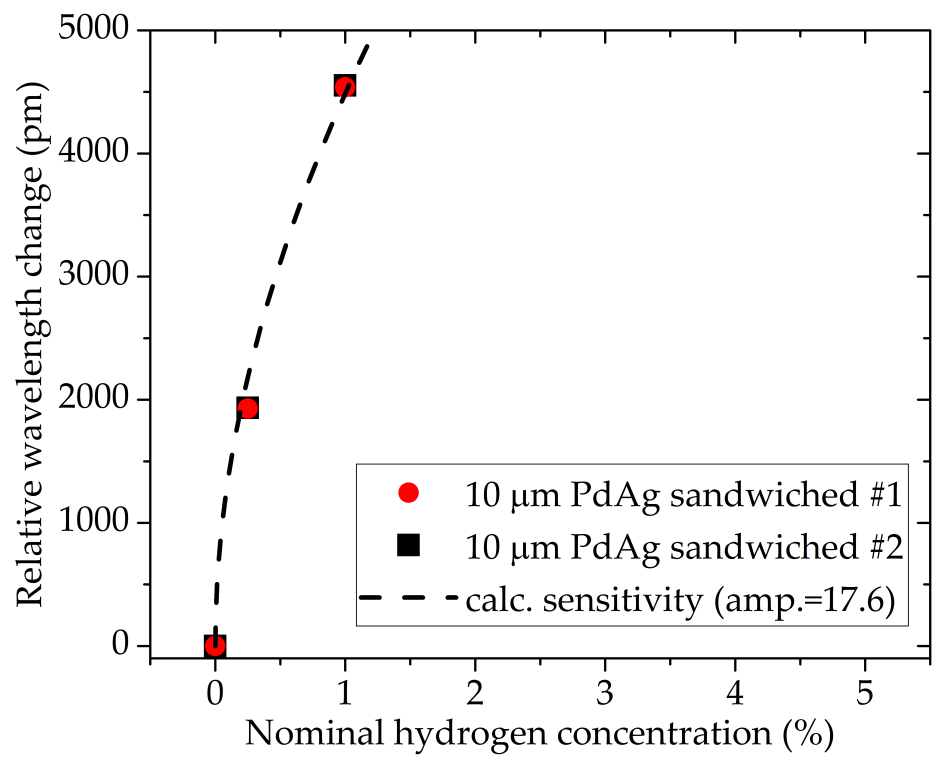

Figure 7.3: Sensitivity of the sandwiched $10 \mu \mathrm{m}$ palladium silver foil sensors at $90^{\circ} \mathrm{C}$. Also displayed is the calculated sensitivity using an amplification of 17.6 . 
within $0.3 \%$ of each other. This implies a high repeatability of the manufacturing process.

The palladium silver foil sensor response demonstrated significant amplification. The amplification of the $10 \mu \mathrm{m}$ foil using PdAg, compared to pure $\mathrm{Pd}$, is in the range of 17.6 (compare Figure 7.3). This amplification is larger, but close to the estimated amplification given in Section 4.2.5. However, the expansion of the foil was so extensive that it degraded the bonding interface and destroyed the sensor (compare 7.2) during the 5\% hydrogen step. Furthermore, the response time of the sensor is slow: over 4 days at $90^{\circ} \mathrm{C}$.

\subsection{The pre-strained sensor}

Three pre-strained sensors with $100 \mu \mathrm{m}$ palladium foil and Ormocer coated FBGs were manufactured according to Section 4.2.6. An Ormocer coated FBG was chosen, because of its improved mechanical stability. Compared to the coated and foil sensors, the sensitivity of the pre-strained sensor is not dependent on the thickness or coating material of the fiber. A mechanically stable sensor also facilitates the sensor manufacturing and increases longevity.

\subsubsection{Results}

The peak wavelength shift of one pre-strained sensor due to $0.25 \%, 1 \%$ and $5 \%$ hydrogen in nitrogen is shown in Figure 7.4. The final response to the steps is 460,852 , and 1815 pm respectively. After unloading, the offset from the starting wavelength was $158 \mathrm{pm}$. In the following three $5 \%$ hydrogen cycles the wavelength change was $1651 \pm 42 \mathrm{ppm}$ and the response time was around 2 hours. The calculated peak wavelength shifts of the sensor due to $0.25 \%, 1 \%$ and $5 \%$ hydrogen are 423,873 , and 2114 pm respectively. For the calculation an amplification of 2.92 was assumed (see Equation 4.4).

The palladium foil length was measured to be $a=76 \mathrm{~mm}$, the fiber length was $b=26 \mathrm{~mm}$, the bonding (fiber to $\mathrm{Pd}$ ) length with a thick adhesive layer on the palladium was $28 \mathrm{~mm}$ (see Figure 4.12). This results in a maximum amplification of 2.92 (if the expansion of the full length of the palladium relaxes the fiber and the adhesive layer does not influence the expansion of the $\mathrm{Pd}$ ), or in a minimum amplification of 1.85 (if the thick adhesive layer in the bonding area locally inhibits the expansion of the $\mathrm{Pd}$ ). The deviation of the calculated response from the actual response is due to the amplification $\varphi$ being less than 2.92. 


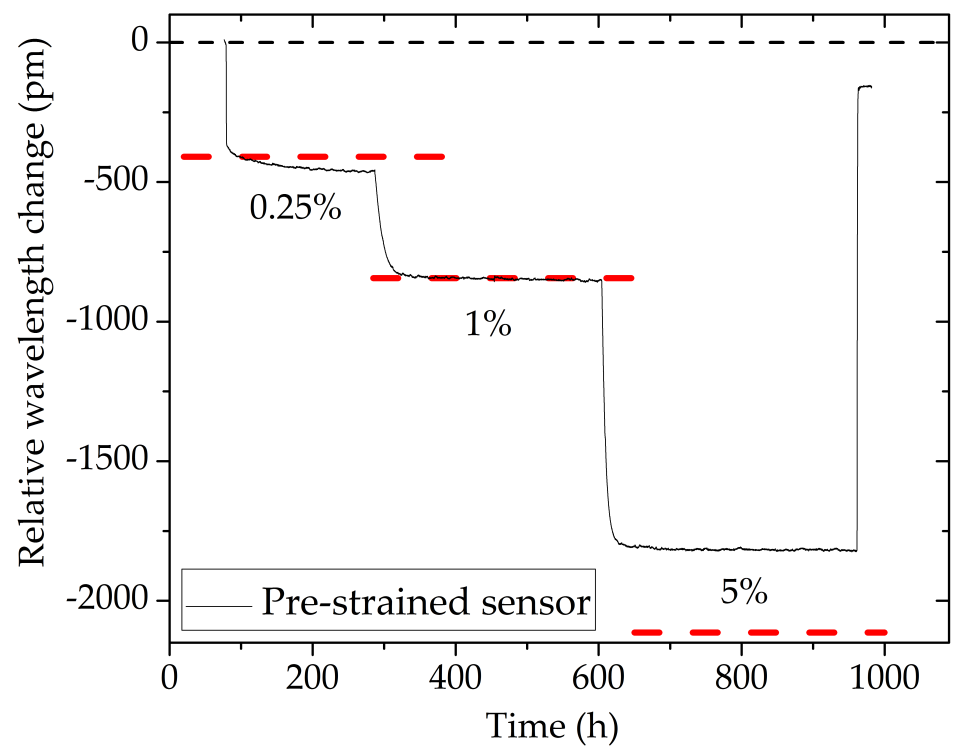

Figure 7.4: Sensor signal of a pre-strained palladium foil sensor at $90^{\circ} \mathrm{C}$ for three different hydrogen concentrations. Also displayed is the calculated response for each concentration.

The sensitivity of all three pre-strained sensors is displayed in Figure 7.5. Also displayed is the calculated sensitivity with $\varphi<2.5$.

In the final three $5 \%$ hydrogen cycles the response of the three sensors was $1463 \pm 2 \mathrm{pm}, 1651 \pm 42 \mathrm{pm}$, and $1606 \pm 5 \mathrm{pm}$. However, all pre-strained sensors showed an ongoing relaxation process over the entire measurement period (see Figure 7.6). The temperature sensitivity of the sensors was $15.4 \mathrm{pm} /{ }^{\circ} \mathrm{C}, 11.1$ $\mathrm{pm} /{ }^{\circ} \mathrm{C}$, and $22.3 \mathrm{pm} /{ }^{\circ} \mathrm{C}$.

\subsubsection{Discussion}

The wavelength change of the sensor due to $1 \%$ hydrogen at $90^{\circ} \mathrm{C}$ is equivalent tothe wavelength change caused by a temperature change of over $34^{\circ} \mathrm{C}$. This implies also a low temperature cross sensitivity.

The hydrogen sensitivity of the three pre-strained sensors is similar, and within $12.5 \%$ of each other. This comparably large deviation, can be explained by small variations in the length $a$, and $b$ and in the bond length of the adhesive interface.

The pre-strained sensor design demonstrated significant amplification. The amplification of the sensitivity is around 2.5 times the maximum calculated sensitivity of a palladium sensor (compare Figure 7.5). This is lower than the maximum estimated amplification of 2.92 and higher than the minimum es- 


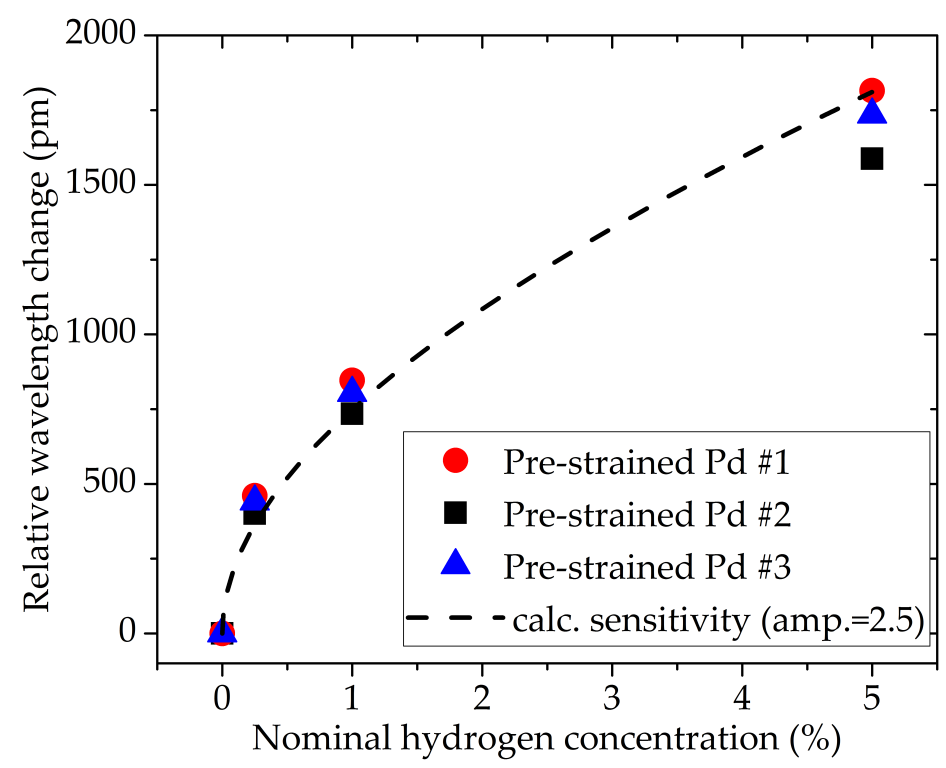

Figure 7.5: Sensitivity of the pre-strained palladium foil sensors at $90^{\circ} \mathrm{C}$. Also displayed is the calculated sensitivity with the given amplification of 2.5.

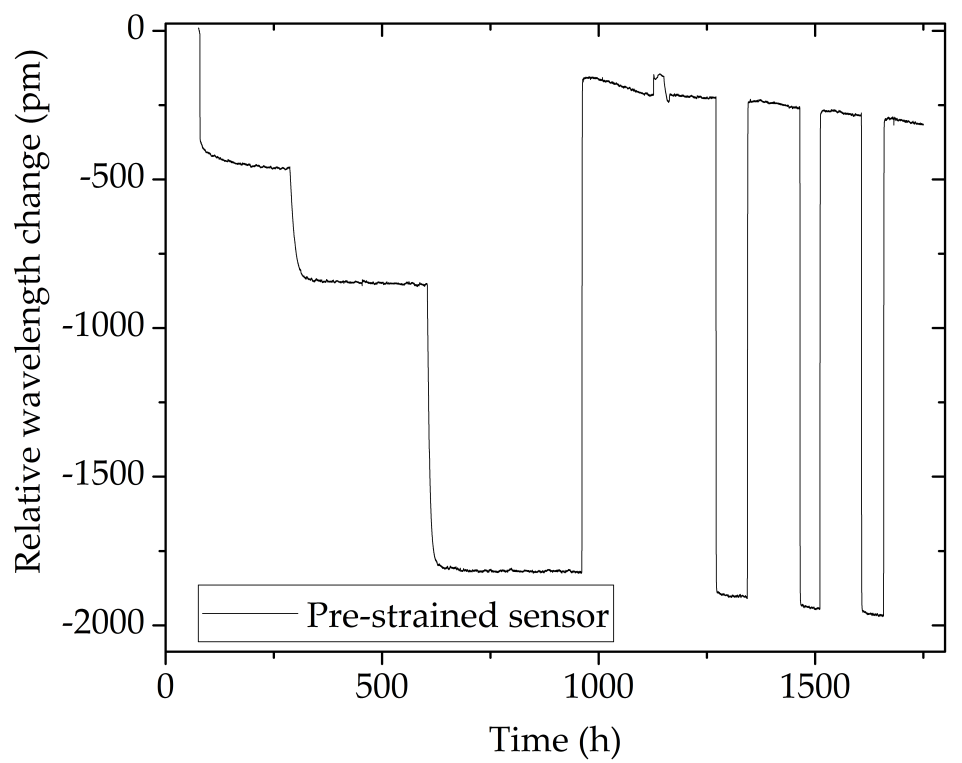

Figure 7.6: Hydrogen response of the pre-strained sensor over time. 
timated amplification 1.85. This could be due to the thick adhesive layer at the bonding interface of palladium and fiber inhibiting the expansion of the palladium locally. For the $0.25 \%$ and the $1 \%$ hydrogen steps, after the initial large response, a slow decrease of the peak wavelength is observed. One possible explanation is a relaxation process in the adhesive interface at the elevated temperature. Continued hydrogen cycling revealed a further relaxation. The overall relaxation was 320 pm after 68 days.

\subsection{Comparison}

In this section we compare the results of the two sensor designs with amplification presented in this chapter. In addition, the two sensors designs are compared with a non-amplified $100 \mu \mathrm{m}$ palladium foil sensor from Chapter 6 .

The sensitivity to hydrogen and temperature of the three sensors is listed in Table 7.1. The palladium silver sensor has the highest hydrogen sensitivity with $4545 \mathrm{pm} / \% \mathrm{H}_{2}$, followed by the pre-strained sensor with 5 times lower sensitivity of $795 \mathrm{pm} / \% \mathrm{H}_{2}$. The amplified sensors are 15 (palladium silver) and 2.5 (pre-strained) times more sensitive than the $100 \mu \mathrm{m}$ palladium foil sensor. The temperature sensitivity of all sensors is similar, with the pre-strained sensors having large variations among each other.

Comparing the sensitivity to literature values shows, that the sensitivity of our sensors is equal to or higher than that of other reported palladium based hydrogen sensors using FBG (without amplification) (compare Table 2.3). To our best knowledge, the sandwiched $10 \mu \mathrm{m}$ palladium silver foil sensor is the most sensitive palladium based hydrogen sensor using FBG reported so far.

The resolution and accuracy were determined as described in Chapter 5. At $1 \%$ hydrogen and $90^{\circ} \mathrm{C}$ the resolution is around $25 \mathrm{ppm}$ for the pre-strained sensor and $4 \mathrm{ppm}$ for the palladium silver sensor. Comparing these values with the resolution of the $100 \mu \mathrm{m}$ palladium foil sensor (64 ppm) highlight the increase due to the amplification. At $1 \%$ hydrogen and $90^{\circ} \mathrm{C}$ the accuracy is around $\pm 76 \mathrm{ppm}$ for the pre-strained and $\pm 13 \mathrm{ppm}$ for the palladium silver sensor.

Figure 7.7 shows the sensitivity of the sensors with amplification and of the $100 \mu \mathrm{m}$ palladium foil sensor. In addition, the theoretical maximum sensitivity of a sensor with an non-amplified, infinite palladium body attached to the fiber is displayed. The $100 \mu \mathrm{m}$ palladium foil sensor reaches $97 \%$ of the theoretical maximum sensitivity and with the pre-strained and palladium silver foil sensor 2.5 and 15 of the theoretical maximum sensitivity can be achieved. 
Table 7.1: Hydrogen and temperature sensitivity, resolution and accuracy of the sensor designs with amplification. For comparison the parameter for the $100 \mu \mathrm{m}$ sensor from Chapter 6 is listed as well. The hydrogen sensitivity was obtained for a $1 \%$ hydrogen step at $90{ }^{\circ} \mathrm{C}$. The resolution and accuracy was obtained for $1 \%$ hydrogen step at $90{ }^{\circ} \mathrm{C}$. ${ }^{*}$ The sensitivity for the pre-strained sensor might be lower due to the overlaying relaxation process.

\begin{tabular}{lrrr}
\hline Sensitivity & $100 \mu \mathrm{m}$ palladium & Pre-strained & sandwiched $10 \mu \mathrm{m} \mathrm{PdAg}$ \\
\hline Hydrogen $\left(\mathrm{pm} / \% \mathrm{H}_{2}\right)$ & $295.9 \pm 5.7$ & $795.0 \pm 55.9$ & $4545.3 \pm 7.6$ \\
Temperature $\left(\mathrm{pm} /{ }^{\circ} \mathrm{C}\right)$ & $22.2 \pm 0.1$ & $16.4 \pm 5.9$ & $22.9 \pm 0.3$ \\
\hline Resolution $\left(\mathrm{ppm} \mathrm{H}_{2}\right)$ & 64.4 & 25.4 & 4.2 \\
Accuracy $\left( \pm \mathrm{ppm} \mathrm{H}_{2}\right)$ & 193.1 & 76.1 & 12.7 \\
\hline
\end{tabular}

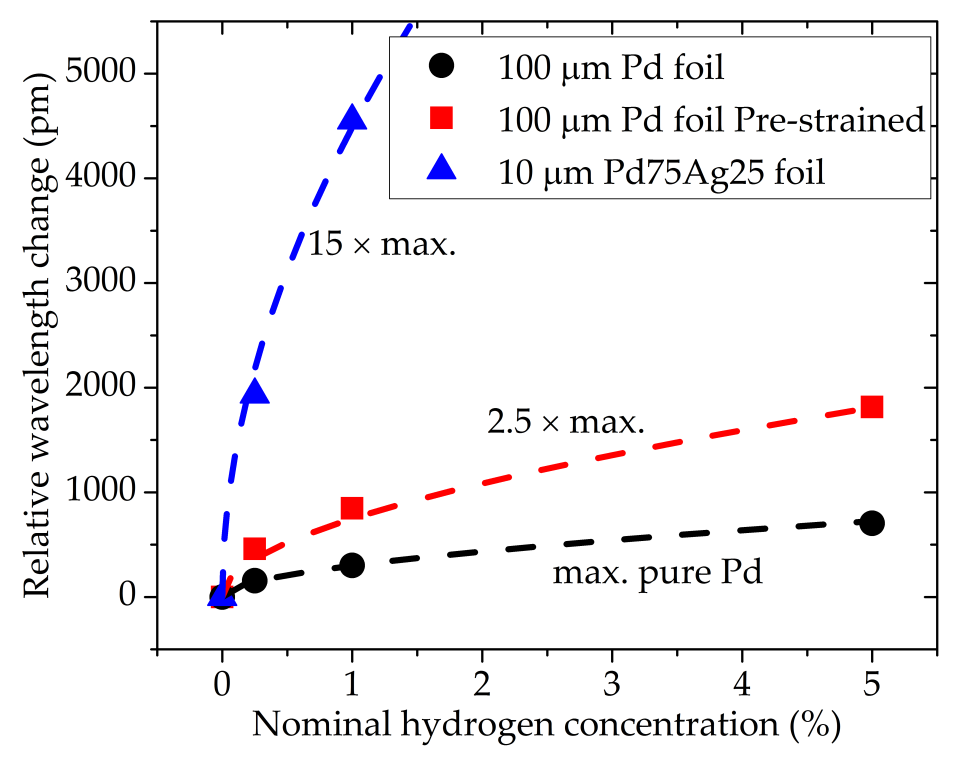

Figure 7.7: Comparison of the sensors with amplification to the non-amplified $100 \mu \mathrm{m}$ palladium foil sensor. Also displayed are the maximum response that is possible with a pure palladium based sensor, and this maximum response multiplied by a factor of 2.5 and, 15 . 


\subsection{Summary and conclusion}

A new concept using palladium silver foils to increase the hydrogen sensitivity compared to pure palladium foil was presented. By using a $10 \mu \mathrm{m}$ palladium silver foil the sensitivity could be increased by a factor of around 17 . The sensitivity of the palladium silver foil sensor is high with $4545 \mathrm{pm} / \% \mathrm{H}_{2}$ and it is therefore probably suitable to sense even below $1 \mathrm{ppm}$. With these values the sensor is the most sensitive palladium-based hydrogen sensor using FBG so far. However, this sensor concept would only suit low hydrogen concentrations $(\leqslant 1 \%)$ since the extensive expansion of the palladium silver foil can degrade the bonding interface between foil and fiber. 1\% hydrogen in the gas phase is equivalent to around 500 ppm dissolved hydrogen in oil (with an Ostwald coefficient of 0.05 ). Therefore, the $10 \mu \mathrm{m}$ palladium silver is unsuitable for the application in a transformer, since dissolved hydrogen concentration can exceed this value. Another disadvantage of the sensor is its slow response time. With 100 hours to reach a full response the sensor is not suitable for most applications (which require a faster response). Concerning the response time, improvements could be achieved with: (a) A reduced foil thickness to reduce the diffusion time. (b) A palladium coating on top of the palladium foil. By alloying silver to palladium the amount of palladium surface atoms is reduced significantly: Alloying 20\% silver reduces the surface palladium concentration to below 20\% [77]. This reduces the dissociation sites of the molecular hydrogen, since silver atoms do not have this capability. The reduced dissociation sites reduce the ad- and absorption process and therefore slow down the sensor response. By coating the palladium silver foil with palladium this effect might be mitigated.

Another novel concept using strain concentration and mechanical amplification of the hydrogen sensitivity with a pre-strained FBG and palladium foil was presented. With the pre-strained sensor the sensitivity could be increased by a factor of 2.5 and is high with $795 \mathrm{pm} / \% \mathrm{H}_{2}$. Compared to the palladium silver foil the amplification is not limited and only depends on the length ratio of palladium foil to FBG. With this sensing concept concentrations below 1 ppm can be measured. However, care must be taken that the expected expansion of the palladium does not exceed the pre-strain of the FBG, since the concept only works if the FBG is pre-strained. Furthermore, the palladium does not significantly contribute to the structural integrity of the sensor and therfore, can be chosen considerably thinner than other reported concept [95]). This leads to a faster response time and lower manufacturing costs. The sensitivity and response time therefore, can be tuned to the requirements of the 
application. However, before a practical application can be considered, the ongoing relaxation process (possibly due to creep in the adhesive interface) needs to be solved. Furthermore, the possible sensitivity to vibration, the sheer size and complexity of the sensor are likely cruxes for the practical application. These aspects rule the pre-strained sensor concept out for hydrogen monitoring in a transformer.

Both presented amplification concepts showed significant improvement in sensitivity. However, neither are suitable for the integration in a transformer: The palladium silver foil sensor responds slowly and degrades due to the large expansion at concentrations above $1 \%$ which is equivalent to only $500 \mathrm{ppm}$ in oil. The pre-strain sensor has a complex design and the observed relaxation would decrease the accuracy. Nevertheless, both concepts showed promising performance and will be subject to further investigation in the future. 


\section{Chapter 8}

\section{Characterisation in gas and oil}

In this chapter the results of the characterisation of the $100 \mu \mathrm{m}$ palladium foil sensors in gas and oil are presented and discussed. 15 sensors were manufactured with the vacuum bagging method described in 4.2.3. All sensors were manufactured and tested simultaneously. First the sensors were tested in gas at various temperatures and hydrogen concentrations. Then the 15 sensors were transferred to the oil test chamber and tested at $90^{\circ} \mathrm{C}$ and various hydrogen concentrations.

Additionally, the cross-sensitivity to carbon monoxide was investigated. Five $100 \mu \mathrm{m}$ palladium foil sensors, of which two had a $100 \mathrm{~nm}$ silicon oxide membrane (applied via sputter coating) on both sides of the foil, were manufactured with the vacuum bagging method. These sensors were tested at $90^{\circ} \mathrm{C}$ in nitrogen with $2.5 \%$ hydrogen and $5 \%$ carbon monoxide.

\subsection{Characterisation in gas}

First the sensors were tested in gas at $60^{\circ} \mathrm{C}, 75^{\circ} \mathrm{C}, 90^{\circ} \mathrm{C}, 105^{\circ} \mathrm{C}$, and $120^{\circ} \mathrm{C}$ (temperature accuracy $<0.2^{\circ} \mathrm{C}$ and stability $<0.15^{\circ} \mathrm{C}$ ). At each temperature the sensors were tested in different gas atmospheres at $1060 \pm 7 \mathrm{mbar}$ in the following order: Starting in nitrogen, the sensors were exposed to $5 \%$ hydrogen and then unloaded in air. This step was used to characterise the response time of the sensors. Afterwards, starting again in nitrogen, the sensors were tested using a hydrogen step increase at $0.25 \%, 0.5 \%, 1 \%, 2 \%$, and $5 \%$, before unloading them again in air. At $90^{\circ} \mathrm{C}$ the sensors were additionally tested at 0.01 (100 ppm), 0.05 (500 ppm), 0.1 (1000 ppm), 0.5\%, 3\%, and 4\%. Furthermore, after the second $5 \%$ step, the sensors were unloaded at $1 \%, 0.25 \%$ hydrogen, nitrogen and finally air to investigate the unloading behaviour and hysteresis. The response of one sensor to the whole testing sequence in gas at $90^{\circ} \mathrm{C}$ is shown in 


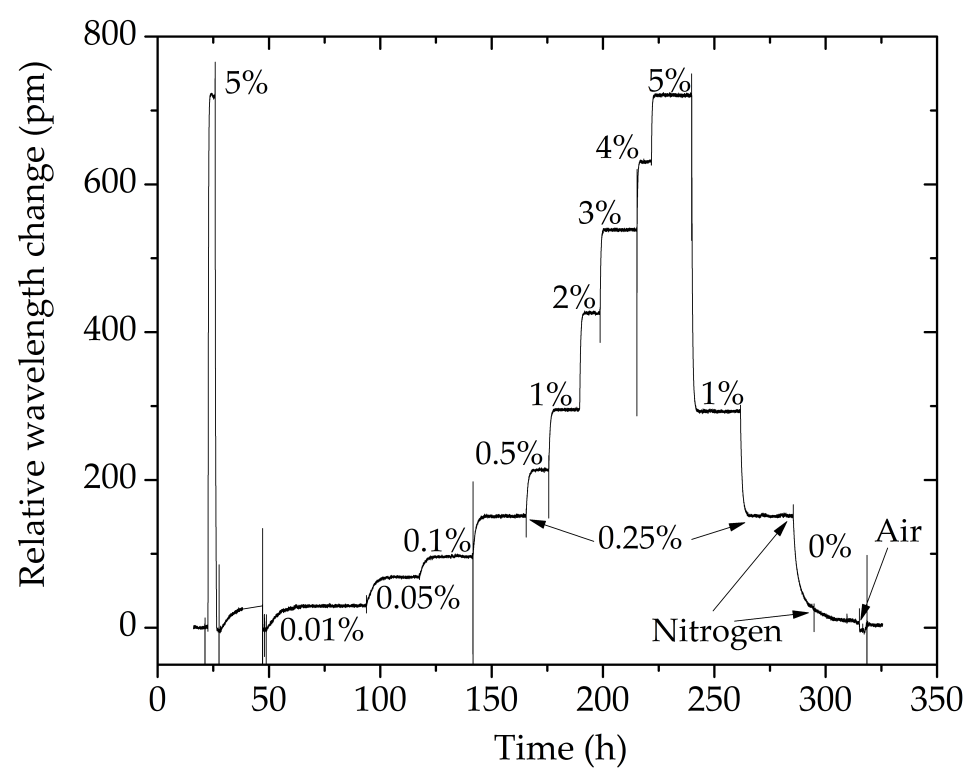

Figure 8.1: Response of a $100 \mu \mathrm{m}$ palladium foil sensor to various hydrogen concentrations at $90^{\circ} \mathrm{C}$.

Figure 8.1. The sharp spikes in the figure are caused by short temperature and flow rate instability during the change of the gas concentration. Furthermore, during the first $0.01 \%$ hydrogen step, the data acquisition was interrupted, and therefore, this step was repeated.

The relative peak wavelength change of one sensor is displayed in Figure 8.1. All 15 sensors behaved similarly. Comparing the wavelength change of the two $5 \%$ steps leads to a maximum deviation of $12 \mathrm{pm}$ for all 15 sensors at all tested temperatures, which indicates a precision of $< \pm 0.02 \%$ hydrogen. Comparing the wavelength change of loading and unloading at $90^{\circ} \mathrm{C}$ to $0.25 \%$ and $1 \%$ leads to a maximum deviation of $5 \mathrm{pm}$ and indicates a precision of $< \pm 0.04 \%$ hydrogen. Hence, no sign of hysteresis can be observed. During the final unloading step the nitrogen in the chamber was refreshed after 10 hours to reduce the amount of residual hydrogen (from mixing and in the palladium). After 20 more hours the atmosphere was changed to dry air, to fully unload the sensors. After introducing air, a wavelength change of around 10 pm was measured.

In Figure 8.2 the hydrogen sensitivity of one sensor at different temperatures is displayed. Next to the experimental results, the calculated sensitivity is shown. The sensitivity was calculated with an individual $k_{\mathrm{ST}}$ for each temperature. $k_{\mathrm{ST}}$ was determined from the $5 \%$ response. For the displayed sensor the coefficient of strain transfer is $k_{\mathrm{ST}}=1.03 \pm 0.01$. 


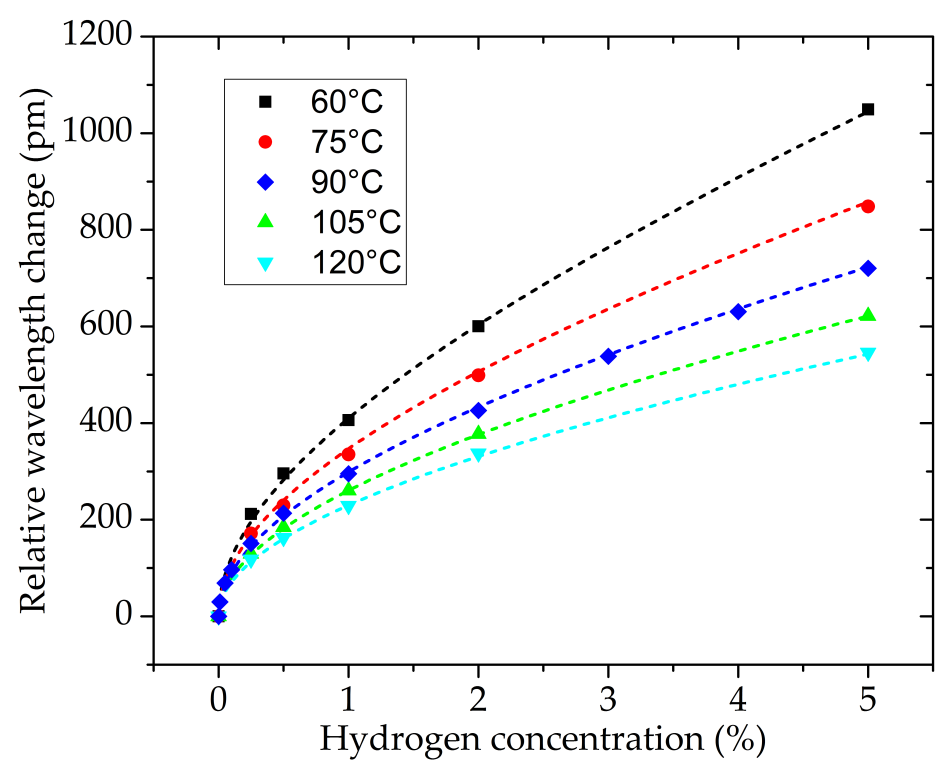

Figure 8.2: Hydrogen sensitivity of a $100 \mu \mathrm{m}$ palladium foil at various temperatures. Also displayed the predicted sensitivity (dashed lines) of the sensor determined with $k_{\mathrm{ST}}=1.03 \pm 0.01$.

\subsubsection{Response time}

The response time is also analysed. The response time was calculated by using the parameters from an exponential decay fit and defined as the time when $99 \%$ of the final wavelength change is reached. The average response time of all 15 sensors is displayed in Figure 8.3. For a 5\% hydrogen step the response time is on average 330 minutes at $60^{\circ} \mathrm{C}$, decreases to 230 minutes at $75^{\circ} \mathrm{C}$, and further to 83 minutes at $90^{\circ} \mathrm{C}$. At $105^{\circ} \mathrm{C}$ and $120^{\circ} \mathrm{C}$ the response time is on average 87 and 71 minutes respectively. As the box percentiles and errorbars indicate, the response time varies strongly and some sensors respond significantly faster, or slower.

\subsubsection{Carbon monoxide cross-sensitivity}

In this section the cross-sensitivity of the sensors to carbon monoxide is investigated. Carbon monoxide has been shown to inhibit the hydrogen absorption and thus slow the sensor response, or even to lead to an unresponsive sensor (see Section 2.2.7). The cross-sensitivity experiment was performed in test setup II. During the experiment the pressure was $1059 \pm 10$ mbar and the temperature was $90 \pm 1.5^{\circ} \mathrm{C}$. The higher uncertainty in pressure and temperature compared to the experiments performed in setup I is due to room temperature 


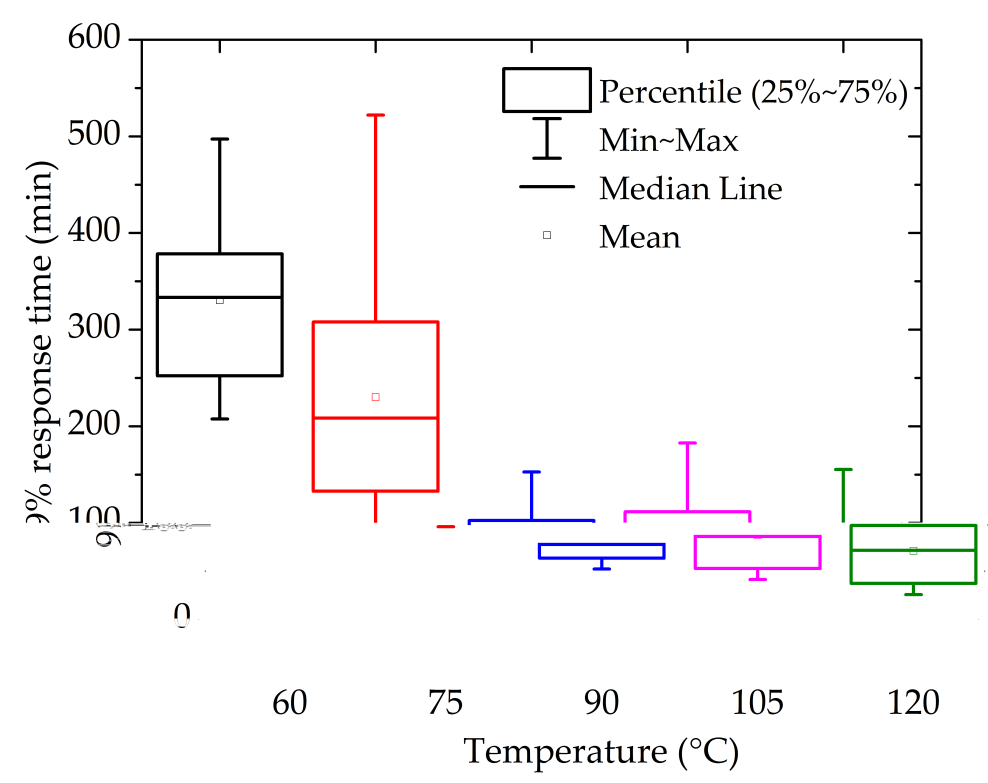

Figure 8.3: Average response time of the 15 manufactured sensors.

fluctuations in the laboratory the setup was placed in. The FBGs were interrogated using the Micronoptics si155 interrogator. Compared to the Anritsu OSA it has a lower resolution resulting in a noisier signal. Three $100 \mu \mathrm{m}$ palladium foil sensors and two $100 \mu \mathrm{m}$ palladium foil sensors with silicon dioxide membrane were tested.

The loading behaviour starting from nitrogen atmosphere was tested in nitrogen atmosphere with $2.5 \%$ hydrogen, or $2.5 \%$ hydrogen and $5 \%$ carbon monoxide.

The unloading behaviour starting from $2.5 \%$ hydrogen was tested in dry air, nitrogen, or $5 \%$ carbon monoxide in nitrogen.

The response time of one $100 \mu \mathrm{m}$ palladium foil sensor to $2.5 \%$ hydrogen with and without the presence of carbon monoxide is shown in Figure 8.4. The other $100 \mu \mathrm{m}$ palladium foil sensors behaved similarly. The response time without the presence of carbon monoxide is around 30-140 minutes. The response time with carbon monoxide is significantly higher with around 43-56 hours.

The sensors were unloaded in dry air, nitrogen, or in nitrogen with 5\% carbon monoxide. The unloading behaviour of the sensor is displayed in Figure 8.5. In air the sensors fully unload in around 60 minutes, in nitrogen the sensors unload in around 40 hours with a final offset of around $60 \mathrm{pm}$. Of the $60 \mathrm{pm}$, $30 \mathrm{pm}$ can be explained by a lower starting peak wavelength for the nitrogen and carbon monoxide unloading. The other $30 \mathrm{pm}$ can be explained 


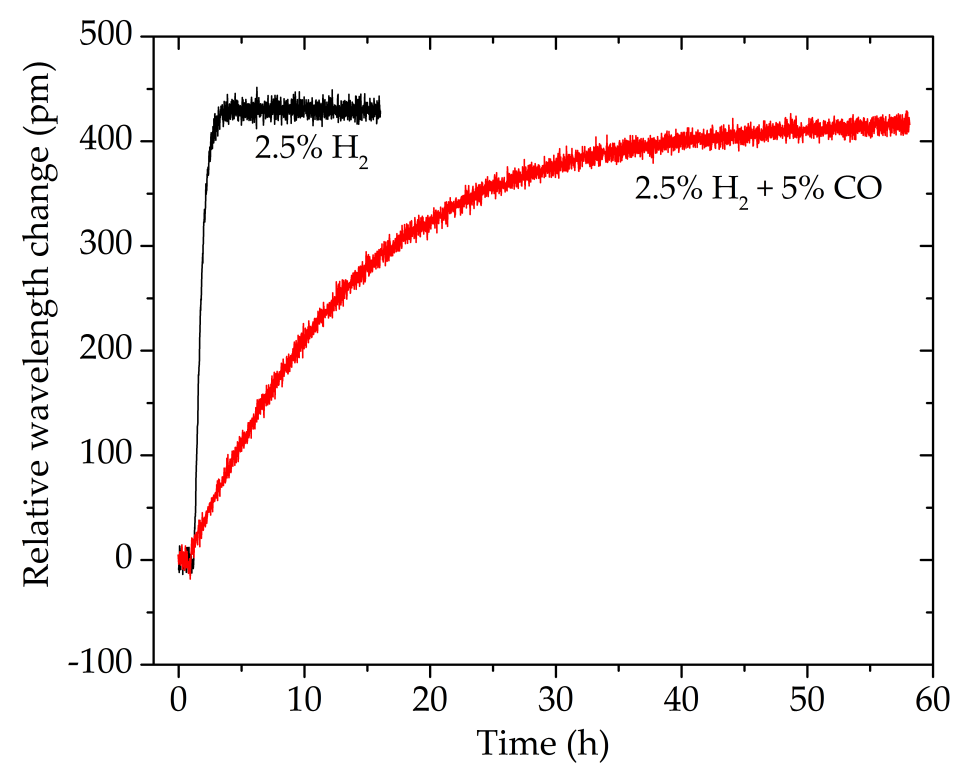

Figure 8.4: Response of a $100 \mu \mathrm{m}$ palladium foil sensor to $2.5 \%$ hydrogen at $90^{\circ} \mathrm{C}$ with and without the presence of carbon monoxide.

with residual hydrogen in the test setup. When unloading with air, the residual hydrogen is combusted (with oxygen) resulting in a fully unloaded sensor. When unloading in atmosphere without oxygen, residual hydrogen remains in the setup. The sensors could be fully unloaded ( $30 \mathrm{pm})$ by exposure to dry air. In 5\% carbon monoxide the sensors unload in around 300 hours. The sensors also did not fully unload and an offset of around $60 \mathrm{pm}$ remained. Afterwards, the sensors could be also fully unloaded in dry air.

Thin silicon dioxide films improved the response time of palladium based thin film hydrogen sensors in other carbon monoxide cross-sensitivity experiments [69]. A $100 \mathrm{~nm}$ silicon oxide membrane was sputter coated on both sides of the palladium foil before attaching it to the fiber. The response of a sensor (with and without membrane) to $2.5 \%$ hydrogen is displayed in Figure 8.6. The response time of the sensor with membrane is around 8-12 hours and therefore significantly slower than the sensor without membrane.

The response of the sensor (with and without membrane) to $2.5 \%$ hydrogen and 5\% carbon monoxide is displayed in Figure 8.7. No significant difference in response time can be observed when carbon monoxide is present.

Concerning the unloading behaviour, the sensors with membrane showed slower unloading in air (around 4 times slower, see Figure 8.8) and nitrogen (around 2 times slower, see Figure 8.9) compared to the sensors without membrane. In the presence of carbon monoxide however, the sensors with mem- 


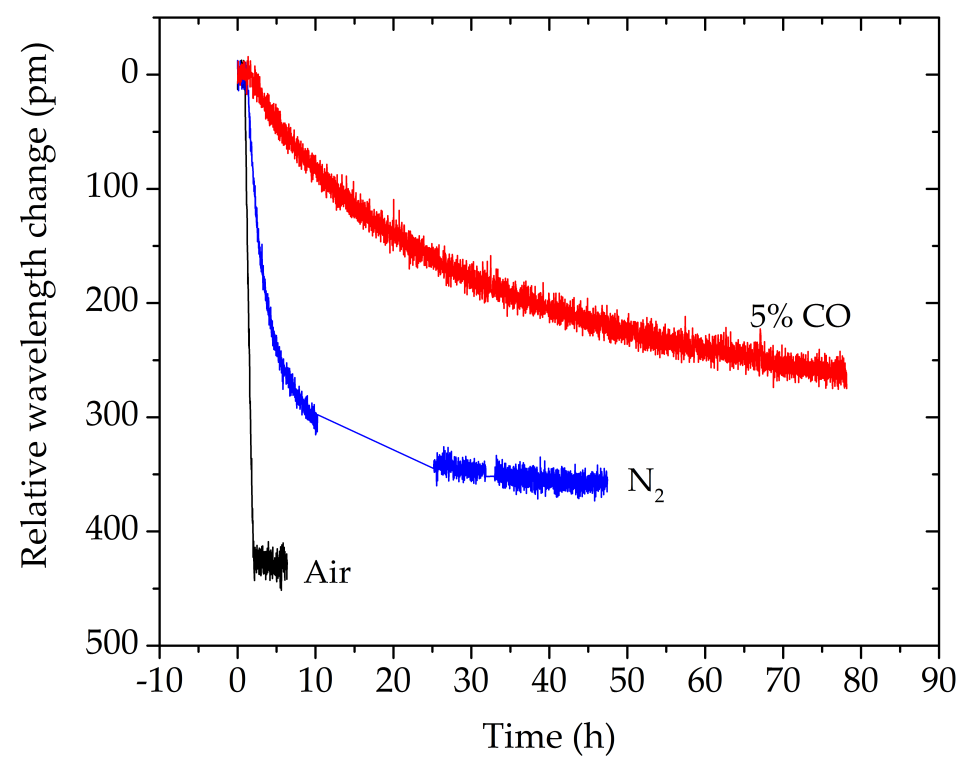

Figure 8.5: Unloading of a $100 \mu \mathrm{m}$ palladium foil sensor from $2.5 \%$ hydrogen at $90^{\circ} \mathrm{C}$ in different gas atmospheres. During the nitrogen unloading the data acquisition was interrupted between hour 10 and 25 .

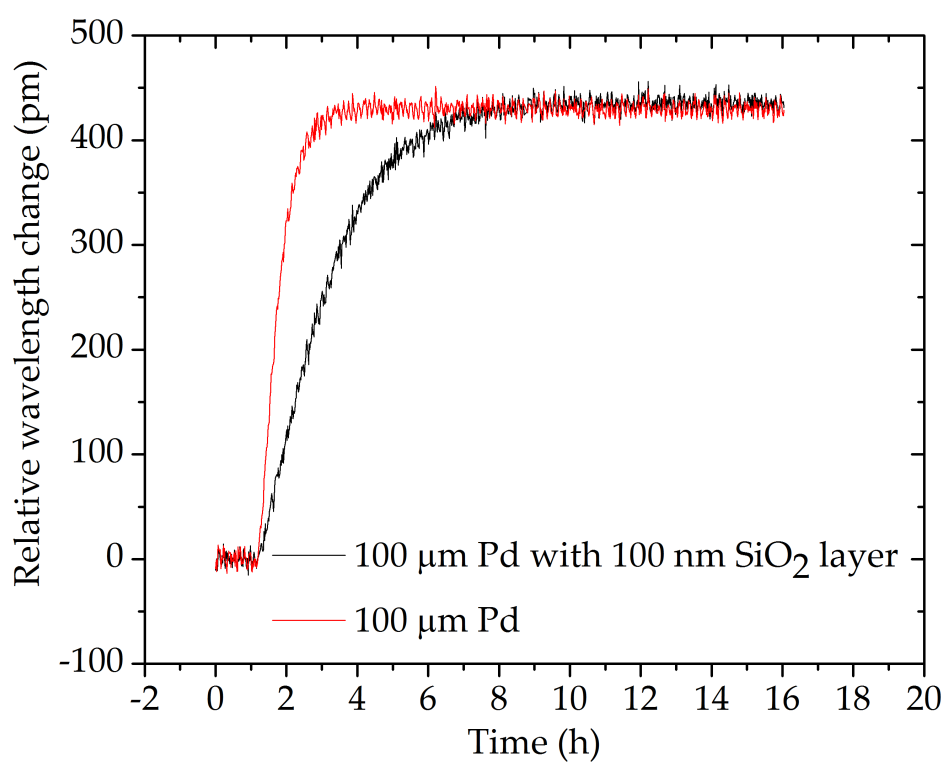

Figure 8.6: Response of a $100 \mu \mathrm{m}$ palladium foil sensor with and without 100 $\mathrm{nm}$ silicon oxide membrane to $2.5 \%$ hydrogen at $90^{\circ} \mathrm{C}$. 


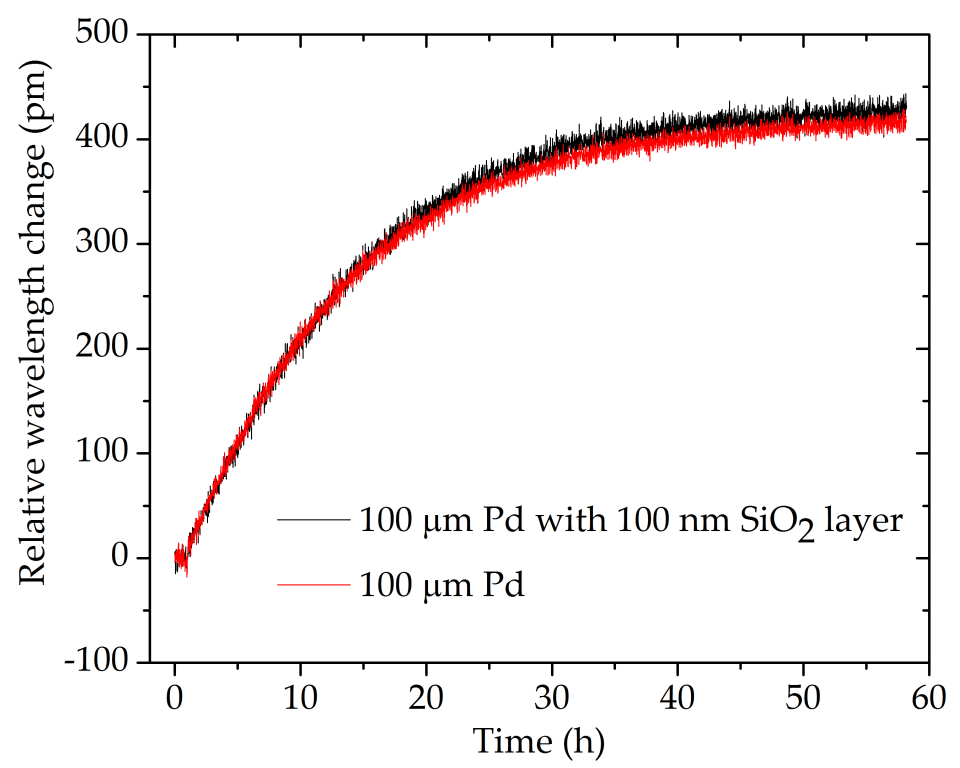

Figure 8.7: Response of a $100 \mu \mathrm{m}$ palladium foil sensor with and without 100 $\mathrm{nm}$ silicon oxide membrane to $2.5 \%$ hydrogen in the presence of $5 \%$ carbon monoxide at $90^{\circ} \mathrm{C}$.

brane showed faster unloading in the presence of carbon monoxide (around 2 times faster, see Figure 8.10) compared to the sensors without membrane.

\subsubsection{Discussion}

15 sensors were manufactured in one batch using the vacuum bagging method. All 15 sensors showed similar hydrogen sensitivity. $k_{\mathrm{ST}}$ derived from a $5 \%$ hydrogen measurement at the five different temperatures is in the range of $1.03 \pm 0.03$ for all sensors. This implies that the manufacturing methods with vacuum bagging allow high repeatability between the sensors. The temperature dependence of the hydrogen sensitivity obtained for a $1 \%$ hydrogen step is displayed in Figure 8.11. The average hydrogen sensitivity of all 15 sensors is $407 \mathrm{pm}, 339 \mathrm{pm}, 297 \mathrm{pm}, 258 \mathrm{pm}$, and $234 \mathrm{pm}$ at $60^{\circ} \mathrm{C}, 75^{\circ} \mathrm{C}, 90^{\circ} \mathrm{C}, 105^{\circ} \mathrm{C}$, and $120^{\circ} \mathrm{C}$ respectively.

The precision of the individual sensors was investigated by two (loading) $5 \%$ hydrogen steps at all temperatures and additionally by two (loading, and unloading) $0.25 \%$ and $1 \%$ hydrogen steps at $90^{\circ} \mathrm{C}$. Overall the precision of the 15 sensors is $< \pm 0.04 \%$ hydrogen.

The response time decreases significantly with increasing temperature from 330 minutes at $60^{\circ} \mathrm{C}$ to 83 minutes at $90^{\circ} \mathrm{C}$. The difference in response time at $90^{\circ} \mathrm{C}, 105^{\circ} \mathrm{C}$, and $120^{\circ} \mathrm{C}$ is small. 


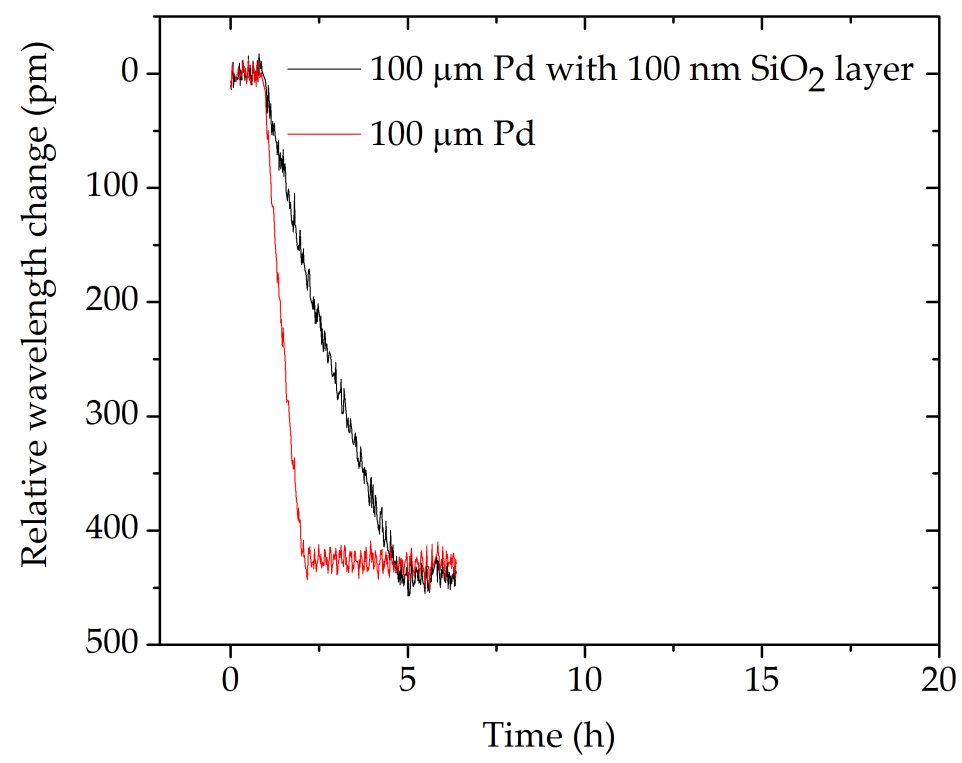

Figure 8.8: Unloading of a $100 \mu \mathrm{m}$ palladium foil sensor with and without 100 nm silicon oxide membrane in air at $90^{\circ} \mathrm{C}$.

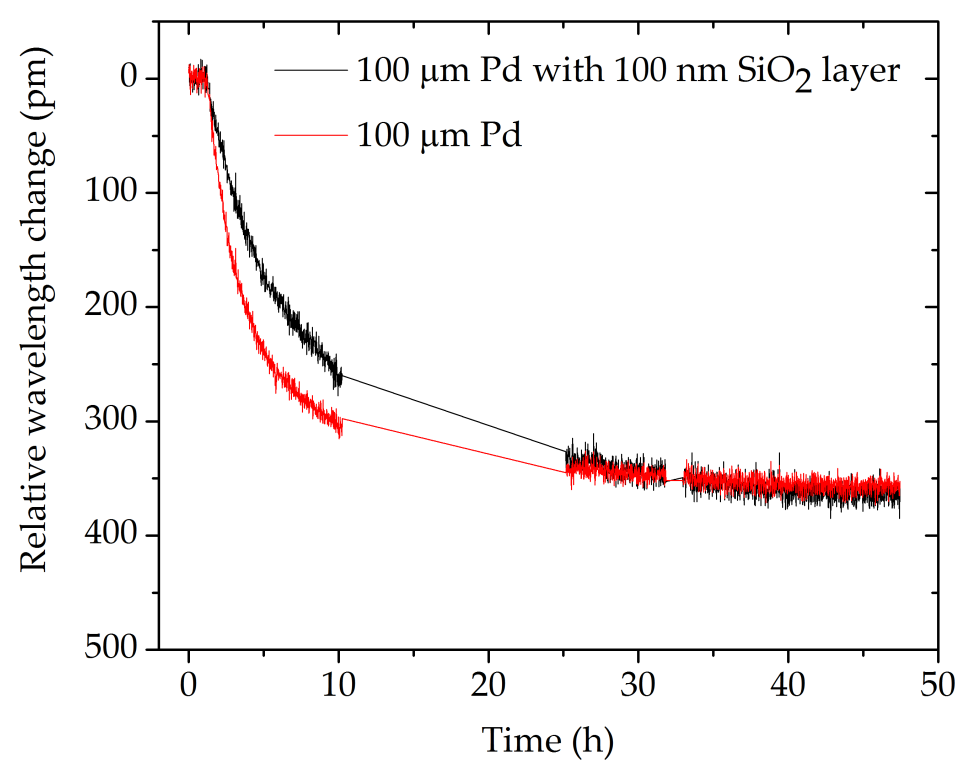

Figure 8.9: Unloading of a $100 \mu \mathrm{m}$ palladium foil sensor with and without 100 $\mathrm{nm}$ silicon oxide membrane in nitrogen at $90^{\circ} \mathrm{C}$. During the nitrogen unloading the data acquisition was interrupted between hour 10 and 25 . 


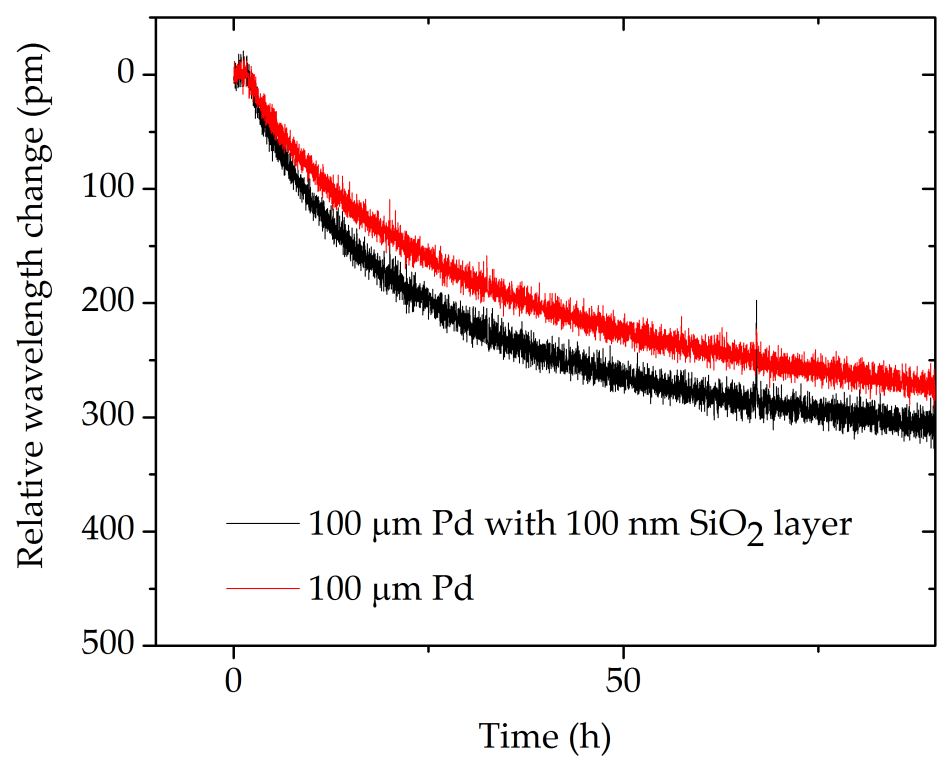

Figure 8.10: Unloading of a $100 \mu \mathrm{m}$ palladium foil sensor with and without 100 nm silicon oxide membrane in a gas mixture of $95 \%$ nitrogen and $5 \%$ carbon monoxide at $90^{\circ} \mathrm{C}$.

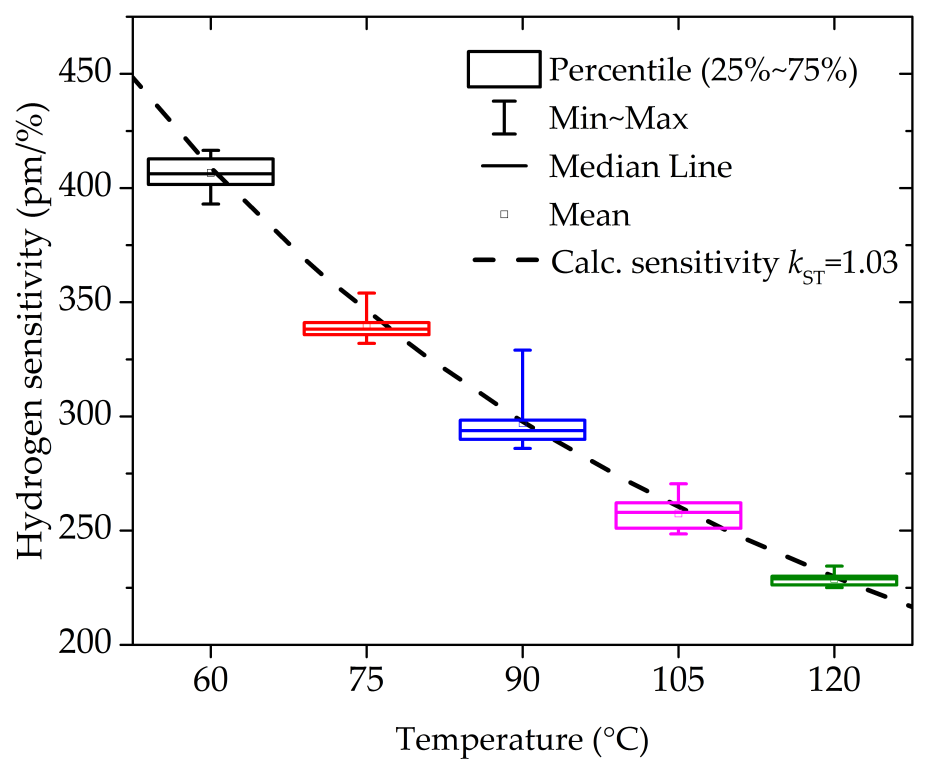

Figure 8.11: Average hydrogen sensitivity of all 15 manufactured sensors at various temperatures. Also displayed, the calculated hydrogen sensitivity (dashed line). 
The response times of the individual sensors differ quite strongly (see Figure 8.3). A possible explanation is surface contamination (e.g., excess adhesive during manufacturing forming a membrane on the palladium) which slows the hydrogen absorption in the palladium. The variability in response time of the individual sensors correlates well with the assumption that the dissociation process and not the diffusion process is the rate determining step of the absorption process. If diffusion was the rate determining step, the response time of the 15 sensors would be expected to be the same, as they are all made of the same palladium foil (material and geometry). Furthermore, if diffusion was the rate determining step a significantly faster response would be expected, as the calculated diffusion time is around 30 seconds at $90^{\circ} \mathrm{C}$ (see Figure 2.1). In contrary, the experimental response time of the sensors is in good agreement with the calculated response time following equation (2.9), which assumes the dissociation to be the rate determining step (see Figure 2.2). The calculated response time using equation (2.9) is around 20 minutes at $90^{\circ} \mathrm{C}$ and therefore in better agreement with the response times of the sensors determined in the experiments. The deviation of the calculated from the experimental response times can be again explained by surface contamination of the palladium.

The cross-sensitivity showed that that the hydrogen response is slowed in the presence of carbon monoxide. However, the carbon monoxide did not lead to an unresponsive sensor as reported by [51], nor had an impact on the magnitude of the signal. Applying a silicon oxide membrane to reduce the effect of carbon monoxide showed mixed results. The response time to loading in hydrogen and unloading in air and nitrogen is slowed due to the membrane. There was no significant difference in the response time of loading in hydrogen with the presence of carbon monoxide. However, the membrane improved the response time when unloading in the presence of carbon monoxide.

\subsection{Characterisation in oil}

After testing in gas, the 15 foil sensors were transferred to the oil test chamber. Testing was performed at $90^{\circ} \mathrm{C}$ and gas mixtures with the same hydrogen concentrations as in the gas measurements $(0.01,0.05,0.1,0.25,0.5,1,2,3,4,5 \%$ hydrogen) were bubbled through the oil. The testing was performed in two segments separated by a 3 week break in which the sensors were stored in the oil, at room temperature and nitrogen. In the first segment the sensors were tested from 0.01 to $1 \%$ hydrogen (see Figure 8.12) and in the second segment from 2 to $5 \%$ hydrogen (see Figure 8.13). The usability concerning the preparation of the gas mixtures of setup 2 is inferior to setup 1 . The accessibility of 


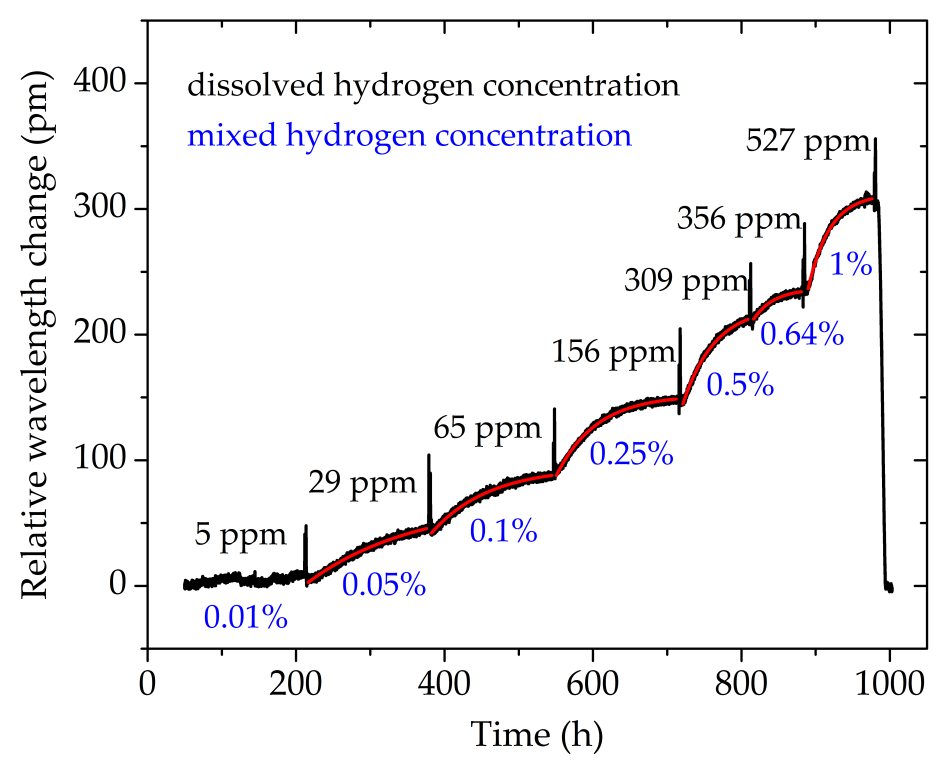

Figure 8.12: Response of a $100 \mu \mathrm{m}$ palladium foil sensor (first segment). The exponential decay fit is also displayed (red). The hydrogen concentrations are given in \% for prepared gas mixture (red) and in ppm for the measured, dissolved hydrogen concentration (blue).

the valves is limited, since the setup 2 is placed in a fume hood. As a result the gas mixtures of the first $1 \%$ and the $3 \%$ hydrogen step were unintentionally prepared to be $0.64 \%$ and $2.22 \%$. After the first segment, the sensors were unloaded by bubbling air. In the second segment the circulation pump failed (diaphragm ruptured) during the last (5\%) hydrogen step. A replacement pump (same model) was installed and the experiment was continued. However, the replacement pump caused several issues: the replacement pump had a higher throughput. This caused an increased overpressure between the pump and subsequent flow meter, which caused leakage in the connector between pump and system. In addition, the FBG signal noise increased when the replacement pump was running, probably also due to increased and unstable flow (see Figure 8.13). Therefore, the pump was turned off after installation until the hydrogen sensors were saturated. The effect of the pump being turned off, should only be a slower diffusion of hydrogen from gas into oil, and thus a slowed response of the sensor. For the unloading in air, the pump was turned on, since the leakage would have only affected a hydrogen measurement. After the sensors were fully unloaded the pump was again turned off to reduce the signal noise.

The relative peak wavelength change of one sensor is displayed in Figure 


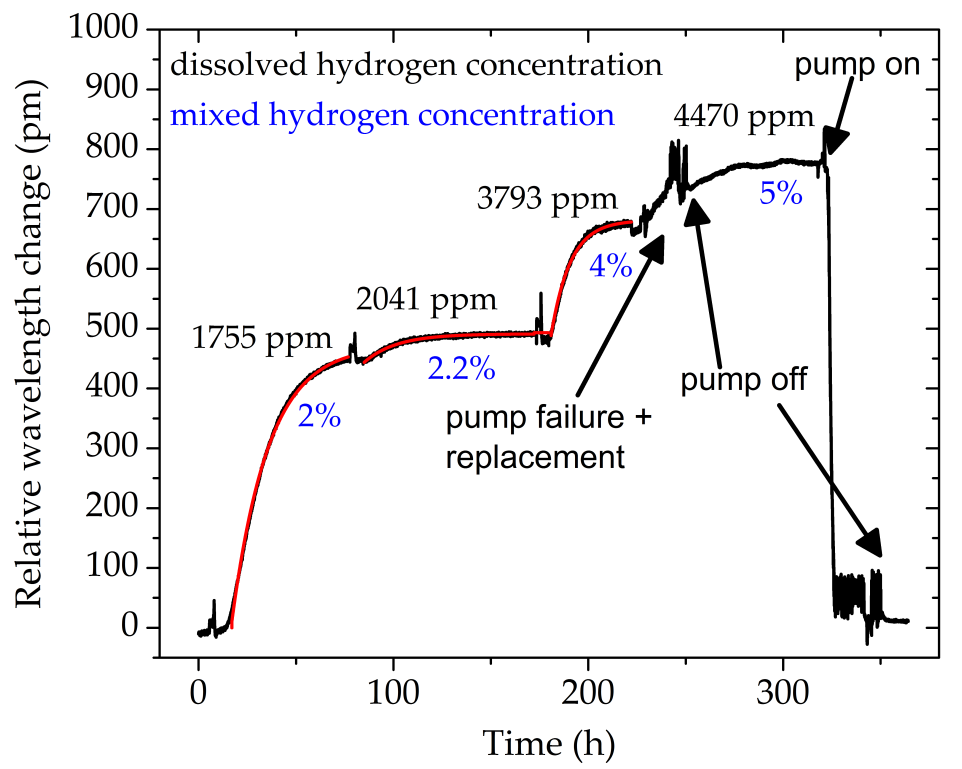

Figure 8.13: Response of a $100 \mu \mathrm{m}$ palladium foil sensor (second segment). The exponential decay fit is also displayed (red). The hydrogen concentrations are given in \% for prepared gas mixture (red) and in ppm for the measured, dissolved hydrogen concentration (blue).

8.12 and Figure 8.13 . The other 14 sensors behaved similarly. The signal is significantly noisier than the measurement in gas (see Figure 8.1). This is caused by stronger fluctuations in temperature $\left(89.5 \pm 1.4^{\circ} \mathrm{C}\right)$ and pressure $(1062 \pm 20$ mbar) due to strong fluctuations in the room temperature $\left(22.5 \pm 3^{\circ} \mathrm{C}\right)$ and the sampling, and backfilling of the oil for the dissolved gas analysis. The sharp peaks in Figure 8.12 and Figure 8.13 are caused by fluctuations in the oil temperature when sampling.

Each step was held for a maximum of seven days. Before the gas mixture in the system was changed, four oil samples were taken. The dissolved hydrogen concentration was determined by averaging over the analysed samples. The first three samples were analysed and if the analysed dissolved hydrogen concentrations were not within $10 \%$ of each other, the $4^{\text {th }}$ sample was analysed as well. The measured dissolved hydrogen concentration is given in Table 8.1 and Table 8.2. For the first segment only one sample was analysed for the $0 \%$ hydrogen steps. For the second segment 4 samples where analysed for the $0 \%$ steps. These measurements showed that there is residual hydrogen in the system, even after full unloading of the sensors. However, the detected values are close to the lower detection limit of 5 ppm of the Myrkos (gas chromatograph system for dissolved gas analysis of transformer oil, see Section 3.4.3). 
Table 8.1: Hydrogen concentration of the bubbled gas mixture (\%) and dissolved gas concentration ( $\mathrm{ppm}$ ) measured using the Myrkos for the first segment.

\begin{tabular}{lrrrrrrrrr}
\hline Gas concentration (\%) & 0 & 0.01 & 0.05 & 0.1 & 0.25 & 0.5 & 0.66 & 1 & 0 \\
\hline DGA1 (ppm) & 0 & 0 & 25 & 65 & 154 & 310 & 361 & 530 & 0 \\
DGA2 (ppm) & & 9 & 30 & 66 & 157 & 306 & 360 & 530 & \\
DGA3 (ppm) & & 10 & 31 & 65 & 158 & 311 & 348 & 520 & \\
DGA4 (ppm) & & 0 & 29 & & & & & & \\
\hline
\end{tabular}

Table 8.2: Hydrogen concentration of the bubbled gas mixture (\%) and dissolved gas concentration ( $\mathrm{ppm}$ ) measured using the Myrkos for the first segment.

\begin{tabular}{lrrrrrr}
\hline Gas concentration (\%) & 0 & 2 & 2.43 & 4 & 5 & 0 \\
\hline DGA1 (ppm) & 6 & 1727 & 2175 & 3453 & 4391 & 8 \\
DGA2 (ppm) & 8 & 1833 & 1940 & 3631 & 4498 & 8 \\
DGA3 (ppm) & 5 & 1705 & 2008 & 3394 & 4521 & 0 \\
DGA4 (ppm) & 5 & & & & & 6 \\
\hline
\end{tabular}

To determine the final wavelength change an exponential decay fit was used, which also describes the hydrogen response in oil well (see Figure 8.12). For the first $0.01 \%$ (5 ppm dissolved) hydrogen step the exponential decay fit did not give satisfactory results, as well as, for the 5\% (4470 ppm dissolved) hydrogen step. This is due to the high signal to noise ratio in both steps, caused by the low signal in the 5 ppm step and the failure of the pump in the $4470 \mathrm{ppm}$ step. Here, the final wavelength change was chosen as measured just before the oil sampling.

The dissolved hydrogen sensitivity of a $100 \mu \mathrm{m}$ palladium foil sensor is shown in Figure 8.14. The values for the dissolved hydrogen concentration are the averaged DGA values. Next to the experimental results the calculated sensitivity is displayed. The sensitivity was calculated using coefficient of strain transfer $k_{\mathrm{ST}}$ determined for the 5\%/ $4470 \mathrm{ppm}$ step. For the displayed sensor $k_{\mathrm{ST}}=1.11$. 


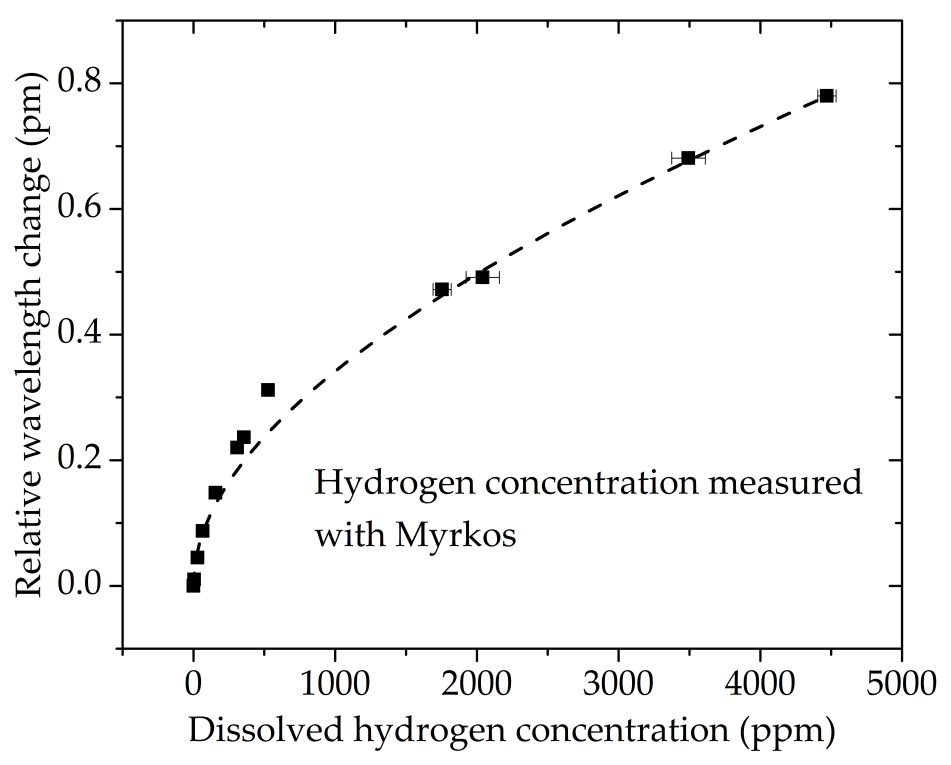

Figure 8.14: Dissolved hydrogen sensitivity of a $100 \mu \mathrm{m}$ palladium foil sensor. The dissolved hydrogen concentration was measured with the Myrkos. Also displayed the predicted sensitivity (dashed lines) of the sensor calculated using $k_{\mathrm{ST}}=1.11$.

\subsubsection{Discussion}

In first sight, the predicted and measured values in Figure 8.14 seem to correlate well. However, a closer look reveals that the measurements at concentrations up to 530 ppm follow a different trend than the measurements at higher concentrations.

The Myrkos has a linear measurement range for dissolved hydrogen from 5-700 ppm. If the analysed sample is outside this range, a dilution procedure is followed and the sample is analysed again.

To show the impact of the dilution on the measurement, we separated the DGA measurements into two parts. The first part is in the linear range of the Myrkos and includes all measurements from the first segment (0-1\% hydrogen gas mixture/0-530 ppm dissolved hydrogen). The second part includes the higher concentration for which a dilution procedure was necessary. Figure 8.15 shows the dissolved hydrogen concentration measurements with the myrkos versus the hydrogen concentration of the gas mixture that was bubbled through the oil. A linear fit was applied to measurements of the low and high concentrations. The slopes of both lines diverge with $0.054 \pm 0.002$ for the lower concentrations and $0.089 \pm 0.002$ for the higher concentrations. The slope of the line represents the Ostwald coefficient $\left(k_{\text {Ost }}=\right.$ concentration in the liq- 


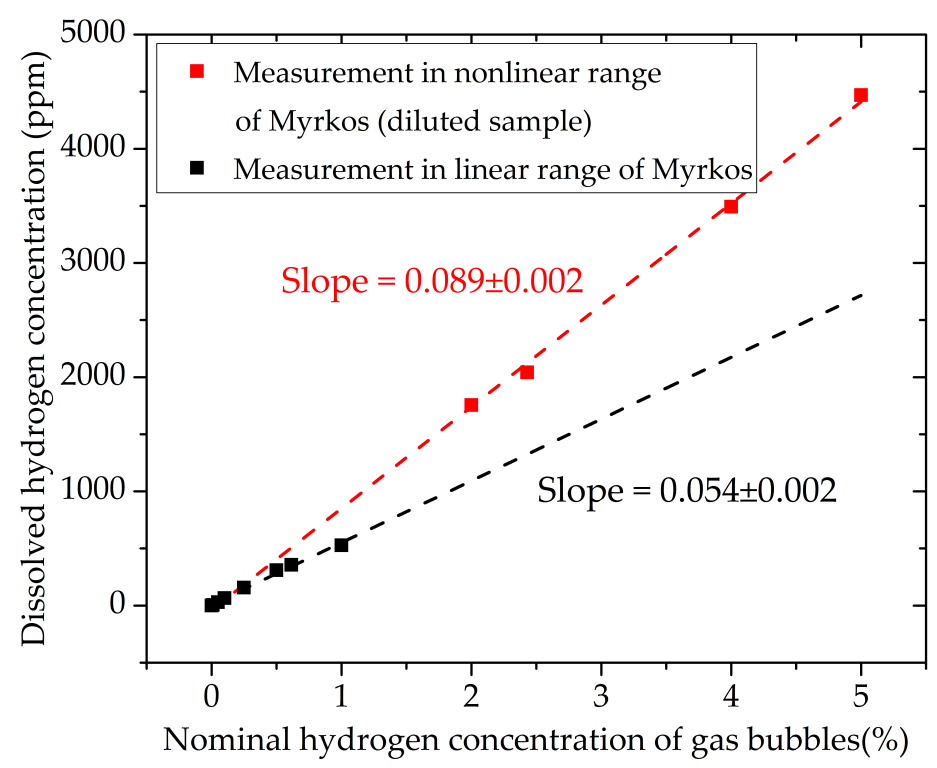

Figure 8.15: Dissolved hydrogen concentration measured with Myrkos versus hydrogen concentration of the bubbled gas.

uid phase/concentration in the gas phase) and should be independent of the hydrogen concentration (see Section 2.1.5). This indicates that there is a systematic error in how the dissolved hydrogen concentration is calculated in the Myrkos for values out of the linear range of the equipment.

Instead of measuring the dissolved gas concentration with the Myrkos, the concentration can be calculated from the hydrogen concentration of the gas mixture and the Ostwald coefficient. The dissolved hydrogen sensitivity of a $100 \mu \mathrm{m}$ palladium foil sensor where the dissolved hydrogen concentration is calculated is shown in Figure 8.16. The experimental results are now in better agreement with the calculated sensitivity. For the calculation $k_{\mathrm{ST}}=1.03$ (determined during the characterisation in gas) was used.

\subsection{Conclusion}

In this chapter fifteen $100 \mu \mathrm{m}$ palladium foil sensor were characterised in gas and oil environments. The 15 sensors were manufactured simultaneously with the vacuum bagging method and showed high reproducibility. This proves the scalability of the manufacturing method and suitability for commercial use.

All sensors showed similar response to hydrogen and had a high accuracy and precision in gas and oil. This proves their suitability for the implementation in a transformer for field testing. 


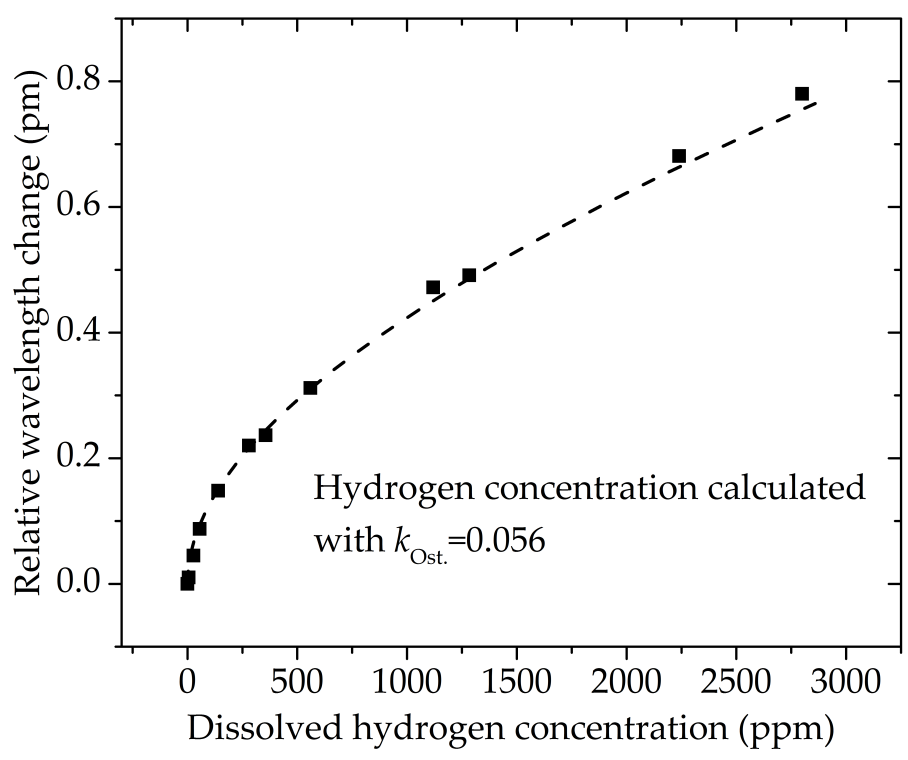

Figure 8.16: Dissolved hydrogen sensitivity of a $100 \mu \mathrm{m}$ palladium foil sensor. Dissolved hydrogen concentration calculated with an Ostwald coefficient of 0.056. Also displayed the predicted sensitivity (dashed lines) of the sensor determined with $k_{\mathrm{ST}}=1.03$.

The effect of a thin film silicon dioxide membrane on the carbon monoxide cross-sensitivity was investigated in gas atmosphere. No significant improvement could be observed. In general, the sensors showed a slowed response in the presence of carbon monoxide, but the sensitivity was not affected. Since response time is not a main optimization parameter for the application in a transformer, no further effort has been dedicated to the development of a membrane to mitigate the influence of carbon monoxide on the hydrogen measurement.

The mobile gas chromatograph Myrkos showed inconsistencies in the results for measurements out of the linear range of the equipment (concentration larger 700 ppm dissolved hydrogen). Therefore, we chose to calculate the dissolved hydrogen concentration via the Ostwald coefficient and the nominal hydrogen concentration of the gas phase for the further analysis in Chapter 9. 


\section{Chapter 9}

\section{Investigation of the coefficient of strain transfer and its influence on the sensor calibration}

As mentioned in Chapter 4, each sensor requires calibration in order to accurately measure hydrogen. We showed that a calibration can be performed by determining a coefficient of strain transfer $k_{\mathrm{ST}}$ (see Equation 4.6 and 4.7)

In the following three subsections, three different methods are discussed for determining the coefficient of strain transfer: (a) via a hydrogen measurement, where the expanding palladium (due to hydrogen absorption) strains a surface bonded FBG, (b) via a temperature measurement, where the thermal expansion of a body (e.g., palladium, steel) strains a surface bonded FBG, (c) via a strain measurement, where the mechanical expansion of a body (e.g., palladium, steel) strains a surface bonded FBG.

\subsection{Determining the coefficient of strain transfer via a hydrogen measurement}

The coefficient of strain transfer can be determined via a single-point hydrogen measurement. For optical fiber sensors, this method has not been reported in the literature before. However, Peng et al. used a correction factor to fit their theoretical prediction to their measurements [109]. Their correction factor was around 1.3 and was correlated to uncertainties in their equations. It was not correlated to a coefficient of strain transfer and not determined via a single-point hydrogen measurement. The coefficient of strain transfer can be determined as follows. At a constant temperature, the second term of (4.7) is 


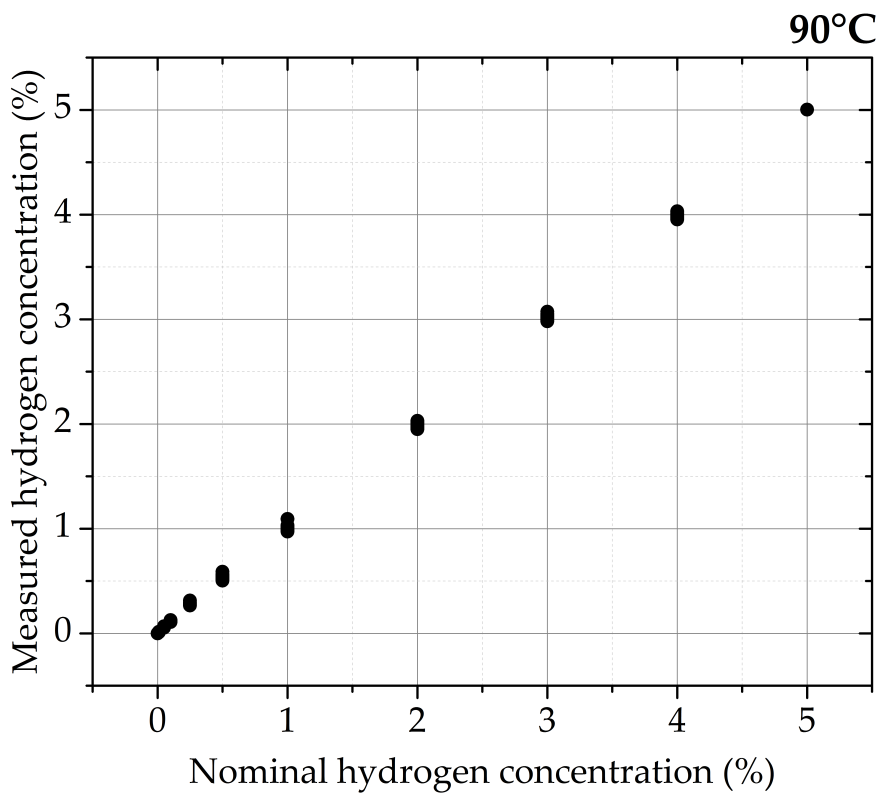

Figure 9.1: Measured (using equation (4.8)) versus the nominal (mixed from calibrated cylinders) hydrogen concentration. Displayed are the results of all 15 sensors. The $k_{\mathrm{ST}}$ value used was determined at $90^{\circ}$ and $5 \%$ hydrogen.

zero and $k_{\mathrm{ST}}$ for the hydrogen measurement can be calculated as

$$
k_{\mathrm{ST}}=\frac{\Delta \lambda}{k_{\varepsilon} \Delta \varepsilon^{\prime}}=\frac{\Delta \lambda_{\mathrm{H} 2 \text { measured }}}{\Delta \lambda_{\mathrm{H} 2 \text { _calculated }}}
$$

which is the ratio of experimental to calculated wavelength change of the FBG due to the strain induced by the expanding palladium hydride. Furthermore, the coefficient of strain transfer determined via this method can be used as a general calibration constant as it absorbs uncertainties in the variables of $k_{\varepsilon}$, $C$, and the geometrical and material properties $A_{\mathrm{Pd}}, A_{\mathrm{f}}, E_{\mathrm{Pd}}, E_{\mathrm{f}}$ (see equation (4.10)).

\subsubsection{Influence on the sensor calibration}

The coefficient of strain transfer was determined for the fifteen $100 \mu \mathrm{m}$ palladium foil sensors discussed in Chapter 8. Using equation (4.8) the hydrogen concentration in gas was calculated. The measured (using (4.8)) versus the nominal (mixed from calibrated cylinders) hydrogen concentration at $90^{\circ} \mathrm{C}$ of all 15 sensors is displayed in Figure 9.1. The linearity and slope $(\approx 1)$ imply that the coefficient of strain transfer (determined via a single point hydrogen measurement) can be used for calibration.

A detailed view of the hydrogen measurement at lower concentrations is displayed in Figure 9.2. The close up shows that the measured hydrogen con- 


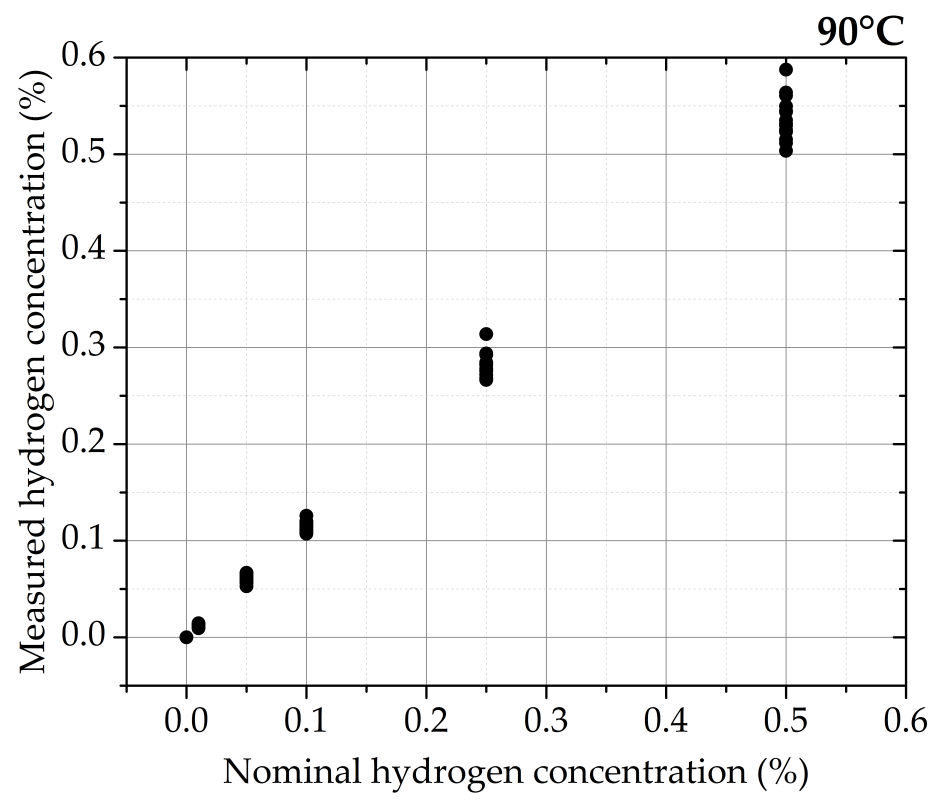

Figure 9.2: Detail view of measured (using equation (4.8)) versus the nominal (mixed from calibrated cylinders) hydrogen concentration. Displayed are the results of all 15 sensors. The $k_{\mathrm{ST}}$ value used was determined at $90^{\circ}$ and $5 \%$ hydrogen.

centration diverges more strongly at low concentrations compared to higher concentrations. This indicates a lower relative accuracy at lower concentrations.

In Figure 9.3 the relative accuracy $\left(=100^{*}\right.$ (measured hydrogen concentration - nominal hydrogen concentration)/nominal hydrogen concentration) of the 15 sensors at the different hydrogen concentrations is shown. The relative accuracy improves for increasing hydrogen concentrations. This is mainly due to the low signal to noise ratio at low concentrations. However, the average errors of the sensors for concentrations below 1\% hydrogen are all positive and higher than $5 \%$ (up to $20 \%$ ). This suggests that there is potential for improvement of the model at low concentrations.

The coefficient of strain transfer determined in gas atmosphere was also used to calibrate the 15 sensors for dissolved hydrogen measurements, after they had been transferred into oil. Using equation (4.9) and an Ostwald coefficient of 0.056 the dissolved hydrogen concentration was calculated. The results are shown in Figure 9.4.

In Figure 9.5 the relative accuracy of the 15 sensors at different dissolved hydrogen concentrations is shown. For the concentrations below 50 ppm the relative accuracy is high and below 0 . This might be due to the sensors (pal- 


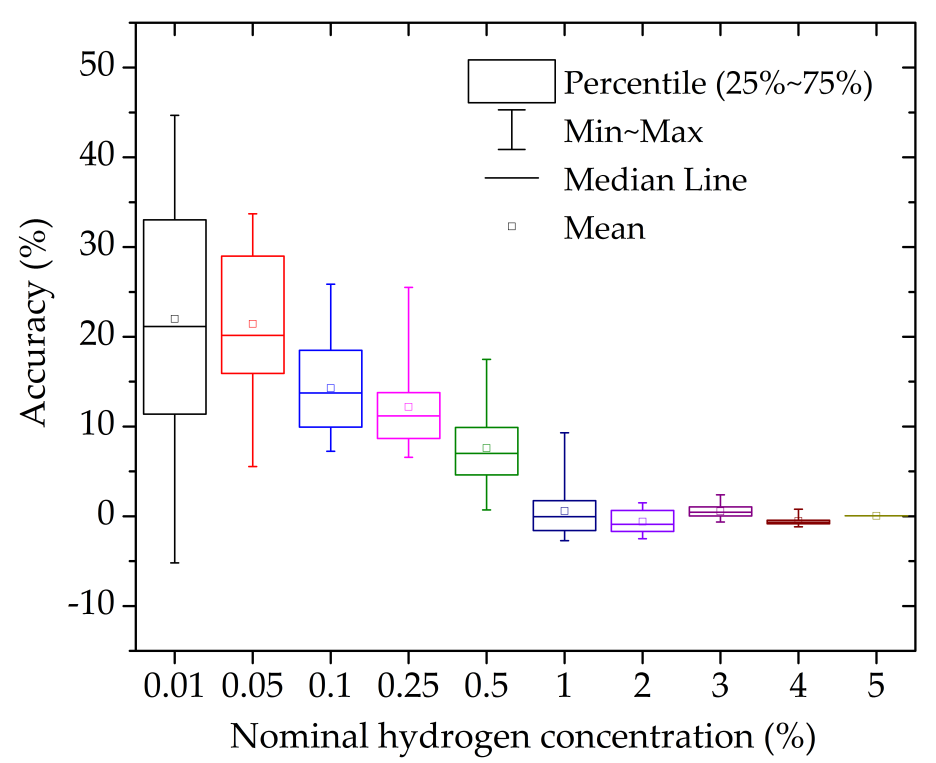

Figure 9.3: Relative accuracy of the 15 sensors (calibrated with $k_{\mathrm{ST}}$ from a hydrogen measurement) at the different hydrogen concentrations. At $5 \%$ the relative accuracy is close to 0 since the calibration was performed for the $5 \%$ step.

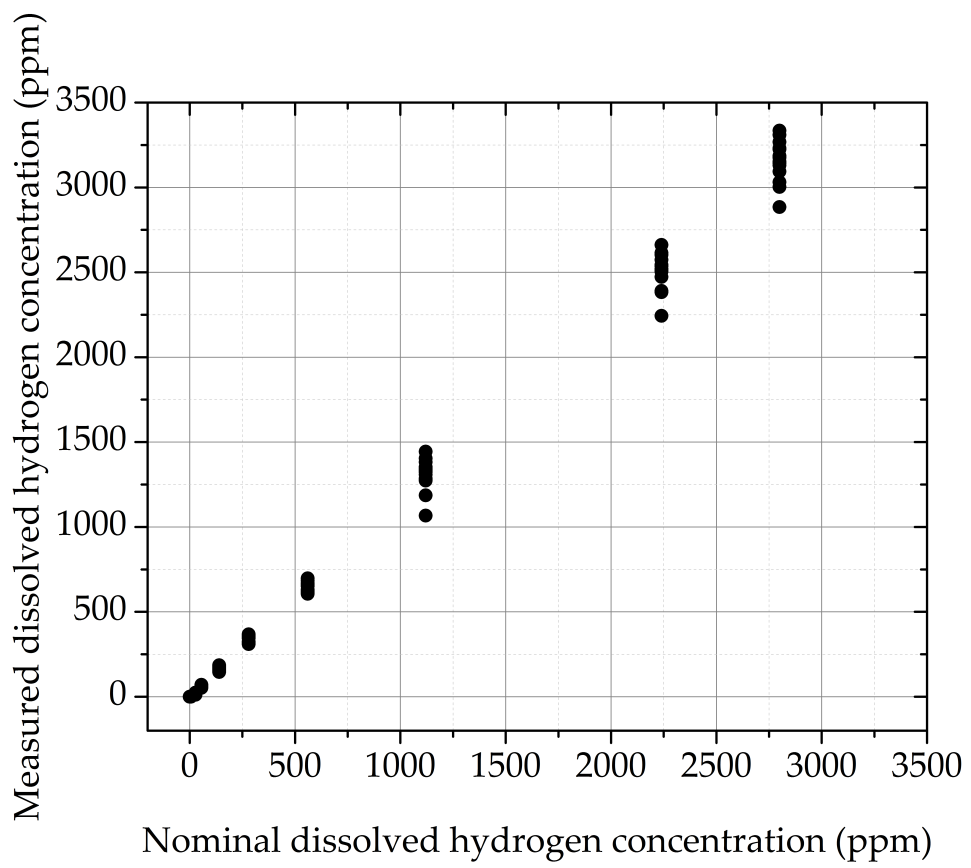

Figure 9.4: Measured (using equation (4.9) and an Ostwald coefficient of 0.056) versus the nominal (determined via bubbled gas concentration) dissolved hydrogen concentration. Displayed are the results of all 15 sensors. The $k_{\mathrm{ST}}$ value used was determined in gas environment at $90^{\circ}$ and $5 \%$ hydrogen. 


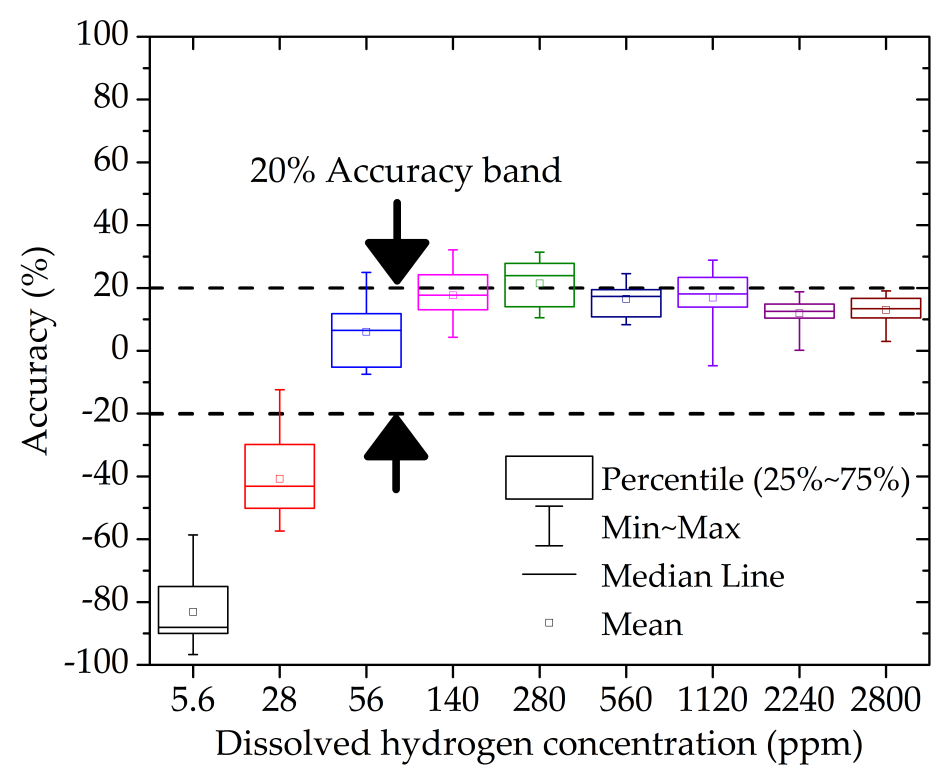

Figure 9.5: Relative accuracy of the 15 sensors (calibrated with $k_{\mathrm{ST}}$ from a hydrogen measurement in gas) at the different dissolved hydrogen concentrations.

ladium) not having equalised with the environment and fully responded after 7 days. For concentrations above 50 ppm the accuracy improves and is close to a $20 \%$ accuracy. However, the mean accuracy of each sensor is above $10 \%$ and correlates to an increased sensitivity of the sensor during the oil measurements. This increase in sensitivity may be due to a temperature lower than $90^{\circ} \mathrm{C}$ at the sensors during the measurements in oil, causing a higher solubility of hydrogen in palladium, and thus expansion. The difference in sensitivity is equivalent to a decrease of around $5^{\circ} \mathrm{C}$ for the oil measurement. This temperature difference in gas and oil measurements might be due to the location of the thermocouple and the different heat transferring medium: For both measurements the same thermocouple was used and located close (around $20 \mathrm{~mm}$ above) to the sensors, but the thermal conduction and temperature gradient are different in gas and oil, which might explain the increase in sensitivity due to inaccurately measured temperature.

Besides determining the coefficient of strain transfer in gas, the coefficient can be determined in oil. For the 2800 ppm dissolved hydrogen measurement the average coefficient increases from 1.03 (in gas) to 1.11 (in oil). The results (using this coefficient and equation (4.9)) are shown in Figure 9.6. The relative accuracy improves for measurements above $50 \mathrm{ppm}$ and is shown in Figure 9.7. 


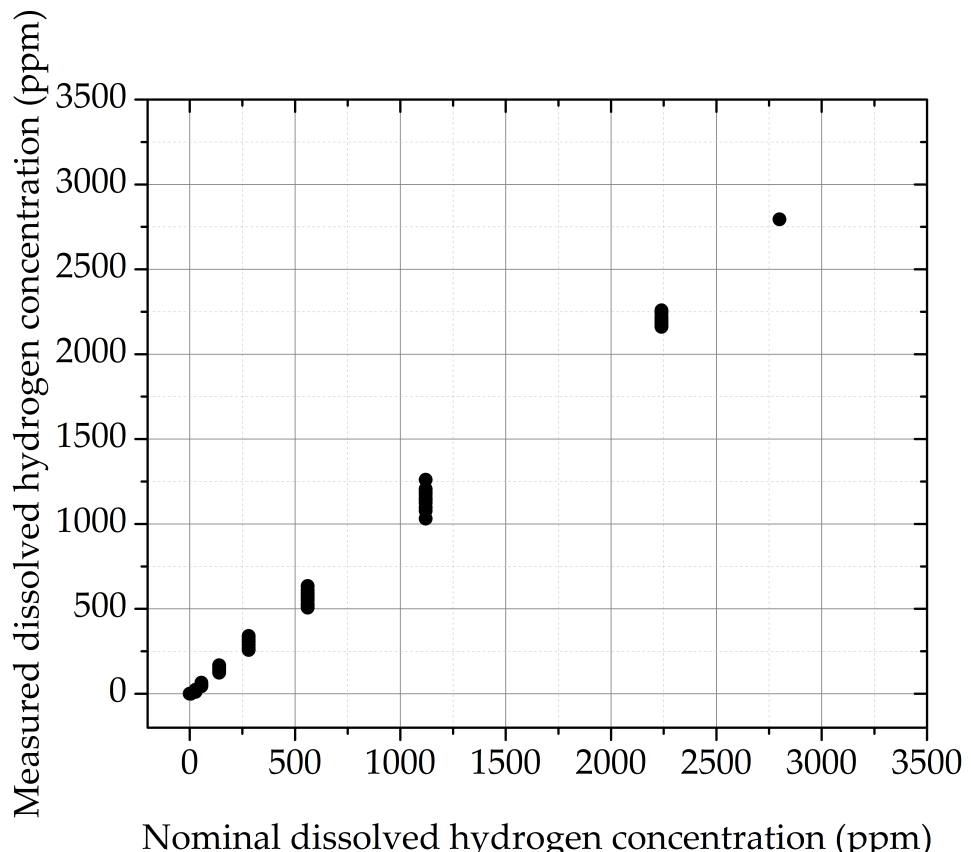

Figure 9.6: Measured (using equation (4.8)) versus the nominal (determined via bubbled gas concentration) dissolved hydrogen concentration. Displayed are the results of all 15 sensors. The $k_{\mathrm{ST}}$ value used was determined in oil environment at $90^{\circ}$ and 2800 ppm dissolved hydrogen.

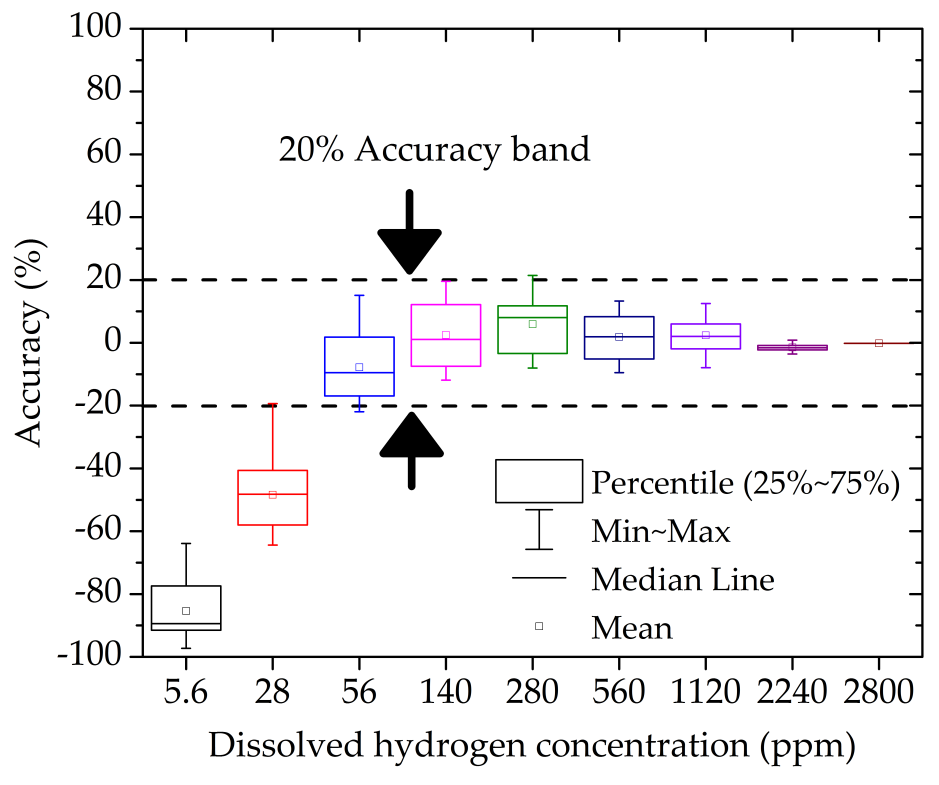

Figure 9.7: Relative accuracy of the 15 sensors (calibrated with $k_{\mathrm{ST}}$ from a hydrogen measurement in gas) at the different dissolved hydrogen concentrations. At 2800 ppm the relative accuracy is close to 0 since the calibration was performed for the 2800 ppm dissolved hydrogen step. 
In general, the coefficient of stain transfer seems to be good parameter for the calibration of the sensors. The advantage of deriving the coefficient from a single point hydrogen measurement is that it requires less measurements than a multi-point calibration. Furthermore, the calibration is derived directly from a hydrogen measurement. The disadvantage is that a test setup which is stable in temperature, pressure, and hydrogen concentration is necessary. Depending on the sensor the hydrogen loading can take several hours which makes this calibration method relatively time intensive.

A hydrogen calibration of the sensors in gas can be used to also calibrate the sensors for measurements in oil. However, the accuracy for dissolved hydrogen measurements increases when the sensors were calibrated in oil instead of gas.

\subsection{Determining the coefficient of strain transfer via a temperature measurement}

With this novel method the coefficient of strain transfer can be determined via a multi-point temperature calibration. To my best knowledge, this method has not been reported in the literature before. By attaching palladium to the FBG, its temperature sensitivity is changed. The sensor is not only sensitive to temperature by the second term $\left(k_{T} \Delta T\right)$ of equation (4.7) but also by the first term $\left(k_{\varepsilon} k_{\mathrm{ST}} \Delta \varepsilon^{\prime}\right)$ due to the thermal expansion of palladium straining the FBG. The expansion $\varepsilon_{\text {Pd_thermal }}$ of palladium due to temperature increase can be calculated as

$$
\varepsilon_{\text {Pd_thermal }}=\frac{\Delta l}{l}=\alpha_{\mathrm{Pd}} \Delta T
$$

where $\alpha_{\mathrm{Pd}}$ is the coefficient of thermal expansion of palladium $\left(\alpha_{\mathrm{Pd}}=11.8\right.$. $10^{-6} /{ }^{\circ} \mathrm{C}$ at $20^{\circ} \mathrm{C}$ [134]). Similar to the expansion of palladium due to hydrogen absorption, the strain of the FBG due to the thermal expansion $\left(\Delta \varepsilon^{\prime}\right)$ of palladium can be calculated using (4.3) by substituting $\varepsilon_{\mathrm{Pd} \_\mathrm{H} 2}$ with $\varepsilon_{\text {Pd_thermal }}$ (assuming perfect strain transfer). However, the temperature response of the FBG with attached palladium is also affected by the strain transfer between palladium and fiber. Therefore, $k_{\mathrm{ST}}$ also needs to be applied in a temperature measurement. Adapting (4.3) and (4.6) for temperature measurement and including these with (9.2) into (4.7) leads to

$$
\Delta \lambda=\left(k_{\varepsilon} k_{\mathrm{ST}} \alpha_{\mathrm{Pd}} \frac{Y}{1+Y}+k_{T}\right) \Delta T=\left(k_{\mathrm{ST}} k_{T \text { Pd }}+k_{T}\right) \Delta T=k_{T \text { Sensor }} \Delta T
$$




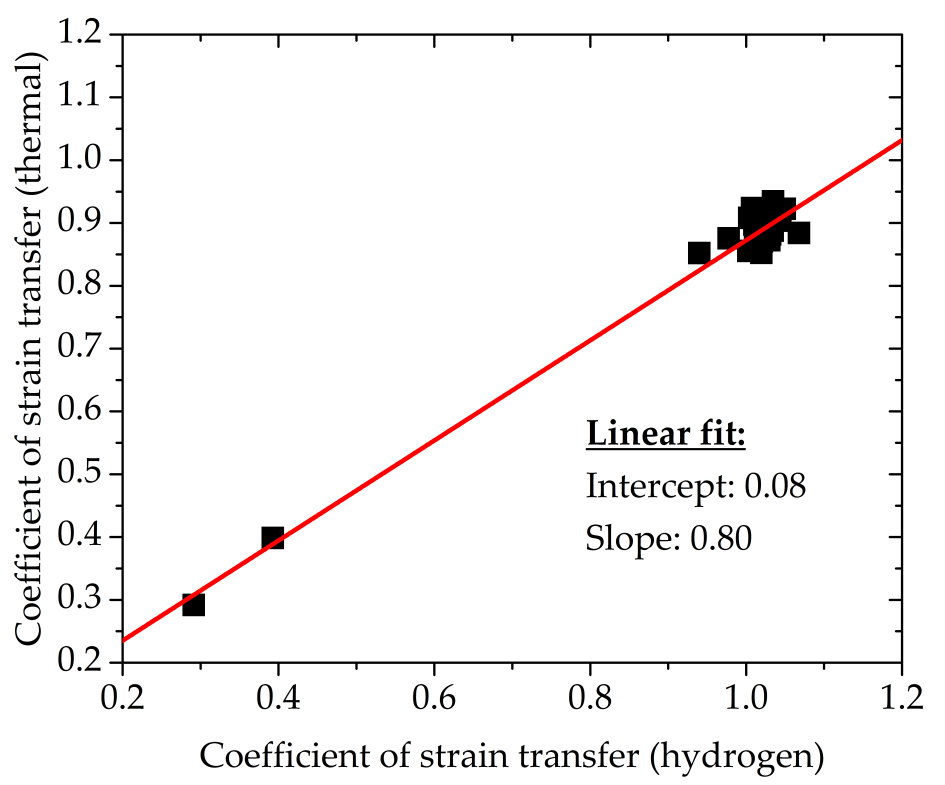

Figure 9.8: Coefficients of strain transfer derived via a temperature (thermal) measurement versus via a hydrogen measurement of all $100 \mu \mathrm{m}$ palladium foil sensors reported in in this thesis. Also displayed is a linear fit.

The increase in temperature sensitivity of the sensor due to the thermal expansion of palladium can thus be expressed using $k_{T} \mathrm{Pd}$. The temperature sensitivity of the whole sensor can be expressed using $k_{\mathrm{ST}}, k_{T}$ Pd, and $k_{T}$, or

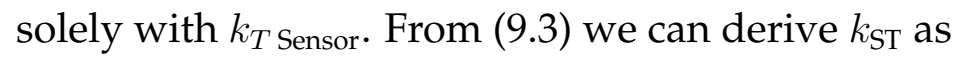

$$
k_{\mathrm{ST}}=\frac{k_{T \text { Sensor }}-k_{T}}{k_{T \text { Pd }}}
$$

where, $k_{T}$ Sensor and $k_{T}$ are meausred using a palladium sensor and an uncoated reference FBG, and $k_{T \text { Pd }}$ is calculated using (9.3). It is also possible to use the literature value $k_{T}=10.4 \mathrm{pm} /{ }^{\circ} \mathrm{C}$ [64].

To compare the coefficients of strain transfer determined via a temperature measurement versus hydrogen measurement, both coefficients were calculated for each of the $100 \mu \mathrm{m}$ palladium foil sensors reported in Chapter 5, 6 and 8. The results are shown in Figure 9.8. Despite the lack of data points over the whole range, a linear fit was applied. A Pearson correlation coefficient of 0.99 indicates correlation of the two coefficient and the fit has a slope of 0.80 with an intercept of 0.08 .

Ideally the slope of the fit would be 1 with an intercept of 0 to show that the coefficient estimate is independent of the method of determination. The deviation can be explained as follows. Both methods rely on several literature values for the calculations of the coefficients. Particularly, the constants $C$ 
and $\alpha_{\mathrm{Pd}}$ are likely candidates influencing this correlation. The impact of both can be equalized by multiplication with a correction factor. We determined the correction factor by the ratio of the average value of coefficient of strain transfer via a hydrogen measurement versus temperature measurement. The ratio/correction factor is 1.12 for the coefficients shown in Figure 9.8.

The coefficient of strain transfer was not deliberately altered, but varied due to the manufacturing methods. Therefore, only a small amount of different values for the coefficients could be analysed. However, the strain transfer coefficient can be deliberately altered by varying the the coating material of the fiber, the bond length and bond line thickness. Varying these parameters is difficult with the chosen sensor designs. The fiber (and coating) diameter is determined by the groove, which was designed for an uncoated, bare fiber, the bond length is determined by the palladium foil length, and the bond line thickness was reduced to a minimum with the vacuum bagging method. Therefore, another experiment was designed in which the coefficient of strain transfer determined via a temperature measurement is compared to the coefficient of strain transfer via a (mechanical) strain measurement. Here, the mechanical strain induced by a tensile test machine is replacing the strain induced by the expanding palladium (this experiment is described in Section 9.3).

\subsubsection{Influence on the sensor calibration}

This calibration method is new and original. It is based on the assumption that the coefficient of strain transfer determined via a hydrogen measurement is the same as determined via a temperature measurement. This assumption is derived from the thought that the volumetric expansion of palladium due to hydrogen absorption and the thermal expansion of palladium due to temperature changes are subject to the same constraints when measured with an FBG.

The coefficient of strain transfer was calculated for fifteen $100 \mu \mathrm{m}$ palladium foil sensors discussed in Chapter 8. Using equation (4.8) and a correction factor of 1.12 the hydrogen concentration was calculated. The measured hydrogen concentration at $90^{\circ} \mathrm{C}$ of all 15 sensors is displayed in Figure 9.9. The linearity and slope $(\approx 1)$ imply that the coefficient of strain transfer (determined via a temperature measurement) can be used for calibration. However the calibrated sensors diverge more strongly in terms of measured hydrogen concentration (compare Figure 9.1 and 9.9).

In Figure 9.10 the relative accuracy of the fifteen sensors at the different hy- 


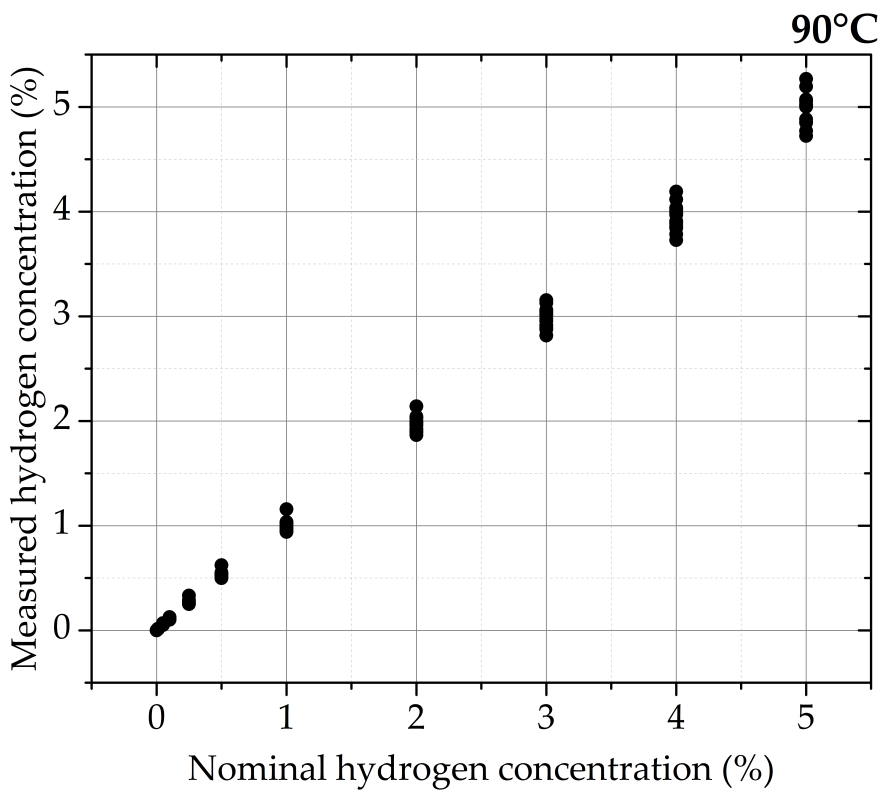

Figure 9.9: Measured (using equation (4.8)) versus the nominal (mixed from calibrated cylinders) hydrogen concentration. $k_{\mathrm{ST}}$ was determined via a temperature measurement.

drogen concentrations is shown. In a manner similar to the calibration with hydrogen measurements, relative accuracy improves for increasing hydrogen concentrations. Again, this is mainly due to the low signal to noise ratio at low concentrations. Also, the average errors of the sensors for concentrations below $1 \%$ hydrogen are again all positive and higher than $5 \%$ (up to $20 \%$ ). This indicates that there is potential for improvement of the model at low concentrations.

The coefficient of strain transfer determined via temperature measurement was also used to calibrate the 15 sensors for dissolved hydrogen measurements in oil. The results are shown in Figure 9.11. In Figure 9.12 the relative accuracy of the 15 sensors at different dissolved hydrogen concentrations is shown.

Comparing the achieved accuracy shows that the relative accuracy of the calibrated sensors depends on how the coefficient of strain transfer is determined: via a temperature measurement shows decreased accuracy especially at concentrations above $1 \%$ hydrogen compared to via a hydrogen measurement for measurements in gas environment (compare Figure 9.3 and Figure 9.10). But, for measurements in oil both methods give similar accuracy results (compare Figure 9.5 and Figure 9.12).

Determining the coefficient of strain transfer via a temperature measurement and using this method for hydrogen calibration has the advantages of 


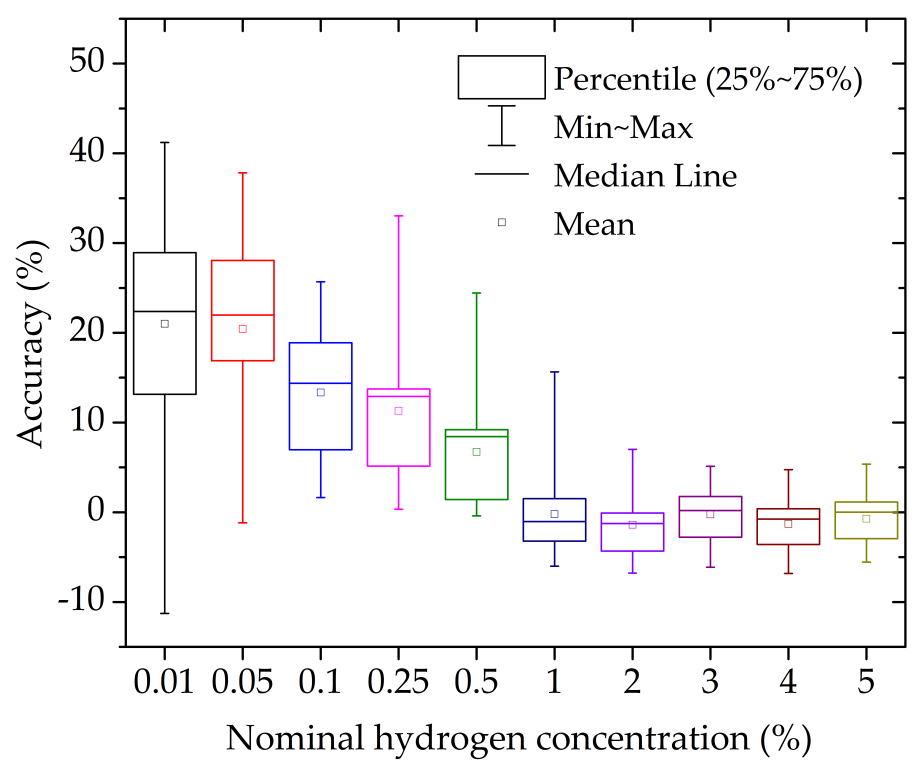

Figure 9.10: Relative accuracy of the sensors (calibrated with $k_{\mathrm{ST}}$ from temperature measurement) at the different hydrogen concentrations.

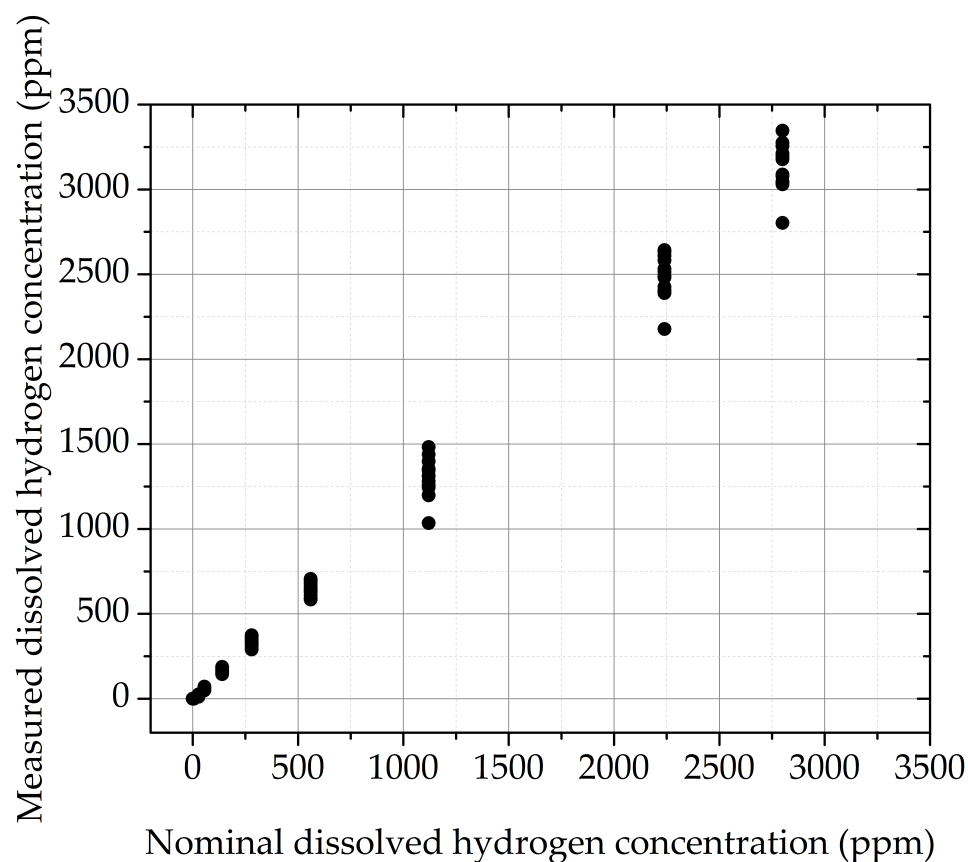

Figure 9.11: Measured (using equation (4.9) and an Ostwald coefficient of 0.056) versus the nominal (determined via bubbled gas concentration) dissolved hydrogen concentration. Displayed are the results of all 15 sensors. $k_{\mathrm{ST}}$ was determined via a temperature measurement. 


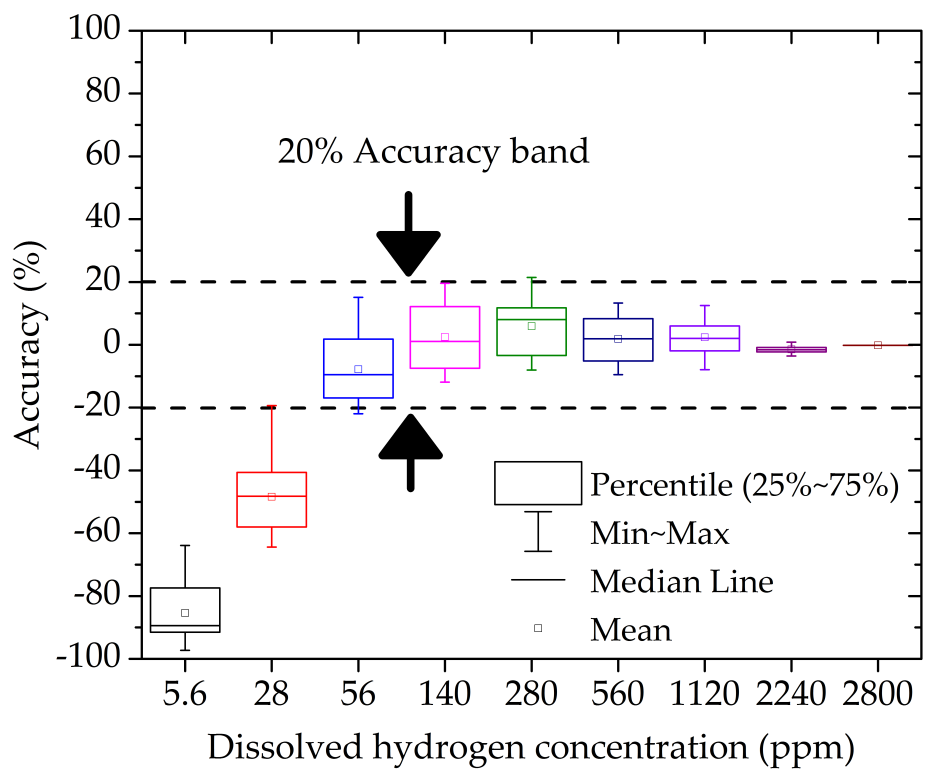

Figure 9.12: Relative accuracy of the 15 sensors (calibrated with $k_{\mathrm{ST}}$ from a temperature measurement in gas) at the different dissolved hydrogen concentrations.

reduced time and cost: (a) The hydrogen response of a sensor can take several hours, or days, whereas a temperature calibration can be performed faster; (b) No special gas calibration facility is necessary, a simple temperature test setup would be sufficient.

\subsection{Determining the coefficient of strain transfer via a strain measurement}

Finally, the coefficient of strain transfer can be determined via a strain measurement as follows. In this section the palladium is substituted with a mild steel structure. This is done because large enough palladium bodies to attach a resistive foil strain gauge and load it in a tensile tester are rather expensive.

If a structure is strained by $\varepsilon_{\text {Structure, }}$ only a certain fraction is transmitted to the surface bonded FBG due to imperfect adhesion and the elasticity of the strain transferring medium (e.g. epoxy adhesive). The tensile test bar has a large cross sectional area (CSA) compared to the fiber which simplifies equation (4.3) to $\varepsilon^{\prime}=\varepsilon_{\text {Structure }}$ and leads to [140]

$$
\Delta \varepsilon=k_{\mathrm{ST}} \varepsilon_{\text {Structure }}
$$




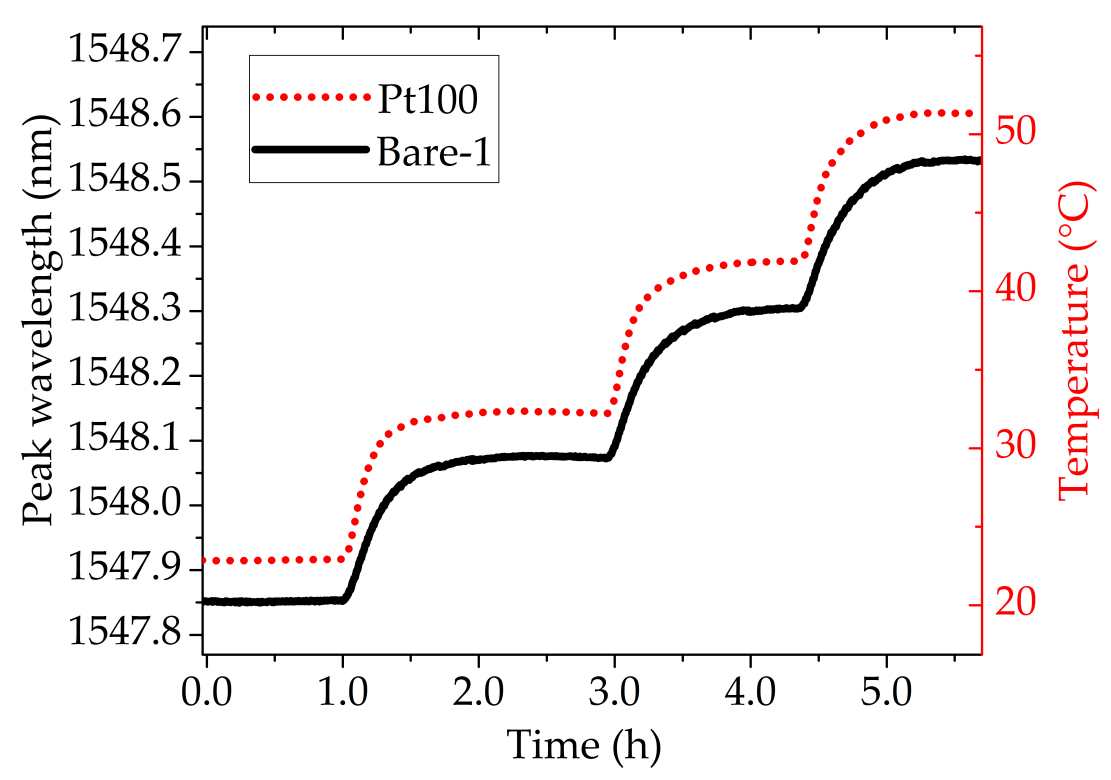

Figure 9.13: Temperature measurement. Displayed are the signals of a Pt100 and a bare FBG.

If the structure is exposed to mechanical strain the coefficient of strain transfer can be expressed as

$$
k_{\mathrm{ST}}=\frac{\Delta \varepsilon}{\varepsilon_{\text {Structure }}}=\frac{\Delta \lambda}{k_{\varepsilon} \varepsilon_{\text {Structure }}}
$$

In the following, an experiment is described in which the coefficient of strain transfer is determined via a strain measurement and via a temperature measurement. The coefficient of strain transfer is deliberately altered by varying the bond length and coating material of the fiber (see Section 3.5). This enables an assessment of the correlation between the two coefficients.

\subsubsection{Experimental method}

The test bars were placed in a temperature controlled chamber to obtain temperature calibration measurements. The temperature was increased in $\sim 10^{\circ} \mathrm{C}$ steps between $20^{\circ} \mathrm{C}$ and $50^{\circ} \mathrm{C}$ (see Figure 9.13). Each step was held for several minutes to allow FBG response to stabilize.

Uni-axial tensile tests were performed between 1 and $3 \mathrm{kN}$. The test bars were carefully aligned in the center line of the axis, and pre strained with 1 $\mathrm{kN}$. Then, with a set speed of $0.3 \mathrm{~mm} / \mathrm{min}$, the tensile force was increased in $0.5 \mathrm{kN}$ steps. Each step was held for $120 \mathrm{sec}$. Once the tension force reached $3 \mathrm{kN}$ the force was decreased to $1 \mathrm{kN}$ in the same manner. This cycle was repeated three times (see Figure 9.14). 


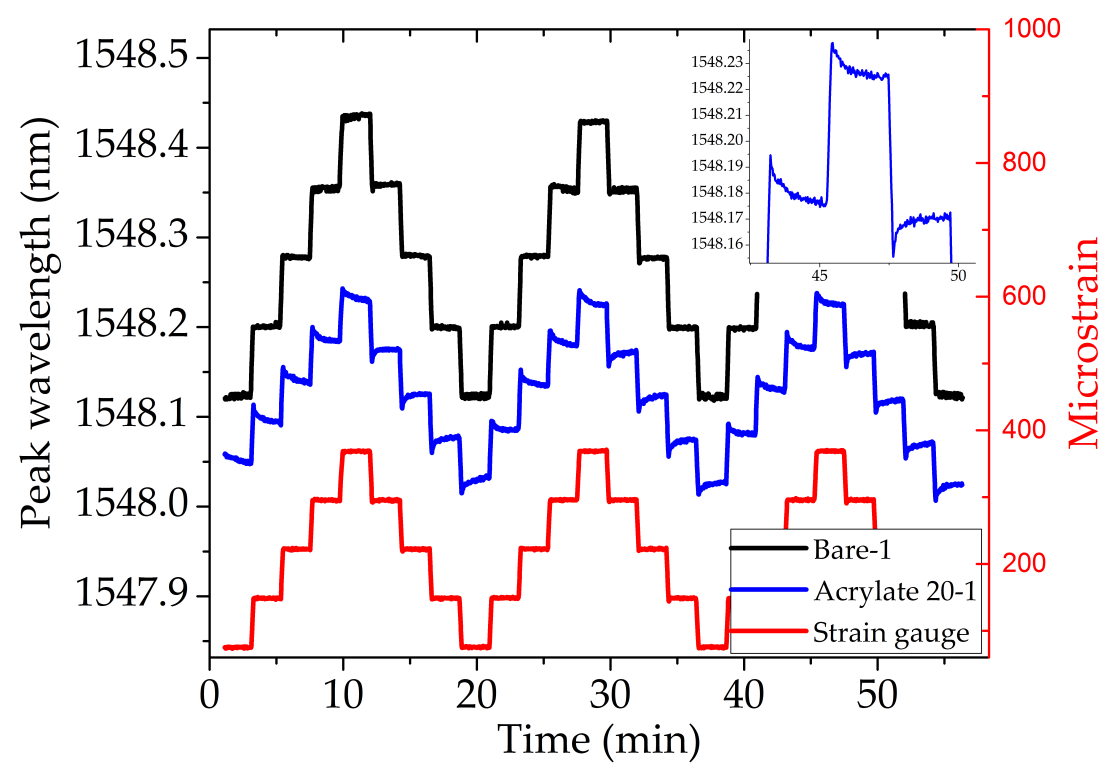

Figure 9.14: Strain measurement. Displayed are the signals of a foil strain gauge, a bare FBG, and an FBG with a acrylate re-coating and $20 \mathrm{~mm}$ bond length.

\subsubsection{Results}

The temperature sensitivity of three surface bonded FBGs (Bare, acrylate with $20 \mathrm{~mm}$, and $60 \mathrm{~mm}$ bond length) is displayed in Figure 9.15. Also displayed is the literature value of a strain isolated (freestanding, not attached to a bar), bare (uncoated) FBG with $\mathrm{k}_{T}=10.4 \mathrm{pm} /{ }^{\circ} \mathrm{C}$ [64]. The increase in in temperature sensitivity (slope of the lines) of the surface bonded FBGs, compared to a strain isolated FBG, is due to the strain which is transferred from the thermal expansion of the test bar. The temperature sensitivity of all FBGs is given in Table 9.1. The surface bonded Ormocer T coated FBGs have the highest temperature sensitivity, followed by the bare FBGs, and the acrylate coated FBGs. The temperature sensitivity increases with bond length for the acrylate coated FBGs.

Figure 9.14 shows the strain response of a surface-bonded bare FBG, an acrylate coated FBG with $20 \mathrm{~mm}$ bond length, and the biaxial resistive strain gauge. The inset in Figure 9.14 highlights a hysteresis phenomenon which could be observed in all acrylate recoated fibers. The hysteresis is assumed to be due to a slipping of the fiber inside the acrylate re-coating. Therefore, the strain sensitivity of all FBGs was determined once the response had stabilized, which occurred during the last 60 seconds of each constant force hold.

The strain sensitivity of a surface bonded bare FBG, an acrylate coated FBG 


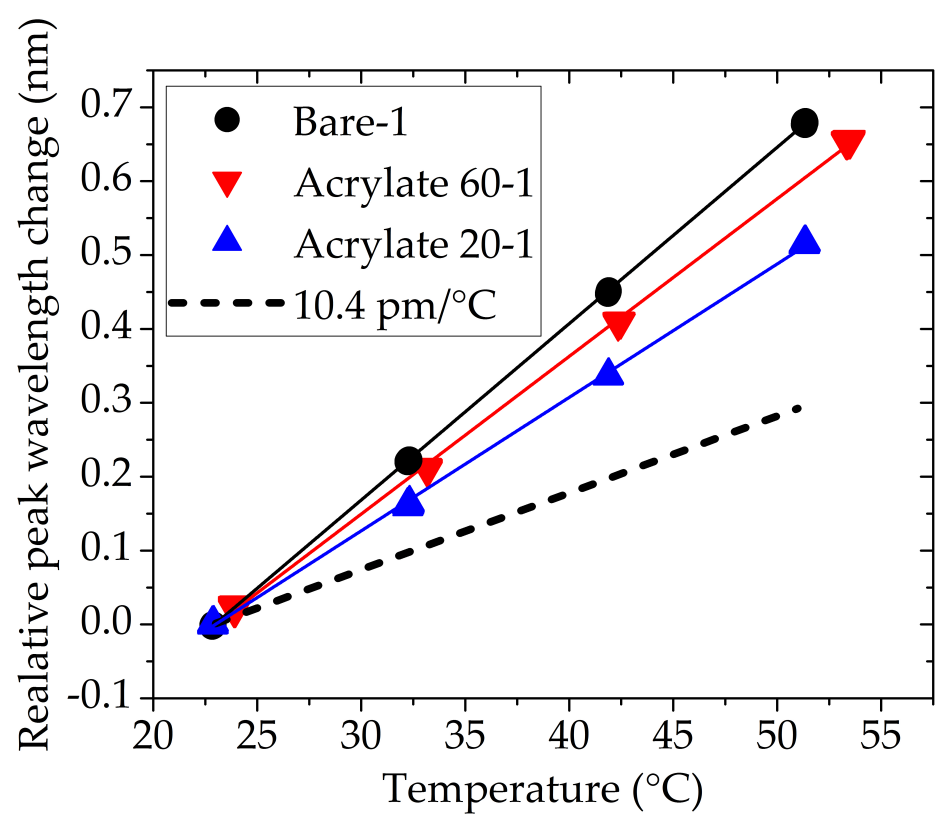

Figure 9.15: Temperature sensitivity of selected FBGs. Literature value of temperature sensitivity of FBG (dashed).

Table 9.1: Temperature sensitivity (T) in $\mathrm{pm} /{ }^{\circ} \mathrm{C}$ and strain sensitivity (S) in $\mathrm{pm} / \mu \varepsilon$ of the Acrylate (A), Bare (B), and Ormocer T $(\mathbf{O})$ coated fiber. Nomenclature:A20-1=Acrylate coating,Bond length 20 mm,FBG 1.

\begin{tabular}{rrrrrrrrrr}
\hline & A20-1 & A20-2 & A20-3 & A40-1 & A40-2 & A40-3 & A60-1 & A60-2 & A60-3 \\
\hline T & 18.1 & 18.4 & 17.9 & 20.1 & 20 & 19.5 & 21.3 & 20.3 & 21.7 \\
$\mathbf{S}$ & 0.65 & 0.68 & 0.58 & 0.71 & 0.8 & 0.71 & 0.94 & 0.85 & 0.96 \\
\hline & $\mathbf{A 8 0 - 1}$ & $\mathbf{A 8 0 - 2}$ & $\mathbf{A 8 0 - 3}$ & $\mathbf{B 2 0 - 1}$ & $\mathbf{B 2 0 - 2}$ & $\mathbf{B 2 0 - 3}$ & $\mathbf{O 2 0 - 1}$ & O20-2 & O20-3 \\
\hline $\mathbf{T}$ & 21.6 & 21.9 & 22.2 & 23.9 & 24 & 24.2 & 24.3 & 24 & 24.1 \\
$\mathbf{S}$ & 0.95 & 1.01 & 0.99 & 1.06 & 1.05 & 1.07 & 1.16 & 1.18 & 1.17 \\
\hline
\end{tabular}




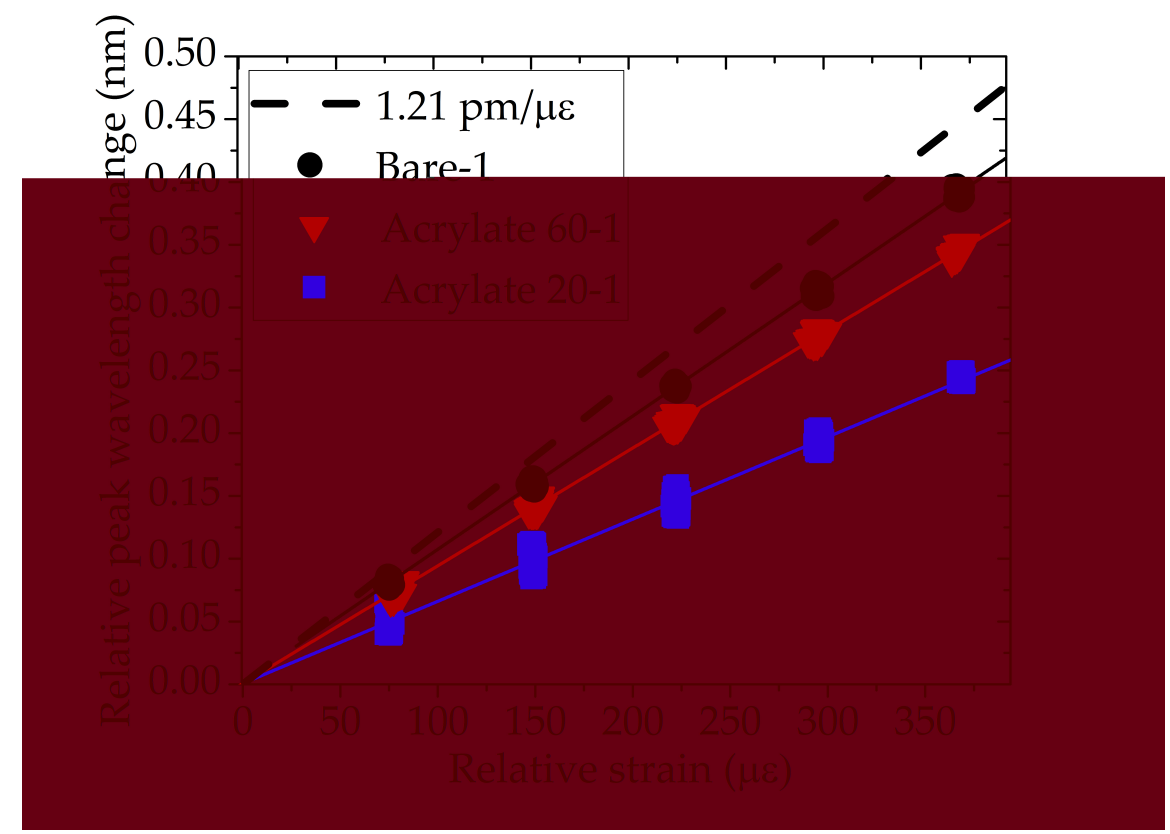

Figure 9.16: Temperature sensitivity of selected FBGs. Literature value of temperature sensitivity of FBG (dashed).

with $20 \mathrm{~mm}$, and $60 \mathrm{~mm}$ bond length is shown in Figure 9.16. Also displayed is the theoretical, maximum strain sensitivity $k_{\varepsilon}=1.21 \mathrm{pm} / \mu \varepsilon$ [64]. The deviation of the strain sensitivity of the surface bonded FBGs from the maximum value is due to the coefficient of strain transfer being $<1$ in all cases. The strain sensitivity of all FBGs is given in Table 9.1. The Ormocer T coated FBGs have the highest strain sensitivity, followed by the bare FBGs, and the acrylate coated FBGs. The strain sensitivity increases with bond length for the acrylate coated FBGs.

Using equations (9.4) and (9.6) the coefficient of strain transfer of the mechanical, and thermal measurement can be calculated. The results of the calculation for the 18 FBGs are shown in Figure 9.17. Applying a linear fit gives a slope of 1.01 and an intercept of -0.02 .

The results show that there is a strong, linear correlation between the coefficient of strain transfer obtained by mechanical tensile test and thermal temperature calibration. A slope close to 1 and an intercept close to 0 suggests, that the coefficients can be considered functionally equivalent. This implies that a surface bonded FBG can be strain calibrated via a temperature measurement. The temperature calibration could be done via daily, intra-day temperature fluctuations or local heating of the structure. With a strain calibrated FBG, absolute displacement and strain measurements are possible, and the health of an individual sensor may be tracked through periodic evaluation of the thermal 


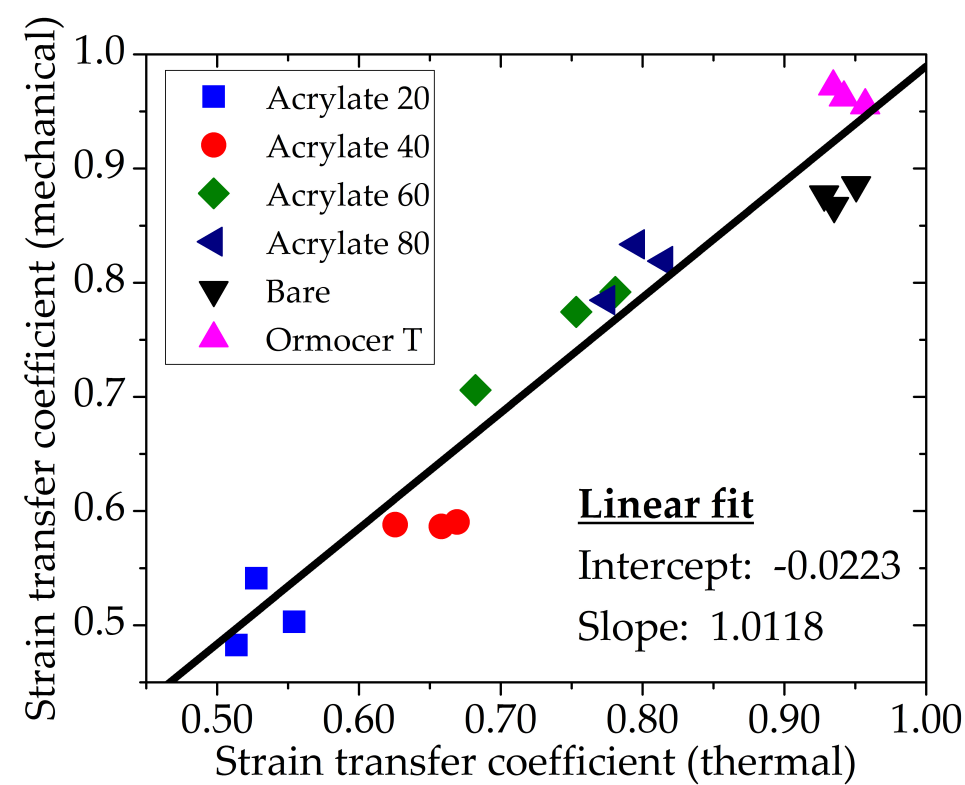

Figure 9.17: Coefficient of strain transfer derived via a strain (mechanical) versus a hydrogen measurement. Also displayed a linear fit.

coefficient of strain transfer. In addition to structural monitoring, this calibration technique could be applied to other fiber optic strain based sensors.

\subsection{Summary and conclusion}

In this chapter three different methods to determine the coefficient of strain transfer are described: Via a hydrogen, via temperature, or via strain measurement. The first two were also used for the hydrogen sensitivity calibration of the sensor.

First, the coefficient was determined via a hydrogen measurement and used for calibration. Using this coefficient for hydrogen sensitivity calibration, allowed good relative accuracy of the sensors. The average relative accuracy is better than $22 \%$ for hydrogen concentrations below $1 \%$ and better than $1 \%$ for concentrations of $1 \%$ and above. This implies that a single point hydrogen measurement can be used for the calibration of the sensor. Furthermore, the positive deviation of the accuracy at concentrations below $1 \%$ hydrogen indicates that there might be room for improvement of our model. However, more experiments have to be conducted to prove the repeatability of our results, before a change of the model is sensible.

Second, the coefficient was determined via a temperature measurement. The coefficient determined via a temperature measurement and the coefficient 
determined via a hydrogen measurement have a direct linear correlation. This implies that the coefficients can be used interchangeably and a temperature measurement can be used for a hydrogen calibration of the sensors. The average relative accuracy (of the temperature calibrated sensor) is similar to when the coefficient was determined via a hydrogen measurement, however, the relative accuracy of the individual sensors diverges more strongly. Using a temperature measurement for the hydrogen calibration has economic advantages. The temperature calibration is faster and a simple setup can be used. Furthermore, the temperature calibration has to be performed anyway, to allow for temperature compensation (see Section 3.3.2). Therefore, this method is a new and useful method for hydrogen calibration, if the lower accuracy is acceptable for the application.

Third, the coefficient was determined via a strain measurement. Here, the coefficient was not determined for a palladium based hydrogen sensor, but for an FBG surface bonded to a metal structure. The coefficient determined via a strain measurement was compared to the coefficient via a temperature measurement. Again, a direct linear correlation was found. This implies, that the coefficients can be used interchangeably and a temperature measurement can be used for a strain calibration of a surface bonded FBG. This method is unlikely to be useful for the hydrogen calibration of the sensor, due to the complex setup and constraints with the strain measurement with foil gauges. However, it might prove to be an a new and excellent method for strain calibration of surface bonded FBGs for structural health monitoring. This would allow absolute displacement measurements and tracking of the health of an individual sensor. 


\section{Chapter 10}

\section{Summary and future work}

The motivation of this thesis was to develop a fiber optic hydrogen sensor for the health monitoring of transformers. Palladium, which expands with hydrogen absorption, and FBGs, which measure this expansion, were chosen as the basis of the sensor principle. In this thesis the focus lay on increasing the hydrogen sensitivity of the sensor. This was achieved by developing a theoretical model of the response depending on the palladium geometry, new sensor designs and manufacturing methods.

The theoretical model led to useful diagrams for selection of the working temperature to avoid the phase change region and for selection of the palladium geometry to achieve a required sensitivity. This theoretical examination also showed that FBG sensors with a palladium coating would not reach the required sensitivity. As a consequence new sensor designs with large crosssectional area palladium foils were developed. For the manufacturing of these sensor special tools were designed, new processes were developed and conventional processes were adapted. Vacuum bagging proved to be an excellent method for the manufacturing of different palladium foil sensors. It also showed potential for scaling the manufacturing process since 15 sensors could be manufactured simultaneously. Furthermore, to further increase the sensitivity, a sensor design using palladium-silver foil and a new concept using a pre-strained palladium foil and FBG were developed and tested. The palladium-silver foil sensor is the most sensitive hydrogen sensor (based on palladium and FBG) reported in the literature so far. But the response time is slow and the sensor was only successfully tested up to $1 \%$ hydrogen. At higher concentrations the enormous expansion of the palladium-silver caused a delamination of the foil from the fiber. This makes the palladium-silver foil sensor only suitable for very specific applications where the highest sensitivity is necessary and a slow response and a small hydrogen concentration range are acceptable. With the pre-strained sensor the sensitivity could be significantly 
amplified. However, an ongoing relaxation process was discovered during the measurement, and has not been resolved during the course of this thesis. All sensors were tested at various hydrogen concentrations and temperatures in gas atmosphere and in oil. The ideal candidate for the implementation in a transformer proved to be a sensor based on a $100 \mu \mathrm{m}$ palladium foil. This sensor is highly sensitive, robust, and easy to manufacture in a scalable way. In addition, a new calibration method based on the coefficient of strain transfer was introduced. This coefficient could be obtained via hydrogen, temperature, and strain measurement. This enables a hydrogen calibration of the sensor via a single hydrogen measurement. Furthermore, we showed that a temperature sensitivity calibration of the hydrogen sensors can be used for the hydrogen calibration itself achieving good accuracy results. Finally, we showed that for surface bonded FBGs a temperature sensitivity calibration can be used for their strain calibration.

The sensor requirements and specifications described in Section 1.2 were met: the sensor detects dissolved and gaseous hydrogen in a range of 50-2,000 ppm in gas and oil with a resolution better $2 \mathrm{ppm} / \mathrm{pm}$ for dissolved hydrogen concentrations below $100 \mathrm{ppm}$ and a resolution better $6 \mathrm{pm} / \mathrm{ppm}$ for concentrations above (see Chapter 8). By selecting a working temperature of $90^{\circ} \mathrm{C}$ the sensor can be exposed to 16,000 ppm dissolved hydrogen before entering the phase change region (by selecting a higher working temperature the threshold can be increased, see Section 4.1.1). Furthermore, we showed that carbon monoxide only affects the response time, but does not impact the overall response and sensitivity (see Section 8.1.2). The temperature cross-sensitivity of the FBG is compensated by using a reference FBG (see Section 3.3). The temperature influence on the hydrogen solubility and thus response can be taken into account by the equations presented in Section 4.3. Additionally, the sensor proved to be reversible and no degradation of the sensors could be observed during extensive testing over 6 month in gas and oil and at temperatures up to $120^{\circ} \mathrm{C}$ (see Chapter 8).

This thesis answered many questions, but, also led to many more questions which should be answered in the future. Future work that can be derived from this work is:

- The implementation of the sensor in a real transformer will be the next important step. We are currently working on the integration following a proposed design concept with local heating of the sensor (New Zealand patent application number 734975). It is currently being tested in the laboratory and a implementation in a test transformer is planned for 2018. 
- The optimization of the response time of the foil sensor is of interest for certain applications. In this thesis, the effect of the palladium geometry on the hydrogen sensitivity was investigated in detail. But the palladium geometry also affects the diffusion time of hydrogen and thus the response time of the sensor. A investigation of foil sensors with various foil thickness would give further insight into the response time of the sensor and would be useful for future sensor development.

- Further evaluation of the palladium-silver foil sensor and testing of other palladium alloy foils is of interest. The palladium-silver foil sensor failed during a 5\% hydrogen step. It would be interesting to characterise the sensor further at low concentration, to determine its detection limits and its unloading behaviour. Furthermore, palladium-silver alloy with different silver concentrations and palladium with other alloying materials might increase the sensitivity, or suppress the phase change and should be investigated further. Since the variety of commercially available palladium alloy foils is limited, this could require the manufacturing of foils itself.

- The slow response time of the palladium-silver foil sensor may be resolved by sputter coating a thin layer of pure palladium on the foil surface. This would increase the amount of palladium atoms on the surface and thus, more hydrogen dissociation sites may be available.

- The pre-strained sensor design needs to be improved. Our results can be seen as a proof of principle, however, to increase the usefulness for an application of the sensor several challenges need to be addressed. The observed relaxation process, possibly due to creep in the adhesive interface, needs to be resolved. Furthermore, the complexity of the design should be reduced to lower the cost. Finally, the influence of vibration on the sensor and the long term stability needs to be investigated.

- The vacuum bagging method proved to be an excellent method to attach thin metal foils to optical fiber. This led to a collaboration with the team of Professor Luc Thévenaz from EPFL, Switzerland who specializes in fully distributed fiber optic sensing based on Brillouin scatter and Rayleigh backscatter. We discussed the possibility of combining our foil sensor with their distributed sensing technology. This led to the development of a sensor consisting of a 2 meter long Ormocer coated fiber with four $50 \mathrm{~mm}$ long foil segments (sandwiched $20 \mu \mathrm{m}$ palladium foil). The sensor was designed and manufactured using the vacuum bagging 
method. This sensor was sent to EPFL and is currently being evaluated using Rayleigh backscattering. These results will give an insight in the possibility of fully distributed hydrogen sensing.

- We showed that the strain transfer coefficient can be used for the strain calibration of an FBG. Furthermore, we showed that the coefficient determined via strain measurement can be replaced by the coefficient determined via a temperature measurement. The aim is to use the coefficient determined via temperature measurement (with local heating or intra day temperature fluctuation) on large civil structures to calibrate installed FBGs for the strain sensitivity and track the health of an individual sensor over time. This concept may be verified by fitting a civil structure with FBGs and reference temperature sensors, or, if available, by analysing existing data of a structure already fitted with FBGs. 


\section{Bibliography}

[1] ABB. CoreSense hydrogen and moisture sensor (2017, brochure). https: / / library.e.abb.com / public / 8d5214134354475ebbf69dc0b13b4f44 / 1LAB000585-CoreSense \%20Hydrogen\%20and\%20Moisture\%20SensorEN_Feb\%202017.pdf, viewed 25.08.2017.

[2] ABB. Benefits of transformer online dissolved gas monitoring (2016, brochure). https: / / search- ext. abb.com / library / Download. aspx? DocumentID $=1 \mathrm{LAB000601 \& LanguageCode}=$ en\&DocumentPartId $=$ \&Action=Launch, viewed 26.08.2017.

[3] AdAms, B. D., AND Chen, A. The role of palladium in a hydrogen economy. Materials today 14, 6 (2011), 282-289.

[4] Al-Mufachi, N., Rees, N., and Steinberger-Wilkens, R. Hydrogen selective membranes: a review of palladium-based dense metal membranes. Renewable and Sustainable Energy Reviews 47 (2015), 540-551.

[5] Albers, P., Pietsch, J., AND PARKer, S. F. Poisoning and deactivation of palladium catalysts. Journal of Molecular Catalysis A: Chemical 173, 1 (2001), 275-286.

[6] Alefeld, G., AND VÖLKL, J. Hydrogen in metals I - basic properties, vol. 28. Springer, Berlin, 1978.

[7] ALefeld, G., AND VÖLKL, J. Hydrogen in metals II - application-oriented properties, vol. 29. Springer, Berlin, 1978.

[8] Aleixandre, M., Corredera, P., Hernanz, M., Sayago, I., HorRILLO, M., AND GutierReZ-MonReAL, J. Study of a palladium coated Bragg grating sensor to detect and measure low hydrogen concentrations. Spanish Conference on Electron Devices (2007), 223-225.

[9] Amandusson, H., Ekedahl, L.-G., And Dannetun, H. Hydrogen permeation through surface modified $\mathrm{Pd}$ and $\mathrm{PdAg}$ membranes. Journal of Membrane Science 193, 1 (2001), 35-47. 
[10] Amano, M., Nishimura, C., AND KomaKi, M. Effects of high concentration COand $\mathrm{CO} 2$ on hydrogen permeation through the palladium membrane. Materials Transactions, JIM 31, 5 (1990), 404-408.

[11] Antunes, P., Lima, H., VArum, H., And André, P. Optical fiber sensors for static and dynamic health monitoring of civil engineering infrastructures: Abode wall case study. Measurement 45, 7 (2012), 16951705.

[12] ASTM E8M. Standard test methods of tension testing of metallic materials. ASTM International (2003).

[13] Auer, W., AND GrabKe, H. The kinetics of hydrogen absorption in palladium ( $\alpha$-and $\beta$-phase) and palladium-silver-alloys. Berichte der Bunsengesellschaft für physikalische Chemie 78, 1 (1974), 58-67.

[14] Avila, J., Matelon, R., Trabol, R., Favre, M., Lederman, D., VOLKMANN, U., AND CABRERA, A. Optical properties of Pd thin films exposed to hydrogen studied by transmittance and reflectance spectroscopy. Journal of Applied Physics 107, 2 (2010), 023504.

[15] Bal, H. K., Brodzeli, Z., Dragomir, N. M., Collins, S. F., AND SIDIROGLOU, F. Uniformly thinned optical fibers produced via HF etching with spectral and microscopic verification. Applied optics 51, 13 (2012), 2282-2287.

[16] BARAnOwski, B., MAjChrzaK, S., And Flanagan, T. The volume increase of fcc metals and alloys due to interstitial hydrogen over a wide range of hydrogen contents. Journal of Physics F: Metal Physics 1, 3 (1971), 258-261.

[17] BeUtL, M., AND LESNIK, J. Adsorption dynamics of hydrogen and carbon monoxide on V/Pd (111) alloy surfaces. Surface science 482 (2001), 353-358.

[18] Bevenot, X., Trouillet, A., Veillas, C., Gagnaire, H., And CLEMENT, M. Hydrogen leak detection using an optical fibre sensor for aerospace applications. Sensors and Actuators B: Chemical 67, 1 (2000), $57-67$.

[19] Bevenot, X., Trouillet, A., Veillas, C., Gagnaire, H., And CLEMENT, M. Surface plasmon resonance hydrogen sensor using an optical fibre. Measurement Science and Technology 13, 1 (2002), 118-124. 
[20] Birnbaum, H., AND WERT, C. Diffusion of hydrogen in metals. Berichte der Bunsengesellschaft für physikalische Chemie 76, 8 (1972), 806-816.

[21] Black, R. J., Costa, J. M., Moslehi, B., Zarnescu, L., Hackney, D., AND PETERS, K. Fiber optic temperature profiling for thermal protection heat shields. Smart Sensor Phenomena, Technology, Networks, and Systems Integration 20149062 (2014), 906204-1-12.

[22] Bodendorfer, T., Muller, M. S., Hirth, F., And Koch, A. W. Comparison of different peak detection algorithms with regards to spectrometic fiber Bragg grating interrogation systems. International Symposium on Optomechatronic Technologies (2009), 122-126.

[23] Bohmholdt, G., AND WICKE, E. Zur Diffusion von Wasserstoff und Deuterium in Palladium und Pd-Legierungen. Zeitschrift für Physikalische Chemie 56, 3_4 (1967), 133-154.

[24] Brodowsky, H. Das System Palladium/Wasserstoff. Zeitschrift für Physikalische Chemie 44, 3_4 (1965), 129-142.

[25] Brodowsky, H., And POeschel, E. Wasserstoff in Palladium/SilberLegierungen. Zeitschrift für Physikalische Chemie 44, 3_4 (1965), 143-159.

[26] Buric, M., Chen, K. P., Bhattarai, M., Swinehart, P. R., And MAKLAD, M. Active fiber Bragg grating hydrogen sensors for alltemperature operation. IEEE Photonics Technology Letters 19, 5 (2007), 255-257.

[27] BUtler, M., AND GINlEy, D. Hydrogen sensing with palladium-coated optical fibers. Journal of applied physics 64, 7 (1988), 3706-3712.

[28] Butler, M., Sanchez, R., And Dulleck, G. Fiber optic hydrogen sensor. Tech. rep., Sandia National Labs., Albuquerque, NM (United States), 1996.

[29] Butler, M. A. Optical fiber hydrogen sensor. Applied Physics Letters 45, 10 (1984), 1007-1009.

[30] Butler, M. A. Micromirror optical-fiber hydrogen sensor. Sensors and actuators B: Chemical 22, 2 (1994), 155-163.

[31] Cargol, T. An overview of online oil monitoring technologies. Fourth Annual Weidmann-ACTI Technical Conference (2005), 1-6. 
[32] Caucheteur, C., Debliquy, M., Lahem, D., And Mégret, P. Catalytic fiber Bragg grating sensor for hydrogen leak detection in air. IEEE Photonics Technology Letters 20, 2 (2008), 96-98.

[33] Chen, F., KinARI, Y., SAKamoto, F., NAKAyAma, Y., AND SAKAMOTO, Y. Hydrogen permeation through palladium-based alloy membranes in mixtures of $10 \%$ methane and ethylene in the hydrogen. International journal of hydrogen energy 21, 7 (1996), 555-561.

[34] Chowdhury, S. A., Correia, R., Francis, D., Brooks, S. J., Jones, B. J., Thompson, A. W., Hodgkinson, J., And Tatam, R. P. An optical fiber hydrogen sensor using a palladium-coated ball lens. Journal of Lightwave Technology 33, 12 (2015), 2535-2542.

[35] CHRISTMANN, K. Interaction of hydrogen with solid surfaces. Surface Science Reports 9, 1-3 (1988), 1-163.

[36] Coelho, L., De AlmeidA, J., SAntos, J., AND ViegAs, D. Fiber optic hydrogen sensor based on an etched Bragg grating coated with palladium. Applied optics 54, 35 (2015), 10342-10348.

[37] Coelho, L., Silva, S., Tafulo, P. A., Santos, J., FrazÃo, O., AND MALCATA, F. Optical fibre hydrogen sensors based on palladium coatings. International Conference on Applications of Optics and Photonics (2011), 80013Z-80013Z.

[38] Conrad, H., ERTL, G., KoCH, J., AND LatTA, E. Adsorption of CO on Pd single crystal surfaces. Surface Science 43, 2 (1974), 462-480.

[39] CZERWińsKI, A., ZAMPONI, S., AND MARASSI, R. The influence of carbon monoxide on hydrogen absorption by thin films of palladium. Journal of electroanalytical chemistry and interfacial electrochemistry 304, 1-2 (1991), 233-239.

[40] DAI, J., YANG, M., Chen, Y., CAO, K., LiAO, H., AND ZHANG, P. Sidepolished fiber Bragg grating hydrogen sensor with WO3-Pd composite film as sensing materials. Optics express 19, 7 (2011), 6141-6148.

[41] DAI, J., YANG, M., YANG, Z., LI, Z., WANG, Y., WANG, G., ZHANG, Y., AND ZHUANG, Z. Enhanced sensitivity of fiber Bragg grating hydrogen sensor using flexible substrate. Sensors and Actuators B: Chemical 196 (2014), 604-609. 
[42] DAI, J., YANG, M., YANG, Z., LI, Z., WANG, Y., ZHANG, Y., AND ZHUANG, Z. Hydrogen performance of fiber Bragg grating hydrogen sensors using Pt-loaded WO3 coating as sensing materials. Asia Pacific Optical Sensors Conference (2013), 892433-892433.

[43] DAI, J., YANG, M., YU, X., CAO, K., AND LiAO, J. Greatly etched fiber Bragg grating hydrogen sensor with $\mathrm{Pd} / \mathrm{Ni}$ composite film as sensing material. Sensors and Actuators B: Chemical 174 (2012), 253-257.

[44] DAI, J., YANG, M., YU, X., AND LU, H. Optical hydrogen sensor based on etched fiber Bragg grating sputtered with $\mathrm{Pd} / \mathrm{Ag}$ composite film. $\mathrm{Op}$ tical Fiber Technology 19, 1 (2013), 26-30.

[45] Dai, J., ZhU, L., WANG, G., XIANG, F., QIN, Y., WANG, M., AND YANG, M. Optical fiber grating hydrogen sensors: A review. Sensors 17,3 (2017), 577.

[46] Duval, M. Dissolved gas analysis: It can save your transformer. IEEE Electrical Insulation Magazine 5, 6 (1989), 22-27.

[47] Duval, M. A review of faults detectable by gas-in-oil analysis in transformers. IEEE electrical Insulation magazine 18, 3 (2002), 8-17.

[48] Duval, M. New techniques for dissolved gas-in-oil analysis. IEEE Electrical Insulation Magazine 19, 2 (2003), 6-15.

[49] Duval, M. Calculation of dga limit values and sampling intervals in transformers in service. IEEE Electrical Insulation Magazine 24, 5 (2008), 7-13.

[50] Duval, M. Smart grid monitoring of transformers by dga (2013, conference presentation). http: / / www.cigre-thailand.org/tncf / events / jwg_a2_d1_47/smart_grid_monitoring_by_duval.pdf, viewed 25.08.2017.

[51] ERIKSSON, M., AND EKEDAHL, L.-G. The influence of CO on the response of hydrogen sensitive Pd-MOS devices. Sensors and Actuators B: Chemical 42, 3 (1997), 217-223.

[52] Everett, D. Manual of symbols and terminology for physicochemical quantities and units, appendix ii: Definitions, terminology and symbols in colloid and surface chemistry. Pure and Applied Chemistry 31, 4 (1972), 577-638. 
[53] GE GRID Solutions. Hydran 201Ti (2015, brochure). https:/ / www.gegridsolutions.com / products / brochures / MD / Hydran_201Ti_ GEA12933_A4.pdf, viewed 25.08.2017.

[54] GE GRID SOlutions. Hydran M2 (2015, brochure). https: / / www.gegridsolutions.com / products / brochures / MD / HydranM2 GEA12934A_A4.pdf, viewed 25.08.2017.

[55] GE GRID SOlutions. Kelman minitrans (2015, brochure). https: / / www. gegridsolutions. com / products / brochures / MD / Minitrans_ GEA17281C_A4.pdf, viewed 25.08.2017.

[56] GeILES, T. J., AND ISLAM, S. Impact of PEV charging and rooftop PV penetration on distribution transformer life. Power and Energy Society General Meeting (2013), 1-5.

[57] GeORGILAKIS, P. S. Spotlight on modern transformer design. Springer, Berlin, 2009.

[58] Gielens, F., Knibbeler, R., Duysinx, P., Tong, H., Vorstman, M., AND KEURENTJES, J. Influence of steam and carbon dioxide on the hydrogen flux through thin Pd/Ag and Pd membranes. Journal of Membrane Science 279, 1 (2006), 176-185.

[59] GoOdFELlow. Metal - mechanical properties (website). https : / / www . goodfellowusa . com / catalog / GFUS2C . php ? ewd_token $=$ o8QKoXzjH3GJMFWOfdQwI8ntnldRgN\&n = qr8Bo85WdDqZx2HFKbZWPgcPx64ZqY\&ewd_urlNo=GFUS24\&type = 00\&prop=3, viewed 18.10.2017.

[60] Graham, T. Xxxvi.-on the relation of hydrogen to palladium. Journal of the Chemical Society 22 (1869), 419-432.

[61] Harada, S., KASAhARA, T., AND TAMAKI, S. Elastic constants and thermal expansion coefficient of hydrogenated Pd alloys. Journal of the Physical Society of Japan 54, 1 (1985), 168-174.

[62] Haran, F. M., Rew, J. K., And Foote, P. D. A strain-isolated fibre Bragg grating sensor for temperature compensation of fibre Bragg grating strain sensors. Measurement Science and Technology 9, 8 (1998), 11631166.

[63] HARLOW, J. H. Electric power transformer engineering. CRC press, 2004. 
[64] Hill, K. O., AND Meltz, G. Fiber Bragg grating technology fundamentals and overview. Journal of lightwave technology 15, 8 (1997), 1263-1276.

[65] HÜBert, T., BoON-Brett, L., BlaCK, G., ANd BANACH, U. Hydrogen sensors-a review. Sensors and Actuators B: Chemical 157, 2 (2011), 329-352.

[66] IEC 60599:2015. Minneral oil-filled electrical equipment in service Guidance on the interpretation of dissolved and free gases analysis. International Electrotechnical Commission (2015).

[67] IEC60567:1992. Guide for the sampling of gases and of oil from oilfilled electrical equipment and for the analysis of free and dissolved gases. International Electrotechnical Commission 1992 (2015).

[68] IEEE C57.104-2008. IEEE Guide for the Interpretation of Gases Generated in Oil-Immersed Transformers. Institute of Electrical and Electronics Enginers (2015).

[69] JAYARAMAN, R. Thin film hydrogen sensors: a materials processing approach, 2002.

[70] JiAnG, J., MA, G.-M., LI, C.-R., SONG, H.-T., LUO, Y.-T., AND WANG, H.-B. Highly sensitive dissolved hydrogen sensor based on sidepolished fiber Bragg grating. IEEE Photonics Technology Letters 27, 13 (2015), 1453-1456.

[71] JiAnG, J., SONG, H.-T., MA, G.-M., LI, C., LUO, Y.-T., AND WANG, H.B. Dissolved hydrogen detection in power transformer based on etched fiber Bragg grating. Instrumentation and Measurement Technology Conference (2015), 422-427.

[72] Johnson, D. Novel optical fibers-draw-tower process creates highquality FBG arrays. Laser Focus World 48, 10 (2012), 53-57.

[73] Jung, J., NAm, H., LeE, B., Byun, J. O., And Kim, N. S. Fiber Bragg grating temperature sensor with controllable sensitivity. Applied optics 38, 13 (1999), 2752-2754.

[74] Kabashima, S., OzAKI, T., AND TAKedA, N. Structural health monitoring using fbg sensor in space environment. Smart Structures and Materials 2001: Industrial and Commercial Applications of Smart Structures Technologies 4332 (2001), 78-88. 
[75] Karanja, J. M., Dai, Y., Zhou, X., LiU, B., And Yang, M. Microstructured femtosecond laser assisted FBG hydrogen sensor. Optics express 23, 24 (2015), 31034-31042.

[76] Kiskinova, M., AND BlizNAKOV, G. Adsorption and coadsorption of carbon monoxide and hydrogen on Pd (111). Surface Science 123, 1 (1982), 61-76.

[77] Kuijers, F., AND PONEC, V. The surface composition of the nickelcopper alloy system as determined by Auger electron spectroscopy. Surface Science 68 (1977), 294-304.

[78] LACHER, J. R. A theoretical formula for the solubility of hydrogen in palladium. Proceedings of the Royal Society of London. Series A, Mathematical and Physical Sciences (1937), 525-545.

[79] LatkA, I., EckeA, W., HÖFerA, B., Chojetzkia, C., And ReutLINGERB, A. Fiber optic sensors for the monitoring of cryogenic spacecraft tank structures. Proc. SPIE 5579, Photonics North 2004: Photonic Applications in Telecommunications, Sensors, Software, and Lasers (2004), 195205.

[80] LEE, S.-M., AND SIRKIS, J.-S. Hydrogen sensor based on palladiumattached fiber Bragg grating. Journal of the Optical Society of Korea 3, 2 (1999), 69-73.

[81] Lelekakis, N., Martin, D., Guo, W., And Wijaya, J. Comparison of dissolved gas-in-oil analysis methods using a dissolved gas-in-oil standard. IEEE Electrical Insulation Magazine 27, 5 (2011), 29-35.

[82] LEWIS, F. A. The palladium hydrogen system. Academic Press, London, 1967.

[83] LI, K. Review of the strain modulation methods used in fiber Bragg grating sensors. Journal of Sensors 2016 (2016), 1284520.

[84] LI, K., ZHOU, Z., AND LIU, A. A high sensitive fiber Bragg grating cryogenic temperature sensor. Chinese Optics Letters 7, 2 (2009), 121-123.

[85] LI, Y., AND CHENG, Y.-T. Hydrogen diffusion and solubility in palladium thin films. International journal of hydrogen energy 21, 4 (1996), 281-291. 
[86] LiU, Y., BAdcock, R. A., FAnG, X., FAnG, J., YAN, X., And Zhou, W. Selecting of fbg coatings for quench detection in hts coils. IEEE Transactions on Applied Superconductivity 28, 4 (2018), 1-5.

[87] Luna-Moreno, D., And Monzón-Hernández, D. Effect of the Pd$\mathrm{Au}$ thin film thickness uniformity on the performance of an optical fiber hydrogen sensor. Applied Surface Science 253, 21 (2007), 8615-8619.

[88] Luna-Moreno, D., Monzon-Hernandez, D., Calixto-Carrera, S., AND EsPINOSA-LunA, R. Tailored Pd-Au layer produced by conventional evaporation process for hydrogen sensing. Optics and Lasers in Engineering 49, 6 (2011), 693-697.

[89] Luna-Moreno, D., Monzón-Hernández, D., Villatoro, J., AND BADENES, G. Optical fiber hydrogen sensor based on core diameter mismatch and annealed Pd-Au thin films. Sensors and Actuators B: Chemical 125, 1 (2007), 66-71.

[90] LuO, Y.-T., WANG, H.-B., MA, G.-M., SonG, H.-T., Li, C., AND JiAnG, $\mathrm{J}$. Research on high sensitive D-shaped FBG hydrogen sensors in power transformer oil. Sensors 16, 10 (2016), 1641.

[91] Ma, G., Jiang, J., Li, C., Song, H., Luo, Y., And WAng, H. Pd/Ag coated fiber Bragg grating sensor for hydrogen monitoring in power transformers. Review of Scientific Instruments 86, 4 (2015), 045003.

[92] MA, G.-M., LI, C.-R., LUO, Y.-T., MU, R.-D., AND WANG, L. High sensitive and reliable fiber Bragg grating hydrogen sensor for fault detection of power transformer. Sensors and Actuators B: Chemical 169 (2012), 195198.

[93] MA, G.-M., Li, C.-R., Mu, R.-D., Jiang, J., And LuO, Y.-T. Fiber Bragg grating sensor for hydrogen detection in power transformers. IEEE Transactions on Dielectrics and Electrical Insulation 21, 1 (2014), 380385.

[94] Maier, R., Jones, B., Barton, J., McCulloch, S., Allsop, T., JONES, J., AND BENNION, I. Fibre optics in palladium-based hydrogen sensing. Journal of Optics A: Pure and Applied Optics 9, 6 (2007), 45-59.

[95] Maier, R. R., BARton, J. S., Jones, J. D., McCulloch, S., Jones, B. J., AND BURNELL, G. Palladium-based hydrogen sensing for monitoring of ageing materials. Measurement Science and Technology 17, 5 (2006), 1118-1123. 
[96] Majumder, M., Gangopadhyay, T. K., Chakraborty, A. K., DasGUPTA, K., AND BHATTACHARYA, D. K. Fibre bragg gratings in structural health monitoring- present status and applications. Sensors and Actuators A: Physical 147, 1 (2008), 150-164.

[97] MaK, T., WesterwaAl, R., Slaman, M., Schreuders, H., VAN Vugt, A., Victoria, M., Boelsma, C., And DAM, B. Optical fiber sensor for the continuous monitoring of hydrogen in oil. Sensors and Actuators B: Chemical 190 (2014), 982-989.

[98] Manchester, F., SAn-Martin, A., And Pitre, J. The H-Pd (hydrogen-palladium) system. Journal of phase equilibria 15, 1 (1994), 6283.

[99] Marchi, G., Jost, M., Steinkopff, A., Prein, C., Aszodi, A., Clausen-Schaumann, H., And Roths, J. All optical indentation probe for endoscopic diagnosis of ostheoarthritis. Optical Methods for Inspection, Characterization, and Imaging of Biomaterials II 9529 (2015), 95291A1-9.

[100] Martin, D., Lelekakis, N., Guo, W., And OdARenko, Y. Further studies of a vegetable-oil-filled power transformer. IEEE Electrical Insulation Magazine 27, 5 (2011), 6-13.

[101] Morgan Schaffer. Calisto online dissolved gas monitors (brochure). http : / / americanenergyproducts . com / documentos / Calisto \% 20 $\%$ 20Brochure \% 20and \% 20Technical \% 20Specifications . pdf, viewed 25.08.2017.

[102] Moser, H., DAhinden, V., AND BReCHNA, H. Transformerboard II: Properties and application of transformerboard of different fibres. Zurich, 1999.

[103] Nagata, H., Miyamoto, N., SAito, T., And KaizU, R. Reliable jacket stripping of optical fibers. Journal of lightwave technology 12, 5 (1994), 727729.

[104] Negri, L., Nied, A., Kalinowski, H., And Paterno, A. Benchmark for peak detection algorithms in fiber Bragg grating interrogation and a new neural network for its performance improvement. Sensors 11, 4 (2011), 3466-3482.

[105] Nguyen, T. H., Mori, S., AND Suzuki, M. Hydrogen permeance and the effect of $\mathrm{H} 2 \mathrm{O}$ and $\mathrm{CO}$ on the permeability of Pd $0.75 \mathrm{Ag} 0.25$ 
membranes under gas-driven permeation and plasma-driven permeation. Chemical Engineering Journal 155, 1 (2009), 55-61.

[106] OehlmanN, K., AND MAO, L. Evaluation of distribution network transformer dissolved gas analysis (DGA) data. Transmission and Distribution Conference and Exposition (2012), 1-4.

[107] OKazaki, S., NakagaWa, H., Asakura, S., TOMiuchi, Y., Tsuji, N., MURAYAMA, H., AND WASHIYA, M. Sensing characteristics of an optical fiber sensor for hydrogen leak. Sensors and Actuators B: Chemical 93, 1 (2003), 142-147.

[108] Othonos, A., AND KaLl, K. Fiber Bragg Grating: Fundamentals and Applications in Telecommunications and Sensing (Artech House, London, 1999) p. 97. Artech House Publishers, London, 1999.

[109] PENG, T., TANG, Y., AND SIRKIS, J. Characterisation of hydrogen sensors based on palladium electroplated fiber Bragg gratings (FBG). Proc. SPIE 3670, Smart Structures and Materials 1999: Sensory Phenomena and Measurement Instrumentation for Smart Structures and Materials (1999), 4253.

[110] Perrotton, C., Javahiraly, N., Slaman, M., Dam, B., And MEYRUEIS, P. Fiber optic surface plasmon resonance sensor based on wavelength modulation for hydrogen sensing. Optics express 19, 106 (2011), A1175-A1183.

[111] Pfiffer, M., Cormont, P., Fargin, E., Bousquet, B., Dussauze, M., LAMBERT, S., AND NÉAUPORT, J. Effects of deep wet etching in $\mathrm{HF} / \mathrm{HNO} 3$ and $\mathrm{KOH}$ solutions on the laser damage resistance and surface quality of fused silica optics at $351 \mathrm{~nm}$. Optics Express 25, 5 (2017), 4607-4620.

[112] Polking, J., Boggess, A., AND ARnold, D. Differential Equations (2nd edition). Pearson Education, London, 2006.

[113] QuAlitrol. QUALITROL DGA 150/250/400 (2012, brochure). http: / / cdn2.hubspot.net / hubfs / 227797 / Neoptix_Brochures / Iris_Powers Brochures / AP-G34-01L-01E_DGA.pdf, viewed 25.08.2017.

[114] SAAD, S., AND HASSINE, L. Hydrogen detection with FBG sensor technology for disaster prevention. Photonic Sensors 3, 3 (2013), 214-223. 
[115] Sakthivel, M., AND WePpner, W. Recent Research Developments in Chemical Engineering, Vol. 6 - Chemical sensors and technologies for sustainable hydrogen economy. Transworld Research Network, Kerala, 2005.

[116] Samsudin, M. R., Shee, Y. G., Adikan, F. R. M., Razak, B. B. A., AND DAHARI, M. Fiber Bragg gratings hydrogen sensor for monitoring the degradation of transformer oil. IEEE Sensors Journal 16, 9 (2016), 2993-2999.

[117] Schroeder, K., ECKe, W., Apitz, J., LembKe, E., AND LensChOW, G. A fibre bragg grating sensor system monitors operational load in a wind turbine rotor blade. Measurement Science and Technology 17, 5 (2006), 1167.

[118] Schroeder, K., ECKE, W., AND WILlsCH, R. Optical fiber Bragg grating hydrogen sensor based on evanescent-field interaction with palladium thin-film transducer. Optics and Lasers in Engineering 47, 10 (2009), 1018-1022.

[119] Schultz, A. M., Brown, T. D., And Ohodnicki JR, P. R. Optical and chemi-resistive sensing in extreme environments: La-Doped SrTiO3 films for hydrogen sensing at high temperatures. The Journal of Physical Chemistry C 119, 11 (2015), 6211-6220.

[120] SERVERON. Serveron TM1 (2015, brochure). http://cdn2.hubspot.net/ hubfs / 227797 / Neoptix_Brochures / Iris_Powers_Brochures / Serveron_ TM1.pdf, viewed 25.08.2017.

[121] SERVERON. Serveron TM8 (2015, brochure). http:/ / cdn2.hubspot. net / hubfs / 227797 / 2016 / Serveron_Nurture_Campaign / AP-G34-02A03E_Serveron_TM8.pdf, viewed 25.08.2017.

[122] SiEMENS. Gas-Guard 8 (2010, brochure). https: / / www.energy. siemens.com/us/pool/us/services/power-transmission-distribution/ transformer-lifecycle-management / online-dga / GasGard-FINAL.pdf, viewed 25.08.2017.

[123] SIEMENS. SITRAM H2Guard (2016, brochure). https://www. energy. siemens . com / br / pool / hq / services / power - transmission distribution / transformer-lifecycle-management/on-line-monitoring / fast - fact_SITRAM - H2Guard_monitoring - and - diagnostics_en . pdf, viewed 28.08.2017. 
[124] Silva, S., Coelho, L., Almeida, J., Frazao, O., Santos, J. L., MalCATA, F. X., BeCKer, M., Rothhardt, M., and BARTelt, H. H2 sensing based on a Pd-coated tapered-FBG fabricated by DUV femtosecond laser technique. IEEE Photonics Technology Letters 25, 4 (2013), 401-403.

[125] Silva, S. F., Coelho, L., Frazão, O., SAntos, J. L., And Malcata, F. X. A review of palladium-based fiber-optic sensors for molecular hydrogen detection. IEEE Sensors Journal 12, 1 (2012), 93-102.

[126] Simons, J., AND FlanAGAN, T. B. Absorption isotherms of hydrogen in the $\alpha$-phase of the hydrogen-palladium system. The Journal of Physical Chemistry 69, 11 (1965), 3773-3781.

[127] Sutapun, B., TABib-Azar, M., And Kazemi, A. Pd-coated elastooptic fiber optic Bragg grating sensors for multiplexed hydrogen sensing. Sensors and Actuators B: Chemical 60, 1 (1999), 27-34.

[128] Szanyi, J., KuHn, W. K., AND GoOdman, D. W. CO adsorption on $\mathrm{Pd}$ (111) and Pd (100): Low and high pressure correlations. Journal of Vacuum Science \& Technology A: Vacuum, Surfaces, and Films 11, 4 (1993), 1969-1974.

[129] Tabib-Azar, M., Sutapun, B., Petrick, R., And Kazemi, A. Highly sensitive hydrogen sensors using palladium coated fiber optics with exposed cores and evanescent field interactions. Sensors and actuators B: Chemical 56, 1 (1999), 158-163.

[130] TAM, H.-Y., LiU, S.-Y., GuAN, B.-O., ChunG, W.-H., ChAN, T. H., AND CHENG, L. Fiber bragg grating sensors for structural and railway applications. Advanced Sensor Systems and Applications II 5634 (2005), 8598.

[131] Tang, Y., Peng, Y., Sirkis, J. S., Childers, B. A., Moore, J. P., AND MELVIN, L. D. Characterization of a fiber Bragg grating (FBG) based palladium tube hydrogen sensor. Smart Structures and Materials (1999), 532-540.

[132] TIAN, X., AND CHENG, Y. The investigation of fbg sensor system for the transmission line icing measurement. International Conference on High Voltage Engineering and Application (2008), 154-157.

[133] Tien, C.-L., Chen, H.-W., LiU, W.-F., JYU, S.-S., LiN, S.-W., AND LIN, Y.-S. Hydrogen sensor based on side-polished fiber Bragg grat- 
ings coated with thin palladium film. Thin Solid Films 516, 16 (2008), 5360-5363.

[134] Touloukian, Y., Saxena, S., And Hestermans, P. Thermophysical properties of matter-the tprc data series. volume 11. viscosity. Tech. rep., THERMOPHYSICAL AND ELECTRONIC PROPERTIES INFORMATION ANALYSIS CENTER LAFAYETTE IN, 1975.

[135] Trouillet, A., Marin, E., AND Veillas, C. Fibre gratings for hydrogen sensing. Measurement Science and Technology 17, 5 (2006), 1124-1128.

[136] VAISALA. Vaisala moisture, hydrogen and temperature transmitter mht410 for online transformer condition monitoring (2016, brochure). http: / / www.vaisala.com/Vaisala\%20Documents / Brochures \%20and\% 20Datasheets / CEN - G - Power - MHT410 - Datasheet - B211484EN . pdf, viewed 25.08.2017.

[137] Villatoro, J., Diez, A., Cruz, J., And Andres, M. Highly sensitive optical hydrogen sensor using circular Pd-coated singlemode tapered fibre. Electronics Letters 37, 16 (2001), 1011-1012.

[138] Villatoro, J., Díez, A., Cruz, J. L., And Andres, M. V. In-line highly sensitive hydrogen sensor based on palladium-coated singlemode tapered fibers. IEEE sensors journal 3, 4 (2003), 533-537.

[139] Villatoro, J., Luna-Moreno, D., And Monzón-Hernández, D. Optical fiber hydrogen sensor for concentrations below the lower explosive limit. Sensors and Actuators B: Chemical 110, 1 (2005), 23-27.

[140] WAN, K. T., LeUnG, C. K., AND Olson, N. G. Investigation of the strain transfer for surface-attached optical fiber strain sensors. Smart $M a-$ terials and Structures 17, 3 (2008), 035037.

[141] WANG, M., WANG, D., YANG, M., CHENG, J., AND LI, J. In-line machzehnder interferometer and FBG with Pd film for simultaneous hydrogen and temperature detection. Sensors and Actuators B: Chemical 202 (2014), 893-896.

[142] WEIDMANN. InsuLogix H hydrogen monitor. http:/ / www.weidmanndiagnostics.com/images/InsuLogixH_2-1-2015.pdf, viewed 28.08.2017.

[143] WesterwaAl, R., Gersen, S., Ngene, P., Darmeveil, H., SchreudERS, H., MiddelKoop, J., AND DAM, B. Fiber optic hydrogen sensor 
for a continuously monitoring of the partial hydrogen pressure in the natural gas grid. Sensors and Actuators B: Chemical 199 (2014), 127-132.

[144] WICKE, V. E., AND NERNST, G. Zustandsdiagramm und thermodynamisches Verhalten der Systeme Pd/H2 und Pd/D2 bei normalen Temperaturen; H/D-Trenneffekte. Berichte der Bunsengesellschaft für physikalische Chemie 68, 3 (1964), 224-235.

[145] Willsch, R., ECKE, W., AND BARTELT, H. Optical fiber grating sensor networks and their application in electric power facilities, aerospace and geotechnical engineering. 15th Optical Fiber Sensors Conference Technical Digest (2002), 49-54.

[146] Xiang, F., Wang, G., QIN, Y., YANG, S., Zhong, X., DAI, J., AND YANG, M. Improved performance of fiber Bragg hydrogen sensors assisted by controllable optical heating system. IEEE Photonics Technology Letters (2017), 1233-1236.

[147] YABLON, A. D. Optical fiber fusion splicing, vol. 103. Springer, Berlin, 2005.

[148] YamadA, T., Onishi, T., AND TAMARU, K. Adsorption-desorption kinetics of carbon monoxide on palladium polycrystalline surfaces. Surface science 133, 2-3 (1983), 533-546.

[149] YANG, M., LiU, H., ZhanG, D., AND TONG, X. Hydrogen sensing performance comparison of $\mathrm{Pd}$ layer and $\mathrm{Pd} / \mathrm{WO} 3$ composite thin film coated on side-polished single-and multimode fibers. Sensors and Actuators B: Chemical 149, 1 (2010), 161-164.

[150] YANG, M., WANG, G., DAI, J., YANG, Z., LI, Z., WANG, Y., ZHANG, Y., AND ZHUANG, Z. Fiber Bragg grating sensors with Pt-loaded WO3 coatings for hydrogen concentration detection down to $200 \mathrm{ppm}$. Measurement Science and Technology 25, 11 (2014), 114004.

[151] YANG, Z., ZhanG, M., LiaO, Y., TiAn, Q., LI, Q., ZHANG, Y., AND ZHUANG, Z. Extrinsic Fabry-Perot interferometric optical fiber hydrogen detection system. Applied optics 49, 15 (2010), 2736-2740.

[152] YEO, T., SUN, T., AND GRATTAN, K. Fibre-optic sensor technologies for humidity and moisture measurement. Sensors and Actuators A: Physical 144, 2 (2008), 280-295. 
[153] YU, C., LiU, L., Chen, X., LiU, Q., AND Gong, Y. Fiber-optic FabryPerot hydrogen sensor coated with Pd-Y film. Photonic Sensors 5, 2 (2015), 142-145.

[154] YU, Z., JiN, L., CHEN, L., LI, J., RAN, Y., AND GUAN, B.-O. Microfiber Bragg grating hydrogen sensors. IEEE Photonics Technology Letters 27, 24 (2015), 2575-2578.

[155] YUAN, Y., WANG, L., DING, L., AND WU, C. Theory, experiment, and application of optical fiber etching. Applied optics 51, 24 (2012), 58455849.

[156] ZalvideA, D., Diez, A., CRuz, J., AND Andres, M. Wavelength multiplexed hydrogen sensor based on palladium-coated fibre-taper and Bragg grating. Electronics Letters 40, 5 (2004), 301-302.

[157] Zhang, Y.-N., Peng, H., QIAN, X., Zhang, Y., AN, G., AND ZHAO, $Y$. Recent advancements in optical fiber hydrogen sensors. Sensors and Actuators B: Chemical (2017), 393-416.

[158] ZhOU, X., DAi, Y., Zou, M., Karanja, J. M., AND YANG, M. FBG hydrogen sensor based on spiral microstructure ablated by femtosecond laser. Sensors and Actuators B: Chemical 236 (2016), 392-398.

[159] ZHOU, Z., AND OU, J. Techniques of temperature compensation for FBG strain sensors used in long-term structural monitoring. Fundamental Problems of Optoelectronics and Microelectronics II 5851 (2005), 167-173.

[160] Zou, M., DAI, Y., ZHOU, X., DONG, K., AND YANG, M. Femtosecond laser ablated FBG with composite microstructure for hydrogen sensor application. Sensors 16, 12 (2016), 2040. 